\title{
Extracorporeal Membrane Oxygenation Support in Post-Cardiotomy Shock
}

Citation for published version (APA):

Raffa, G. M. (2019). Extracorporeal Membrane Oxygenation Support in Post-Cardiotomy Shock. [Doctoral Thesis, Maastricht University]. ProefschriftMaken. https://doi.org/10.26481/dis.20191216gr

Document status and date:

Published: 01/01/2019

DOI:

10.26481/dis.20191216gr

Document Version:

Publisher's PDF, also known as Version of record

\section{Please check the document version of this publication:}

- A submitted manuscript is the version of the article upon submission and before peer-review. There can be important differences between the submitted version and the official published version of record.

People interested in the research are advised to contact the author for the final version of the publication, or visit the DOI to the publisher's website.

- The final author version and the galley proof are versions of the publication after peer review.

- The final published version features the final layout of the paper including the volume, issue and page numbers.

Link to publication

\footnotetext{
General rights rights.

- You may freely distribute the URL identifying the publication in the public portal. please follow below link for the End User Agreement:

www.umlib.nl/taverne-license

Take down policy

If you believe that this document breaches copyright please contact us at:

repository@maastrichtuniversity.nl

providing details and we will investigate your claim.
}

Copyright and moral rights for the publications made accessible in the public portal are retained by the authors and/or other copyright owners and it is a condition of accessing publications that users recognise and abide by the legal requirements associated with these

- Users may download and print one copy of any publication from the public portal for the purpose of private study or research.

- You may not further distribute the material or use it for any profit-making activity or commercial gain

If the publication is distributed under the terms of Article $25 \mathrm{fa}$ of the Dutch Copyright Act, indicated by the "Taverne" license above, 
Extracorporeal Membrane Oxygenation Support in Post-Cardiotomy Shock

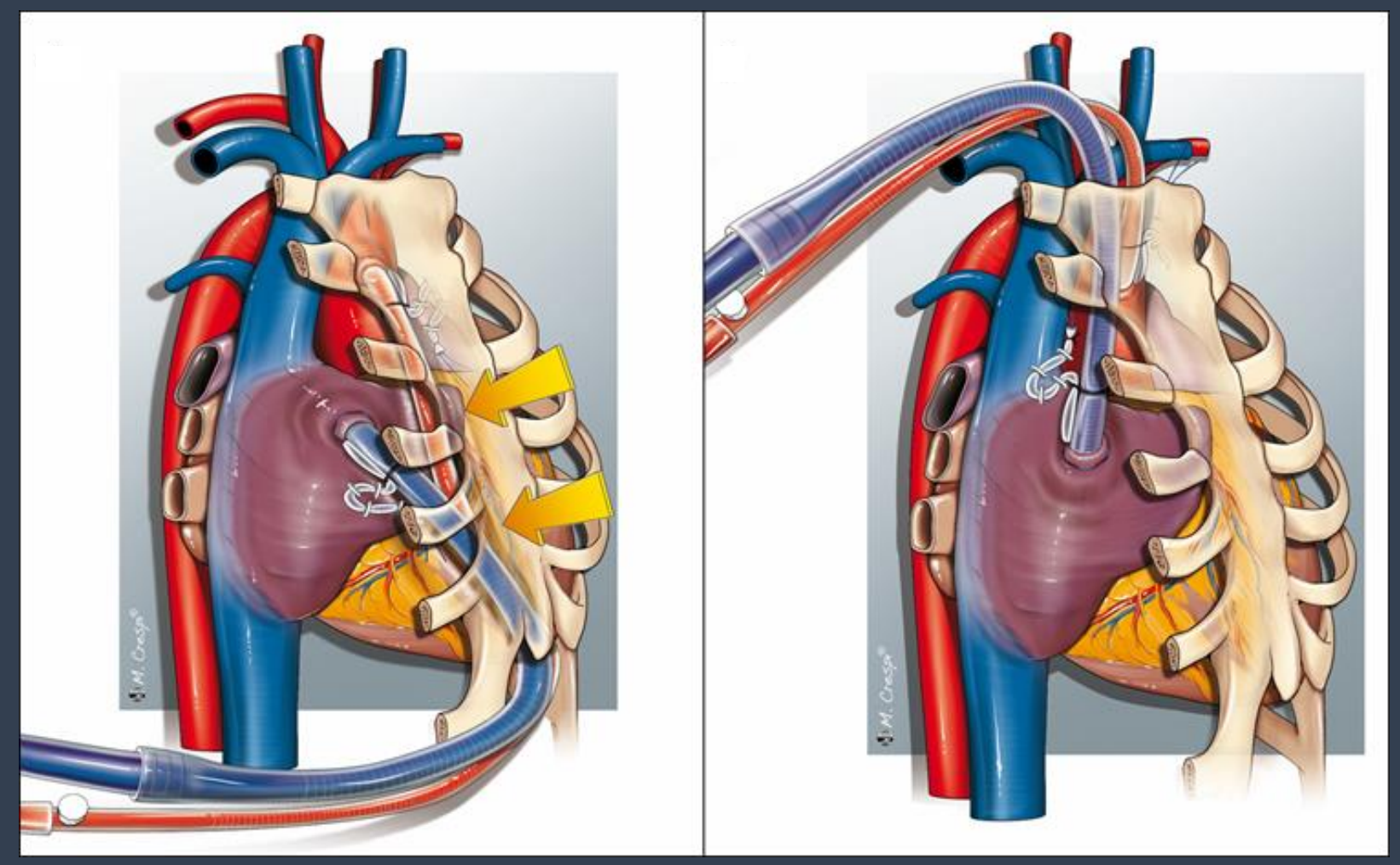

Giuseppe Maria Raffa 


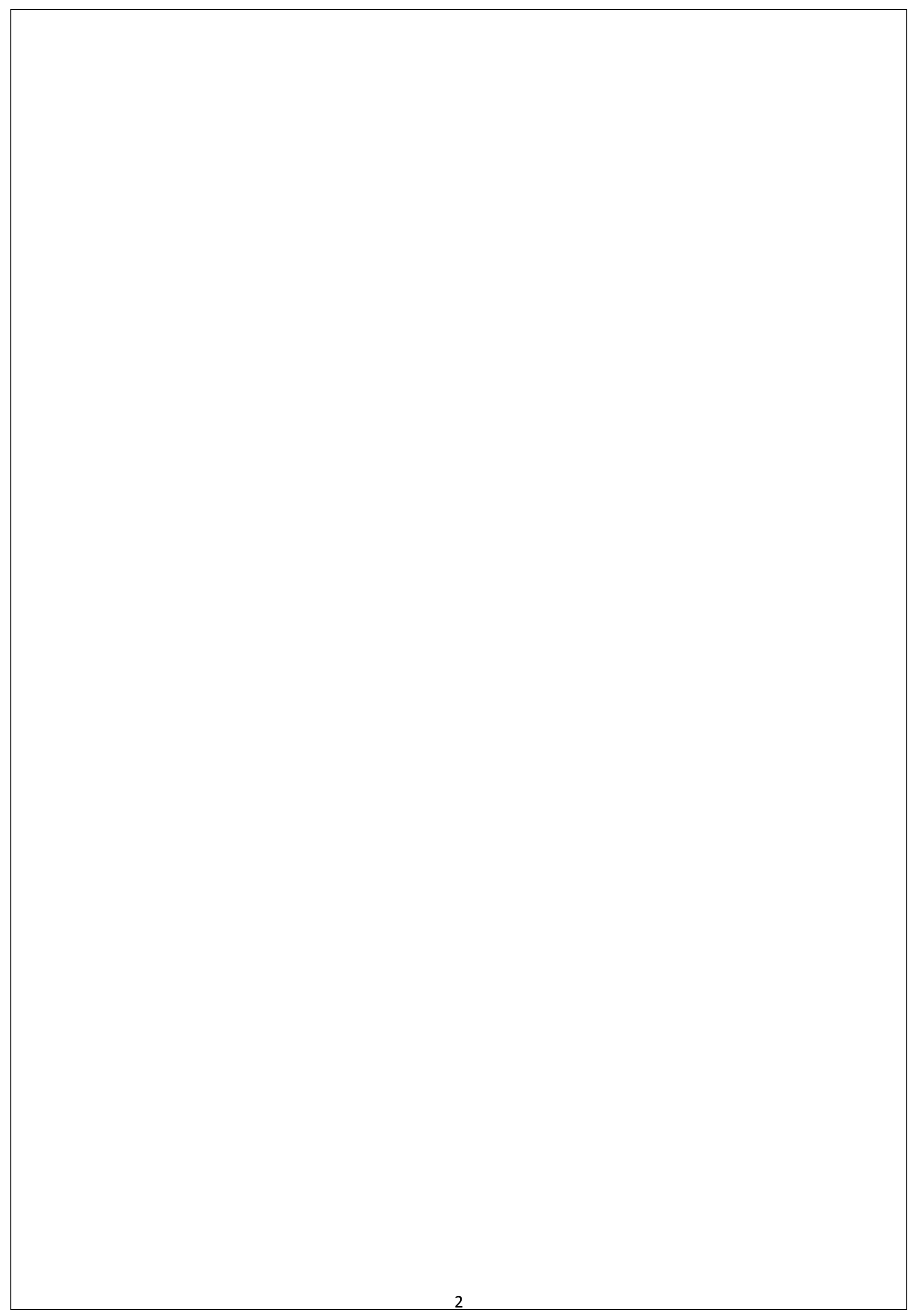




\section{Extracorporeal Membrane Oxygenation Support in Post-Cardiotomy Shock}

\section{Giuseppe Maria Raffa}

Maastricht University Medical Center 
(C) copyright Giuseppe Maria Raffa, Maastricht 2019

Printing: ProefschriftMaken || www.proefschriftmaken.nl

Lay-out: Giuseppe Maria Raffa

ISBN 978-94-6380-653-4 


\title{
Extracorporeal Membrane Oxygenation Support in Post-Cardiotomy Shock
}

\author{
DISSERTATION \\ to obtain the degree of Doctor at Maastricht University, \\ on the authority of the Rector Magnificus, Prof. Dr. Rianne M. Letschert \\ in accordance with the decision of the Board of Deans, \\ to be defended in public \\ on December 16, 2019; at 10.00 hours \\ by
}

Giuseppe Maria Raffa 


\section{Promotors}

Prof. dr. Roberto Lorusso

Prof. dr. Jos G. Maessen

\section{Co-Promotor}

Dr. Jan Willem Sels

\section{Assessment committee}

Chair: Prof. dr. Wolfgang F.F.A. Buhre

Prof. dr. Bart Meyns (Leuven, Belgium)

Prof. dr. Udo Boeken (Dusseldorf, Germany)

Prof. dr. Arnoud W.J. van t'Hoff 
Chapter 1 Introduction and Outline of the Thesis.....................9

Chapter 2 Heart Transplant Program at IRCCS-ISMETT: Impact of Mechanical Circulatory Support on Pre- and Post -Transplant Survival...............................................31

Chapter 3 In-Hospital Outcome of Post-Cardiotomy Extracorporeal Life Support in Adult Patients: the 2007-2017 Maastricht Experience.........................................45

Chapter 4 Meta-Analysis of Peripheral or Central ECMO in Postcardiotomy and Non-Postcardiotomy Shock...........63

Chapter 5 In-Hospital Outcomes after Emergency or Prophylactic VenoArterial Extracorporeal Membrane Oxygenation during Transcatheter Aortic Valve Implantation: A Comprehensive Review of the Literature 95

Chapter 6 Structured Review of Post-Cardiotomy Extracorporeal Membrane Oxygenation: Part 1 - Adult Patients............115

Chapter 7 Structured Review of Post-Cardiotomy Extracorporeal Membrane Oxygenation: Part 2 - Pediatric Patients.........155 
Chapter 8 Pulmonary Artery Cannulation to Enhance Extracorporeal Membrane Oxygenation Management in Acute Cardiac Failure 191

Chapter 9

General discussion. 209

Valorization .215

Summary 219

Samenvatting .223

Acknowledgements. .227

About the author. .231

List of publications 233 
CHAPTER 1

GENERAL INTRODUCTION 


\section{List of Abbreviations}

$\mathrm{ECMO}=$ extracorporeal membrane oxygenation

$\mathrm{V}-\mathrm{V}=$ veno-venous

$\mathrm{V}-\mathrm{A}=$ veno-arterial

$\mathrm{PC}=$ post cardiotomy

$\mathrm{VAD}=$ ventricular assist device

ELSO $=$ Extracorporeal Life Support Organization

$\mathrm{AMI}=$ acute myocardial infarction

$\mathrm{LV}=$ left ventricular

$\mathrm{LA}=$ left atrium

$\mathrm{CABG}=$ coronary artery bypass graft

Htx $=$ heart transplantation

LVAD = left ventricle assist device

$\mathrm{RV}=$ right ventricular

$\mathrm{RVAD}=$ right ventricle assist device

$\mathrm{CPB}=$ cardiopulmonary bypass

LVEDP $=$ left ventricle end diastolic pressure

IABP = intra-aortic balloon pump

$\mathrm{ICU}=$ intensive care unit

ECLS $=$ extracorporeal life support

$\mathrm{CNS}=$ central nervous system

$\mathrm{ECPR}=$ extracorporeal cardiopulmonary resuscitation

$\mathrm{CPR}=$ cardiopulmonary resuscitation

TAVI $=$ transcatheter aortic valve implantation 
ECMO is increasingly used in cases of acute cardiac and lung failure refractory to conventional treatment. ECMO may allow the dysfunctional organs to recover, or to provide a "bridge-to-decision" approach for more advanced treatment. ${ }^{1-5}$ The quick availability, ease of application, and reliability have made ECMO a more attractive option compared to other implantable or para-corporeal $\mathrm{VAD}^{6-}$ ${ }^{11}$, particularly in the PC setting. ${ }^{1-5} \mathrm{PC}$ cardiogenic shock has become the most frequent indication for ECMO in the United States $^{3}$, but, its exponential increase did not parallel improved clinical outcomes. ${ }^{5,12}$ Reports addressing PC-ECMO are limited, with only a few single-centre experiences available in the literature ${ }^{13-36}$, commonly including adult and pediatric populations in the same series, or combining the PC-ECMO cases with the institutional global ECMO experience. ${ }^{37-54}$

The prevalence of ECMO implementation in PC patients ranges between $0.4 \%$ and $3.65 \% .^{1-5}$ Maxwell and colleagues evaluated more than 9,000 ECMO patients in the Nationwide Inpatient Sample database in United States from 1998 to 2009, and 4,493 patients had PC-ECMO, representing almost $50 \%$ of the total number of cases although a relative decrease during years was observed. ${ }^{4}$ McCarthy confirmed this decline in PC-ECMO from 2002 (56.9\%) to 2011 (37.9\%) analyzing the same database. ${ }^{2}$ Nevertheless, PC-ECMO represented the most common indication for every year analyzed in this study indicating that, in absolute numbers, PC continues to represent the most frequent scenario for ECMO use. ${ }^{2}$ Recently, the data from the ELSO Registry, which includes almost 90,000 ECMO adults and children cases, have confirmed the substantial rise in PC-ECMO use, particularly during the last 2-3 years. ${ }^{5}$

Concerning the patients' characteristics, although PC-ECMO candidates are predominantly young and undergoing emergency operations ${ }^{16}$, elderly patients are increasingly considered for such a mechanical support. Age of $>65$ years has not been regarded as an absolute contraindication to V-A or V-V ECMO candidacy ${ }^{55,56}$, although long- term outcomes remain unreported. Other clinical characteristics include renal insufficiency, AMI, presence of left-main disease, LV dysfunction, 
prolonged history of coronary artery disease with previous AMI, prior open heart surgery, and urgent or emergent status. ${ }^{16} \mathrm{PC}$-ECMO is most frequently associated with CABG patients, followed by valve surgery, and combined valve/coronary surgery. Noteworthy, in several series, acute aortic dissection repair did not represent a contraindication to PC-ECMO. Primary graft failure represents the most common causes of death after native Htx, followed by RV failure, and acute rejection or sepsis. ${ }^{57}$ ECMO use post Htx has been reported as high as $10 \%-15 \% .{ }^{58-60}$ The use of more marginal donor hearts may predispose to the need for PC-ECMO. Notably, Listijono and colleagues showed that its use apparently does not influence primary graft recovery or patient survival to discharge ${ }^{61}$ After VAD implantation, RV failure may occur in up to $25 \%$ of the supported patients. ${ }^{62,63}$ PC-ECMO is often considered in this setting to support the RV while it recovers, or, to bridge to RVAD, when necessary. ${ }^{63,64}$

In a variety of ECMO series, the most common indication for PC-ECMO is the inability to wean from $\mathrm{CPB}$, followed by refractory cardiogenic shock (defined also by degree of chemical support/inotrope score) and cardiac arrest in the intensive care unit. ${ }^{13-34}$ Unless a detailed analysis of the causes or conditions which required the PC-ECMO are missing, it is evident that the use of ECMO for PC cardiac arrest has been more frequently considered during the last 10 years, with a constant increase over time ${ }^{65}$ with promising results as reflected by latest 2017 Society of Thoracic Surgeons Expert Consensus for the Resuscitation of Patients Who Arrest After Cardiac Surgery. ${ }^{66}$

Location of ECMO implantation is influenced by the indication for implant, cardio-circulatory or respiratory needs, and availability of the ECMO circuit, staff, and the patient's condition. Implantation in the operating room occurs most frequently, followed by the ICU, with rare placement on the ward. The timing of ECMO implantation after surgery is dictated by the patient's condition, e.g. during cardiac arrest vs. as cardiogenic shock-related conditions, and, occasionally, by the 
logistics of gathering the ECMO team and the ECMO system. Delayed identification of diagnosis and clinical status of the patient may influence the time of implantation. ${ }^{29}$

In spite of the presence of an already available surgical access (sternotomy or thoracotomy) for central cannulation, the peripheral approach for ECMO implant is more commonly used. The peripheral cannulation has been the only access employed in three series ${ }^{20,25,28}$, whereas a central approach was the unique access in one experience. ${ }^{27}$ Open peripheral cannulation (vs percutaneous cannula placement) was chosen in the majority of the series ${ }^{17,25,27}$ and has been associated with fewer complications than the percutaneous approach. ${ }^{25,27}$ Rastan and associates showed that femoral venous drainage was associated with worse prognosis suggesting that suboptimal right sided decompression had a negative impact on ECMO flow and management. ${ }^{21}$ In contrast, central cannulation has been associated with higher rates of bleeding and continuous V-V hemofiltration rates as compared to the peripheral access. ${ }^{43}$ Alternative approaches, e.g arterial inflow via the subclavian artery with either peripheral $^{18,19,31,67}$ or central ${ }^{68}$ cannulation for venous return, are additional configurations. The benefit of central cannulation lies in improved decompression of heart chambers and antegrade flow from the proximal aorta. It is often associated to the chest open to allow the unimpeded exit of the atrial and aortic cannulas and/or to prevent any mechanical cardiac compression, which might inhibit venous return, a situation which can last for days, particularly in the face of RV failure with slow recovery. ${ }^{21,26}$ In the case of central cannulation, atrial and aortic cannulas may be tunneled to exit at the subxyphoid region, which allows sternal closure and may allow patient extubation and mobilization after surgery in case of prolonged support, but it may impede adequate cardiac filling during weaning. In case of an expected short-term support with an expeditious recovery, jugular exit sites might be contemplated by mobilizing the sternocleidomastoid muscle insertion and extending the upper sternal incision with a Y towards the head of the patient. This approach allows the aortic and atrial cannulas to lay vertically, exiting cranially without impinging on the heart during weaning 
tests prior to re-sternotomy. The use of right anterior mini- thoracotomy for ascending aorta, right atrium and pulmonary vein (for venting) access, has also been reported. ${ }^{69,70}$

With regards to femoral access, the so-called "pseudo-percutaneous approach" may be applied, with surgical exposure of the femoral vessels, and the cannulas are tunneled through an inferior thigh incision, allowing the closure of the skin. ${ }^{70,71}$ Dilatation of the LV due to insufficient ejection either for absent LV contractility or high afterload secondary to high retrograde ECMO flow towards the aortic valve , may lead to blood stasis in the left-sided cardiac chambers with a risk of stasis and an elevated LVEDP causing pulmonary edema, hypoxia and clot formation in the LV or LA. V-A ECMO usually results in effective right-sided cardiac drainage, but may not be as effective in decompressing the left chambers. In these circumstances, the addition of catheter drainage, specifically in the left cardiac cavities, may be warranted to fully decompress the cardiac left side. Cannula placement in the pulmonary artery or the use of a concomitant IABP may be sufficient, but not in all the cases. The use of suction device or surgically placed catheter in the LA or LV may be a mandatory approach to obtain effective LV decompression ${ }^{72,73}$, although the clinical benefit of LV decompression is yet to be proven.

Another controversial issue is the benefit of concomitant use of IABP during ECMO support. It ranges from $12 \%$ to $100 \%$ of the ECMO cases, underlining the heterogeneity of the patient's disease and the lack of certainty regarding the benefits of the combination of ECMO and IABP support. ${ }^{74}$ In most instances, IABP represents the first line of support used in the operating room when facing failure to wean from $\mathrm{CPB}$ or when perioperative cardiac dysfunction appears soon after CPB weaning in the operating room or ICU. ${ }^{27}$ In ECMO, the IABP is usually used to enhance the flow pulsatility while decreasing afterload, and thus, enhancing LV ejection and aortic valve opening. This reduces left heart chamber-related blood stasis by increasing ejection while simultaneously increasing coronary perfusion. ${ }^{19,20,71,75}$ Nevertheless, in patients fully supported by PC-ECMO, IABP benefit 
may be limited, ${ }^{31,32}$ and, with peripheral PC-ECMO, the IABP may be competing for femoral arterial

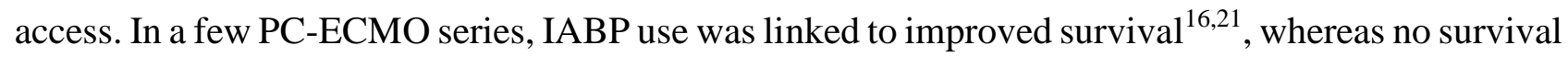
difference was found in many others. ${ }^{18,22,23,25,26,39,46}$ It deserves mention that the presence of IABP did not increase the risk of limb ischemia, as those authors uniformly using IABP in PC-ECLS showed no or very low rates of leg-related complications. ${ }^{13,20,24}$

In some cases, the need for perioperative ECMO may be predictable, due to the poor clinical conditions of the patients thereby presaging unmanageable cardiac failure post $\mathrm{CPB}$. In such a setting, a timely ECMO initiation ${ }^{77}$, even including its prophylactic use, may provide significant benefits despite the associated high risk of complications. It allows a smoother perioperative course with reduced need for vasopressors and the avoidance of severe hemodynamic instability, with further cardiac, lung and multi-organ deterioration.

The use of inotropes or vasoactive drugs remain another controversial issue in PC-ECMO. Enhanced cardiac contraction may be helpful to prevent intracardiac stasis and permit ECMO weaning ${ }^{78}$, but it may further increase the myocardial work, oxygen consumption and recovery. Recently, it was shown that pre-treatment with Levosimendan (Symdax ${ }^{\circledR}$, Abbot) seems to facilitate weaning from V-A ECMO, reducing the need for high-dose inotropes. ${ }^{79}$

ECMO duration varies a great deal among published literature for PC-ECMO series, but rarely are patients supported longer than 15 days. Several investigators advocate short (48-72 hours) support and, if insufficient recovery occurs, to implement a more advanced mechanical support, particularly if the patient is not an ideal candidate for HTx. ${ }^{16,21,26}$ The availability of a VAD may, however, not be present in all cardiac surgery centres, underlining the need for close relationships with heart failure center (with VAD and HTx programs) for all cardiac centers without such programs, to facilitate patient referrals when indicated. Longer durations on ECMO may be effective in some specific cases, 
e.g. after heart transplantation, to allow myocardial recovery or resolution of refractory pulmonary hypertension. ${ }^{15}$

Complications in ECMO patients are common and frequently determine the final outcome.${ }^{80}$ Bleeding represents the most frequent complications in PC-ECMO, occurring in up to $90 \%$ of patients in some series. ${ }^{13,19}$ It is well-known that PC-ECMO patients are at high risk for hemorrhagic events due to the combination of surgery-related bleeding magnified by the use and need of heparinization for the ECMO circuit. Many investigators advocate the avoidance of heparin infusion for the first 12-48 hours in PC-ECMO. ${ }^{17,20}$ Heparin can be held for even longer periods in the case of continued bleeding postoperatively. ${ }^{15,17,20,21,23,29,46}$ Along with bleeding, there are additional adverse events that are similar to other ECMO settings, but the rates are higher in PC-ECMO as a result of the preceding $\mathrm{CPB}$ run and the cardiac surgery operations. CNS complications in $\mathrm{V}-\mathrm{A}$ and $\mathrm{V}-\mathrm{V}$ ECMO have recently been shown to occur in $15 \%$ and $7 \%$ of adult patients, respectively. ${ }^{81,82}$ In PC-ECMO, these rates appear to be much higher, occurring in up to $30 \%$ of patients. ${ }^{13,15,16,20} \mathrm{~A}$ high level of suspicion, appropriate neuro-monitoring, and ECMO management modification ${ }^{83}$ may influence CNS complications. Renal failure and leg ischemia rates at a rate similar to other indications for ECMO. The use of ipsilateral distal perfusion cannula reduces the leg ischemia-related events. ${ }^{21}$ Delay in cannula placement, triggered by signs of ischemia, may lead to a reperfusion injury with a poor outcome $^{27}$. Femoral artery access can be achieved by cannulating a Dacron or Hemashield prosthetic graft $(6-8 \mathrm{~mm})$ anastomosed end to side onto the femoral artery thus maintaining arterial flow to the ipsilateral lower limb ${ }^{20}$. In PC-ECMO, continuous monitoring of the adequacy of limb perfusion is no different from the management applied to other ECMO settings with peripheral arterial access. ${ }^{70}$

Successful weaning from PC-ECMO varies amongst published series, ranging from $31 \%$ to $76 \%$, with almost half of the experiences showing a weaning rate at or slightly above $50 \%$, but hospital discharge ranges from $16 \%$ to $52 \%$, with less than $30 \%$ of centers reporting a survival rate above 
40\%. In fact, despite a dramatically improved technology and management experience, the PCECMO-related survival has not improved in the last 20 years. ${ }^{23}$ Based on the most recent report from the ELSO Registry database on $\mathrm{PC}-\mathrm{ECMO}^{5}$ a gradual decline during the most recent years has occurred, reaching $15 \%$ survival in the most recent era. Transition from ECMO to VAD support or HTx appears to improve in-hospital survival, as shown by Smedira and coworkers. ${ }^{16}$ They found a $75 \%$ short-term survival in patients supported by VAD after a short ECMO run. ${ }^{16}$ However, the employment of VAD placement to wean from PC-ECMO has not been reported in more than $17 \%$ of patients in any series, with VAD/HTx centers using this capability in only $4 \%-6 \%$ of ECMO supported patients.

Multi-organ system failure, despite recovery from cardiac failure, appears to play a significant role in PC-ECMO patients, occurring even after successful ECMO weaning, mostly due to sepsis and infection. ${ }^{19,20}$

The cause of death may be misleadingly interpreted in ECMO patients, as reported by Rastan and associates who showed that an unexpected cause of death was found in almost $30 \%$ of autopsies, and an unrecognized concomitant illness was determined in $80 \%$ of the patients. ${ }^{84}$ Overall, that study showed that mortality was cardiac in origin in over $60 \%$ of patients, multi-organ system failure in $10 \%$, and due to neurologic complications in $5 \% .^{84}$

The survival of patients on PC-ECMO after Htx appears favorable, allowing recovery of the graft in most patients. ${ }^{85}$ Takeda and colleagues recently compared patient outcome after primary graft failure with PC-ECMO support versus VAD implantation, and showed that PC-ECMO had significantly better outcomes, particularly with respect on post-implant re-thoracotomy for bleeding (30\% vs. $70 \%$, $\mathrm{p}<0.01)$, renal replacement therapy $(11 \%$ vs. $53 \%, \mathrm{p}<0.01)$, duration of support $(5.2 \pm 3.9$ days vs. $14 \pm 17$ days, $\mathrm{p}=0.011)$, and higher weaning rates $(89 \%$ vs. $59 \%, \mathrm{p}=0.03)$, with a trend towards better survival-to hospital-discharge (41\% vs. $19 \%) .{ }^{86}$ 
Long-term outcomes after hospital discharge appears favorable, with the vast majority of patients still alive at 1-year follow-up. Saxena and colleagues showed that in a high-risk cohort of patients over 70 years of age, survival was $69 \%$ at 3 years, and $51 \%$ at 5 years, confirming the good post-discharge prognosis of PC-ECMO patients, even if with older age. ${ }^{29}$

Patient age and survival requires a distinct discussion in the context of PC-ECMO, as many patients eligible for such support are elderly. In an analysis of patients older than 65 versus younger subjects supported by ECMO for PC refractory cardiopulmonary failure, Narotsky found unfavorable survival to discharge and at one year $(30 \%)$ in elderly patients. ${ }^{49}$ When the analysis was adjusted for confounders, however, age turned out not to be statistically significant as a predictor for in-hospital death, though a trend for increased risk was still present. A linear relationship between mortality and age has been demonstrated, but an age cut-point could not be identified. ${ }^{22}$ Satio and colleagues analyzed 91 patients submitted to emergency ECMO for a variety of reasons including PC, and found that age was not a predictor of mortality, indicating that elderly patients, although needing more time to recover, can benefit from $\mathrm{ECMO}^{3.9}$ Although a recent large database evaluation has shown that older patients undergoing ECMO implant for cardiac or respiratory indications have indeed a significantly worse prognosis, nevertheless, the data does not support older age as an absolute contraindication to $\mathrm{PC}-\mathrm{ECMO}^{87}$.

Prediction of PC-ECMO weaning and survival has been addressed by several investigators. ${ }^{13-34}$ Preimplant, intra-operative factors, as well as post ECMO events and the patient's physiologic response to circulatory support, all play a critical role in the final outcome. Among pre-ECMO factors, ECPR, or ECMO institution during a cardiac arrest, was a strong negative predictor in several experiences. ${ }^{13,15,29}$ In other series, neither CPR nor the time spent in resuscitation had significant effect on survival. ${ }^{17,31,32}$ Lactate concentration at the time of ECMO initiation or its highest level at 12 or 24 hours post $\mathrm{ECMO}^{18,21,25,28,29,34,36,42,80}$, renal and liver failure ${ }^{88-90}$, respiratory failure ${ }^{91,92}$, and 
duration of ECMO ${ }^{15,17,25,26}$ may influence the outcome. No significant survival effect of hospital location at time of ECMO institution was observed, e.g. operating room, ICU, or ward. ${ }^{26}$

The use of V-V ECMO has been increasingly employed as therapy for primary, refractory respiratory failure as a result of the outcomes seen in CESAR trial. ${ }^{93}$ There are very few reports, however, specifically addressing the use of $\mathrm{V}-\mathrm{V}$ configuration for PC-ECMO in PC patients. Nakamura and colleagues explored the outcome of V-V ECMO in 11 PC patients after various cardiac surgery procedures and with ages ranging from 35 to 83 years. ${ }^{65}$ This series has shown a favorable overall outcome (7 patients discharged, $63.6 \%$ survival), with age as the only predictor of a poor prognosis. Interestingly, Song and co- workers recently reported their experience in 13 PC patients supported with V-V PC-ECMO for ARDS, with age playing no role in predicting survival (overall in-hospital survival 69.3\%). ${ }^{66}$ In this series, 7 patients received V-V PC-ECMO within 24 hours from surgery, the remainder (6 patients) at a median of 8.5 days (range 5-16) from surgery. The RESP score ${ }^{94}$ proved to be a good predictor of survival, ( $100 \%$ in class III, $50 \%$ in class IV, and $20 \%$ in Class V). Noteworthy, in this experience, septic shock was responsible for all deaths while on ECMO.

Based on the usual rate of respiratory complications following cardiac surgery (prolonged ventilation occurs in approximately $10 \%$ of patients, cite STS report) it appears that there is a paucity of published reports on V-V ECMO therapy for this complication. ${ }^{91,92}$ Further research is needed to understand the indications for V-V PC-ECMO, e.g avoiding ventilation-induced lung injury, improving gas exchange, and decreasing systemic ischemia.

\section{Aim and outline of the thesis}

The purpose of this thesis was to analyze the in-hospital outcome (survival and complications occurrence) of ECMO in the treatment of PC shock. To achieve this goal, several investigations were 
performed, by means of original studies, meta-analysis and reviews. Two single institution experiences (ISMETT hospital in Italy and University of Maastricht Medical Centre in Netherlands) were retrospectively reviewed in order to assess the results of ECMO in PC-Shock after heart transplantation and different type of cardiac operations, respectively. Noteworthy, survival and complications occurrence after central and peripheral cannulation were analyzed in a meta-analysis and this represent the unique attempt in the literature to address this issue. Outcome of ECMO use after complicated trans-aortic valve implantation were also reviewed. Lastly, the novel application of a pulmonary artery cannula in combination with ECMO support in patients with right or biventricular failure and acting both as drainage or inflow system were retrospectively analyzed. 
1. Stretch R, Sauer CM, Yuh DD, Bonde P. National trends in the utilization of short-term mechanical circulatory support. J Am Coll Cardiol 2014;64:1407-15

2. McCarthy FH, McDermott KM, Kini V, et al. Trends in U.S. extracorporeal membrane oxygenation use and outcome: 2002-2012. Sem Thorac Surg 2015;27:81-88

3. Sauer CM, Yuh DD, Bonde P. Extracorporeal membrane oxygenation use increased $433 \%$ in adults in the United States from 2006 to 2011. ASAIO J 2015:61:31-36

4. Maxwell BG, Powers AJ, Sheikh AY, Lee PHU, Lobato RL, Wong JK. Resource use trends in extracorporeal membrane oxygenation in adults: an analysis of the Nationwide Inpatient Sample 1998-2009. J Thorac Cardiovasc Surg 2014;148;416-21

5. Whitman GJR. Extracorporeal membrane oxygenation for the treatment of postcardiotomy shock. J Thorac Cardiovasc Surg 2016;153:95-101

6. Goldstein DJ, Oz MC. Mechanical support for postcardiotomy cardiogenic shock. Sem Thorac Cardiovasc Surg 2000;12:220-8

7. Curtis JJ, McKenney-Knox CA, Wagner-Mann CC. Postcardiotomy centrifugal assist: a single surgeon's experience. Artif Organs 2002;26:994-7

8. Jurmann MJ, Siniawski H, Erb M, Drews T, Hetzer R. Initial experience with miniature axial flow ventricular assist devices for postcardiotomy heart failure. Ann Thorac Surg 2004;77:1642-7

9. Hernandez AF, Grab JD, Gammie JS, et al. A decade of short-term outcomes in post-cardiac surgery ventricular assist device implantation. Circulation 2007;116:606-12

10. Akay MH, Gregoric ID, Radovancevic R, Cohn WE, Frazier OH. Timely use of a CentriMag heart assist device improves survival in postcardiotomy cardiogenic shock. J Card Surg $2011 ; 26: 548-52$

11. Sylvin EA, Stern DR, Goldstein DJ. Mechanical support for postcardiotomy cardiogenic shock: has progress been made? J Card Surg 2010;25:442-54 
12. Fukuhara S, Takeda K, Garan A, et al. Contemporary mechanical circulatory support therapy for postcardiotomy shock. Gen Thorac Cardiovasc Surg 2016;64:183-91

13. Magovern GJ Jr, Magovern JA, Benckart DH, et al. Extracorporeal membrane oxygenation: preliminary results in patients with postcardiotomy cardiogenic shock. Ann Thorac Surg $1994 ; 57: 71$

14. Kodera K, Kitamura M, Hachida M, Endo M, Hashimoto A, Koyanagi H. Biventricular bypass with oxygenation for postcardiotomy ventricular failure. Artif Organs 1996;20:724-7

15. Fiser SM, Tribble CG, Kaza AK, et al. When to discontinue extracorporeal membrane oxygenation for postcardiotomy support. Ann Thorac Surg 2001;71:210-4

16. Smedira NG, Blackstone EH. Postcardiotomy mechanical support: risk factors and outcomes. Ann Thorac Surg 2001;71:S60-6

17. Ko WJ, Lin CY, Chen RJ, Wang SS, Lin FY, Chen YS. Extracorporeal membrane oxygenation support for adult postcardiotomy cardiogenic shock. Ann Thorac Surg $2002 ; 73: 538-45$

18. Zhang R, Kofidis $\mathrm{T}$, Kamiya $\mathrm{H}$, et al. Creatine kinase isoenzyme MB relative index as predictor of mortality on extracorporeal membrane oxygenation support for postcardiotomy cardiogenic shock in adult patients. Eur J Cardio-Thorac Surg 2006;30:617-20

19. Bakhtiary F, Keller H, Dogan S, et al. Venoarterial extracorporeal membrane oxygenation for treatment of cardiogenic shock : clinical experiences in 45 adult patients. J Thorac Cardiovasc Surg $2008 ; 135: 382-8$

20. Hsu PS, Chen JL, Hong GJ, et al. Extracorporeal membrane oxygenation for refractory cardiogenic shock after cardiac surgery: predictors of early mortality and outcome from 51 patients. Eur J Cardio-Thorac Surg 2010;37:328-33

21. Rastan AJ, Dege A, Mohr M, et al. Early and late outcome of 517 consecutive patients treated with extracorporeal membrane oxygenation for refractory postcardiotomy cardiogenic shock. J Thorac Cardiovasc Surg 2010;139:302-11 
22. Elsharkawy HA, Li L, Skr Esa WA, Sessler D, Bashour CA. Outcome in patients who require venoarterial extracorporeal membrane oxygenation support after cardiac surgery. J CardioThorac Vasc Anesth 2010;24:946-51

23. Pokersnik JA, Buda T, Bashour A, Gonzalez-Stawinski GV. Have changes in ECMO technology impacted outcomes in adult patients developing postcardiotomy cardiogenic shock? J Card Surg 2012;27:246-52

24. Mikus E, Tripodi A, Calvi S, Del Giglio M, Cavallucci A, Lamarra M. CentriMag venoarterial extracorporeal membrane oxygenation supporta $\mathrm{s}$ treatment for patients with refractory postcardiotomy cardiogenic shock. ASAIO J 2013:59:18-23

25. Slottosch I, Liakopoulos O, Kuhn E, et al. Outcomes after peripheral extracorporeal membrane oxygenation therapy for postcardiotomy cardiogenic shock: a single centre experience. J Surg Res 2013;181:47-55

26. Unosawa S, Sezai A, Hata M, et al. Long-term outcomes of patients undergoing ECMO for refractory postcardiotomy cardiogenic shock. Surg Today 2013;43:264-70

27. Ariyaratnam P, McLean LA, Cale ARJ, Loubani M. Extracorporeal membrane oxygenation for the post-cardiotomy patient. Heart Fail Rev 2014;19:717-25

28. Li CL, Wang H, Jia M, Ma N, Meng X, Hou XT. The early dynamic behaviour of lactate is linked to mortality in postcardiotomy patients with extracorporeal membrane oxygenation support: a retrospective observational study. J Thorac Cardiovasc Surg 2015;149:1445-50

29. Saxena P, Neal J, Joyce LD, et al. Extracorporeal membrane oxygenation support in postcardiotomy elderly patients: the Mayo Clinic experience. Ann Thorac Surg 2015;99:205360

30. Khorsandi M, Shaikhrezai K, Prasad S, et al. Advanced mechanical circulatory support for post-cardiotomy cardiogenic shock: a 20 -year outcome analysis in a non-transplant unit. $J$ Cardio-Thorac Surg 2016;11:29-35 
31. Dilstelmaier K, Schrutka L, Binder C, et al. Cardiac arrest does not affect survival in postoperative cardiovascular surgery patients undergoing extracorporeal membrane oxygenation. Resuscitation 2016;104:24-7

32. Mazzeffi MA, Sanchez PG, Herr D, et al. Outcomes of extracorporeal cardiopulmonary resuscitation for refractory cardiac arrest in adult cardiac surgery patients. J Thorac Cardiovasc Surg 2016;152:1133-9

33. Chen SW, Tsai FC, Lin YS, et al. Long-term outcomes of extracorporeal membrane oxygenation support for postcardiotomy shock. J Thorac Cardiovasc Surg 2017;154:469-77

34. Guihaire J, Dang Van S, Rouze S, et al. Clinical outcomes in patients after extracorporeal membrane oxygenation support for post-cardiotomy cardiogenic shock: a single-centre experience. Interact Cardiovasc Thorac Surg 2017, doi: 10.1093/icvts/ivx155.

35. Raffa GM, Gelsomino S, Sluijpers N, et al. In-hospital outcome of post-cardiotomy extracorporeal life support in adult patients: the 2007-2017 Maastricht experience. Crit Care Resusc. 2017;19:53-61.

36. Fux, T, Holm M, Corbascio M, H. Lund LH, van der Linden J. Venoarterial Extracorporeal Membrane Oxygenation for Postcardiotomy Shock: Risk Factors for Mortality. J Thorac Cardiovasc Surg 156;5:1894-1902.e3, doi.org/10.1016/j.jtcvs.2018.05.061.

37. Ranucci M, Ballotta A, Kandil H, et al. Bivalirudin-based versus heparin anticoagulation for postcardiotomy extracorporeal membrane oxygenation. Critical Care 2011;15:275-85

38. Ranucci M, Barushnikova E, Isgrò G, et al. Heparin-like effect in post-cardiotomy extracorporeal membrane oxygenation patients. Critical Care 2014;18:504-10

39. Smith C, Bellomo R, Raman JS, et al. An extracorporeal membrane oxygenation-based approach to cardiogenic shock in an older population. Ann Thorac Surg 2001;71:1421-7

40. Saito S, Nakatani T, Kobayashi J, et al. Is extracorporeal life support contraindicated in elderly patients? Ann Thorac Surg 2007;83:140-5 
41. Lan C, Tsai PR, Chen YS, Ko WJ. Prognostic factors for adult patients receiving extracorporeal membrane oxygenation as mechanical circulatory support - A 14-year experience at a medical center. Artif Organs 2010;34:59-64

42. Hei F, Lou S, Li J, et al. Five-year results of 121 consecutive patients treated with extracorporeal membrane oxygenation at Fu Wai hospital. Artif Organs 2011;35:572-8

43. Raffa GM, Kowalewski M, Brodie D, et al. Meta-Analysis of Peripheral or Central ECMO in Postcardiotomy and Non-Postcardiotomy Shock. Ann Thorac Surg. 2018, doi: 10.1016/j.athoracsur.2018.05.063.

44. LoforteA, Marinelli G, Musumeci F, et al. Extracorporeal membrane oxygenation support in refractory cardiogenic shock: treatment strategies and analysis of risk factors. Artif Organs 2014;38:129-41

45. Saed D, Stosik H, Islamovic M, et al. Femoro-femoral versus atrio-aortic ECMO: selecting the ideal cannulation technique. Artif Organ 2014;38:549-55

46. Carroll BJ, Shah RV, Murthy V, et al. Clinical features and outcomes in adults with cardiogenic shock supported with extracorporeal membrane oxygenation. Am J Cardiol 2015;116:1624-30

47. Truby L, Mundy L, Kalesan B, et al. Contemporary outcomes of venoarterial extracorporeal membrane oxygenation for refractory cardiogenic shock at a large tertiary care center. ASAIO $J$ 2015;61:403-9

48. Brunet J, Valette X, Ivascau C, et al. Extracorporeal life support for refractory cardiac arrest or shock: a 10-year study. ASAIO J 2015;61:676-81

49. Tarzia V, Bertolussi G, Bianco R, et al. Extracorporeal life support in cardiogenic shock: impacts of acute versus chronic etiology on outcome. J Thorac Cardiovasc Surg $2015 ; 150: 333-40$ 
50. Narotsky DL, Mosca MS, Mochari-Greenberger H, et al. Short-term and long-term survival of veno-arterial extracorporeal membrane oxygenation in an adult patient population: does older age matter? Perfusion 2016;31:366-75

51. Aso S, Matsui H, Fushimi K, Yasunaga H. In-hospital mortality and successful weaning from veno-arterial extracorporeal membrane oxygenation: analysis of 5,263 patients using a national inpatient database in Japan. Critical Care 2016;20-80

52. Demodion P, Fournel L, Golmard JL, Niculescu M, Pavie A, Leprince P. Predictors of 30day mortality and outcome in cases of myocardial infarction with cardiogenic shock treated by extracorporeal membrane oxygenation. Eur J Cardio-Thorac Surg 2014;45:47-54

53. Takayama H, Truby L, Koekort M, et al. Clinical outcome of mechanical circulatory support for refractory cardiogenic shock in the current era. J Heart Lung Transplant 2013;32:106-11

54. Xie A, Phan K, Tsai YC, Yan TD, Forrest P. venoarterial extracorporeal membrane oxygenation for cardiogenic shock and cardiac arrest: a metanalysis. J Cardio-Thorac Vasc Anesth 2015;29:637-45

55. Nakamura H, Yamaguchi H, Amano A, Nakao T. Veno-venous extracorporeal membrane oxygenation is effective against post-cardiotomy acute respiratory failure in adults. Gen Thorac Cardiovasc Surg 2013;61:402-8

56. Song JH, Woo WK, Song SH, et al. Outcome of veno-venous extracorporeal membrane oxygenation use in acute respiratory distress syndrome after cardiac surgery with cardiopulmonary bypass. J Thorac Dis 2016;8:1804-13

57. Chou NK, Chi NH, Wu IW, et al. Extracorporeal membrane oxygenation to rescue cardiopulmonary failure after heart transplantation: a single-centre experience. Transplant Proceed 2010;42:943-5

58. Borges Lima E, da Cunha CR, Barzilai VS, et al. Experience of ECMO in primary graft dysfunction after orthotopic heart transplantation. Arq Bras Cardiol 2015;105:285-91 
59. D’Alessandro C, Aubert S, Golmard JL, et al. ECMO temporary support for early graft failure after cardiac transplantation. Eur J Cardio-Thorac Surg 2010;37:343-9

60. Kittleson MM, Patel J, Moriguchi J, et al. Heart transplant recipients supported with ECMO: outcomes from a single-centre experience. J Heart Lung Transplant 2011;30:1250-6

61. Listijono D, Watson A, Pye R, et al. Usefulness of ECMO for early cardiac allograft dysfunction. J Heart Lung Tranplant 2011;30:783-9

62. Dandel M, Krabatsch T, Valk F. Left ventricular versus biventricular mechanical support: decision making and strategies for avoidance of right heart failure after left ventricular assist device implantation. Int J Cardiol 2015;198:241-50

63. Haneya A, Philipp A, Puehle T, et al. Temporary percutaneous right ventricular support using a centrifugal pump in patients with postoperative acute refractory right ventricular failure a er le ventricular assist device implantation. Eur J Cardiothorac Surg 2012;41:219-23

64. Argiriou M, Kolokotron SM, Sakellaridis T, et al. Right heart failure post left ventricular assist device implantation. J Thorac Dis 2014;6:552-9

65. Zhao Y, Xing J, Du Z, Liu F, Hou X. Extracorporeal cardiopulmonary resuscitation for adult patients who underwent post-cardiac surgery. Eur J Med Res 2016;20:83-9

66. Society of Thoracic Surgeons Task Force on Resuscitation After Cardiac S. The Society of Thoracic Surgeons Expert Consensus for the Resuscitation of Patients Who Arrest After Cardiac Surgery. Annals Thorac Surg 2017;103:1005-20

67. Navia J, Atik FA, Beyer EA, Ruda Vega P. Extracorporeal membrane oxygenation with right axillary artery perfusion. Ann Thorac Surg 2005;79:2163-5

68. Biscotti M, Bacchetta M. The "Sport Model”: extracorporeal membrane oxygenation using the subclavian artery. Ann Thorac Surg 2014;98:1487-9

69. Ranney DN, Benrashid E, Meza JM, et al. Central cannulation as a viable alternative to peripheral cannulation in extracorporeal membrane oxygenation. Sem Thorac Cardiovasc Surg 2017;29:188-95 
70. Babu A. Techniques of venoarterial ECMO support and conversion to temporary LVAD. $O p$ Tech Thorac Cardiovasc Surg 2014;19:365-79

71. Meani P, Gelsomino S, Natour E, et al. Modalities and effects of left ventricular unloading on extracorporeal life support: a review of the literature. Eur J Heart Fal 2017;19:81-88

72. Pappalardo F, Schulte C, Pieri M, et al. Concomitant uimplantation of Impella on top of VAECMO may improves survival of patients with cardiogenic shock. Eur J Heart Fail $2017 ; 19: 404-12$

73. Tepper S, Faraz Masood M, Baltazar Garcia M, et al. Left ventricular unloading by Impella device versus surgical vent during extracorporeal life support. Ann Thorac Surg $2017 ; 104: 861-7$

74. Cheng R, Hachamovitch R, Makkar R, et al. Lack of survival benefit found with th use of intra-aortic balloon pump in ECMO: a pooled experience of 1517 patients. J Invas Cardiol $2015 ; 27: 453-8$

75. Nuding S, Werdan K. IABP plus ECMO - Is one and one more than two? $J$ Thorac Dis 2017;9:961-4

76. Pappalardo F, Montisci A. Veno-arterial extracorporeal membrane oxygenation (VA-ECMO) in post-cardiotomy cardiogenic shock: how much pump flow is enough? J Thorac Dis 2016;8:1444-8

77. Haft JW. Temporary mechanical circulatory support for post-cardiotomy shock: don't come late to the party. J Thorac Cardiovasc Surg 2015;149:1451-2

78. Dilstelmaier K, Roth C, Schrutka L, et al. Beneficial effects of levosimendan on survival in patients undergoing extracorporeal membrane oxygenation after cardiovascular surgery. Brit J Anesth 2016;117:52-8

79. Affronti A, di Bella I, Carino D, Ragni T. Levosimendan may improve weaning outcomes in venoarterial ECMO patients. ASAIO J. 2013;59:554-7 
80. Cheng R, Hachamovitch R, Kittleson M, et al. Complications of extracorporeal membrane oxygenation for treatment of cardiogenic shock and cardiac arrest: a meta-analysis of 1,866 adult patients. Ann Thorac Surg 2014;97:610-6

81. Lorusso R, Barili F, Di Mauro M, et al. In-hospital neurologic complications in adult patients undergoing venoarterial extracorporeal membrane oxygenation: Results from the Extracorporeal Life Support Organization Registry. Crit Care Med 2016;44:e964-e972

82. Lorusso R, Gelsomino S, Parise O, et al. Neurologic injury in adults supported with venovenous ECMO for respiratory failure: findings from the Extracorporeal Life Support Organization Registry. Crit Care Med 2017;45:1389-97

83. Wong JK, Smith TN, Pitcher HT, Hirose H, Cavarocchi NC. Cerebral and lower limb near infra-red spectroscopy in adults on extracorporeal membrane oxygenation. Artif Organs. 2012;36:659-67

84. Rastan AJ, Lachmann N, Walther T, et al. Autopsy findings in patients on postcardiotomy ECMO. Int J Artif Organs 2006;29:1121-31

85. Marasco SF, Vale M, Pellegrino V, et al. Extracorporeal membrane oxygenation in primary graft failure after heart transplant. Ann Thorac Surg 2010;90:1541-7

86. Takeda K, Li B, Garan AR, et al. Improved outcome from ECMO versus ventricular assist device temporay support of primary graft dysfunction after heart transplant. Heart Lung $2017 ; 36: 640-6$

87. Lorusso R, Gelsomino S, Parise O, et al. Veno-Arterial Extracorporeal Membrane Oxygenation for Refractory Cardiogenic Shock in Elderly Patients: Trends in Application and Outcome from the Extracorporeal Life Support Organization (ELSO) Registry. Ann Thorac Surg 2017;104:62-9

88. Yan X, Jia S, Meng X, et al. Acute kidney injury in adult postcardiotomy patients with extracorporeal membrane oxygenation: evaluation of the RIFLE classification and the Acute Kidney Injury Network criteria. Eur J Cardio-Thorac Surg 2010;37:334-8 
89. Roth C, Schrutka L, Binder C, et al. Liver function predicts survival in patients undergoing extracorporeal membrane oxygenation following cardiovascular surgery. Crit Care 2016;20:57

90. Dilstelmaier K, Winter MP, Rutzler K, et al. Serum butyrylcholiesterase predicts survival after extracorporeal membrane oxygenation after cardiovascular surgery. Crit Care 2014;18:R24

91. Rahmanian PB, Adams DH, Castillo JG, Carpentier A, Filsoufi F. Predicting hospital mortality and analysis of long-term survival after major non-cardiac complications in cardiac surgery patients. Ann Thorac Surg 2010;90:1221-9

92. Stephens RS, Shah AS, Whitman GJR. Lung injury and acute respiratory distress syndrome after cardiac surgery. Ann Thorac Surg 2013;95:1122-9

93. Peek G, Mugford M, Tiruvoipati R, et al. Efficacy and economic assessment of conventional ventilator support versus extracorporeal membrane oxygenation for severe adult respiratory failure (CESAR): a multicentre randomised controlled trial. Lancet 2009;374:1351-63

94. Schmidt M, Bailey M, Sheldrake J, et al. Predicting survival after ECMO for severe acute respiratory failure. Am J Respir Crit Care Med 2014;189:1374-8 
CHAPTER 2

\section{Heart Transplant Program at IRCCS-ISMETT:}

Impact of Mechanical Circulatory Support on Pre- and Post -Transplant Survival

Raffa GM, Di Gesaro G, Sciacca S, Tuzzolino F, Turrisi M, Falletta C, Minà C, Romano $G$, Vitale $G$, Panarello $G$, Clemenza F, Pilato $M$.

International Journal of Cardiology 2016 Sep 15;219:358-61. 


\section{Abstract}

Background. Heart transplantation (HTx) improves the quality of life and survival in patients affected by end-stage heart failure. The purpose of the current study is to present the patients' clinical data and results of HTx in a single Center of Sicily. Focus on survival after pre and post HTx mechanical circulatory support use will be performed.

Methods. 133 HTx were done from 2004 to the end of 2015.The average donor age was $34 \pm 13.5$ years and the proportion of male donors was $67 \%$. Percentage of use of mechanical circulatory support to bridge patients to HTx was $18 \%$.

Results. Overall pre-transplant mechanical circulatory support was not correlated to worse posttransplant prognosis, $\mathrm{p}=0.757$. Severe primary early graft failure requiring extra corporeal membrane oxygenator support strongly impact the early mortality after heart transplantation $(\mathrm{p}<0.001)$.

Conclusions. The results of HTx at ISMETT are comparable to those reported in high volume Italian transplant centers as well as in the ISHLT registry. The favorable outcome can be related to focus on multidisciplinary approach, strict recipients' selection and young donor population. Post HTx mechanical circulatory support use in general remains associated with worse post-transplant outcomes. This does not apply to pre-op mechanical circulatory support population. 
Heart transplantation (HTx) improves the quality of life and survival in patients affected with endstage heart disease that is not treatable with more conservative measures [1]. The first HTx at ISMETT (Istituto Mediterraneo per i Trapianti e Terapie ad alta specializzazione) was performed on October 20, 2004, and by the end of 2015, the institute had done one hundred thirty-three HTx.

The purpose of this report is to present the patients' clinical data, and the results of HTx at ISMETT. Focus on pre and post HTx mechanical circulatory support (MCS) use will be analyzed.

\section{Methods}

The study was approved by the IRRB of the "Istituto Mediterraneo per i Trapianti e Terapie ad Alta Specializzazione”, IRCCS-ISMETT).

Statistical analysis. Data were collected retrospectively, and continuous and categorical variables are expressed as the mean $\pm \mathrm{SD}$, and as percentage, respectively. The Kaplan-Meier method and the log rank test were used to estimate the survival.

Procurement and transplantation. Celsior cardioplegic solution was used for the procurement of the donor hearts, with the infusion of one liter of solution to achieve the plegic arrest, and one liter in the bag for the transport. Most of the transplants were performed using the bicaval technique.

\section{Results}

Heart transplant donor and recipient characteristics. Donor demographics for adult recipients since 2004 are presented in Table 1 . The mean donor age was $34 \pm 13.5$ years, and the proportion of male donors was $67 \%$. The leading cause of donor death was head trauma (52.7\%). The average male and female recipient age was $50 \pm 11$ years and $45 \pm 11$ years, respectively. Approximately $85 \%$ of primary transplant recipients were male. Coronary artery disease (62 patients, 47\%) and cardiomyopathy (39 patients, 29\%) were overwhelmingly the leading underlying heart disease diagnoses (Fig. 1). 


\section{Etiology}

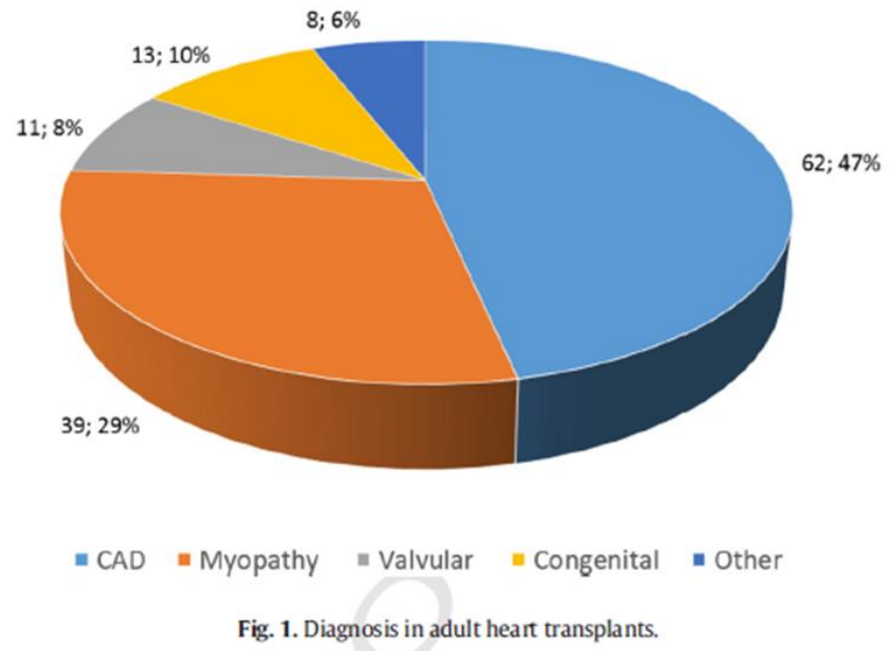

Percentage of use of MCS by means of left- (LVAD) or bi-ventricular assist device and extracorporeal membrane oxygenation to bridge patients to transplant was 18\%. Details of HeartWare® (HeartWare Inc., Framington, MA) continuous flow left ventricle assist device experience performed at IRCCSISMETT have been previously published [2-4]. Pre-operative intra-aortic balloon pump (IABP) use was $8.2 \%$. The average ischemic time was $173.2 \pm 55.4$ minutes $(\leq 120$ minutes in 29 patients $[21.8 \%]$; $>120$ minutes and $<180$ minutes in 40 patients [30\%]; $\geq 180$ minutes and $\leq 240$ minutes in 49 patients [36.9\%], and > 240 minutes in 15 patients [11.3\%]). No retransplantation has been performed at out institute. 
Table 1

Recipient and donor tranglant characteristic for aduk heart transplants

\begin{tabular}{|c|c|}
\hline Dona variables & All $(n=133)$ \\
\hline Age, yean & $34=13.5$ \\
\hline Cender, $\mathrm{x}$ male & $66.9(n=89)$ \\
\hline BML kgm2 & $25.2 \pm 3.4$ \\
\hline \multicolumn{2}{|l|}{ Cause of death $x$} \\
\hline Head trauma & $52.7(\mathrm{n}=\mathbf{D})$ \\
\hline Stroke, bemontagic & $38.4(n=50)$ \\
\hline Strole, ischemic & $37(n=5)$ \\
\hline Other & $52(\mathrm{n}=7)$ \\
\hline \multicolumn{2}{|l|}{ Blood type, $\mathbf{X}$} \\
\hline 0 & $55.6(n=74)$ \\
\hline A & $31.6(n=2)$ \\
\hline B & $12.8(n=17)$ \\
\hline \multicolumn{2}{|l|}{ Recruitment, $x$} \\
\hline Sicily & $54.8(n=73)$ \\
\hline Italy & $4.3(n=98)$ \\
\hline Europe & $09(\mathrm{n}=1)$ \\
\hline \multicolumn{2}{|l|}{ Recipient varubles } \\
\hline Age years & $8.9 \pm 11$ \\
\hline Cender, $\mathbf{x}$ male & $84.9(n=113)$ \\
\hline BMI kg/m2 & $\mathbf{x}=3$ \\
\hline \multicolumn{2}{|l|}{ Bbod type, $x$} \\
\hline 0 & $5.8(n=61)$ \\
\hline A & $38.9(n=4)$ \\
\hline B & $15,7(n=21)$ \\
\hline$A B$ & $46(n=6)$ \\
\hline Diabetes, $x$ & $23.3 x(n=31)$ \\
\hline Creatinine at time of transplant, ng/dl & $1.3 \pm 0.3$ \\
\hline COID & $3(n=4)$ \\
\hline Previbus cardix surgery & $38.3(n=51)$ \\
\hline Pumanary vascular resistance, wU & $21 \pm 13$ \\
\hline Cardia resynchron bation ther apy & $24.8(n=33)$ \\
\hline Implantable cardoverter defitrabtors & $71.4(n=9)$ \\
\hline MCS & $18(\mathrm{n}=24)$ \\
\hline HeartWare LVAD & $33.4(n=8)$ \\
\hline Thoratec LVAD & $83(n=2)$ \\
\hline Levituonix LVAD & $8.3(n=2)$ \\
\hline Levitronix BiVAD & $\mathbf{b}(n=6)$ \\
\hline ECMO & $2(n=6)$ \\
\hline HBP & $82(n=11)$ \\
\hline \multicolumn{2}{|l|}{ Clinical status } \\
\hline 1 & $12.7(n=n)$ \\
\hline $2 a$ & $12(n=16)$ \\
\hline $2 b$ & $75.3(n=100)$ \\
\hline Male recipient/female donor & 39 \\
\hline Fenale recipient/male donor & 4 \\
\hline kchernic time, minutes & $172 \pm 55$ \\
\hline
\end{tabular}

BMI:body mass index; COPD:chronic obstructive pulmonary disease; MCS: mechanical circulatory support; LVAD: left ventricular assist device; ECMO: extra corporeal membrane oxygenation; LABP:intra-aortic balloon pump.

Early graft failure. Early graft failure (EGF) was defined and classified according to the recent International Society for Heart and Lung Transplantation criteria [5]. The overall EGF rate was 40\%, and the primary and secondary EGF rate was $30 \%$ and $10 \%$, respectively. Pulmonary hypertension (57.1\%) and bleeding (21.4\%) were the major causes of secondary EGF. The high inotropic score [5] and the IABP use after HTx defined the moderate EGF (21.6\%). The rate of severe EGF, requiring circulatory assistance, was $18.4 \%$. ECMO was the support of choice, utilized in all cases as bridge to recovery. ECMO cannulation was done either peripherally through the femoral vessels or centrally 
by sternotomy. ECMO support was weaned in $84 \%$ of cases. Patients on ECMO were treated according to our institutional protocols [6], with 1) low dose of adrenaline (0.05 mic/kg per minute) to optimize residual cardiac function; 2) femoral IABP placement to allow aortic valve opening, decompressing the left ventricle, and increasing coronary flow; 3) low dose of vasoconstrictor (noradrenaline or vasopressin), and careful fluid balance to avoid fluid overload; and 4) transesophageal echocardiography monitoring of aortic valve opening, mitral regurgitation, and left ventricle distention [6].

Survival. For all 133 adult heart transplants, 1-year survival was $83 \%$, and 5-year survival was $81 \%$. Hospital mortality was $14.2 \%$ (19/133). Overall pre-transplant MCS was not correlated with worse post-transplant prognosis, $\mathrm{p}=0.757$ (Fig. 2). Both pre-operative ECMO and LVAD use did not affect post-transplant survival ( $\mathrm{p}=0.234$ and $\mathrm{p}=0.143$, respectively (Fig. 2). 

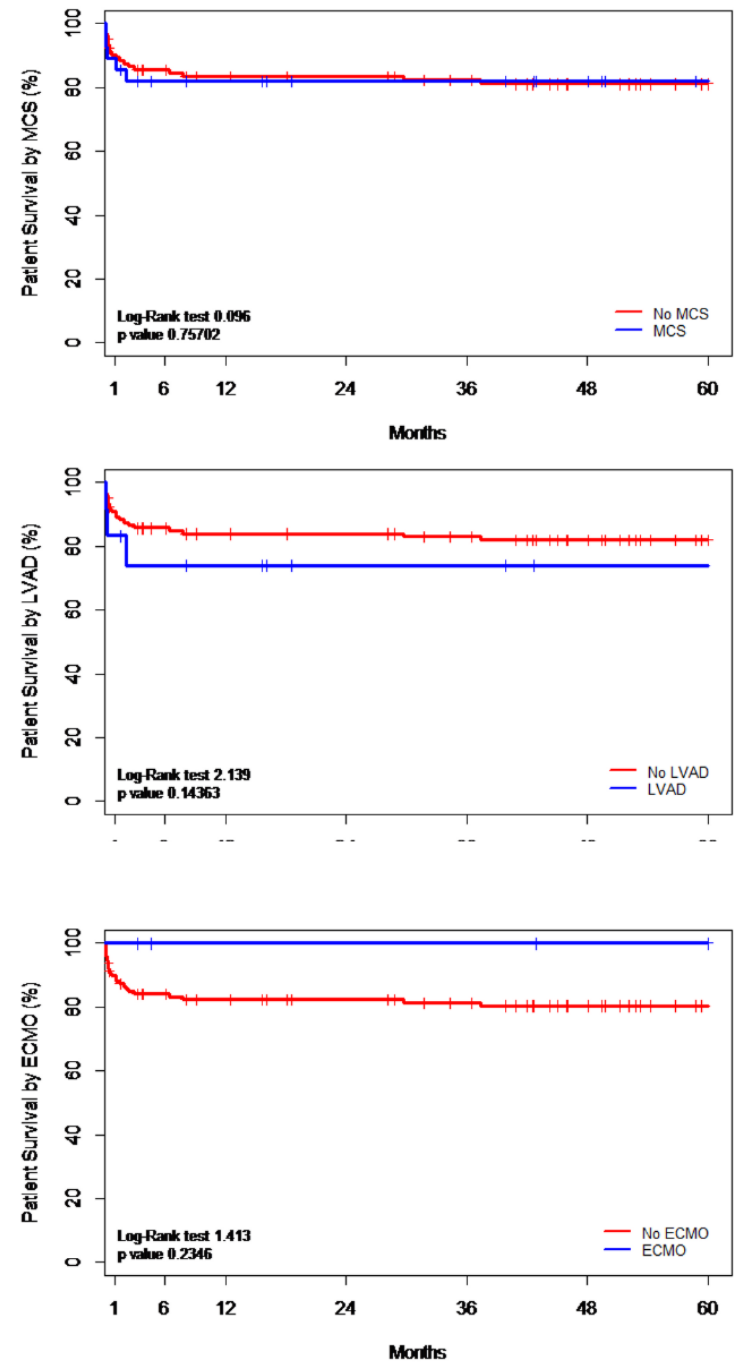

Fig. 2. Survival according to pre-transplant mechanical circulatory support (MCS), left ventricle assist device (LVAD) and extra corporeal membrane oxygenator (ECMO). MCS includes HeartWare, Levitronix, Thoratec, extra corporeal membrane oxygenator, and the intra-aortic balloon pump use.

Severe primary EGF strongly impacted early mortality after heart transplantation ( $<<0.001$, Fig. 3). 

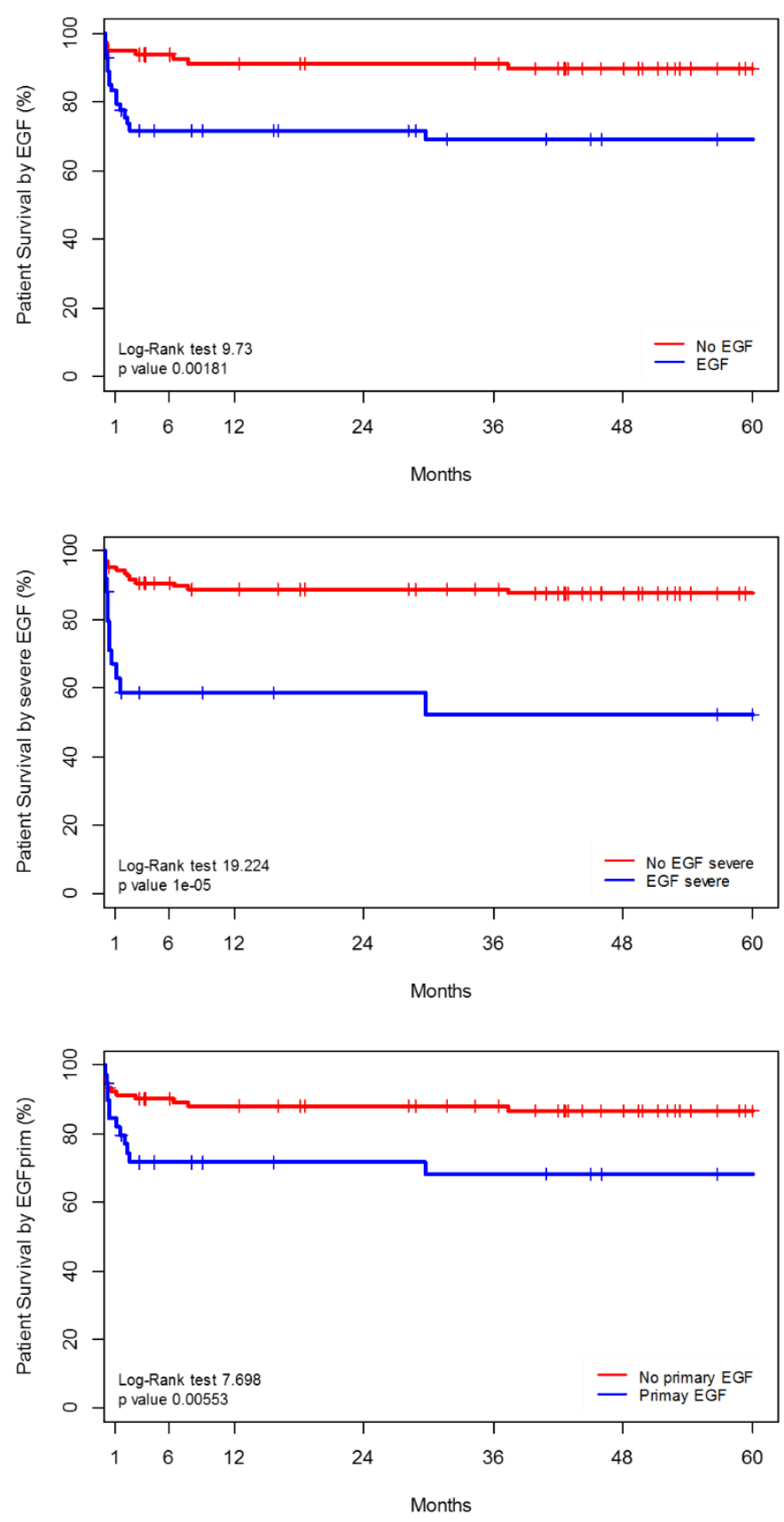

Fig 3. Survival according to early graft failure (EGF) occurrence. Severe and primary EGF was defined and classified according to the recent International Society for Heart and Lung Transplantation (ISHLT) criteria [5].

Excluding the 90-day mortality, the survival between patients with EGF and those without was similar ( $\mathrm{p}=0.693$, Fig. 4). 

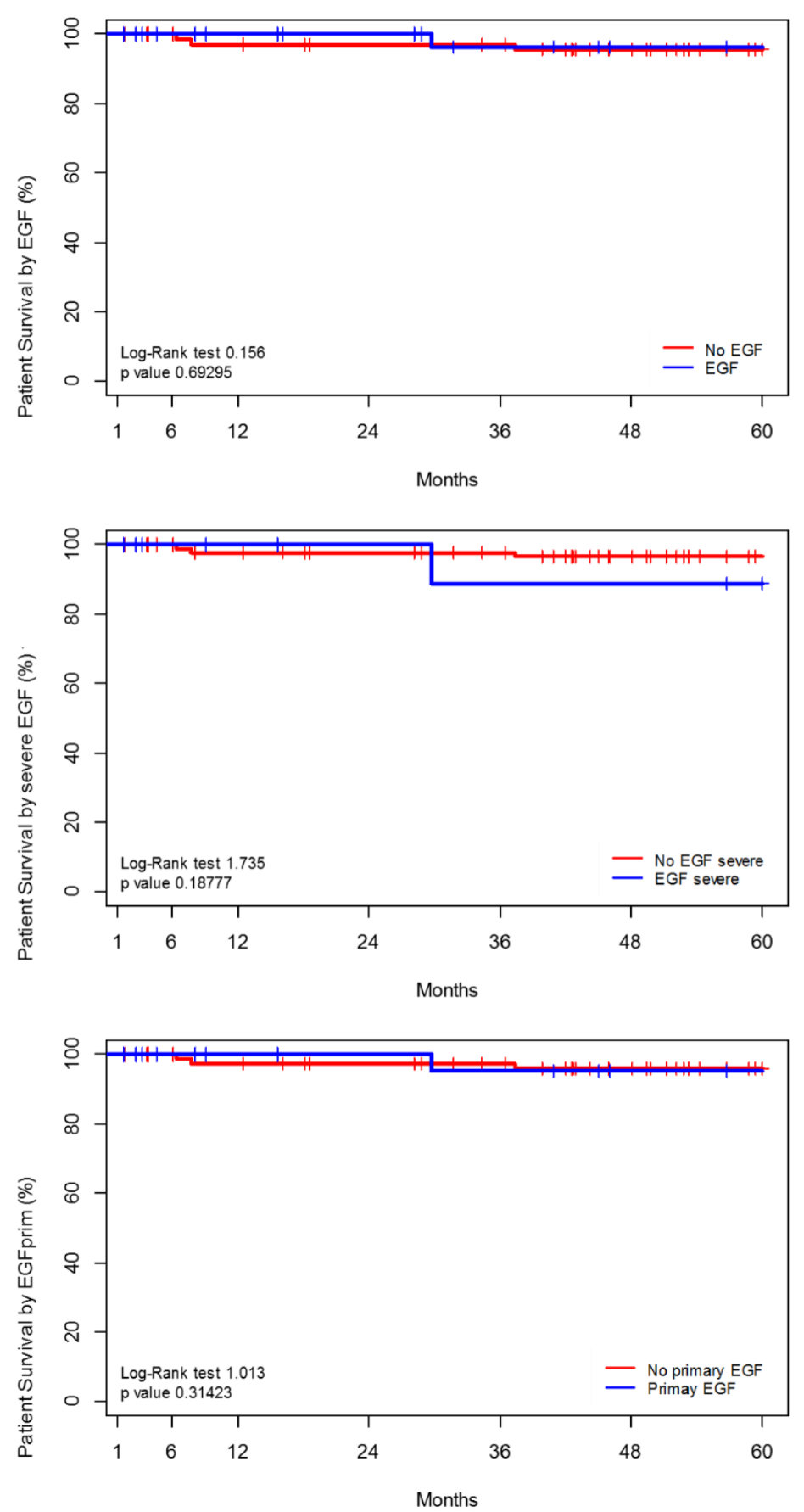

Fig. 4. Survival according to early graft failure (EGF) excluding the 90 days mortality. Severe and primary EGF was defined and classified according to the recent International Society for Heart and Lung Transplantation (ISHLT) criteria [5].

Both EGF and mortality risk of patients receiving an "out of region organ" (longer ischemic time, often greater than 240 minutes [7]) were higher $(\mathrm{p}<0.001)$ when compared with those receiving an organ procured in Sicily (Table 1). 
Cause of death. The leading causes of death for all transplant recipients were infections (41.9\%), multiple organ failure (22.5\%), and irreversible acute graft failure (9.6\%), while malignancy (9.6\%) and arrhythmia/sudden death became progressively more important after 3 to 6 years. Eight patients suffering from severe EGF in which ECMO support was weaned died because of infections $(5 / 8$ patients) and multi organ failure (3/8 patients).

Immunosuppression and rejection. Immunosuppressive induction strategies included anti-thymocyte globulin or basiliximab according to date of transplant. Since 2014, induction is no longer routinely used at our institute. Maintenance immunosuppression included tacrolimus as the preferred calcineurin inhibitor, and mycophenolate mofetil/mycophenolic acid as the preferred cell-cycle inhibitor. The incidence of any rejection requiring acute treatment and hospitalization [8, 9] was $13.2 \%$.

Morbidity. Hypertension (21.4\%), renal dysfunction (14.8\%), diabetes (2.4\%), and cardiac allograft vasculopathy $(\mathrm{CAV}, 7.55 \%)$ were the most common post-transplant morbidities. Incidence of malignancy was $9 \%$ and included lung, kidney, and skin cancer (1.6\% each) and post-transplant lymphoproliferative disorders $(2.4 \%)$.

\section{Discussion}

The results of HTx at ISMETT are comparable to those reported in high volume Italian transplant centers [10], as well as in the ISHLT registry [1]. The favorable outcomes could be attributed to our focus on a multidisciplinary approach, strict selection and work-up of recipients, and a young donor population. Head trauma, the most frequent cause of donor death in our series, may also play a role in the long-term outcome [1]. However, the longer ischemic time for organs procured outside of Sicily increased the EGF rate and early mortality risk of HTx recipients. Post-op MCS use, in general, is associated with worse post-transplant outcomes [1], while association with worse outcomes with preop use is a matter of controversy. In our series, pre-op MCS use, with application of a continuous 
flow device, and our experience in the management of patients supported with ECMO before and after HTx [6] resulted in a lower risk of adverse outcome.

CAV remains a major cause of long-term morbidity and mortality after HTx [1], though in our series there was a low rate because of the relatively short follow-up time.

In conclusion, HTx is the most successful and effective method of managing patients with end-stage heart failure refractory to conventional therapies. Excellent results can be achieved even in centers with low to moderate HTx volumes. Wise application of new MCS technologies, both pre- and posttransplant, may improve the results, allowing a bridge to transplantation for unstable patients or a bridge to recovery and survival after severe EGF. 


\section{References}

1. Lund LH, Edwards LB, Kucheryavaya AY, et al. The Registry of the International Society for Heart and Lung Transplantation: Thirty-second Official Adult Heart Transplantation Report-2015; Focus Theme: Early Graft Failure. J Heart Lung Transplant. 34 (2015) 1244-54.

2. Romano G, Raffa GM, Ruggieri A, et al. Recurrent ventricular tachycardia in a patient with continuous flow left ventricle assist device: Successful management with radiofrequency ablation and medical treatment. Int J Cardiol. 190 (2015) 198-200.

3. Raffa GM, D'Ancona G, Romano G, et al. Should device replacement be the first choice strategy in continuous-flow left ventricle assist device thrombosis? Analysis of 9 events and results after endoventricular thrombolysis. Int J Cardiol. 178 (2015) 159-61.

4. Raffa GM, D'Ancona G, Sciacca S, et al. Vascular access complications in patients with continuousflow left ventricle assist device undergoing percutaneous invasive procedures: a word of caution. Int J Cardiol. 174 (2014) 768-9.

5. Kobashigawa J, Zuckermann A, Macdonald P, et al. Report from a consensus conference on primary graft dysfunction after cardiac transplantation. J Heart Lung Transplant. 33 (2014) 327-40.

6. Santise G, Panarello G, Ruperto C, et al. Extracorporeal membrane oxygenation for graft failure after heart transplantation: a multidisciplinary approach to maximize weaning rate. Int J Artif Organs. 37 (2014) 706-14.

7. Segovia J, Cosío MD, Barceló JM, et al. RADIAL: a novel primary graft failure risk score in heart transplantation. J Heart Lung Transplant. 30 (2011) 644-51.

8. Stewart S, Winters GL, Fishbein MC, et al. Revision of the 1990 working formulation for the standardization of nomenclature in the diagnosis of heart rejection. J Heart Lung Transplant. 24 (2005) 1710- 1720.

9. Romano G, Raffa GM, Licata P, et al. Can multiple previous treatment-requiring rejections affect biventricular myocardial function in heart transplant recipients? A two-dimensional speckle-tracking study. Int J Cardiol. 209 (2016) 54-6. 
10. Bruschi G, Colombo T, Oliva F, et al. Heart transplantation: 25 years' single-centre experience. J Cardiovasc Med (Hagerstown). 14 (2013) 637-47. 


\section{CHAPTER 3}

In-Hospital Outcome of Post-Cardiotomy Extracorporeal Life Support in Adult Patients:

the 2007-2017 Maastricht Experience

Raffa GM, Gelsomino S, Sluijpers N, Meani P, Alenizy K, Natour E, Bidar E, Johnson DM, Makhoul M, Heuts S, Lozekoot P, Kats S, Schreurs R, Delnoij T, Montalti A, Sels JW, Poll MV, Roekaerts P, Maessen J, Lorusso R.

Critical Care and Resuscitation. 2017 Oct;19(Suppl 1):53-61. 


\begin{abstract}
Objectives. The use of post-cardiotomy extracorporeal life support (PC-ECLS) has increased worldwide over the last years, however a concurrent decrease in survival to hospital discharge has also been observed. Use and outcome of PC-ECLS at the Maastricht University Medical Center (MUMC+) was analysed.
\end{abstract}

Design. A retrospective study of a single-centre PC-ECLS cohort was performed. Patient characteristics and in-hospital outcomes were evaluated.

Setting. Patients who underwent PC-ECLS due to intra- or peri-operative cardiogenic shock or cardiac arrest were included. Descriptive statistics, frequency analysis, and testing of group differences were performed.

Participants. Eighty-six patients who received PC-ECLS between October 2007 and June 2017 were included. Mean age of the population was 65 years (range 31-86 years), $65 \%$ were male.

Main outcome measures. Survival and bleeding rates were calculated. PC-ECLS management data and complications were also assessed.

Results. Pre-ECLS procedures were isolated coronary artery bypass grafting (CABG) (22\%), isolated valve surgery (16\%), thoracic aorta surgery (4\%), a combination of CABG and valve surgery (21\%) or other surgery (24\%). PC-ECLS was achieved via central cannulation in $17 \%$, peripheral cannulation in $65 \%$, or by a combination in $17 \%$. Median PC-ECLS duration was 5.0 days (IQR 6.0). Weaning was achieved in $49 \%$ of patients, whilst $37 \%$ survived to discharge. Postoperative bleeding (overall rate $42 \%$ ) showed a trend towards a reduced rate over more recent years.

Conclusions. Our experience confirms an increased use of PC-ECLS during the last 10 years and shows a trend of improved PC-ECLS-related bleeding rates and survival to hospital discharge over this period. 


\section{Introduction}

The prevalence of post-cardiotomy extracorporeal life support (PC-ECLS) reported in the literature varies between $0.6 \%$ and $2.6 \%$ of all cardiac surgical cases. ${ }^{1,2}$ Indications for PC-ECLS usually include intra-operative failure to wean from cardiopulmonary bypass due to perioperative left, right or biventricular failure, or refractory cardiogenic shock/cardiac arrest in the postoperative period. ${ }^{1-6}$ Recently, The Extracorporeal Life Support Organization (ELSO) Registry reported a remarkable increase in the use of ECLS as a supportive therapy after cardiac surgery. ${ }^{3,7-8}$ However a disappointing trend was observed with regards to the in-hospital survival rate, reaching only $15 \%$ in the latest period. ${ }^{3}$ Furthermore, despite this increase in use and new technological developments in this area, only a few comprehensive and dedicated reports about PC-ECLS have been presented. ${ }^{1,2,4,5}$ PC-ECLS, however, has represented the main indication for ECLS utilization ${ }^{7,8}$, and is characterized by specific aspects (duration and type of underlying disease, severity of comorbidities, indication and type of surgical procedure, modality of access and timing of implant, complication types and rates) when compared to other ECLS indications. Often these aspects are exacerbated when there is limited experience in these procedures, particularly in centres without well-established ECLS programmes. All of these factors may potentially influence ECLS management and final outcomes in postcardiotomy patients, and make this particular setting one in which there are higher risks for unfavourable results. ${ }^{3}$ Careful evaluation of patient features, complication rates and management, and in-hospital outcomes according to accumulated experience should provide meaningful information to assess centre performance. Furthermore, analysis of overall and trends of outcomes will hopefully indicate further areas of improvement to improve the ECLS results.

For these reasons, we reviewed the PC-ECLS series in our centre to elucidate patient characteristics and outcome throughout the last 10 years of experience. In doing so, we are able to highlight both positive and negative aspects, with the aim of indicating areas of strength or weakness of the overall PC-ECLS performance.

\section{Methods}


Patients from a single institution (Maastricht University Medical Centre [MUMC+], Maastricht, The Netherlands) who received ECLS, in the form of veno-arterial mode after cardiac surgery between October 2007 and June 2017 were included in this study. Data was retrospectively collected from hospital records including both surgery reports and intensive care unit data.

Inclusion criteria were age $\geq 18$ years old and cardiac surgery prior to ECLS. Indications for ECLS consisted of intra-operative failure to wean from cardiopulmonary bypass due to right, left or biventricular failure, and postoperative refractory cardiogenic shock or cardiac arrest during the hospitalization following the surgical procedure.

Patients with preoperative ECLS were excluded from the analysis.

The main outcome variables were survival to hospital discharge and postoperative bleeding rate. Statistical analysis.

In-hospital survivors and non-survivors were compared. The Shapiro Wilk test was used for normality testing of continuous variables. All variables were analysed with descriptive and frequency analysis. Chi-square Test and Fisher's Exact Test were used to compare group differences for categorical variables. Continuous variables were analysed by using the Mann Whitney U-test and were reported either as mean \pm standard deviation or as median \pm interquartile range. A p value $<$ 0.05 was considered a statistical significant difference between the groups. Variables that achieved a P-value of less than 0.2 in the univariate analysis were examined by using multivariate analysis with forward stepwise logistic regression to evaluate independent risk factors for the hospital mortality. The survival and bleeding rates were calculated, defining the respective trends over time. All statistical tests were performed with IBM® SPSS Statistics® software (Version 23.0. Armonk, NY: IBM Corp).

\section{Results}


During this 10-year period, 86 patients who were admitted to our hospital for cardiac surgery, received PC-ECLS. The mean age was 65 years (range 31-86 years, with 28 patients with age between 70 and 80 years, and 5 patients older than 80 years), and the majority of the subjects were male (65\%). All preoperative patient characteristics and details about cardiac disease are presented in Tables 1 and 2 , respectively.

\begin{tabular}{|c|c|c|c|c|}
\hline Variable & Total $(n-86)$ & In-hospital survivors ( $n-32$ ) & In-hospital non-survivors $(n-54)$ & $P(\text { exact, } 2 \text {-sided })^{*}$ \\
\hline Mean age, years (SD) & $65.0(11.2)$ & $62.6(12.5)$ & 66.5 (10.3) & 0.171 \\
\hline Femsle (\%) & $34.9 \%$ & $31.3 \%$ & $37.0 \%$ & 0.645 \\
\hline Mean BSA, $m^{2}(S D)$ & $1.91(0.18)$ & $1.91(0.19)$ & $1.92(0.18)$ & 0.963 \\
\hline Median BM, $\mathrm{kg} / \mathrm{m}^{2}$ (DQR) & $26.6(5.35)$ & $25.9(6.00)$ & $26.7(5.78)$ & 0.785 \\
\hline \multicolumn{5}{|l|}{ Medical history (\%) } \\
\hline Hypertension & $94.0 \%$ & $90.3 \%$ & $96.2 \%$ & 0.357 \\
\hline Smoking & $15.1 \%$ & $17.9 \%$ & $13.3 \%$ & 0.739 \\
\hline Hypercholesterolsemia & $96.3 \%$ & $93.5 \%$ & $98.0 \%$ & 0.554 \\
\hline Diabetes & $17.1 \%$ & $9.7 \%$ & $21.6 \%$ & 0.230 \\
\hline Diabetes with insulin use & $7.4 \%$ & $3.3 \%$ & $9.8 \%$ & 0.405 \\
\hline COPD & $12.5 \%$ & $6.7 \%$ & $16.0 \%$ & 0.306 \\
\hline Chronic renal failure & $4.9 \%$ & 0 & $7.8 \%$ & 0.291 \\
\hline Dialysis & $1.2 \%$ & 0 & $2.0 \%$ & 1.000 \\
\hline Stroke & $9.9 \%$ & $6.7 \%$ & $11.8 \%$ & 0.703 \\
\hline TIA & $14.8 \%$ & $25 \%$ & $13.3 \%$ & 0.345 \\
\hline Carotid stenosis & $3.8 \%$ & $6.7 \%$ & $2.0 \%$ & 0.553 \\
\hline Peripheral vascular disesse & $18.5 \%$ & $16.7 \%$ & $19.6 \%$ & 0.778 \\
\hline Pulmonary hypertension & $7.6 \%$ & $3.3 \%$ & $10.2 \%$ & 0.399 \\
\hline LVEF $<35 \%(\%)$ & $19.5 \%$ & $16.7 \%$ & $21.3 \%$ & 0.771 \\
\hline \multicolumn{5}{|l|}{ Preoperative condition (\%) } \\
\hline AF & $26.5 \%$ & $12.9 \%$ & $34.6 \%$ & 0.037 \\
\hline Pacemsiker $/ \mathrm{CD}$ & $9.6 \%$ & $9.7 \%$ & $9.6 \%$ & 1.000 \\
\hline Prior cardiac surgery & $20.9 \%$ & $21.9 \%$ & $20.4 \%$ & 1.000 \\
\hline AMI & $32.1 \%$ & $31.3 \%$ & $32.7 \%$ & 1.000 \\
\hline AMI $<30$ days & $19.0 \%$ & $21.3 \%$ & $17.3 \%$ & 0.776 \\
\hline $\mathrm{PCI}$ & $9.6 \%$ & $3.1 \%$ & $13.7 \%$ & 0.144 \\
\hline VSD & $2.4 \%$ & $3.1 \%$ & $1.9 \%$ & 1.000 \\
\hline Cardiogenic shock & $16.5 \%$ & $12.5 \%$ & $18.9 \%$ & 0.554 \\
\hline Cardisc arrest & $10.5 \%$ & $6.3 \%$ & $13.0 \%$ & 0.474 \\
\hline Pulmonary oedema & $9.5 \%$ & $3.1 \%$ & $13.5 \%$ & 0.147 \\
\hline RV dysiunction & $4.8 \%$ & $6.3 \%$ & $3.8 \%$ & 0.633 \\
\hline Vascopressors & $10.6 \%$ & $3.1 \%$ & $15.1 \%$ & 0.144 \\
\hline Intubation & $13.4 \%$ & $12.5 \%$ & $14.0 \%$ & 1.000 \\
\hline IABP & $10.5 \%$ & $15.6 \%$ & $7.4 \%$ & 0.283 \\
\hline Mean Euroscore $\|(\mathrm{QR})$ & $6.55(9.87)$ & $5.65(4.62)$ & $9.83(12.11\rangle$ & 0.111 \\
\hline
\end{tabular}




\begin{tabular}{|c|c|c|c|c|}
\hline Condition (\%) & Total $(n=86)$ & In-hospital survivors $(n=32)$ & In-hospital non-survivors $(n=54)$ & $P$ (exact, 2 -sided)* \\
\hline Coronary artery disease & $57.0 \%$ & $65.6 \%$ & $51.9 \%$ & 0.263 \\
\hline 3-vessel disease & $22.1 \%$ & $15.6 \%$ & $25.9 \%$ & 0.297 \\
\hline Aortic valve disease & $43.0 \%$ & $43.8 \%$ & $42.6 \%$ & 1.000 \\
\hline Mitral valve disease & $37.2 \%$ & $34.4 \%$ & $38.9 \%$ & 0.818 \\
\hline Tricuspid valve disease & $4.7 \%$ & $3.1 \%$ & $5.6 \%$ & 1.000 \\
\hline Thoracic aorta aneurysm & $10.5 \%$ & $3.1 \%$ & $14.8 \%$ & 0.145 \\
\hline Type-A dissection & $11.6 \%$ & $0 \%$ & $18.5 \%$ & 0.011 \\
\hline Type-B dissection & $1.2 \%$ & $3.1 \%$ & $0 \%$ & 0.372 \\
\hline Pulmonary embolism & $1.2 \%$ & $3.1 \%$ & $0 \%$ & 0.372 \\
\hline Bacterial endocarditis & $9.3 \%$ & $12.5 \%$ & $7.4 \%$ & 0.463 \\
\hline Cardiac tumour & $2.3 \%$ & $3.1 \%$ & $1.9 \%$ & 1.000 \\
\hline Other cardiac disease & $7.0 \%$ & $9.4 \%$ & $5.6 \%$ & 0.666 \\
\hline
\end{tabular}

The most common cardiac disease in this patient cohort was coronary artery disease, followed by aortic valve disease and mitral valve disease.

No differences between survivors and non-survivors were found in terms of preoperative comorbidities as well as in relation to preoperative left or right ventricular dysfunction, cardiogenic shock or cardiac arrest.

Type A aortic dissection was not considered a contraindication for PC-ECLS. However, no inhospital survivors were observed in this patient subgroup.

Operative procedures prior to ECLS are shown in Table 3. Previous cardiac surgery was reported in $20 \%$ of the patients, and most patients received elective surgery before ECLS cannulation. Only the combination of coronary artery bypass grafting with mitral valve surgery showed a statistical significant difference $(\mathrm{p}<0.033)$ with regards to in-hospital mortality. Cardiopulmonary bypass time was longer in non-survivors, whereas aortic cross-clamping time was lower compared to in-hospital survivors. Both variables, however, did not show any significant difference between groups. 


\begin{tabular}{|c|c|c|c|c|}
\hline Procedure & Total $(n=86)$ & $\begin{array}{c}\text { In-hospital } \\
\text { survivors }(n=32)\end{array}$ & $\begin{array}{c}\text { In-hospital } \\
\text { non-survivors }(n=54)\end{array}$ & $P$ (exact, 2-sided) ${ }^{*}$ \\
\hline Emergency surgery $(\%)$ & $38.4 \%$ & $34.4 \%$ & $40.7 \%$ & 0.649 \\
\hline Reoperation (\%) & $19.8 \%$ & $18.8 \%$ & $20.4 \%$ & 1.000 \\
\hline Off pump surgery (\%) & $3.5 \%$ & $6.3 \%$ & $1.9 \%$ & 0.553 \\
\hline Median aortic cross clamp time, minutes (IQR) & $104(90)$ & $108(97)$ & $98(92)$ & 0.754 \\
\hline Median CPB time, minutes (IQR) & $197(207)$ & $181(103)$ & $229(245)$ & 0.087 \\
\hline Normothermia (\%) & $34.9 \%$ & $34.4 \%$ & $35.2 \%$ & 1.000 \\
\hline CABG (\%) & $22.1 \%$ & $25.0 \%$ & $20.4 \%$ & 0.789 \\
\hline AV surgery (\%) & $8.1 \%$ & $12.5 \%$ & $5.6 \%$ & 0.416 \\
\hline MV surgery (\%) & $4.7 \%$ & $0 \%$ & $7.4 \%$ & 0.292 \\
\hline Thoracic aorta surgery (\%) & $3.5 \%$ & $0 \%$ & $5.6 \%$ & 0.291 \\
\hline CABG + AV surgery $(\%)$ & $9.3 \%$ & $15.6 \%$ & $5.6 \%$ & 0.142 \\
\hline CABG + MV surgery (\%) & $11.6 \%$ & $21.9 \%$ & $5.6 \%$ & 0.033 \\
\hline CABG + thoracic aorta surgery $(\%)$ & $1.2 \%$ & $0 \%$ & $1.9 \%$ & 1.000 \\
\hline$A V+M V$ surgery $(\%)$ & $3.5 \%$ & $6.3 \%$ & $1.9 \%$ & 0.553 \\
\hline$A V+$ thoracic aorta surgery (\%) & $9.3 \%$ & $3.1 \%$ & $13.0 \%$ & 0.249 \\
\hline$M V+$ thoracic aorta surgery (\%) & $2.3 \%$ & 0 & $3.7 \%$ & 0.527 \\
\hline Other surgery (\%) & $24.4 \%$ & $15.6 \%$ & $29.6 \%$ & 0.196 \\
\hline
\end{tabular}

ECLS implant and weaning details are shown in Table 4. The most frequent indication for ECLS in this cohort was failure to wean from cardiopulmonary bypass. Indeed, the majority of the ECLS runs were commenced in the operating room directly after surgery, with the remaining cases started in the intensive care unit. Peripheral cannulation represented the majority of ECLS catheter access, followed by similar rates between central or combination of central arterial and peripheral venous cannulation. Cannulation strategy did not appear to influence survival in this particular patient cohort, as summarized in Table 4. The additional support to ECLS with intra-aortic balloon pump (IABP) was used in $25 \%$ of the patients to favour left ventricular unloading and opening of the aortic valve, as well as to increase coronary flow.

Patients were kept on ECLS for a median duration of 5.0 days (IQR 6). For in-hospital survivors this was 6 days (IQR 5) and for in-hospital non-survivors this was 4 days (IQR 6). This difference proved not to be statistically significant. 


\begin{tabular}{|c|c|c|c|c|}
\hline Procedure & Total $(n=86)$ & $\begin{array}{c}\text { In-hospital } \\
\text { survivors }(n=2)\end{array}$ & $\begin{array}{c}\text { In-hospital } \\
\text { non-survivors }(n=54)\end{array}$ & $P$ (exact, 2 -sided) ${ }^{*}$ \\
\hline Weaning CPB with ECLS (\%) & $53.5 \%$ & $46.9 \%$ & $57.4 \%$ & 0.378 \\
\hline Weaning CPB with $\mathrm{LABP}(\%)$ & $9.3 \%$ & $12.5 \%$ & $7.4 \%$ & 0.463 \\
\hline ECLS placed during OR (\%) & $55.8 \%$ & $50.0 \%$ & $59.3 \%$ & 0.501 \\
\hline ECLS placed in ICU (\%) & $44.2 \%$ & $50.0 \%$ & $40.7 \%$ & 0.501 \\
\hline ECLS implanted central (\%) & $17.4 \%$ & $15.6 \%$ & $18.5 \%$ & 0.779 \\
\hline ECLS implanted peripheral (\%) & $65.1 \%$ & $65.6 \%$ & $64.8 \%$ & 1.000 \\
\hline ECLS implanted arterial central, venous peripheral (\%) & $17.4 \%$ & $18.8 \%$ & $16.7 \%$ & 1.000 \\
\hline ECLS indication, failure to wean $(\%)$ & $52.3 \%$ & $43.8 \%$ & $57.4 \%$ & 0.267 \\
\hline ECLS indication, post-cardiotorny cardiogenic shock (\%) & $47.7 \%$ & $56.3 \%$ & $42.6 \%$ & 0.267 \\
\hline Resuscitation between CPB and ECLS implantation (\%) & $24.4 \%$ & $31.3 \%$ & $20.4 \%$ & 0.304 \\
\hline Perioperative low cardiac output syndrome $(\%)$ & $50.0 \%$ & $43.8 \%$ & $53.7 \%$ & 0.504 \\
\hline Post-operative IABP (\%) & $27.1 \%$ & $32.3 \%$ & $24.1 \%$ & 0.454 \\
\hline Median ECLS duration, days (QRR) & $5.0(6.0)$ & $6.0(5.0)$ & $4.0(6.0)$ & 0.219 \\
\hline
\end{tabular}

The use of PC-ECLS was associated with several complications (Table 5). Post-operative cardiac arrhythmias and bleeding, followed by acute renal injury, right ventricular dysfunction, sepsis, and major neurological impairment were the most common complications. Interestingly, atrial fibrillation was more common in the group of in-hospital survivors, whilst ventricular fibrillation was seen more often in the group of in-hospital non-survivors. Re-thoracotomy was performed in the majority of the patients, mostly due to bleeding complications and suspicion of cardiac tamponade.

\begin{tabular}{|c|c|c|c|c|}
\hline Variable & Total $(n-86)$ & In-hospital survivors ( $n-32$ ) & In-hospital non-survivors $(n-54)$ & $P(\text { exact, } 2 \text {-sided })^{*}$ \\
\hline Arrhytheria & $47.1 \%$ & $65.6 \%$ & $35.8 \%$ & 0.009 \\
\hline AF & $20.0 \%$ & $37.5 \%$ & $9.4 \%$ & 0.003 \\
\hline vT & $7.1 \%$ & $6.3 \%$ & $7.5 \%$ & 1.000 \\
\hline VF & $17.6 \%$ & $12.5 \%$ & $20.8 \%$ & 0.392 \\
\hline Other & $9.4 \%$ & $15.6 \%$ & $5.7 \%$ & 0.146 \\
\hline Pacemaker/CD & $3.6 \%$ & $9.7 \%$ & 0 & 0.047 \\
\hline Re-thoracotorry for bleeding & $45.4 \%$ & $50.0 \%$ & $44.2 \%$ & 0.657 \\
\hline Acute kidney injury & $29.8 \%$ & $18.8 \%$ & $36.5 \%$ & 0.093 \\
\hline Respiratory insufficiency & $14.5 \%$ & $12.9 \%$ & $15.4 \%$ & 1.000 \\
\hline TIA & 0 & 0 & 0 & na \\
\hline Stroke & $11.9 \%$ & $18.8 \%$ & $7.7 \%$ & 0.170 \\
\hline Cerebral hoemorrhage & 0 & 0 & 0 & na \\
\hline Major neurological impsirment & nt $20.2 \%$ & $15.6 \%$ & $23.1 \%$ & 0.577 \\
\hline Brain death & $9.5 \%$ & 0 & $15.4 \%$ & 0.022 \\
\hline Sepsis & $21.4 \%$ & $18.8 \%$ & $23.1 \%$ & 0.786 \\
\hline Vasoplegic syndrome & $13.1 \%$ & $3.1 \%$ & $19.2 \%$ & 0.045 \\
\hline RV dysfunction & $29.8 \%$ & $36.5 \%$ & $18.8 \%$ & 0.093 \\
\hline Leg ischaemia & $10.7 \%$ & $18.8 \%$ & $5.8 \%$ & 0.078 \\
\hline Leg fasciotorny & $4.8 \%$ & $9.4 \%$ & $1.9 \%$ & 0.152 \\
\hline Gl complications & $14.3 \%$ & $9.4 \%$ & $17.3 \%$ & 0.359 \\
\hline Bowel ischaernia & $1.2 \%$ & $0 \%$ & $1.9 \%$ & 1.000 \\
\hline Laparotorny required & $4.8 \%$ & $3.1 \%$ & $5.8 \%$ & 1.000 \\
\hline Pneumonia & $10.7 \%$ & $12.5 \%$ & $9.6 \%$ & 0.726 \\
\hline ARDS & 0 & 0 & 0 & ns \\
\hline
\end{tabular}


Interestingly, the bleeding and re-thoracotomy decreased in the last two years (Figure 1).

\section{Figure 1. Rates of re-thoracotomy for bleeding during the study period in patients receiving a post-cardiotomy extracorporeal life support implant}

\section{Post Operative Bleeding}

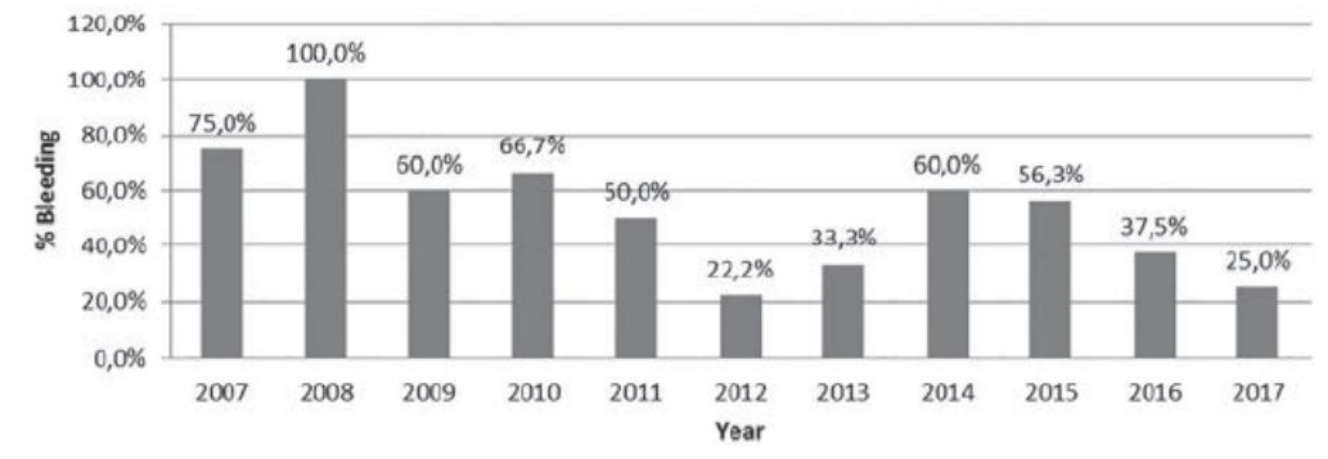

Major neurological impairment was observed in $20 \%$ of cases. Postoperative vasoplegic syndrome was a frequent event with a significant difference between survivors and non-survivors (Table 5).

Overall, successful ECMO weaning was possible in $49,4 \%$ of the cases, whereas survival to hospital discharge was achieved in $37 \%$ of the cases. The main reason for death was a cardiac event, followed by multi-organ failure and neurologic complications. A positive trend towards improved in-hospital survival was observed over the course of the study period (Figure 2). 


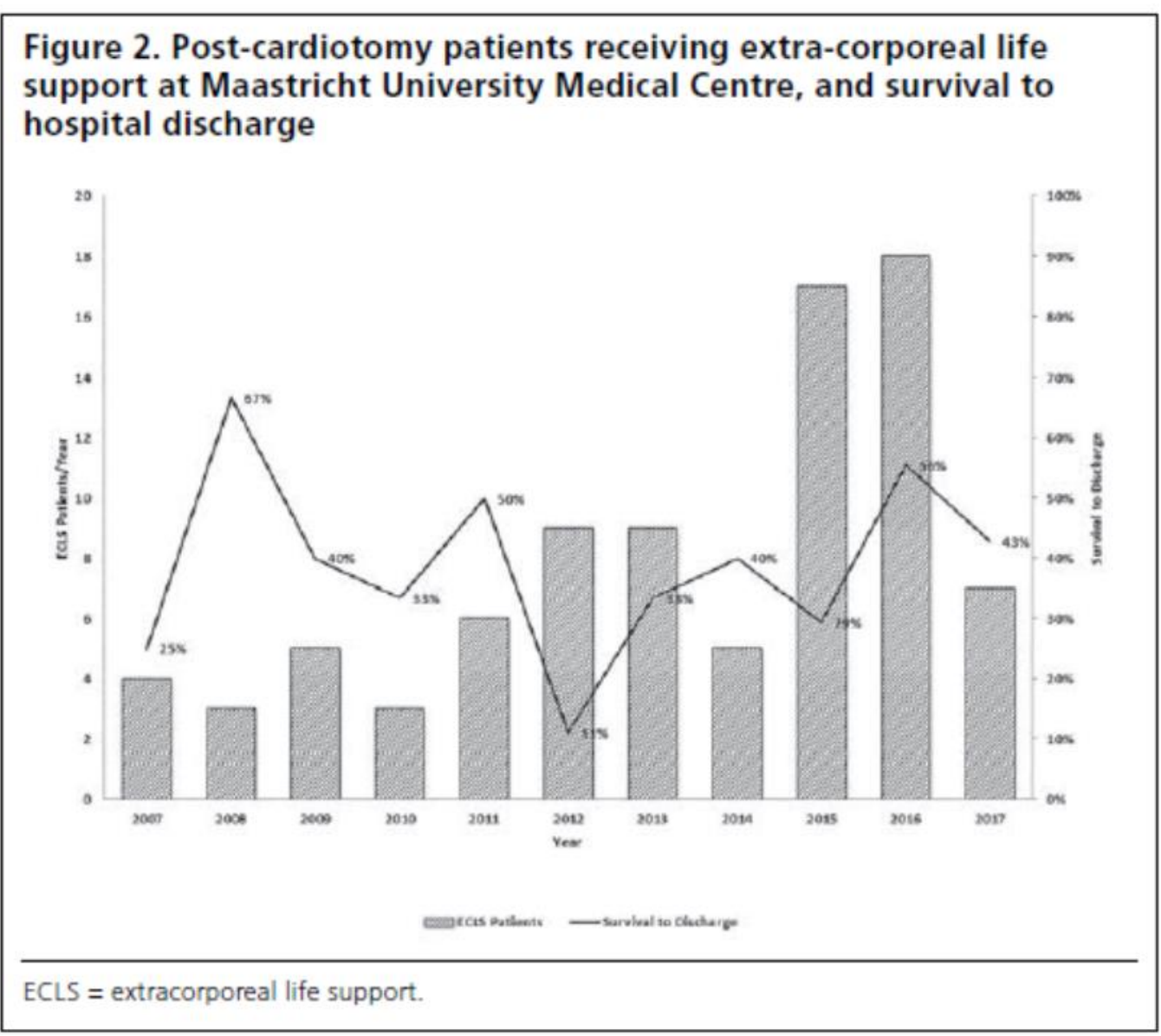

At multiple logistic regression analysis, age $>65$ years and postoperative arrhythmia were independent risk factor for mortality (Table 6).

\begin{tabular}{|c|c|c|c|}
\hline Variable & OR & SE & $P$ \\
\hline Postoperative arrhythmia & 0.283 & 0.576 & 0.028 \\
\hline Age $>65$ years & 4.348 & 0.614 & 0.017 \\
\hline \multicolumn{4}{|c|}{$\begin{array}{l}\text { OR = odds ratio. SE = standard error. * The following variables were } \\
\text { included in the analysis: pre-operative atrial fibrillation, pre-operative } \\
\text { percutaneous coronary intervention, acute pulmonary oedema, } \\
\text { pre-operative vasopressors, pre-operative EURO-score, type-A aortic } \\
\text { dissection, cardiopulmonary bypass time, aortic cross-clamping time, } \\
\text { pre-operative arrhythmia, post-operative vasoplegic syndrome, post- } \\
\text { operative right ventricular dysfunction, post-operative left ventricular } \\
\text { dysfunction, post-operative leg ischaemia, combo-operation (coronary } \\
\text { artery bypass grafting plus aortic valve/mitral valve/thoracic aorta or } \\
\text { aortic valve plus mitral valve or thoracic aortic plus mitral valve), } \\
\text { age }>65 \text { years. }\end{array}$} \\
\hline
\end{tabular}

\section{Discussion}

Our 10-year experience shows that 1) PC-ECLS use is increasing and can be widely used in all postcardiotomy scenarios; 2) the results of this temporary support can be improved with increasing case 
volume and centre expertise, as well as with better control of PC-ECLS related complications and management; 3) underlying cardiac disease and the corresponding cardiac procedure as well emergency, reoperation, endocarditis, do not influence the hospital mortality, except from ECLS in acute aortic dissection; 4) the ECLS configuration (central vs. peripheral cannulation) and the use of IABP do not influence survival; 5) PC-ECLS is still accompanied by a high mortality, though with an acceptable weaning rate (nearly 50\%) in patients with an otherwise unfavorable prognosis.

Post-cardiotomy shock is a catastrophic entity. The reported definition includes the inability to wean from cardiopulmonary bypass, requiring mechanical circulatory support system; the need for an mechanical circulatory support within 48 hours or earlier after the index procedure; and the use of a mechanical circulatory support at any point during the initial hospital stay. ${ }^{6}$ Patients suffering from post-cardiotomy shock are typically characterized by high preoperative creatinine blood levels, previous myocardial infarction $\mathrm{MH}$, presence of left-main disease, left ventricle (LV) dysfunction, prolonged history of coronary artery disease, redo operations, recent operation, unstable clinical status, and emergency. ${ }^{4}$

Trend and mortality. PC-ECLS has been shown to be the most frequent indication for catheter implantation in the United States until $2011 .^{7}$ In a retrospective analysis of ECLS as therapy for postcardiotomy shock in the United States between 2002 and 2012, McCarthy and colleagues ${ }^{8}$ found a significant increase in ECLS application in this setting. The reported mortality of $60 \%$ in the study is the highest mortality for all ECMO indications reported. These results have been also confirmed in a 15-year analysis of the ELSO database. ${ }^{3}$ This report has shown that although ECLS use has increased exponentially during the time, it appears that survival rates to discharge have, if anything, decreased. There are a number of potential explanations for this observation, including the learning curve of the centres using this treatment, to more complex patient profiles, or even to the more liberal use of PCECLS and this technique often being applied as a "last resort" in moribund patients.

Our results showed an overall survival comparable to those reported in the literature. ${ }^{1,3,6,8}$ However, we observed a marked improvement in survival in the most recent years, most likely reflecting the 
increase in volume and centre experience, as well as a better complication prevention and management.

Technical considerations. There is still much debate with regards to which cannulation site (central versus peripheral) should be utilised to achieve the best recovery and an easier management. ${ }^{9}$ In the largest single-center report, Rastan and colleagues ${ }^{2}$ reported on 517 patients $(1.2 \%$ of the total cardiac surgery cases) who required ECLS for post-cardiotomy shock. In this study, no advantages were observed by using a central arterial cannulation site relative to a peripheral site or to an axillary artery approach relative to a femoral artery approach. In fact, percutaneous femoral vein drainage was associated with a worse prognosis, and may be a surrogate of suboptimal venous drainage and compromised ECLS flow. By contrast, Loforte ${ }^{10}$ showed that central cannulation in PC-ECLS had increased bleeding and continuous veno-venous hemofiltration rates compared with peripheral access (62.7\% versus $48.4 \%$, and $56.8 \%$ versus $43.6 \%$, respectively).

During the central configuration of ECLS, LV venting, either directly or indirectly, is usually more frequently adopted, but its role and potentially benefit are still controversial. ${ }^{11}$ In our series, application of LV unloading, has been more frequently and carefully addressed and possibly plays an additional role in enhanced ECLS benefit and outcome. The role of IABP placement in aiding recovery is also controversial. The beneficial effects of adding IABP support to ECLS on coronary perfusion, and on reduction of the LV afterload have been well demonstrated in animal models ${ }^{13}$, and single-centre experiences have shown the efficacy in terms of outcome compared with no IABP use. ${ }^{14}$ Nevertheless, some have abandoned the use of IABP in combination with ECLS because of the increased risk of vascular complications. ${ }^{15}$ Further studies are therefore warranted in this respect to ascertain advantages and to elucidate mode, effects, and results of LV unloading during ECLS.

Changes of ECLS configuration during the temporary support has been recently highlighted as an additional possibility and need during the ECLS run. Commencing with conventional veno-arterial ECLS with a single venous and arterial cannulation, either centrally or peripherally, may be insufficient or not ideal for the ongoing changes of pathophysiological and hemodynamic conditions 
and needs. The addition of another cannula, either for venous return or oxygenation, or venting, represent potential beneficial scenarios.

Complications in patients on ECLS are frequent and strongly affect the final outcome. ${ }^{2,3}$ ECLS duration varies in published literature on PC-ECLS, and though this does not seem to impact the weaning rate, a shorter duration of ECLS has been linked to better survival rates, mainly due to reduction of complication rates. ${ }^{2,12}$ Bleeding and re-thoracotomy, gastrointestinal bleeding, limb ischemia, and neurological impairment are well reported complications of ECLS, and our experience is consistent with the literature. Bleeding is a major problem in postoperative ECLS care, with rates as high as $90 \% .^{16,17}$

Reasons for excessive bleeding in these patients include surgical trauma, thrombocytopenia, activation of leucocytes, and anticoagulation treatment. In our experience, proper hemostasis of the patients supported with ECMO after cardiac surgery with closed chest if feasible, represent our main goals in PC-ECLS patients. Protamine administration, sternal closure, and delayed anticoagulation treatment (at least 24 hours or later) are highly recommended strategies to reduce the bleeding risk. In the presence of peripheral vascular disease, a switch from central to peripheral ECMO with heparin coated circuits may also be applied to reduce the incidence of limb ischemia and related complications. However, the use of distal perfusion of the cannulated limb for arterial perfusion represents the critical action to reduce or prevent such an adverse event.

Our data with regards to other complication types and rates are in accordance to published literature. ${ }^{2-}$ ${ }^{4}$ Of note, data from the ELSO Registry, about patients submitted to veno-arterial ECLS, has shown that neurologic complications occur in $15 \%$ of the cases, leading to almost $90 \%$ mortality. ${ }^{18}$ This rate may be slightly higher in PC-ECLS patients, as shown in our and other investigators' findings. ${ }^{2,4}$ Currently, however, there is no consistent action recommended or advisable to reduce such adverse events. It is hoped that additional refinement in ECLS technology and anticoagulation management will impact the mechanisms underlying cerebral insult and injury and lead to improvements in this area. 
Age is a well-known risk factor for hospital mortality in post cardiotomy ECLS support ${ }^{19}$ and our findings are consistent with that. Recently, the influence of age in ECLS was investigated from the ELSO. ${ }^{20}$ More than 700 patients older than 70 years were analysed and the promising $30.5 \%$ of survival to hospital discharge encourages further studies in this field.

Based on the above-mentioned factors, we have recently adopted a "patient-tailored concept" of ECLS configuration and management in post-cardiotomy, which appears to provide actual benefit on patient outcome and trends: 1) ECLS mode must account for patient's preoperative status (function of right and left ventricle), underling disease (chronic versus acute) and intra-operative status and requirement, avoiding a repetitive and fixed ECLS configuration; 2) Sternal closure should be addressed as often as possible, also in the central configuration, to reduce perioperative bleeding. 3 ) Prevention or reduction of LV distension through a cannulation of pulmonary artery used as additional drainage or using other LV venting techniques and devices; 3) IABP is highly recommended in order to favour LV unloading and opening of the aortic valve thereby enhancing LV unloading and reducing the risk of left cardiac chamber thrombosis; 4) Use of distal limb perfusion in case of femoral artery cannulation in order to reduce limb ischemia, and a sheathless IABP implant; 5) the use of new design of IABP in the shorter configuration is also recommended to reduce the risk of mesenteric and renal impairment. ${ }^{13}$

\section{Study Limitations.}

This is a single-centre series of consecutive patients, which have been retrospectively reviewed. In addition, although there was no difference in age between survivors and non survivors, 19 deaths occurred in patients between 70 and 80 years and 3 in those $>80$ years. The true influence of age on post- PC-ECLS events warrants further investigation. Furthermore, the study population showed a heterogeneity in procedures and cannulation strategy and management. Finally, in a number of cases data is missing (e.g., hemodynamic and labs parameters) and therefore poses an additional limitation.

\section{Conclusion.}


ECLS use after cardiac surgery is increasing, but is still limited by high mortality rates and resource consumption despite all efforts and advancements in technology. Our experience confirms the data already reported, but highlight that careful patient management, and increased expertise may lead to a reduction of complication rate and improved results.

Alternative configurations and cannulation strategies tailored specifically to the status of patient, as well as a dedicated program, increased expertise, and a multidisciplinary approach can improve knowledge in the field and hopefully ultimately benefit patients undergoing PC-ECLS. 


\section{References}

1. Elsharkawy HA, Li L, Esa WA, et al. Outcome in patients who require venoarterial extracorporeal membrane oxygenation support after cardiac surgery. J Cardiothorac Vasc Anesth. 2010; 24: 946-51.

2. Rastan AJ, Dege A, Mohr M, et al. Early and late outcome of 517 consecutive patients treated with extracorporeal membrane oxygenation for refractory postcardiotomy cardiogenic shock. J Thorac Cardiovasc Surg. 2010; 139: 302-11.

3. Whitman GJ. Extracorporeal membrane oxygenation for the treatment of postcardiotomy shock. J Thorac Cardiovasc Surg. 2017; 153: 95-101.

4. Smedira NG, Blackstone EH. Postcardiotomy mechanical support: risk factors and outcome. Ann Thorac Surg. 2001; 71: 60-66.

5. Saxena P, Neal J, Joyce LD, et al. Extra-corporeal membrane oxygenation support in postcardiotomy elderly patients: the Mayo Clinic experience. Ann Thorac Surg. 2015; 99: 2053-2060. 6. Fukuhara S, Takeda K, Garan AR, et al. Contemporary mechanical circulatory support therapy for postcardiotomy shock Gen Thorac Cardiovasc Surg. 2016; 64: 183-19.

7. Sauer CM, Yuh DD, Bonde P. Extracorporeal membrane oxygenation use increased $433 \%$ in adults in the United States from 2006 to 2011. ASAIO J 2015; 61: 31-6.

8. McCarthy FH, McDermott KM, Kini V, et al. Trends in U.S. Extracorporeal Membrane Oxygenation Use and Outcomes: 2002-2012. Semin Thorac Cardiovasc Surg. 2015; 27: 81-8.

9. Storey, J. D. and R. Tibshirani (2003). "Statistical significance for genomewide studies. Proceedings of the National Academy of Sciences 100(16): 9440-9445

10. Sorokin V, MacLaren G, Vidanapathirana PC, et al. Choosing the appropriate configuration and cannulation strategies for extracorporeal membrane oxygenation: the potential dynamic process of organ support and importance of hybrid modes. Eur J Heart Fail. 2017; 19: 75-83.

11. Loforte A, Marinelli G, Musumeci F, et al. Extracorporeal membrane oxygenation support in refractory cardiogenic shock: treatment strategies and analysis of risk factors. Artif Organs 2014; 38 : $129-41$. 
12. Meani P, Gelsomino S, Natour E, et al. Modalities and Effects of Left Ventricle Unloading on Extracorporeal Life support: a Review of the Current Literature. Eur J Heart Fail. 2017; 19: 84-91.

13. Ko WJ, Lin CY, Chen RJ, et al. Extracorporeal membrane oxygenation support for adult postcardiotomy cardiogenic shock. Ann Thorac Surg 2002; 73: 538-45

14. Gelsomino S, Lozekoot PW, Lorusso R, et al. Comparing short versus standard-length balloon for intra-aortic counterpulsation: results from a porcine model of myocardial ischaemia-reperfusion. Eur J Cardiothorac Surg. 2016; 49: 1361-9.

15. Doll N, Kiaii B, Borger M, et al. Five-year results of 219 consecutive patients treated with extracorporeal membrane oxygenation for refractory postoperative cardiogenic shock. Ann Thorac Surg. 2004; 77:151-157.

16. Marasco SF, Vale M, Pellegrino V, et al. Extracorporeal membrane oxygenation in primary graft failure after heart transplantation. Ann Thorac Surg. 2010; 90:1541-46.

17. Lazzara RR, Magovern JA, Benckart DH, et al. Extracorporeal membrane oxygenation for adult post-cardiotomy cardiogenic shock using heparin bonded system. ASAIO J 1993; 39: M444-M447.

18. Bakhtiary F, Keller H, Dogan S, et al. Venoarterial extracorporeal membrane oxygenation for treatment of cardiogenic shock: clinical experiences in 45 adult patients. J Thorac Cardiovasc Surg $2008 ; 135: 382-8$.

19. Lorusso R, Barili F, Di Mauro M, et al In-hospital neurologic complications in adult patients undergoing venoarterial extracorporeal membrane oxygenation: results from the Extracorproeal Life Support Organization (ELSO). Crit Care Med 2016; 44: 964-72.

20. Lorusso R, Gelsomino S, Parise O, et al. Veno-Arterial Extracorporeal Membrane Oxygenation for Refractory Cardiogenic Shock in Elderly Patients: Trends in Application and Outcome from the Extracorporeal Life Support Organization (ELSO) Registry. Ann Thorac Surg 2017; 104: 62-69. 


\title{
CHAPTER 4
}

\author{
Meta-Analysis of Peripheral or Central ECMO \\ in Postcardiotomy and Non-Postcardiotomy Shock
}

Raffa GM, Kowalewski M, Brodie D, Ogino M, Whitman G, Meani P, Pilato M, Arcadipane A, Delnoij T, Natour E, Gelsomino S, Maessen J, Lorusso R.

The Annals of Thoracic Surgery. 2019 Jan;107(1):311-321. 


\section{Abstract}

Background. Veno-arterial extracorporeal membrane oxygenation (VA-ECMO) application in postcardiotomy shock (PCS) and non-PCS is increasing. VA-ECMO plays a critical role in the management of these patients, yet may be associated with serious complications.

Methods. A systematic review of all available reports in the literature of patients receiving VAECMO, either directly or indirectly comparing central cannulation (right atrial to ascending aorta) versus peripheral cannulation (femoral vein to femoral artery or axillary artery) were analyzed. The primary endpoint was survival. Cerebrovascular events, limb complications, bleeding requiring reoperation, sepsis, continuous veno-venous hemofiltration, and transfusions, were also assessed in both groups.

Results. Seventeen retrospective case series clearly describing the VA-ECMO access and including 1,691 patients with PC- and non-PCS were found. The peripheral approach was more commonly used (980 patients, $57.9 \%$ ) than the central one. There was no difference in the analysis between the two techniques regarding all-cause mortality RR (Risk Ratio) 95 CIs (Confidence Intervals): 1.00 (0.941.08) $I^{2}=0 \%, \mathrm{P}=0.92$. No statistical differences were found between peripheral and central VAECMO with regards to cerebrovascular events, limb complications, or sepsis rates. Peripheral cannulation, was associated with a significant reduction in the risk of bleeding $(\mathrm{p}=0.02)$, continuous veno-venous hemofiltration $(\mathrm{p}=0.03)$, transfusion of red blood cells units $(\mathrm{p}<0.00001)$, fresh frozen plasma units $(\mathrm{p}=0.0002)$ and platelets units $(\mathrm{p}<0.00001)$.

Conclusions. Peripheral and central VA-ECMO configurations showed comparable in-hospital survival in PCS and non-PCS. The risk of bleeding, continuous veno-venous hemofiltration and blood product transfusion was significantly lower with the peripheral cannulation strategy. 


\section{Introduction}

The application of veno-arterial (VA) extracorporeal membrane oxygenation (ECMO) in patients with refractory isolated cardiac or cardio-pulmonary failure, is increasing [1]. In adult patients, "central" access, with the direct cannulation of the right atrium and ascending aorta, and "peripheral" access, most commonly using the femoral vein and artery, have been the predominant modalities for the initiation of VA-ECMO. Alternative approaches have included the use of a vascular prostheses for the thoracic aorta as central access, or the axillary, subclavian, or innominate arteries, either through a direct cannulation or with the interposition of a vascular graft. Undoubtedly, the application of VA-ECMO during cardiac arrest or in non-postcardiotomy shock (PCS) represent the typical scenarios in which peripheral cannulation is commonly adopted because it is a faster and easier strategy, whereas central access plays a larger role in PCS [2].

The optimal cannulation strategy for VA-ECMO, in terms of survival as well as myocardial recovery, management and complication rate, remains controversial [3]. Despite the considerable numbers of publication regarding VA-ECMO, only a few have addressed access-related issues [4-18], particularly as the primary focus of the studies $[7,8,10]$.

In the largest single center series, Rastan and colleagues [4] reported no advantage in survival with the use of different cannulation sites in 517 patients who required VA-ECMO after cardiac surgery. Although there has been a general consensus favoring a peripheral approach in PCS and non-PCS [49], a recent study demonstrated that a central approach should be considered a viable alternative in terms of complications rate [10]. Based on the above controversies, we performed a systematic review and meta-analysis of studies reporting the in-hospital outcomes of central versus peripheral VAECMO in PCS and non-PCS settings.

\section{Material and Methods}

\section{Definition}


Central cannulation was defined as cannulation involving the aorta for the patients' arterial inflow and right atrium or both venae cave for patients' outflow. Peripheral cannulation was defined as cannulation of the femoral and axillary artery for patients' arterial inflow and femoral vein for patients' inflow. Right atrium and femoral artery cannulation or aorta and femoral vein cannulation strategies were considered peripheral and central, respectively, since the access for inflow cannula dictates the actual configuration.

Bleeding was defined as any bleeding requiring reoperation. Limb complication was defined as any limb complication involving the vascular access (excluding groin and wound infection)

Distal limb perfusion strategy in order to avoid limb ischemia in case of peripheral cannulation included the cannulation of a dacron or hemashield prosthetic graft anastomosed end to side onto the femoral artery thus maintaining arterial flow to the ipsilateral lower limb or the insertion of a distal perfusion cannula inserted into the femoral artery.

\section{Data sources and search strategy}

This systematic review and meta-analysis was performed in accordance with the Preferred Reporting Items for Systematic Reviews and meta-analyses (PRISMA) statement [19]. The PRISMA checklist is available as Appendix Table 1. Relevant studies to be included were searched for through October $31^{\text {st }}$ 2017, through PubMed, EMBASE, CINAHL, the Web of Science, the Cochrane Register of Controlled Clinical Trials (CENTRAL) and Google Scholar, as well as congress proceedings from major cardiothoracic and cardiology societies meetings. The search term was: "extracorporeal membrane oxygenation". The literature was limited to articles published in English. References of original articles were reviewed manually and cross-checked for other relevant reports.

\section{Selection criteria, quality assessment and outcomes}

Studies were included if they met all of the following criteria: 1) human study; 2) studies comparing directly peripheral versus central cannulation in patients undergoing VA-ECMO for cardiogenic shock; 3) studies reporting on VA-ECMO outcomes of interest with separate (indirect comparison) results for peripheral versus central cannulation. Exclusion criteria were as follows: 1) paediatric and 
congenital heart surgery-related studies; 2) animal studies; 3) studies not reporting cannulation site strategy for VA-ECMO. Reviews were not considered.

Two independent reviewers (G.M.R and M.K.) selected the studies for inclusion, extracted studies, as well as patient characteristics of interest and relevant outcomes. Two authors (G.M.R. and M.K.) independently assessed the trials' eligibility and risk of bias. Risk of bias at the individual study level was assessed using the ROBINS-I tool (Risk of Bias in Not-randomized Studies-of Interventions) [20]. Any divergences were resolved by a third reviewer (R.L.) and quantified using the approach of Cohen's kappa.

\section{Endpoint selection}

The primary endpoint was in-hospital mortality. Secondary endpoints were in-hospital cerebrovascular events (CVE), limb complications, bleeding requiring reoperation, sepsis, continuous veno-venous hemofiltration $(\mathrm{CVVH})$ and transfusions. Long-term follow-up and out-of-hospital data were not considered.

\section{Statistical analysis}

Data were analysed according to the intention-to-treat principle wherever applicable. Risks Ratios (RRs) and 95\% Confidence Intervals (CIs) served as primary index statistics for dichotomous outcomes; for continuous outcomes, mean difference and corresponding 95\% CIs were calculated using a random effects model. To overcome the low statistical power of the Cochran Q test, the statistical inconsistency test $I^{2}=\left[\left(\mathrm{Q}_{-} d f\right) / \mathrm{Q}\right]$ x $100 \%$, where $\mathrm{Q}$ is the chi-square statistic and $d f$ its degrees of freedom, was used to assess heterogeneity [21]. It examines the percentage of inter-study variation, with values ranging from $0 \%$ to $100 \%$. An $I^{2}$ value of less than $40 \%$ indicates no obvious heterogeneity, values between $40 \%-70 \%$ suggest moderate heterogeneity and $I^{2}>70 \%$ were considered high heterogeneity.

Because of the high degree of heterogeneity anticipated among the available studies (only nonrandomized trials) an inverse variance (DerSimonian-Laird) random-effects model was applied as a 
more conservative approach for observational data accounting for between- and within-study variability. Whenever a single study reported median values and interquartile ranges instead of mean $\pm \mathrm{SD}$, the latter were approximated as described by Wan and colleagues [22]. Potential publication bias was evaluated for the primary endpoint by constructing a "funnel plot" in which the standard error of the log RR was plotted against the RR. The asymmetry of the plot was estimated both visually and by a linear regression approach. Pre-specified sensitivity analysis for the primary endpoint was conducted and stratified by type of heart surgery where applicable: 1) post coronary artery bypass grafting $(\mathrm{CABG}) ; 2$ ) post valvular surgery and 3) combined or other survery. The latter group encompassed combined coronary and valvular surgery, along with surgery on thoracic aorta, other cardiac surgery and non-PCS indications for VA-ECMO. Additionally, by means of metaregression, the impact of percentage prevalence of non-PCS patients across single studies and its relationship to occurrence of the primary endpoint was investigated. Review Manager V.5.3 (The Nordic Cochrane Centre, Copenhagen, Denmark) and Comprehensive Meta-Analysis, v. 2 (Biostat, Englewood Cliffs, NJ) were used for statistical computations. $P$-values $<0.05$ were considered statistically significant and reported as two-sided, without adjustment for multiple comparisons.

\section{Results}

The PRISMA flow diagram describing the study selection process along with reasons for exclusion is presented in Appendix Figure 1. After removal of reports not pertinent to the design of the current meta-analysis, 17 retrospective observational studies that met explicit inclusion criteria remained, including a total of 1,691 patients. Of those, 980 patients $(57.95 \%)$ underwent peripheral, and 711 $(42.05 \%)$ central cannulation for VA-ECMO. Most commonly (85.1\% of the time), VA-ECMO was instituted for PCS; remaining indications included: acute myocardial infarction (5.6\%), decompensated cardiomyopathy $(5.0 \%)$, myocarditis $(0.8 \%)$ and other non post-surgical indication (3.5\%). From a surgical standpoint, the majority of ECMOs followed coronary artery bypass grafting (33.9\%), valve surgery (15.4\%) and mixed cases (8.7\%). VA-ECMO followed aortic surgery in 103 
cases $(6.1 \%), 36$ of which (2.1\%) were for acute type A aortic dissection. Patients undergoing VAECMO had a mean age of 61.7 years and $68 \%$ were male. Baseline EuroSCOREs ranged between $6.2 \%$ up to $25.7 \%$. Follow-up reporting across the studies varied between 30 -day and in-hospital survival. Detailed characteristics of studies and patients are listed in Table 1. 
Table 1. Studies, Periphoal Extracorpareal Monbrate Oxygotation Venas Cottral Extradrpor aul Mombrate Orygenation, and Patiens' Basdine Ohanactristics

Study First Author, Year [Reference]

\begin{tabular}{|c|c|c|c|c|c|c|c|c|c|}
\hline Variables & $\begin{array}{l}\text { Ranney, } \\
2017 \text { [10] }\end{array}$ & $\begin{array}{l}\text { Blancari, } \\
2017 \text { [12] }\end{array}$ & $\begin{array}{l}\text { Guilhaire, } \\
2017 \text { [17] }\end{array}$ & $\begin{array}{l}\text { Slotbsch } \\
2017 \text { [15] }\end{array}$ & $\begin{array}{c}\text { Raffa, } \\
2017 \text { [4] }\end{array}$ & $\begin{array}{c}\text { Khorsund, } \\
2016 \text { [11] }\end{array}$ & $\begin{array}{l}\text { Mazzeffi, } \\
2016 \text { [18] }\end{array}$ & $\begin{array}{c}\text { Whad } \\
2015 \text { [55] }\end{array}$ & $\begin{array}{l}\text { Suend, } \\
2014[3]\end{array}$ \\
\hline Number of patients & 131 & 48 & 92 & 139 & 86 & $15^{\mathrm{s}}$ & 23 & 24 & 37 \\
\hline PCS & $9,6.9 \%$ & $\ldots$ & $\ldots$ & $10072 \%$ & $\ldots$ & $\ldots$ & $\ldots$ & $\ldots$ & $25,675 \%$ \\
\hline CABG & $\ldots$ & $148,100 \%$ & $12,13 \%$ & $45,45 \%$ & $19,2.1 \%$ & $3,20 \%$ & $7,304 \%$ & $20,83.3 \%$ & ... \\
\hline Valve & $\ldots$ & $\ldots$ & $64,69 \%$ & $19,19 \%$ & $11, \mathrm{n} \cdot 7 \%$ & $4,26.6 \%$ & $5,218 \%$ & $2,8.3 \%$ & $\cdots$ \\
\hline MR (AAD) & $\cdots$ & $\cdots$ & $12,13 \%$ & $\cdots$ & $\ldots$ & $2,13.3 \%$ & $\cdots$ & $\cdots$ & $\cdots$ \\
\hline LVAD & $\cdots$ & $\cdots$ & $2,2 \%$ & $\cdots$ & $\cdots$ & $\ldots$ & $\cdots$ & $\cdots$ & $\cdots$ \\
\hline CABG + value & $\cdots$ & ... & $\ldots$ & $16,16 \%$ & $18,20.9 \%$ & $4,266 \%$ & $\cdots$ & $\cdots$ & $\cdots$ \\
\hline CABG + other & $\ldots$ & $\ldots$ & $\ldots$ & $8,8 \%$ & $1,11 \%$ & $\ldots$ & $\ldots$ & $\ldots$ & $\ldots$ \\
\hline Other & & $\ldots$ & $22 \%$ & $12,12 \%$ & $37,43 \%$ & $2,13.3 \%$ & $11,4.8 \%$ & $2.8 .3 \%$ & $\ldots$ \\
\hline Non-PCS & & $\cdots$ & $\cdots$ & $39,25 \%$ & $\cdots$ & $\cdots$ & $\cdots$ & $\cdots$ & $12,32.5 \%$ \\
\hline AMI & $48,36.6 \%$ & ... & $\cdots$ & $19,14 \%$ & $\ldots$ & $\cdots$ & $\cdots$ & $\cdots$ & $\cdots$ \\
\hline $\mathrm{DOM}$ & $28,21.3 \%$ & $\ldots$ & $\ldots$ & $139 \%$ & $\ldots$ & $\ldots$ & $\ldots$ & $\cdots$ & $\cdots$ \\
\hline Myocardits & $\ldots$ & $\cdots$ & $\cdots$ & $5,4 \%$ & $\cdots$ & $\ldots$ & $\ldots$ & $\ldots$ & $\ldots$ \\
\hline Other & $46,35 \%$ & $\ldots$ & $\ldots$ & $21 \%$ & $\ldots$ & $\ldots$ & $\ldots$ & $\ldots$ & $7,19 \%$ \\
\hline Age, years & 56.4 & 6.4 & 65.4 & 58 & 65 & 643 & 57 & 6.9 & 59 v $70^{\circ}$ \\
\hline Male, $\approx$ & $67.9 \%$ & 8.4 & 59 & 76 & 65 & 733 & 60.9 & 79.2 & $60 \mathrm{vs} 50^{\circ}$ \\
\hline Elective status, $\gamma$ & $\ldots$ & 128 & 64 & 63 & 61.6 & 533 & $\ldots$ & $\ldots$ & ... \\
\hline Eur aSCORE II $\gamma$ & $\ldots$ & $\mathbf{9 . 2}$ & $6.2 \mathrm{vs} 9.4^{4}$ & $\ldots$ & 65 & $19.62^{\circ}$ & $\ldots$ & $\ldots$ & $\ldots$ \\
\hline EOMO at surgery, $*$ & $\ldots$ & 5.4 & 86.9 & 40 & 55.8 & $\ldots$ & $\ldots$ & 3.5 & $\ldots$ \\
\hline$A M C$ & $16,122 \%$ & $19,128 \%$ & $\ldots$ & $\ldots$ & $\ldots$ & $\ldots$ & $\ldots$ & $\ldots$ & $\ldots$ \\
\hline Disul parfusion $\%$ & $108 \%$ & 4.6 & $\ldots$ & 100 & $\cdots$ & $\cdots$ & $\cdots$ & $75 \%$ & 100 \\
\hline LV venting $\%$ & ... & 34 & 14.1 & $\ldots$ & $\ldots$ & $\ldots$ & 0 & $\cdots$ & $\ldots$ \\
\hline INBP, N & $\ldots$ & 25.9 & 27.1 & 83 & 27.1 & $\ldots$ & 20 & 85 & 8.1 \\
\hline EQMO duration, days & $4^{t}$ & 64 & $6^{4}$ & 4.8 & $s^{t}$ & $5.4^{\mathrm{s}}$ & $3^{t}$ & 47 & $5.8 \mathrm{vs} 6^{\circ}$ \\
\hline EOMO weaning, * & $\ldots$ & 486 & 48 & 63 & 49 & $\ldots$ & 60.8 & 6.7 & $\ldots$ \\
\hline Gl complicators, $\%$ & 6.1 & 20.8 & $\ldots$ & 25 & 14.3 & $\ldots$ & $\ldots$ & 208 & $\ldots$ \\
\hline $\mathrm{CVE}_{2} \approx$ & 14.5 & 22.2 & 3.2 & 25 & 11.9 & 187 & $\ldots$ & 375 & 16.2 \\
\hline CRKT, $\approx$ & 14.5 & 5.3 & $\ldots$ & 66 & 29.8 & 187 & 47.8 & $\mathbf{2 9 . 2}$ & ... \\
\hline Reop for blending $\%$ & 11.1 & 4.9 & 19.5 & 36 & 46.4 & 333 & $\ldots$ & $\ldots$ & 621 \\
\hline VACflimb ishemia, $\approx$ & 29.5 & 2.1 & 9.7 & 12 & 10.7 & 333 & $\ldots$ & 83 & 18.9 \\
\hline Early oukome & Inhospital V & Inhospital & $\mathrm{NA}$ & $30 \mathrm{~d}$ & Inhospital & Inhospital & $30 \mathrm{~d}$ inhorpital & Inhospital & $30 \mathrm{~d}$ \\
\hline Variables & $\begin{array}{l}\text { Loforte, } \\
2014 \text { [6] }\end{array}$ & $\begin{array}{c}\text { Mikus } \\
2013 \text { [4] }\end{array}$ & $\begin{array}{l}\text { Unos } \\
2012\end{array}$ & $\begin{array}{l}62162, \\
2 \text { [16] }\end{array}$ & $\begin{array}{l}\text { Polversnik, } \\
2012 \text { [13] }\end{array}$ & $\begin{array}{c}K a n j i \\
2010[7]\end{array}$ & $\begin{array}{l}\text { Rastan, } \\
\mathbf{2} 10 \quad[4]\end{array}$ & $\begin{array}{c}\text { Russa, } \\
2010[46]\end{array}$ & $\begin{array}{c}K a \\
2002[9]\end{array}$ \\
\hline Number of patients & 228 & 4 & & 47 & 49 & $\boldsymbol{5}$ & 517 & 15 & 76 \\
\hline PCS & $255,6.9 \%$ & ... & & $\ldots$ & $49,200 \%$ & $37,74 \%$ & $\ldots$ & $3, \mathbf{z} \approx$ & $\ldots$ \\
\hline CABG & $68,4.8 \%$ & $5,35.7 \%$ & 19,4 & $40.4 \%$ & $\ldots$ & ... & $193374 \%$ & $1,66 \%$ & $37,486 \%$ \\
\hline Valve & $50,322 \%$ & $6,42.8 \%$ & & $17 \%$ & $\ldots$ & $\ldots$ & $7414.3 \%$ & $1,66 \%$ & $4,184 \%$ \\
\hline MR (AAD) & $\ldots$ & $\ldots$ & & $\ldots$ & $\ldots$ & $\ldots$ & $\mathbf{2}, 3.5 \%$ & $\ldots$ & $2,26 \%$ \\
\hline LVAD & $\ldots$ & $\ldots$ & & $\ldots$ & $\cdots$ & $\ldots$ & $\ldots$ & $\ldots$ & $2.26 \%$ \\
\hline CABG + valve & $\cdots$ & ... & 2,4 & $4.2 \%$ & $\cdots$ & $\ldots$ & $87,16.8 \%$ & $\cdots$ & $6.7 .8 \%$ \\
\hline CABG + other & $\ldots$ & $\ldots$ & & $6.3 \%$ & $\ldots$ & $\ldots$ & $\ldots$ & $\ldots$ & $\ldots$ \\
\hline Other & $37,23.8 \%$ & $3,21.4 \%$ & 15,3 & $31.9 \%$ & $\ldots$ & $\ldots$ & $143276 \%$ & $1,66 \%$ & $5,197 \%$ \\
\hline Non-PCS & $73,3.1 \%$ & $\ldots$ & & $\ldots$ & $\ldots$ & $13,26 \%$ & $\ldots$ & $12,80 \%$ & $\ldots$ \\
\hline AMI & $27, \mathrm{n} .8 \%$ & $\ldots$ & & $\ldots$ & $\cdots$ & $\ldots$ & $\ldots$ & $2,133 \%$ & $\ldots$ \\
\hline $\mathrm{DOM}$ & $40,7.5 \%$ & $\ldots$ & & $\cdots$ & $\cdots$ & $\ldots$ & $\ldots$ & $4,266 \%$ & $\ldots$ \\
\hline Myocandits & $6,26 \%$ & $\ldots$ & & ... & $\ldots$ & $\ldots$ & $\ldots$ & $2,133 \%$ & $\ldots$ \\
\hline Other & $\ldots$ & $\ldots$ & & $\ldots$ & $\ldots$ & $\ldots$ & $\ldots$ & $4,266 \%$ & $\ldots$ \\
\hline Age, years & 58.3 & $\$ 3.1$ & & 44 & 65 & 46 vs $52^{\circ}$ & 63.5 & 44.7 & 56.8 \\
\hline
\end{tabular}


Table 1. Continued

\begin{tabular}{|c|c|c|c|c|c|c|c|c|}
\hline Variables & $\begin{array}{l}\text { Loforte, } \\
2014 \text { [6] }\end{array}$ & $\begin{array}{c}\text { Mikus, } \\
2013 \text { [14] }\end{array}$ & $\begin{array}{l}\text { Unosawa, } \\
2012 \text { [16] }\end{array}$ & $\begin{array}{l}\text { Pokersnik, } \\
2012 \text { [13] }\end{array}$ & $\begin{array}{c}\text { Kanji, } \\
2010[7]\end{array}$ & $\begin{array}{l}\text { Rastan, } \\
2010[4]\end{array}$ & $\begin{array}{c}\text { Russo, } \\
2010 \text { [46] }\end{array}$ & $\begin{array}{c}\text { Ko, } \\
2002[9]\end{array}$ \\
\hline Male, $\%$ & 67.9 & 64.3 & 35 & 33 & 72 & 71.5 & 66.6 & 63 \\
\hline Elective status, $\%$ & $\ldots$ & 64.2 & 53.1 & 100 & $\cdots$ & 36.7 & $\ldots$ & $\ldots$ \\
\hline EuroSCORE II, \% & $25.7^{e}$ & $\ldots$ & $9.72^{e}$ & $\ldots$ & $\ldots$ & $21.6^{e}$ & $\ldots$ & $\ldots$ \\
\hline ECMO at surgery, $\%$ & 88.5 & 85.7 & 70.2 & $\ldots$ & ... & 41.9 & $\ldots$ & 51.3 \\
\hline $\mathrm{AAC}$ & $\ldots$ & $\ldots$ & $\ldots$ & Yes & $\ldots$ & $62,11.9 \%$ & $\ldots$ & $\ldots$ \\
\hline Distal perfusion, $\%$ & 65.8 & 100 & $100^{\mathrm{g}}$ & Frequently & $\ldots$ & 23.4 & 66.6 & 26.3 \\
\hline LV venting, \% & $\ldots$ & $100^{h}$ & 0 & $\ldots$ & $\ldots$ & $\ldots$ & 6.6 & 2.6 \\
\hline IABP, $\%$ & 100 & 92.8 & 83 & 60.6 vs $56.3^{d}$ & 73 vs $71^{c}$ & 74.1 & 80 & 69 \\
\hline ECMO duration, days & 10.9 & 5 & 2.6 & 3.8 vs $4.3^{\mathrm{d}}$ & 3 vs $2.5^{c}$ & 3.28 & 11.5 & 4.1 \\
\hline ECMO weaning, \% & 46.9 & 50 & 55.3 & $63.6,45.5,55.6^{i}$ & $\ldots$ & 63.3 & 80 & 60.5 \\
\hline GI complications, $\%$ & $\ldots$ & $\ldots$ & $\ldots$ & $\ldots$ & $\ldots$ & 18.8 & $\ldots$ & $\ldots$ \\
\hline CVE, \% & 15.7 & 14.2 & $\ldots$ & 6.1 & 10 & 9 & $\mathbf{0}$ & 3.9 \\
\hline CRRT, \% & 49.5 & 57.1 & 31.9 & 32.6 & $\ldots$ & 65 & $\ldots$ & 36.8 \\
\hline Reop for bleeding, \% & 48.4 vs $62.7^{c}$ & 64.2 & 42.5 & 78.8 vs $56.3^{d}$ & 44 & 58 & 26.6 & 46 \\
\hline VAC/limb ischemia, $\%$ & 5.7 & 0 & 10.6 & $\ldots$ & 14 & 19.9 & $\ldots$ & 17.1 \\
\hline Early outcome & Inhospital & Inhospital & Inhospital & Inhospital & $30 \mathrm{~d}$ & Inhospital & Inhospital & Inhospital \\
\hline
\end{tabular}

"Including thoracic organs transplantation. $\quad{ }^{b}$ One patient had right ventricular assist device. 'Peripheral extracorporeal membrane oxygenation (ECMO) versus central ECMO. ¿Survivors versus nonsurvivors. "Logistic European System for Cardiac Operative Risk Evaluation (EuroSCORE). $\quad{ }^{\prime}$ Median. ${ }^{8}$ Distal limb perfusion perfomed in $100 \%$ of cases after year $1999 .{ }^{\mathrm{h}}$ All in cECMO. ${ }^{1}$ According to centrifugal pump and oxygenator combination. i Weaning or bridging.

Continuous variables are mean and categoric variables are percentage.

$\mathrm{AAC}=$ axillary arterial cannulation; $\quad \mathrm{AAD}=$ acute aortic dissection; $\quad \mathrm{AAR}=$ ascending aorta replacement; $\quad \mathrm{AMI}=$ acute myocardial infarc tion; $\quad C A B G=$ comonary artery bypass grt surgery; $\quad$ CRRT $=$ continuous renal replacement therapy; $\quad C V E=$ cerebrovascular event $\quad \mathrm{d}=$ days; $\quad \mathrm{DCM}=$ decompensated cardiomyopathy; $\quad \mathrm{ECMO}=$ extracorporeal membrane oxygenation; $\quad \mathrm{GI}=$ gastrointestinal; $\quad \mathrm{IABP}=$ intraaortic balloon pump; $\quad \mathrm{LV}=$ left ventricular; $\quad$ LVAD = left ventricle assist device; $\quad \mathrm{PCS}=$ postcardiotomy shock; $\quad \mathrm{Reop}=$ reoperation; VAC $=$ vascular access complications; $\quad$ vs $=$ versus.

Risk of bias for each study across each of the seven risk of bias domains is presented in Appendix Table 1. Overall, the studies reported either moderate or serious risk of bias. Given the overall high risk of bias along with the limited number of studies, all articles were retained for the purposes of this review. Most commonly, biases arose from 1) selection of participants for the study, and 2) subjective distribution of the participants within the study arms by either designated heart teams or according to surgeon preference and underlying causes. 

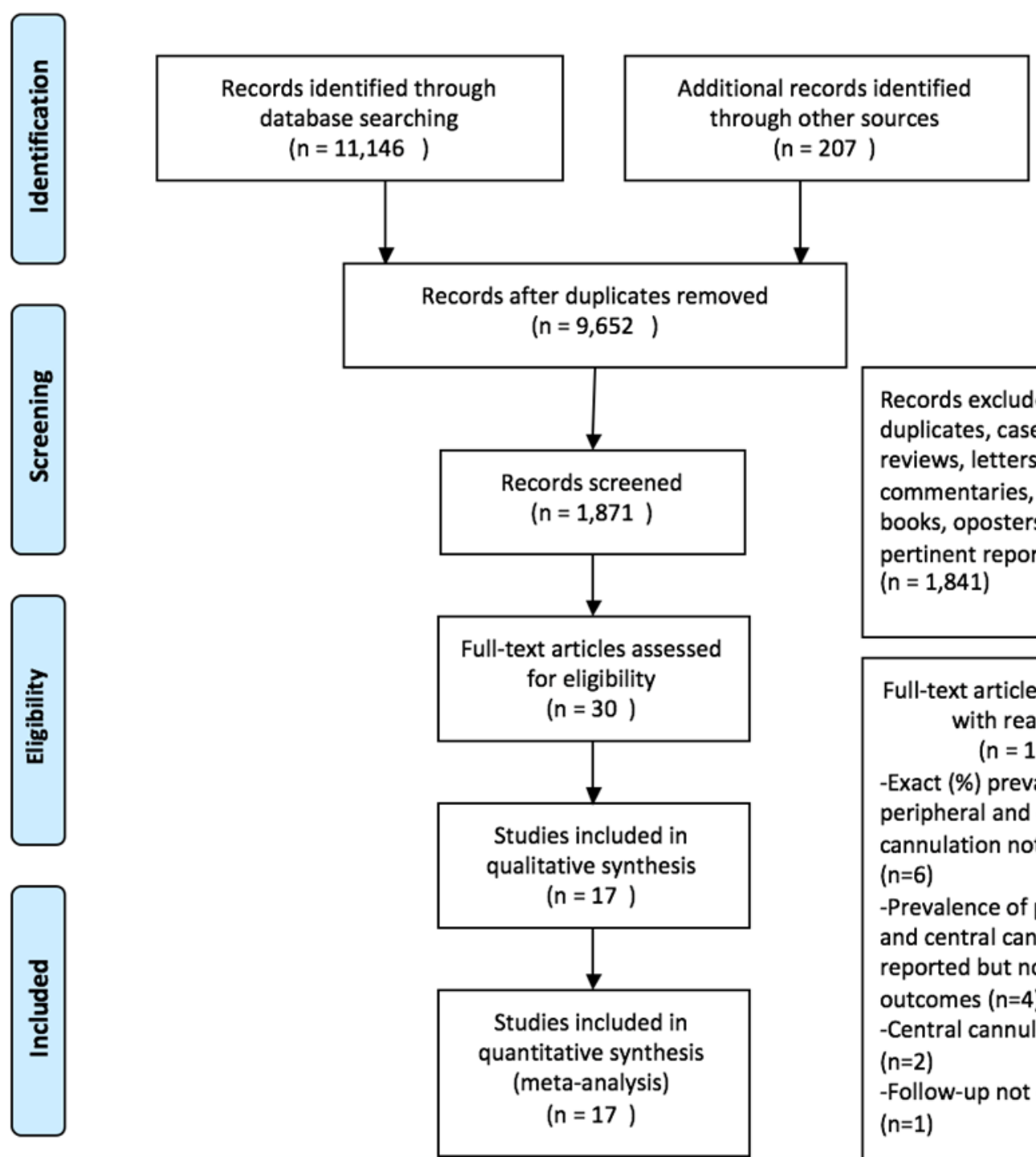

Records excluded:

duplicates, case reports, reviews, letters,

commentaries, editorials, books, oposters, other not pertinent reports ( $n=1,841$ )

Full-text articles excluded, with reasons ( $n=13$ )

-Exact (\%) prevalence of peripheral and central cannulation not reported $(n=6)$

-Prevalence of peripheral and central cannulation reported but no clinical outcomes $(n=4)$

-Central cannulation only $(n=2)$

-Follow-up not of interest $(n=1)$

sFigure 1. Flow diagram of the review process along with reasons for exclusion according to the Preferred Reporting Items for Systematic Reviews and Meta-analyses (PRISMA) statement. 
Appendix Table 1. ROBINS-I tool bias assessment.

\begin{tabular}{|c|c|c|c|c|c|c|c|c|c|}
\hline Study & $\begin{array}{l}\text { Bias due to } \\
\text { confounding }\end{array}$ & $\begin{array}{l}\text { Bias in selection } \\
\text { of participants } \\
\text { into the study }\end{array}$ & $\begin{array}{l}\text { Bias in } \\
\text { measurement of } \\
\text { interventions }\end{array}$ & $\begin{array}{l}\text { Bias due to } \\
\text { departures from } \\
\text { intended } \\
\text { interventions }\end{array}$ & $\begin{array}{l}\text { Bias due to } \\
\text { missing } \\
\text { data* }\end{array}$ & $\begin{array}{l}\text { Bias in } \\
\text { measurement of } \\
\text { outcomes* }\end{array}$ & $\begin{array}{l}\text { Bias in } \\
\text { selection of } \\
\text { reported } \\
\text { result* }\end{array}$ & $\begin{array}{l}\text { Overall } \\
\text { bias }\end{array}$ & $\begin{array}{l}\text { Cohen's } \\
\text { Kappa }\end{array}$ \\
\hline $\begin{array}{l}\text { Ranney DN, } \\
2017^{10}\end{array}$ & Moderate & Serious & Serious & Moderate & Critical & Low & Low & Serious & 0.86 \\
\hline $\begin{array}{l}\text { Biancari F, } \\
2017^{12}\end{array}$ & Moderate & Serious & Moderate & Moderate & Moderate & Low & Low & Moderate & 0.71 \\
\hline $\begin{array}{l}\text { Guihaire J, } \\
2017^{17}\end{array}$ & Moderate & Serious & Serious & Moderate & Critical & Low & Moderate & Serious & 0.71 \\
\hline $\begin{array}{l}\text { Slottosch I, } \\
2017^{15}\end{array}$ & Moderate & Serious & Serious & Moderate & Critical & Low & Low & Serious & 0.71 \\
\hline $\begin{array}{l}\text { Raffa GM, } \\
2017^{4}\end{array}$ & Moderate & Serious & Serious & Moderate & Critical & Low & Low & Serious & 0.86 \\
\hline $\begin{array}{l}\text { Khorsandi M, } \\
2016^{11}\end{array}$ & Moderate & Serious & Moderate & Moderate & Moderate & Low & Low & Moderate & 0.57 \\
\hline $\begin{array}{l}\text { Zhao Y, } \\
2015^{45}\end{array}$ & Moderate & Serious & Moderate & Moderate & Moderate & Low & Serious & Moderate & 0.71 \\
\hline $\begin{array}{l}\text { Mazzeffi MA, } \\
2016^{18}\end{array}$ & Moderate & Serious & Serious & Moderate & Critical & Low & Low & Serious & 0.86 \\
\hline $\begin{array}{l}\text { Saeed D, } \\
2014^{8}\end{array}$ & Moderate & Serious & Moderate & Moderate & Moderate & Low & Serious & Moderate & 0.57 \\
\hline $\begin{array}{l}\text { Loforte A, } \\
2014^{6}\end{array}$ & Moderate & Serious & Moderate & Moderate & Moderate & Low & Moderate & Moderate & 0.71 \\
\hline $\begin{array}{l}\text { Mikus E, } \\
2013^{14}\end{array}$ & Moderate & Serious & Moderate & Moderate & Moderate & Low & Serious & Moderate & 0.57 \\
\hline $\begin{array}{l}\text { Unosawa S, } \\
2012^{16}\end{array}$ & Moderate & Serious & Serious & Moderate & Critical & Low & Moderate & Serious & 0.86 \\
\hline $\begin{array}{l}\text { Pokersnik JA, } \\
2012^{13}\end{array}$ & Moderate & Serious & Serious & Moderate & Critical & Low & Moderate & Serious & 0.71 \\
\hline $\begin{array}{l}\text { Kanji HD, } \\
2010^{7}\end{array}$ & Moderate & Serious & Moderate & Moderate & Moderate & Low & Moderate & Moderate & 0.71 \\
\hline $\begin{array}{l}\text { Rastan AJ, } \\
2010^{4}\end{array}$ & Moderate & Serious & Serious & Moderate & Critical & Low & Low & Serious & 0.57 \\
\hline $\begin{array}{l}\text { Russo CF, } \\
2010^{46}\end{array}$ & Modearte & Serious & Serious & Moderate & Moderate & Low & Serious & Moderate & 0.86 \\
\hline $\begin{array}{l}\text { Ko WJ, } \\
2002^{9}\end{array}$ & Moderate & Serious & Moderate & Moderate & Moderate & Low & Moderate & Moderate & 0.57 \\
\hline
\end{tabular}




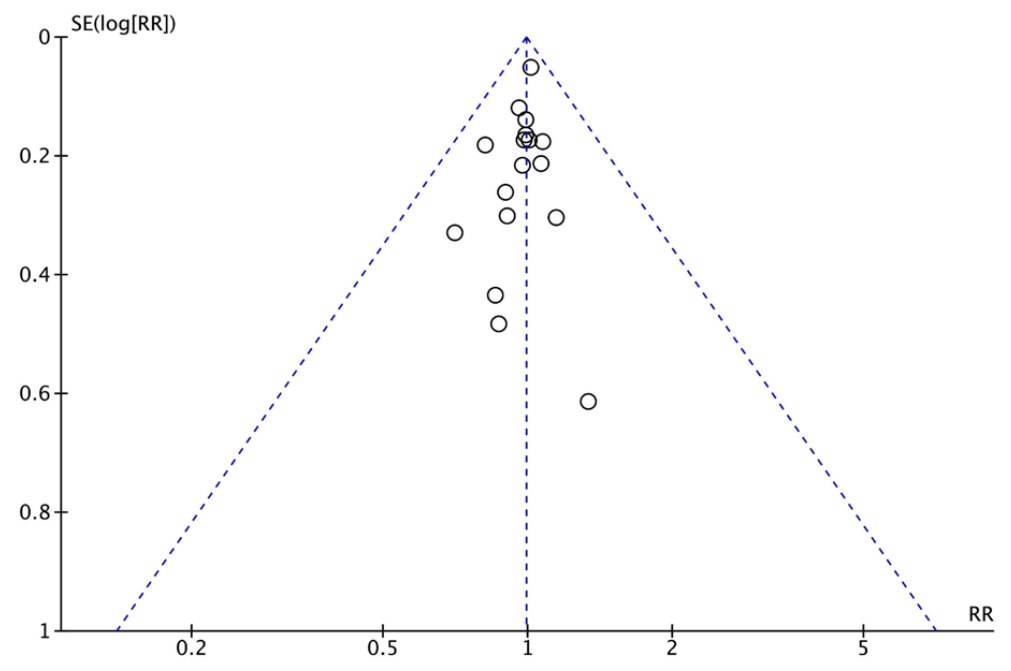

sFigure 2. Publication bias analysis. SE, Standard error; RR, risk ratio.

\begin{tabular}{|c|c|c|c|c|c|c|c|c|}
\hline Study or Subgroup & \multicolumn{2}{|c|}{ Peripheral ECMO } & \multicolumn{2}{|c|}{ Central ECMO } & Weight & \multirow[t]{2}{*}{$\begin{array}{c}\text { Risk Ratio } \\
\text { IV, Random, } 95 \% \mathrm{CI}\end{array}$} & \multicolumn{2}{|c|}{$\begin{array}{c}\text { Risk Ratio } \\
\text { IV, Random, } 95 \% \mathrm{CI}\end{array}$} \\
\hline \multicolumn{8}{|c|}{$\begin{array}{l}\text { 1.3.1 post coronary surgery } \\
\end{array}$} & \\
\hline Biancari $\mathrm{F}$ et al. 2017 & 64 & 89 & 39 & 59 & $10.4 \%$ & $1.09[0.87,1.36]$ & & - \\
\hline Ko WJ et al. 2002 & 45 & 61 & 11 & 15 & $4.5 \%$ & $1.01[0.72,1.41]$ & & \\
\hline Slottosch I et al. 2017 & 75 & 110 & 20 & 29 & $6.9 \%$ & $0.99[0.75,1.30]$ & & - \\
\hline Unosawa S et al. 2012 & 21 & 32 & 12 & 15 & $4.1 \%$ & $0.82[0.57,1.17]$ & & - \\
\hline $\begin{array}{l}\text { Zhao Y et al. } 2015 \\
\text { Subtotal }(95 \% C I)\end{array}$ & 15 & 315 & 1 & 11 & $\begin{array}{r}0.7 \% \\
26.8 \%\end{array}$ & $0.86[0.37,2.02]$ & & - \\
\hline Subtotal $(95 \% \mathrm{CI})$ & & 315 & & 119 & $26.8 \%$ & $1.00[0.87,1.15]$ & & \\
\hline \multirow{2}{*}{\multicolumn{9}{|c|}{$\begin{array}{l}\text { Total events } \\
\text { Heterogeneity: } \text { Tau }^{2}=0.00 ; \mathrm{Chi}^{2}=1.85, \mathrm{df}=4(\mathrm{P}=0.76) ; \mathrm{I}^{2}=0 \% \\
\text { Test for overall effect: } Z=0.05(P=0.96)\end{array}$}} \\
\hline & & & & & & & & \\
\hline \multicolumn{9}{|c|}{ 1.3.2 post valvular surgery } \\
\hline Guihaire J et al. 2017 & 49 & 78 & 9 & 14 & $2.9 \%$ & $0.98[0.64,1.50]$ & & \\
\hline Mikus E et al. 2013 & 3 & 6 & 3 & 8 & $0.4 \%$ & $1.33[0.40,4.43]$ & & \\
\hline Subtotal $(95 \% \mathrm{CI})$ & & 84 & & 22 & $3.3 \%$ & $1.01[0.68,1.51]$ & & \\
\hline \multirow{2}{*}{\multicolumn{9}{|c|}{$\begin{array}{l}\text { Total events } \\
\text { Heterogeneity: } \operatorname{Tau}^{2}=0.00 ; \mathrm{Ch}^{2}=0.23, \mathrm{df}=1(\mathrm{P}=0.63) ; \mathrm{I}^{2}=0 \% \\
\text { Test for overall effect: } Z=0.06(P=0.95)\end{array}$}} \\
\hline & & & & & & & & \\
\hline \multicolumn{9}{|c|}{ 1.3.3 post combined heart surgery and other ${ }^{*}$} \\
\hline Kanji HD et al. 2010 & 10 & 22 & 14 & 28 & $1.5 \%$ & $0.91[0.50,1.64]$ & & \\
\hline Khorsandi M et al. 2016 & 4 & 5 & 7 & 10 & $1.5 \%$ & $1.14[0.63,2.08]$ & & \\
\hline Loforte A et al. 2014 & 48 & 126 & 36 & 102 & $4.4 \%$ & $1.08[0.76,1.52]$ & & \\
\hline Mazzeffi MA et al. 2016 & 5 & 9 & 11 & 14 & $1.3 \%$ & $0.71[0.37,1.35]$ & & - \\
\hline Pokersnik JA et al. 2012 & 22 & 32 & 11 & 17 & $3.0 \%$ & $1.06[0.70,1.62]$ & & \\
\hline Raffa CM et al. 2017 & 35 & 56 & 19 & 30 & $4.6 \%$ & $0.99[0.70,1.39]$ & & \\
\hline Rastan AJ et al. 2010 & 154 & 203 & 235 & 314 & $51.8 \%$ & $1.01[0.92,1.12]$ & & \\
\hline Russo CF et al. 2010 & 0 & 1 & 0 & 2 & & Not estimable & & \\
\hline Saeed D et al. 2014 & 15 & 25 & 8 & & $2.0 \%$ & $0.90[0.54,1.50]$ & & \\
\hline Subtotal $(95 \% \mathrm{Cl})$ & & 479 & & 529 & $70.0 \%$ & $1.01[0.92,1.10]$ & & \\
\hline \multirow{3}{*}{\multicolumn{9}{|c|}{$\begin{array}{l}\text { Heterogeneity: } \text { Tau }^{2}=0.00 ; \text { Chi }^{2}=1.87, \mathrm{df}=7(\mathrm{P}=0.97) ; 1^{2}=0 \% \\
\text { Test for overall effect: } Z=0.18(P=0.86)\end{array}$}} \\
\hline & & & & & & & & \\
\hline & & & & & & & & \\
\hline Total $(95 \% \mathrm{Cl})$ & & 878 & & 670 & $100.0 \%$ & $1.01[0.93,1.08]$ & & 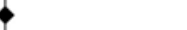 \\
\hline \multicolumn{7}{|c|}{$\begin{array}{l}\text { Total events } \\
\text { Heterogeneity: } \mathrm{Tau}^{2}=0.00 ; \mathrm{Ch}^{2}=3.9\end{array}$} & & \\
\hline $\begin{array}{l}\text { Heterogeneity: } \operatorname{Tau}^{2}=0 \text {. } \\
\text { Test for overall effect: } Z \text {, }\end{array}$ & $\begin{array}{l}0 ; \mathrm{Ch}^{2}=3 \\
0.14(P=\end{array}$ & $\begin{array}{l}8, \mathrm{df}= \\
\text { 89) }\end{array}$ & $14(P=1$. & & & & $0.2 \quad 0.5{ }_{\text {Favours Peripheral ECMO }}^{1}$ & i $\frac{1}{2}$ \\
\hline
\end{tabular}

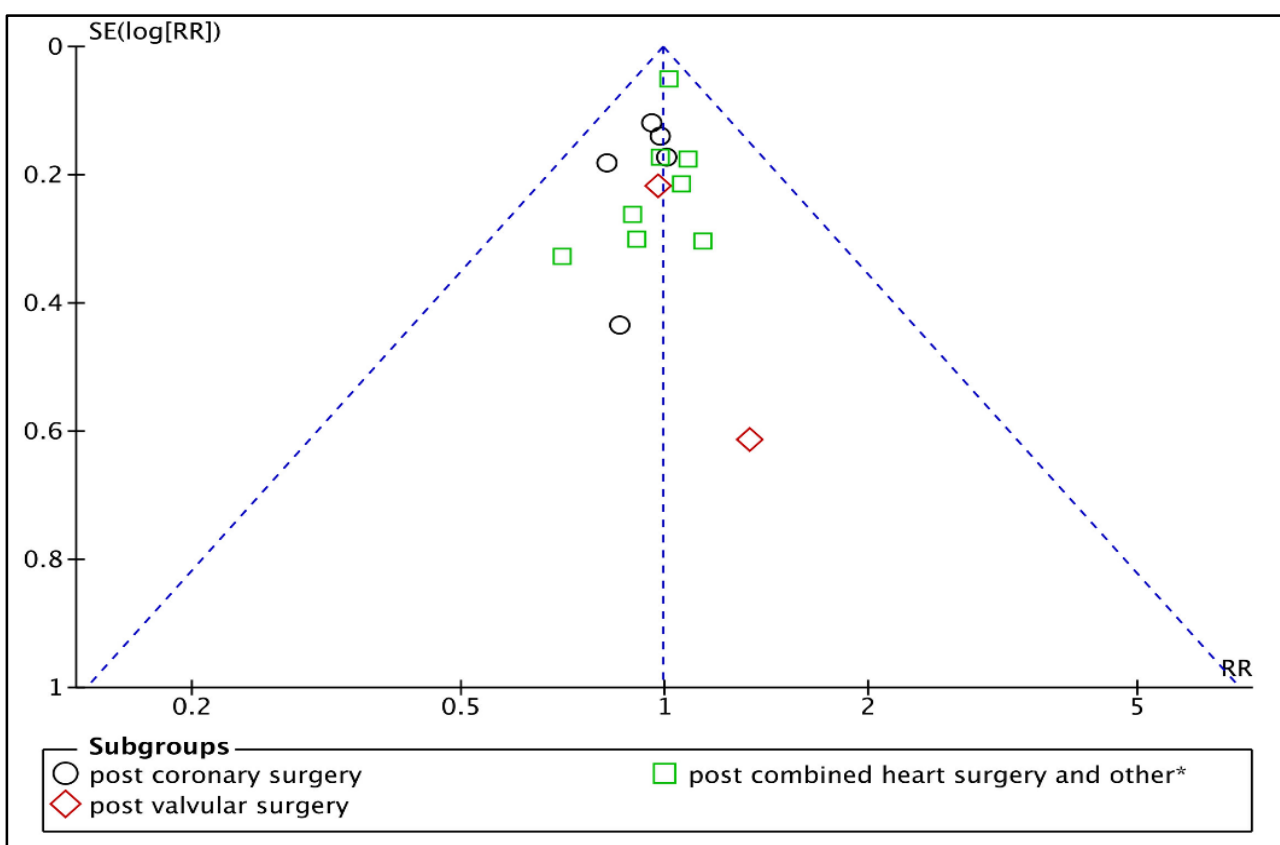


sFigure 3. Prespecified sensitivity analysis stratified by surgery type (A) and publication bias analysis in corresponding subgroups (B). SE, Standard error; RR, risk ratio.

*- combined coronary and valvular surgery, along with surgery on thoracic aorta and other cardiac surgery. The stratification was performed according to majority of patients undergoing given surgical procedure across single studies.

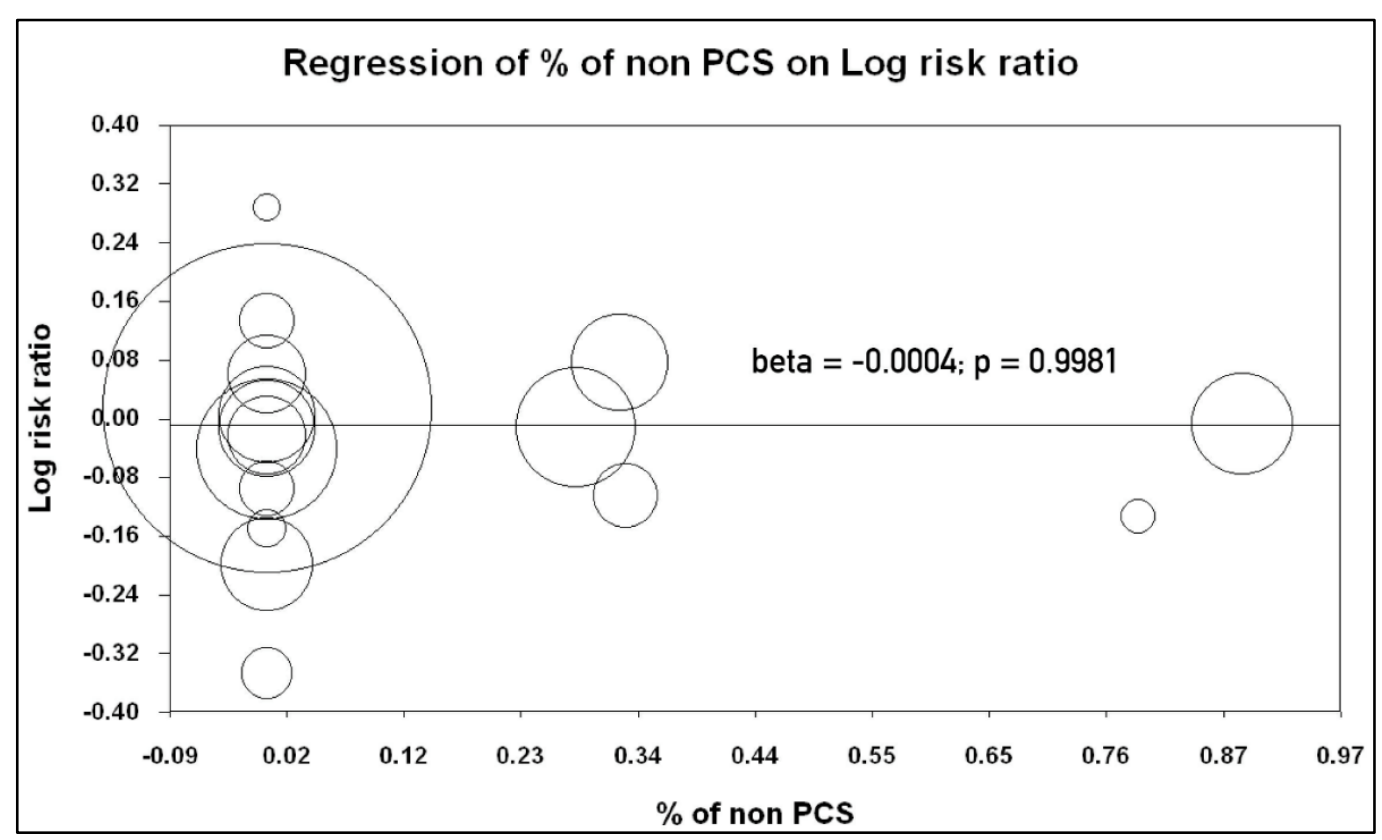

sFigure 4. Weighted random-effects Meta regression analysis on the percentage of nonpostcardiotomy patients and the relationship with log RR of primary endpoint in-hospital mortality. The size of the circle corresponds to the inverse variance of the log-RR, and thus is related to the statistical weight of the individual study. 


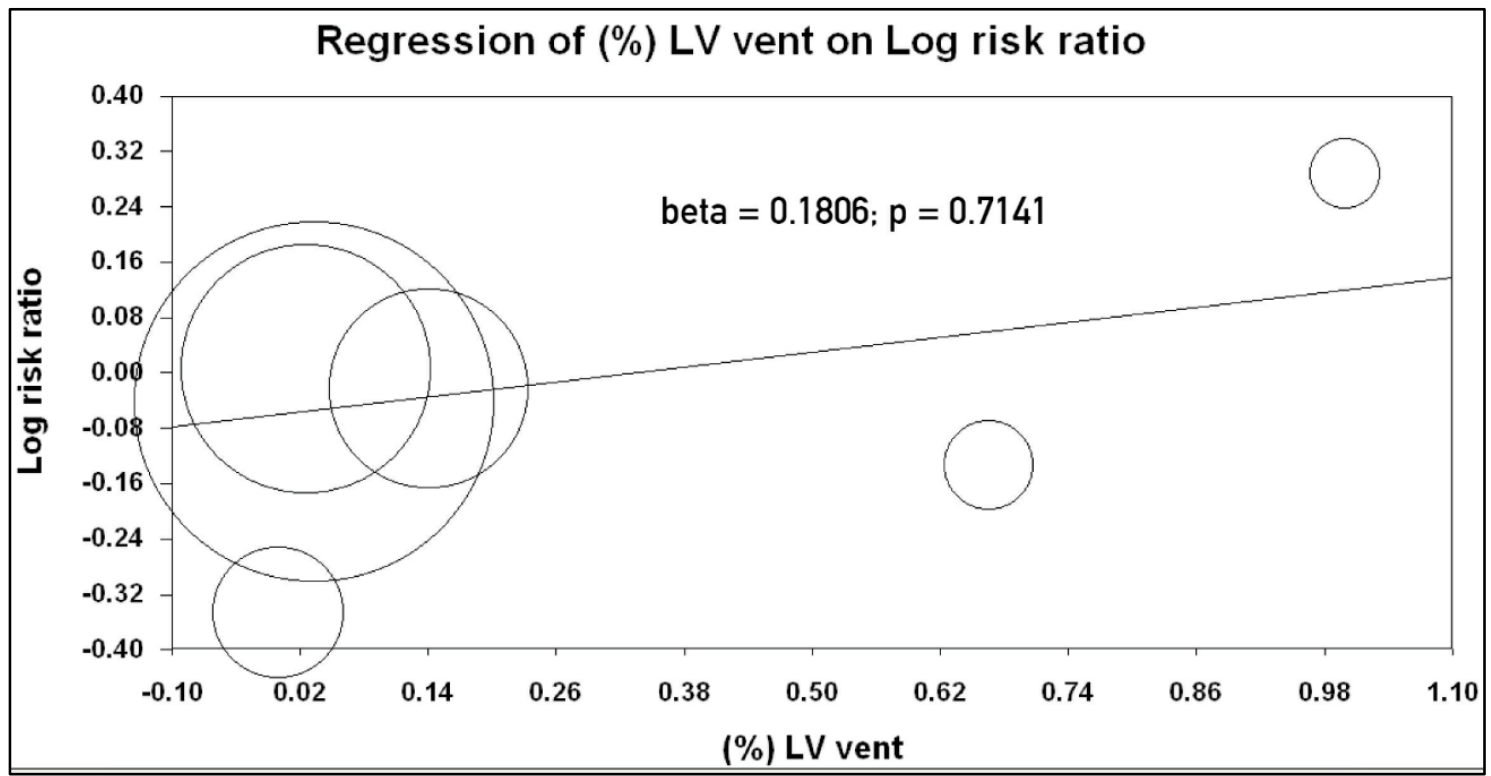

sFigure 5. Weighted random-effects Meta regression analysis on the percentage of left ventricle (LV) venting in the entire studied group and the relationship with $\log \mathrm{RR}$ of primary endpoint in-hospital mortality. The size of the circle corresponds to the inverse variance of the log-RR, and thus is related to the statistical weight of the individual study.

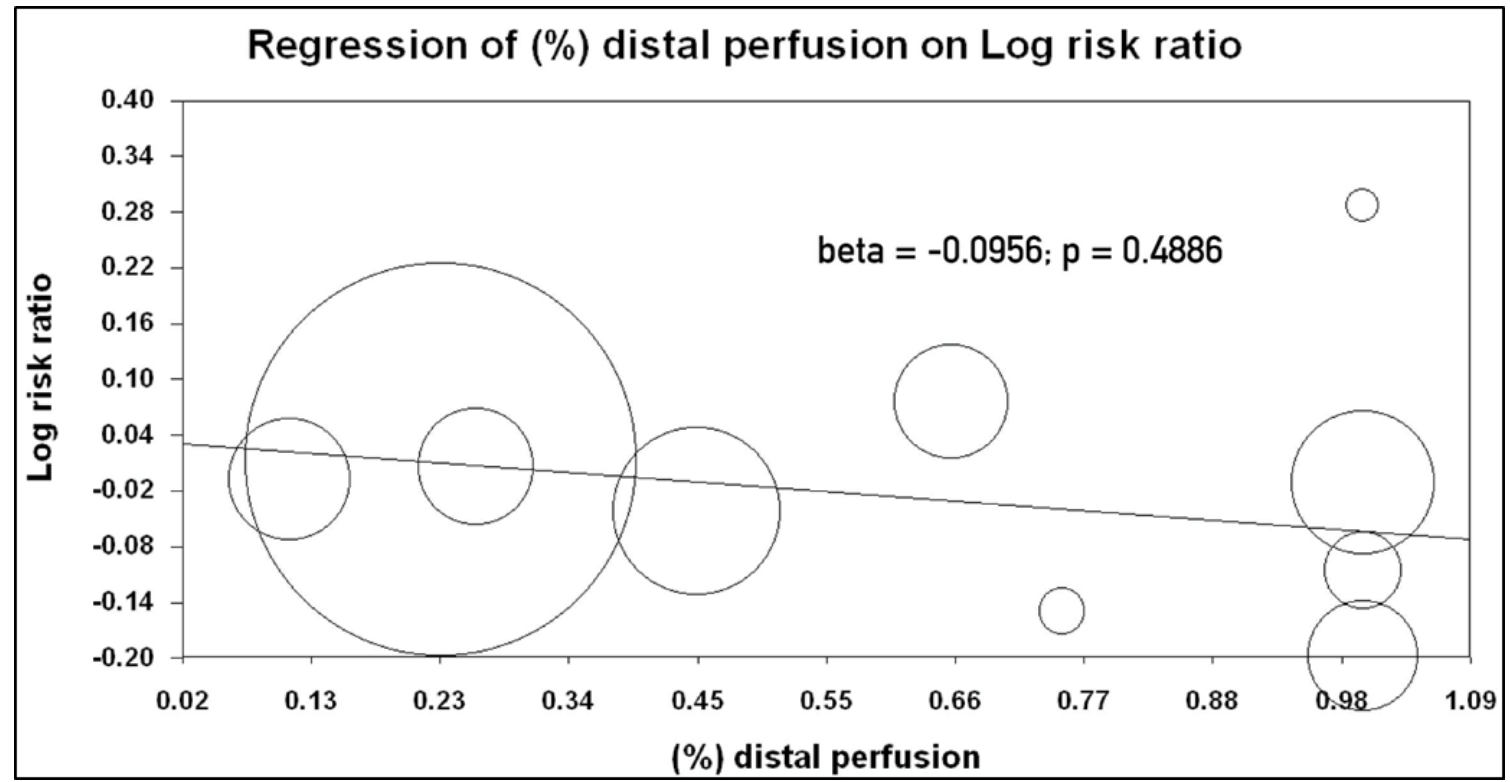

sFigure 6. Weighted random-effects Meta regression analysis on the percentage of distal perfusion (DP) in the entire studied group and the relationship with $\log \mathrm{RR}$ of primary endpoint in-hospital mortality. The size of the circle corresponds to the inverse variance of the log-RR, and thus is related to the statistical weight of the individual study. 


\section{Secondary endpoints.}

A total of six studies enrolling 323 patients contributed to the analysis of cerebrovascular events. No statistically significant differences were found in regard to risk of CVE: $0.88(0.46-1.68) ; P=0.69$; $I^{2}=0 \%$ with corresponding event rates of $10.7 \%(23 / 214)$ vs $13.8 \%(15 / 109)$ for peripheral and central VA-ECMO, respectively (Figure 2A).

Seven studies (699 patients) provided data for the analysis of bleeding. Bleeding occurred in over $40 \%$ of VA-ECMO patients; peripheral, as compared to central, cannulation was associated with a significant $35 \%$ reduction of the bleeding risk $\left(0.65[0.46-0.93] ; P=0.02 ; I^{2}=59 \%\right)$. Respective bleeding rates were $32.9 \%$ (141/429) in peripheral and $51.9(140 / 270)$ using a central cannulation approach (Figure 2B).

Peripheral cannulation for VA-ECMO reduced the need for CVVH by nearly $25 \%(0.76$ [0.60-0.97]; $P=0.03 ; I^{2}=0 \%$ ) when compared to central cannulation. Accordingly, CVVH was used in $30.2 \%$ of cases in the peripheral cannulation group (70/232) as compared to those patients with central cannulation where the incidence of CVVH reached 45.5\% (71/156) (Figure 2C). With six studies and 537 patients included, there were no differences in the risk of limb complications between peripheral and central VA-ECMO: $1.68(0.63-4.46) ; P=0.30 ; I^{2}=53 \%$ (Figure 2D). Similarly, no differences between cannulation approaches were observed in the analysis of sepsis: $0.71(0.27-1.88) ; P=0.50$; $I^{2}=11 \%($ Figure $2 \mathrm{E})$.

Lastly, peripheral cannulation as compared to central cannulation was associated with a significantly reduced number of transfusions administered (Figure 3). Number of transfused packed red blood cells was significantly lower (by over 7 units) with peripheral cannulation: weighted MD (mean difference) (95\%CIs): $-7.17[-9.94,-4.40] ; P<0.00001 ; I^{2}=36 \%$; as compared to central cannulation. Similarly, the number of units of fresh frozen plasma administered was significantly lower (by nearly 3 units) when peripheral cannulation was employed as compared to central cannulation: -2.73 [-4.16, -1.29]; $P=0.0002 ; I^{2}=33 \%$. Number of transfused units of platelets was similarly reduced with peripheral cannulation: $-5.09[-6.77,-3.40] ; P<0.00001 ; I^{2}=0 \%$ by over 5 units on average. 


\begin{tabular}{|c|c|c|c|c|c|c|c|c|c|c|}
\hline Study or Subgroup & \multicolumn{2}{|c|}{ Peripheral ECMO } & \multicolumn{2}{|c|}{ Central ECMO } & Weight & $\begin{array}{c}\text { Risk Ratio } \\
\text { IV, Random, } 95 \% \mathrm{CI} \\
\end{array}$ & \multicolumn{4}{|c|}{$\begin{array}{c}\text { Risk Ratio } \\
\text { IV, Random, } 95 \% \mathrm{Cl} \\
\end{array}$} \\
\hline Kanji HD et al. 2010 & 3 & 22 & 2 & 28 & $30.7 \%$ & $1.91[0.35,10.45]$ & & & $=$ & \\
\hline Khorsandi M et al. 2016 & 0 & 5 & 3 & 10 & $11.4 \%$ & $0.26[0.02,4.27]$ & & & & \\
\hline Ko WJ et al. 2002 & 3 & 61 & 0 & 15 & $10.4 \%$ & $1.81[0.10,33.22]$ & & & - & \\
\hline Mikus E et al. 2013 & 0 & 6 & 2 & 8 & $10.7 \%$ & $0.26[0.01,4.54]$ & & $\because$ & & \\
\hline Saeed D et al. 2014 & 4 & 25 & 2 & 12 & $36.8 \%$ & $0.96[0.20,4.53]$ & & & & \\
\hline Total $(95 \% \mathrm{Cl})$ & & 119 & & 73 & $100.0 \%$ & $0.95[0.37,2.43]$ & & & & \\
\hline Total events & 10 & & 9 & & & & & & & \\
\hline \multicolumn{7}{|c|}{$\begin{array}{l}\text { Heterogeneity: } \text { Tau }^{2}=0.00 ; \mathrm{Chi}^{2}=2.45, \mathrm{df}=4(\mathrm{P}=0.65) ; \mathrm{I}^{2}=0 \% \\
\text { Test for overall effect: } \mathrm{Z}=0.11(\mathrm{P}=0.91)\end{array}$} & \multicolumn{2}{|c|}{$0.01 \quad 0.1$} & $\begin{array}{c}10 \\
\text { Favours Central ECMO }\end{array}$ & $\overrightarrow{100}$ \\
\hline A & \multicolumn{2}{|c|}{ Peripheral ECMO } & \multicolumn{3}{|c|}{ Central ECMO } & Risk Ratio & \multirow{2}{*}{\multicolumn{4}{|c|}{$\begin{array}{c}\text { Risk Ratio } \\
\text { IV, Random, } 95 \% \mathrm{Cl} \\
\end{array}$}} \\
\hline Study or Subgroup & Events & Total E & Events & Total & Weight & IV, Random, 95\% CI & & & & \\
\hline Biancari F et al. 2017 & 37 & 89 & 29 & 59 & $22.3 \%$ & $0.85[0.59,1.21]$ & & & T & \\
\hline Kanji HD et al. 2010 & 3 & 22 & 19 & 28 & $7.8 \%$ & $0.20[0.07,0.59]$ & & & & \\
\hline $\begin{array}{l}\text { Khorsandi M et al. } 2016 \\
\text { Ko WJ et al. } 2002\end{array}$ & $\begin{array}{r}1 \\
25\end{array}$ & $\begin{array}{r}5 \\
61\end{array}$ & $\begin{array}{l}2 \\
4\end{array}$ & $\begin{array}{l}10 \\
15\end{array}$ & $\begin{array}{r}2.5 \% \\
10.2 \%\end{array}$ & $\begin{array}{l}1.00[0.12,8.56] \\
1.54[0.63,3.75]\end{array}$ & & & & \\
\hline Loforte A et al. 2014 & 61 & 126 & 64 & 102 & $25.6 \%$ & $0.77[0.61,0.98]$ & & & & \\
\hline Mikus E et al. 2013 & 3 & 6 & 6 & 8 & $10.2 \%$ & $0.67[0.27,1.63]$ & & & & \\
\hline Ranney DN et al. 2017 & 0 & 95 & 4 & 36 & $1.4 \%$ & $0.04[0.00,0.78]$ & 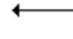 & & & \\
\hline Saeed D et al. 2014 & 11 & 25 & 12 & 12 & $19.9 \%$ & $0.46[0.29,0.72]$ & & $\longrightarrow$ & & \\
\hline Total $(95 \% \mathrm{Cl})$ & & 429 & & 270 & $100.0 \%$ & $0.65[0.46,0.93]$ & & & & \\
\hline Total events & 141 & & 140 & & & & & & & \\
\hline $\begin{array}{l}\text { Heterogeneity: } \mathrm{Tau}^{2}=0.11 \\
\text { Test for overall effect: } \mathrm{Z}=\end{array}$ & $\begin{array}{l}1 ; \mathrm{Chi}^{2}=16 \\
2.35(\mathrm{P}=0 .\end{array}$ & 38, $d f=7$ & $7(P=0.0$ & 2); $1^{2}=5$ & $59 \%$ & & 0.01 & Favours Peripheral ECMO & 1 Favours Central ECMO & 100 \\
\hline \multicolumn{3}{|c|}{ Peripheral ECMO } & \multicolumn{2}{|c|}{ Central ECMO } & \multirow{2}{*}{\multicolumn{2}{|c|}{$\begin{array}{cc} & \text { Risk Ratio } \\
\text { I Weight IV, Random, } 95 \% \mathrm{CI}\end{array}$}} & \multirow{2}{*}{\multicolumn{4}{|c|}{$\begin{array}{c}\text { Risk Ratio } \\
\text { IV, Random, 95\% CI } \\
\end{array}$}} \\
\hline Study or Subgroup & Events & Total & Events & Total & & & & & & \\
\hline Khorsandi M et al. 2016 & 1 & 5 & 2 & 10 & $1.4 \%$ & $6 \quad 1.00[0.12,8.56]$ & & & & \\
\hline Loforte A et al. 2014 & 55 & 126 & 58 & 102 & $94.2 \%$ & $0.77[0.59,1.00]$ & & & & \\
\hline Mikus E et al. 2013 & 3 & 6 & 3 & 8 & $4.4 \%$ & $1.33[0.40,4.43]$ & & & & \\
\hline Total $(95 \% \mathrm{Cl})$ & & 137 & & 120 & $100.0 \%$ & $0.79[0.61,1.02]$ & & & & \\
\hline Total events & 59 & & 63 & & & & & & & \\
\hline $\begin{array}{l}\text { Heterogeneity: } \operatorname{Tau}^{2}=0 \text {. } \\
\text { Test for overall effect: } Z\end{array}$ & $\begin{array}{l}00 ; \mathrm{Chi}^{2}=0 \\
=1.83(\mathrm{P}=\end{array}$ & $\begin{array}{l}82, \mathrm{df}=2 \\
.07)\end{array}$ & $2(P=0.6$ & $6) ; 1^{2}=0$ & & & 0.01 & ${ }^{0.1}{ }^{1}$ & 1 Favours Central ECMO & 100 \\
\hline C & & & & & & & & & & \\
\hline & Peripheral & CMO & Central EC & CMO & & Risk Ratio & & Risk R & Ratio & \\
\hline Study or Subgroup & Events & Total E & Events & Total & Weight & IV, Random, 95\% Cl & & IV, Random & $\mathrm{m}, 95 \% \mathrm{Cl}$ & \\
\hline Kanji HD et al. 2010 & 4 & 22 & 3 & 28 & $23.6 \%$ & $1.70[0.42,6.81]$ & & & 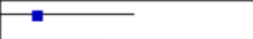 & \\
\hline Khorsandi M et al. 2016 & 1 & 5 & 3 & 10 & $14.2 \%$ & $0.67[0.09,4.89]$ & & & & \\
\hline Ko WJ et al. 2002 & 10 & 61 & 3 & 15 & $29.2 \%$ & $0.82[0.26,2.61]$ & & & & \\
\hline Loforte A et al. 2014 & 13 & 126 & 0 & 102 & $8.0 \%$ & $21.90[1.32,363.97]$ & & & & \\
\hline Saeed D et al. 2014 & 4 & 25 & 3 & 12 & $25.0 \%$ & $0.64[0.17,2.42]$ & & & & \\
\hline Total $(95 \% \mathrm{Cl})$ & & 239 & & 167 & $100.0 \%$ & $1.16[0.49,2.70]$ & & & & \\
\hline Total events & 32 & & 12 & & & & & & & \\
\hline $\begin{array}{l}\text { Heterogeneity: } \operatorname{Tau}^{2}=0.29 \\
\text { Test for overall effect: } Z=\end{array}$ & $\begin{array}{l}9 ; \mathrm{Chi}^{2}=5.8 \\
0.33(\mathrm{P}=0 .\end{array}$ & , df $=4$ & $(P=0.21)$ & $; 1^{2}=32$ & & & 0.01 & Favours Peripheral ECMO & Favours Central ECMO & 100 \\
\hline D & & & & & & & & & & \\
\hline & Periphera & ECMO & Central E & ECMO & & Risk Ratio & & Risk R & Ratio & \\
\hline Study or Subgroup & Events & Total & Events & Total & Weight & IV, Random, $95 \% \mathrm{Cl}$ & & IV, Random & $\mathrm{m}, 95 \% \mathrm{Cl}$ & \\
\hline Kanji HD et al. 2010 & 2 & 22 & 8 & 28 & $38.2 \%$ & $0.32[0.07,1.35]$ & & $\sqrt{-2}$ & - & \\
\hline Khorsandi M et al. 2016 & 0 & 5 & 1 & 10 & $9.7 \%$ & $0.61[0.03,12.80]$ & & & & \\
\hline Mikus E et al. 2013 & 3 & 6 & 3 & 8 & $52.1 \%$ & $1.33[0.40,4.43]$ & & & - & \\
\hline Total $(95 \% \mathrm{Cl})$ & & 33 & & 46 & $100.0 \%$ & $0.71[0.27,1.88]$ & & & & \\
\hline Total events & 5 & & 12 & & & & & & & \\
\hline $\begin{array}{l}\text { Heterogeneity: } \operatorname{Tau}^{2}=0.0 \\
\text { Test for overall effect: } \mathbf{Z}= \\
\text { E }\end{array}$ & $\begin{array}{l}09 ; \mathrm{Chi}^{2}=2 \\
=0.68(\mathrm{P}=\mathrm{C}\end{array}$ & $\begin{array}{l}25, \mathrm{df}=2 \\
50)\end{array}$ & $2(P=0.32$ & 2); $\left.\right|^{2}=1$ & $11 \%$ & & 0.01 & Favours Peripheral ECMO & $\frac{10}{\text { Favours Central ECMO }}$ & 100 \\
\hline
\end{tabular}

Figure 2. Forest plot of the comparison between peripheral and central VA-ECMO in regard to the risk of cerebrovascular events (A), bleeding requiring reoperation (B), continuous veno-venous hemofiltration (C), limb complications (D) and sepsis (E). Risk Ratios (RR) and 95\% Confidence Intervals (CIs); IV, inverse variance. 


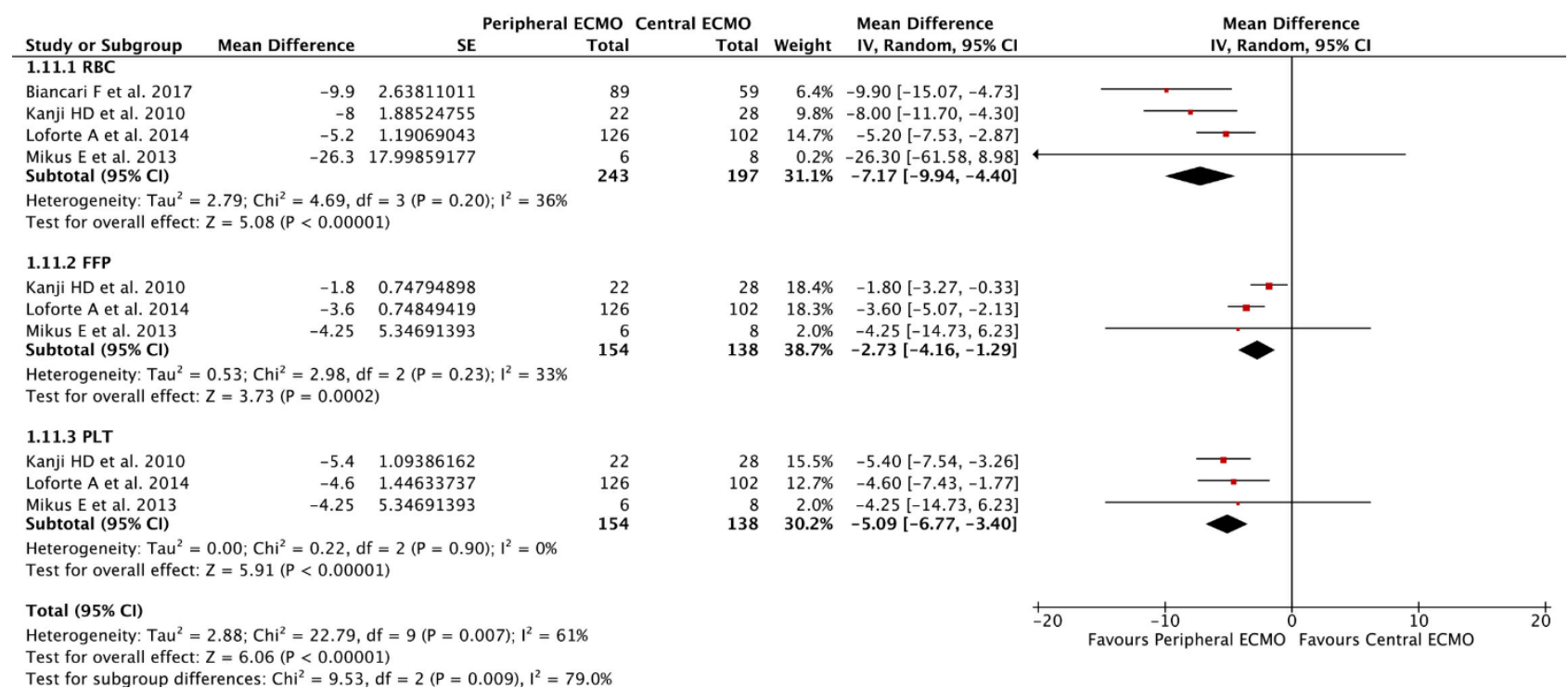

Figure 3. Forest plot of the comparison between peripheral and central VA-ECMO in regard to the risk of transfusions of red blood cells (RBC), fresh frozen plasma (FFP) and platelets (PLT). Risk Ratios (RR) and 95\% Confidence Intervals (CIs); IV, inverse variance.

\section{Comment}

To the best of our knowledge, the current meta-analysis represents the first attempt to address the difference in in-hospital outcomes between central and peripheral cannulation in patients supported with VA-ECMO. The published articles analyzed, enrolling nearly 1,700 patients, have been strictly selected confirming that, although being a relevant and a greatly debated issue, the data available on this topic are very limited. Our main findings were, first, that among patients with PCS and non-PCS, no differences in 30 day in-hospital mortality were observed between peripheral and central cannulation. This was also confirmed in a subgroup analysis including only PCS cases and according to type of surgery. Second, there was no difference between the two VA-ECMO configurations with respect to $\mathrm{CVE}$, sepsis, acute renal injury requiring dialysis and, of particular note, in limb complications. Finally, central cannulation had increased surgical revision for bleeding, as well as use of CVVH, and a higher rate of transfusion of blood products.

ECMO use is increasing, represents a resource-consuming treatment, and, in many cases, is seen as a last resort for patients who, otherwise, would inevitably die [1, 2, 23-25]. PCS was the most frequent 
indication for VA-ECMO implantation in the United States until 2011 [23-25], and the current European Society of Cardiology guidelines recommend ECMO (class of recommendation IIb, level of evidence C) for the management of cardiogenic shock in ST-elevation myocardial infarction [26]. However, despite growing worldwide experience, in-hospital outcomes have not shown substantial progress $[2,27]$. Further, little is known about the cost-benefit ratio in these patients, especially in adult population [28].

PCS and non-PCS are the clinical scenarios where central and peripheral cannulation strategies may both be deployed routinely. The location of ECMO implantation for PCS is mostly represented by the operating theater at the time of surgery (Table 1). In PCS, VA-ECMO is frequently needed for failure from cardiopulmonary bypass weaning and, usually, a central configuration can be easily instituted utilizing the cannulas already in place for previous cardiopulmonary bypass. A peripheral strategy using the femoral, or less frequently axillary or subclavian [10, 29], artery and femoral or jugular vein can be performed with percutaneous access $[4,9]$ or by surgical cut-down or ministernotomy. Advantages of the axillary cannulation include less atherosclerosis, less differential hypoxemia, and greater mobility on ECMO $[3,29]$. The disadvantage is that it requires a cut-down technique which may not be feasible in emergency circumstances. Details on cannulation strategy in order to establish a proper ECMO flow have been reported elsewhere [3]. Central and peripheral cannulation carry advantages and disadvantages (Table 2): the central one directs an antegrade flow stream into the aorta with better cardiac unloading. The peripheral technique is a faster, particularly with regards to femoral arterial access, and less invasive technique for ECMO institution, concomitantly allowing sternal closure, which may be beneficial in terms of bleeding and infectious complications. In PCS, central VA-ECMO may also be instituted with the chest closed -- the ECMO cannulas may be tunneled to exit at the subxyphoid region (Figure 4A) -- allowing patient extubation and mobilization after surgery in case of prolonged support or bridge to more advanced therapies. However, the course of the cannulae, through the mediastinum towards the subxyphoid exit points, may provoke cardiac compression during a weaning attempt. Therefore, in case of expected short 
temporary support and expeditious cardiac recovery, cranial exit ports might be also considered by extending the upper incision with a Y towards the head (Figure 4B). This approach, avoiding cardiac compression by the cannulas, allows an unrestricted cardiac loading during ECMO weaning making the evaluation of hemodynamics more reliable. 


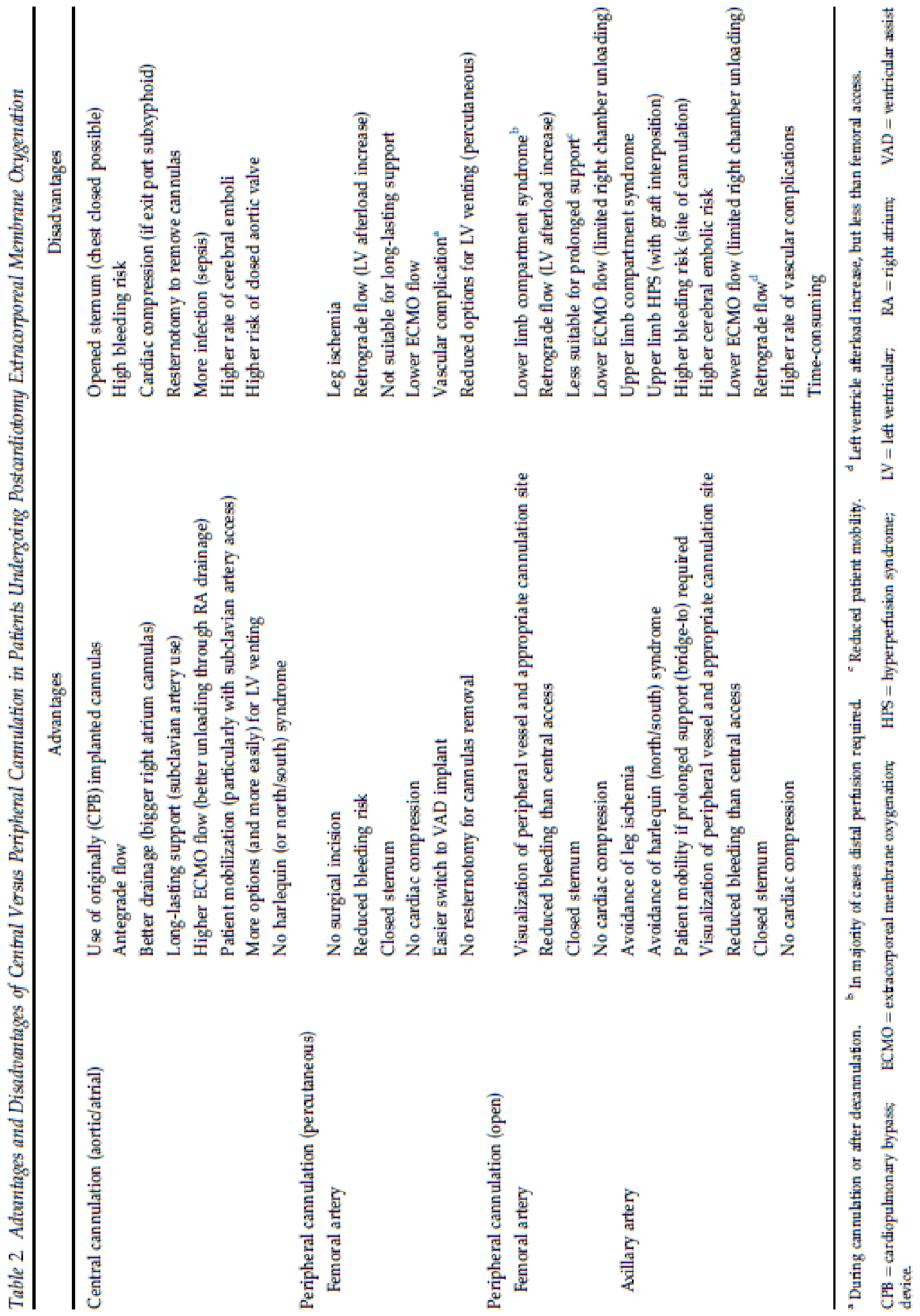




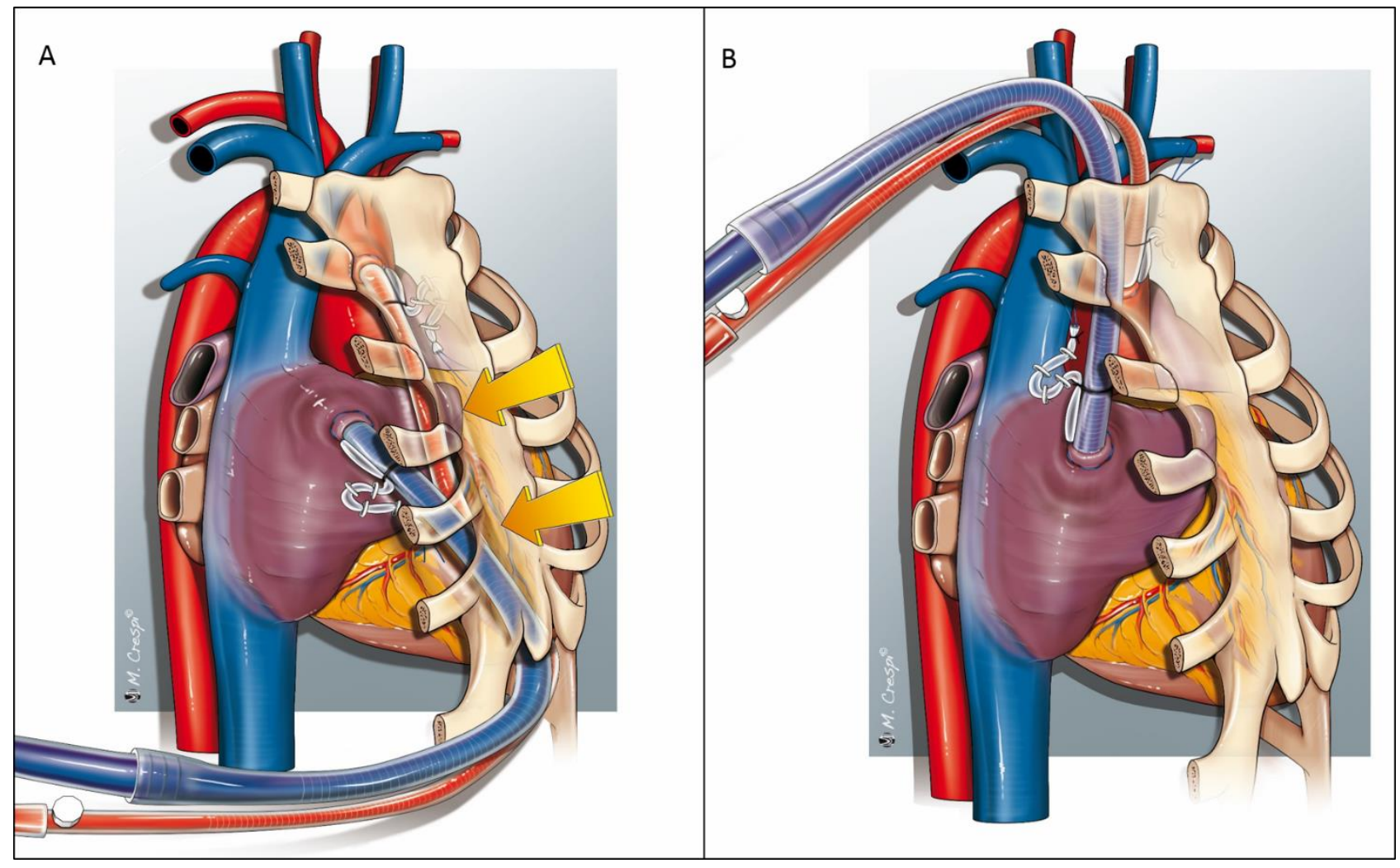

Figure 4. Close chest central VA-ECMO achieved through the subxyphoid (A) and jugular (B) exit sites.

In this meta-analysis, the peripheral cannulation was the cannulation strategy most commonly adopted $(57.7 \%)$ and, notably, in some series was the only one [30-32]. Whenever performed, the open-access was chosen in the majority of the cases [9, 30-32].

The evaluation of hemodynamic performance of VA-ECMO according to cannulation strategy is lacking in the literature. Saeed and colleagues [8] compared the immediate changes in hemodynamics, arterial blood gas value, and end-organ function of patients on either peripheral ECMO or central ECMO support with no particular advantage of one cannulation technique over the other. The lactate levels during ECMO support have been shown to have a predictive role in mortality [33, 34], especially whenever the serial lactate measurements and lactate clearance are evaluated [15]. The majority of the studies included in this meta-analysis reported the differences in the lactate levels between survivors and non-survivors. Similarly, Kanji and colleagues [7] showed no differences in 
the peripheral and central cannulations in the mean peak lactate level as a marker for the end-organ and limb perfusion. Of note, in a series of 517 patients reported by Rastan and colleagues [4], a worse survival (13.9\% vs $22.4 \%$ ) occurred in those who received a percutaneous femoral vein approach (23\%) suggesting a suboptimal venous drainage and compromised ECMO flow as opposed to central right atrial drainage.

LV unloading is another major issue during VA-ECMO [35]. LV dilatation due to lack of contractility, high afterload and retrograde flow towards the aortic valve during VA-ECMO requires careful monitoring and, sometimes, counteractions because of the risk of delayed myocardial recovery as well LV thrombus and pulmonary edema [35]. Six out of 17 studies (Table 1) addressed the importance of LV venting during PCS although it was adopted in the minority of patients. Central VA-ECMO, despite better right heart drainage and an antegrade ECMO flow, may still face insufficient LV unloading. Central ECMO may enhance additional catheter/cannula placement through direct cannulation of vessels or chambers, whereas peripheral configuration, without open chest, may call for alternative solutions [35]. The appropriate or most effective methods for LV venting is still controversial. Intra-aortic balloon counterpulsation (IABP), although controversial [35, 36], may play a role in LV unloading [37]. In some PCS series, the lack of IABP use was linked to a trend to reduced survival $[4,38]$ whereas in other experiences no difference among survivors and non-survivors was also found $[13,16,31]$. Alternative approach in LV unloading, like Impella® (ABIOMED Inc, Danvers, Massachusets), pulmonary artery venting, or other methods, have been also reported [35, 39]. The role, effects, and interaction between various LV venting and central or peripheral ECMO configuration are still unknown and warrant further investigations.

Complications in patients on VA-ECMO are frequent and strongly affect the ultimate outcome [2, 4]. The results of this meta-analysis favor peripheral VA-ECMO in terms of reoperation for bleeding and number of transfusions of red blood cells, fresh frozen plasma and platelets. Bleeding, transfusion and revision for bleeding remain major problems in ECMO patients, and although these may also occur in the peripheral configuration [4], the severity of these complications in central VA-ECMO 
tends to be worse. Open sternotomy to avoid tamponade and right ventricle compression as well as to allow cardiac edema to resolve, the use of cannulae inserted during the previous cardiopulmonary bypass course, and avoiding limb ischemia due to femoral artery cannulation, are the major reasons to choose central cannulation. However, this meta-analysis showed that the risk of limb ischemia and vascular access complications were comparable between the two techniques of cannulation. Small cannula size, distal perfusion cannulae [40] and insertion of a vascular graft are commonly advocated $[4,5,7]$ to avoid such a complication. In relation to the peripheral approach, open cannulation appears to be associated with fewer complications than full percutaneous access [9, 31]. In this meta-analysis, 10 out of 15 studies report on the use of distal perfusion cannula and in only three reports $[4,9,10]$ it was adopted in less than $30 \%$ of the patients. Peripheral complications in central VA-ECMO may include embolic phenomena [7], although the use of vasoconstrictors in these hypovolemic and vasoplegic patients may also play a role. Loforte and colleagues [6] showed that central cannulation in PCS resulted in increased bleeding and CVVH rates compared to peripheral access $(62.7 \% \mathrm{v}$ $48.4 \%$, and $56.8 \% \mathrm{v} 43.6 \%$, respectively). The latter, also confirmed by our analysis, may suggest the high rate of acute volume-depletion events and need for transfusion in the central setting that led to acute renal injury. Kanji et al. [7] investigated the incidence of limb ischemia, perfusion (peak lactate levels), CVE, thrombus and sepsis in the peripheral versus central ECMO over 5 years showing no difference. Of note, the authors report $11 \%$ with limb ischemia in the central cannulation cohort. High rate of neurologic complication was observed in the series of Ko and colleagues [9] treated with open femoral ECMO probably due to the pre-ECMO clinical condition of the patients. The use and impact of axillary or subclavian artery cannulation, which should provide several advantages by allowing a "pseudo-central" flow as compared to femoral artery or ascending aorta cannulation, has been recently investigated [10,41, 42]. A trend towards more frequent implant in the operating room as compared to aorta or femoral artery $(93.8 \%, 80.6 \%$, and $32.9 \%$, respectively), a significant higher rate of vascular complication (particularly fasciotomy and amputation) and bleeding at the cannulation site $(37.5 \%, 30.6 \%$, and $13.9 \%$, respectively) has been recently reported 
[10]. A trend, but not significant, towards higher incidence of CVEs was also observed (18.8\%, $16.7 \%$, and $12.7 \%$, respectively) [10]. The actual incidence, and related techniques to avoid or reduce upper limb hyperperfusion syndrome, was not described [43, 44]. Today, the use of the axillary or subclavian arteries appears therefore advisable in patients with inaccessible femoral arteries or for patients in whom prolonged support (with need of patient awakening, endotracheal extubation and mobility) is expected. However, this should take into account the potentially higher risk of complications as compared with central or femoral artery cannulation [10].

The small number of patients and the retrospective nature of the studies included represent the major limitations of this meta-analysis. Only four studies reported the outcomes of interest in non-PCS [6, $8,10,15]$ and were deliberately included, although addressing a different patient population. This potential selection bias however, did not influence the primary endpoint as shown in the further subgroup analysis including only PCS patients. We also acknowledge the lack of some critical information, e.g. the timing of ECMO starting and distal perfusion and the weaning protocols (please see appendix figure 5, 6 and table 4). Finally, all the analysis were made according to the initial cannulation strategy and the deviations from the planned cannulation strategy ECMO (because of flows, need of adequate LV venting and limb complication) or any changes were not considered.

In conclusion, from the available data in the literature, central versus peripheral access for VA-ECMO in PCS or non-PCS did not show any significant difference in relation to in-hospital survival. Peripheral access was associated with reduced bleeding, transfusion and CVVH rates. No other substantial differences were found regarding other ECMO-related complications. Due to the paucity and limitation of available data, however, further investigations are required to elucidate the riskbenefit profiles of central and peripheral accesses in VA-ECMO. 


\section{References}

1. Lawler PR, Silver DA, Scirica BM, Couper GS, Weinhouse GL, Camp PC, Jr. Extracorporeal membrane oxygenation in adults with cardiogenic shock. Circulation 2015;131:676-680.

2. Whitman GJ. Extracorporeal membrane oxygenation for the treatment of postcardiotomy shock. The Journal of thoracic and cardiovascular surgery 2017;153:95-101.

3. Sorokin V, MacLaren G, Vidanapathirana PC, Delnoij T, Lorusso R. Choosing the appropriate configuration and cannulation strategies for extracorporeal membrane oxygenation: The potential dynamic process of organ support and importance of hybrid modes. European journal of heart failure 2017;19:75-83.

4. Rastan AJ, Dege A, Mohr M et al. Early and late outcomes of 517 consecutive adult patients treated with extracorporeal membrane oxygenation for refractory postcardiotomy cardiogenic shock. The Journal of thoracic and cardiovascular surgery 2010;139:302-311.

5. Raffa GM, Gelsomino S, Sluijpers N et al. In-hospital outcome of post-cardiotomy extracorporeal life support in adult patients: The 2007-2017 maastricht experience. Critical care and resuscitation : journal of the Australasian Academy of Critical Care Medicine 2017;19:53-61.

6. Loforte A, Marinelli G, Musumeci F et al. Extracorporeal membrane oxygenation support in refractory cardiogenic shock: Treatment strategies and analysis of risk factors. Artificial organs 2014;38:E129-141.

7. Kanji HD, Schulze CJ, Oreopoulos A, Lehr EJ, Wang W, MacArthur RM. Peripheral versus central cannulation for extracorporeal membrane oxygenation: A comparison of limb ischemia and transfusion requirements. The Thoracic and cardiovascular surgeon 2010;58:459-462.

8. Saeed D, Stosik H, Islamovic M et al. Femoro-femoral versus atrio-aortic extracorporeal membrane oxygenation: Selecting the ideal cannulation technique. Artificial organs 2014;38:549555. 
9. Ko WJ, Lin CY, Chen RJ, Wang SS, Lin FY, Chen YS. Extracorporeal membrane oxygenation support for adult postcardiotomy cardiogenic shock. The Annals of thoracic surgery 2002;73:538-545.

10. Ranney DN, Benrashid E, Meza JM et al. Central cannulation as a viable alternative to peripheral cannulation in extracorporeal membrane oxygenation. Seminars in thoracic and cardiovascular surgery 2017;29:188-195.

11. Khorsandi M, Dougherty S, Bouamra O et al. Extra-corporeal membrane oxygenation for refractory cardiogenic shock after adult cardiac surgery: A systematic review and meta-analysis. Journal of cardiothoracic surgery 2017;12:55.

12. Biancari F, Dalen M, Perrotti A et al. Venoarterial extracorporeal membrane oxygenation after coronary artery bypass grafting: Results of a multicenter study. International journal of cardiology 2017;241:109-114.

13. Pokersnik JA, Buda T, Bashour CA, Gonzalez-Stawinski GV. Have changes in ecmo technology impacted outcomes in adult patients developing postcardiotomy cardiogenic shock? Journal of cardiac surgery 2012;27:246-252.

14. Mikus E, Tripodi A, Calvi S, Giglio MD, Cavallucci A, Lamarra M. Centrimag venoarterial extracorporeal membrane oxygenation support as treatment for patients with refractory postcardiotomy cardiogenic shock. ASAIO journal (American Society for Artificial Internal Organs:1992) 2013;59:18-23.

15. Slottosch I, Liakopoulos O, Kuhn E et al. Lactate and lactate clearance as valuable tool to evaluate ecmo therapy in cardiogenic shock. Journal of critical care 2017;42:35-41.

16. Unosawa S, Sezai A, Hata M et al. Long-term outcomes of patients undergoing extracorporeal membrane oxygenation for refractory postcardiotomy cardiogenic shock. Surgery today 2013;43:264-270. 
17. Guihaire J, Dang Van S, Rouze S et al. Clinical outcomes in patients after extracorporeal membrane oxygenation support for post-cardiotomy cardiogenic shock: A single-centre experience of 92 cases. Interactive cardiovascular and thoracic surgery 2017;25:363-369.

18. Mazzeffi MA, Sanchez PG, Herr D et al. Outcomes of extracorporeal cardiopulmonary resuscitation for refractory cardiac arrest in adult cardiac surgery patients. The Journal of thoracic and cardiovascular surgery 2016;152:1133-1139.

19. Liberati A, Altman DG, Tetzlaff J et al. The prisma statement for reporting systematic reviews and meta-analyses of studies that evaluate health care interventions: Explanation and elaboration. Annals of internal medicine 2009;151:W65-94.

20. Sterne JA, Hernan MA, Reeves BC et al. Robins-i: A tool for assessing risk of bias in nonrandomised studies of interventions. BMJ 2016;355:i4919.

21. Higgins JP, Thompson SG, Deeks JJ, Altman DG. Measuring inconsistency in meta-analyses. BMJ 2003;327:557-560.

22. Thorlund K, Wetterslev J, Awad T, Thabane L, Gluud C. Comparison of statistical inferences from the dersimonian-laird and alternative random-effects model meta-analyses - an empirical assessment of 920 cochrane primary outcome meta-analyses. Research synthesis methods 2011;2:238-253.

23. Stretch R, Sauer CM, Yuh DD, Bonde P. National trends in the utilization of short-term mechanical circulatory support: Incidence, outcomes, and cost analysis. Journal of the American College of Cardiology 2014;64:1407-1415.

24. McCarthy FH, McDermott KM, Kini V et al. Trends in u.S. Extracorporeal membrane oxygenation use and outcomes: 2002-2012. Seminars in thoracic and cardiovascular surgery $2015 ; 27: 81-88$.

25. Karagiannidis C, Brodie D, Strassmann S, et al. Extracorporeal membrane oxygenation: evolving epidemiology and mortality. Intensive Care Med. 2016;42:889-896. 
26. Ibanez B, James S, Agewall S et al. 2017 ESC guidelines for the management of acute myocardial infarction in patients presenting with st-segment elevation: The task force for the management of acute myocardial infarction in patients presenting with ST-segment elevation of the European Society of Cardiology (ESC). European heart journal 2017. doi: 10.1093/eurheartj/ehx393 27. Chen SW, Tsai FC, Lin YS et al. Long-term outcomes of extracorporeal membrane oxygenation support for postcardiotomy shock. The Journal of thoracic and cardiovascular surgery 2017; 154:469-477 e462.

28. Maxwell BG, Powers AJ, Sheikh AY, Lee PH, Lobato RL, Wong JK. Resource use trends in extracorporeal membrane oxygenation in adults: An analysis of the nationwide inpatient sample 1998-2009. The Journal of thoracic and cardiovascular surgery 2014;148:416-421.

29. Javidfar J, Brodie D, Costa J, et al. Subclavian artery cannulation for venoarterial extracorporeal membrane oxygenation. ASAIO J. 2012;58:494-8.

30. Hsu PS, Chen JL, Hong GJ et al. Extracorporeal membrane oxygenation for refractory cardiogenic shock after cardiac surgery: Predictors of early mortality and outcome from 51 adult patients. European journal of cardio-thoracic surgery: official journal of the European Association for Cardio-thoracic Surgery 2010;37:328-333.

31. Slottosch I, Liakopoulos O, Kuhn E et al. Outcomes after peripheral extracorporeal membrane oxygenation therapy for postcardiotomy cardiogenic shock: A single-center experience. The Journal of surgical research 2013;181:e47-55.

32. Ariyaratnam P, McLean LA, Cale AR, Loubani M. Extra-corporeal membrane oxygenation for the post-cardiotomy patient. Heart failure reviews 2014;19:717-725.

33. Li CL, Wang H, Jia M, Ma N, Meng X, Hou XT. The early dynamic behavior of lactate is linked to mortality in postcardiotomy patients with extracorporeal membrane oxygenation support: A retrospective observational study. The Journal of thoracic and cardiovascular surgery 2015;149:1445-1450. 
34. Rigamonti F, Montecucco F, Boroli F et al. The peak of blood lactate during the first $24 \mathrm{~h}$ predicts mortality in acute coronary syndrome patients under extracorporeal membrane oxygenation. International journal of cardiology 2016;221:741-745.

35. Meani P, Gelsomino S, Natour E et al. Modalities and effects of left ventricle unloading on extracorporeal life support: A review of the current literature. European journal of heart failure 2017;19:84-91.

36. Cheng R, Hachamovitch R, Makkar R et al. Lack of survival benefit found with use of intraaortic balloon pump in extracorporeal membrane oxygenation: A pooled experience of 1517 patients. The Journal of invasive cardiology 2015;27:453-458.

37. Santise G, Panarello G, Ruperto C, et al. Extracorporeal membrane oxygenation for graft failure after heart transplantation: a multidisciplinary approach to maximize weaning rate. Int $\mathrm{J}$ Artif Organs. 2014;37:706-14.

38. Smedira NG, Blackstone EH. Postcardiotomy mechanical support: Risk factors and outcomes. The Annals of thoracic surgery 2001;71:S60-66.

39. Fouilloux V, Lebrun L, Mace L, Kreitmann B. Extracorporeal membranous oxygenation and left atrial decompression: A fast and minimally invasive approach. The Annals of thoracic surgery 2011;91:1996-1997.

40. Juo YY, Skancke M, Sanaiha Y, Mantha A, Jimenez JC, Benharash P. Efficacy of distal perfusion cannulae in preventing limb ischemia during extracorporeal membrane oxygenation: A systematic review and meta-analysis. Artificial organs 2017;41:E263-e273.

41. Chicotka S, Rosenzweig EB, Brodie D, Bacchetta M. The "Central Sport Model": Extracorporeal Membrane Oxygenation Using the Innominate Artery for Smaller Patients as Bridge to Lung Transplantation. ASAIO J. 2017;63:e39-e44.

42. Biscotti M, Gannon WD, Agerstrand C, et al. Awake Extracorporeal Membrane Oxygenation as Bridge to Lung Transplantation: A 9-Year Experience. Ann Thorac Surg. 2017;104:412-419. 
43. Biscotti M, Bacchetta M. The "sport model":extracorporeal membrane oxygenation using the subclavian artery. Ann Thorac Surg. 2014;98:1487-9.

44. Navia JL, Atik FA, Beyer EA, Ruda Vega P. Extracorporeal membrane oxygenation with right axillary artery perfusion. Ann Thorac Surg. 2005;79:2163-5.

45. Zhao Y, Xing J, Du Z, Liu F, Jia M, Hou X. Extracorporeal cardiopulmonary resuscitation for adult patients who underwent post-cardiac surgery. Eur J Med Res. 2015;20:83.

46. Russo CF, Cannata A, Lanfranconi M, et al. Veno-arterial extracorporeal membrane oxygenation using Levitronix centrifugal pump as bridge to decision for refractory cardiogenic shock. $\mathrm{J}$ Thorac Cardiovasc Surg. 2010;140:1416-21. 


\section{CHAPTER 5}

In-Hospital Outcomes after Emergency or Prophylactic Veno-Arterial Extracorporeal Membrane Oxygenation during Transcatheter Aortic Valve Implantation:

A Comprehensive Review of the Literature

Raffa GM, Kowalewski M, Meani P, Follis F, Martucci G, Arcadipane A, Pilato M, Maessen J, Lorusso R.

Perfusion. 2019 Jul;34(5):354-363 


\section{Abstract}

Veno-arterial extracorporeal membrane oxygenation (V-A ECMO) has been used to deal with lifethreatening complications as well as back-up or active cardiovascular support during high-risk procedures in patients undergoing transcatheter aortic valve implantation (TAVI). PubMed and Medline electronic databases were searched in order to identify studies with emergency or prophylactic V-A ECMO application in association with TAVI procedures. From November 2012 to November 2017, 14 relevant studies were identified that included 5,115 TAVI patients of whom 102 (2\%) required ECMO (22 prophylactically, 66 as an emergency and 14 without a reported indication). The reason for emergency V-A ECMO institution was detailed in 64 patients: left ventricle free wall rupture $(n=14)$, haemodynamic instability $(n=12)$, ventricular arrhythmias $(n=7)$, aortic annulus rupture $(n=6)$, coronary obstruction $(n=6)$, low left ventricular output (ejection fraction<35\%) $(n=5)$, uncontrollable bleeding $(n=5)$, severe aortic regurgitation $(n=4)$, prosthesis embolisation $(n=3)$, aortic dissection $(n=1)$ and respiratory failure $(n=1)$. Femoral arterial and vein cannulation was the most common access technique for V-A ECMO institution. Major bleeding $(n=7)$ and vascular access complications $(n=7)$ were reported after ECMO institution. The overall in-hospital survival was $73 \%$ (61\% in the emergency vs $100 \%$ in the prophylactic group).

V-A ECMO support should be available at any centre performing TAVI and provides effective mechanical circulatory support in an emergency setting. We present an algorithm to aid decisions about prophylactic circulatory assistance with ECMO and it should form part of the heart team discussion before a TAVI procedure is undertaken. 


\section{Introduction}

Mechanical circulatory support (MCS) devices such as cardiopulmonary bypass, intraaortic balloon pump, TandemHeart device (CardiacAssist, Inc., Pittsburgh, PA, USA), and the Impella System (Abiomed, Inc., Danvers, MA, USA), have been variably applied in patients with cardiogenic shock and high-risk percutaneous coronary interventions.1 With the development of transcatheter aortic valve implantation (TAVI) technology, MCS has also been advocated in these patients as a prophylactic or emergency strategy for challenging conditions. ${ }^{1,2}$ Compared to the previous results of the first TAVI trial, the need for MCS during such a procedure dropped from $8 \% 3$ to $1 \%$ in the most recent series, ${ }^{4,5}$ reflecting improvements in device technology, increased operator experience, and, most likely, better patient selection, and a lower-risk patient profile. Among temporary artificial support systems, veno-arterial extracorporeal membrane oxygenation (V-A ECMO) has emerged over the past decade as a preferable tool due to expeditious and percutaneous access for cannulation, device miniaturisation and portability, enhanced blood gas exchange during cardiogenic shock, and limited cost compared to other percutaneous circulatory support systems. ${ }^{6,7} \mathrm{~V}$-A ECMO has been used particularly in emergency cases for life-threatening TAVI-related peri-procedural complications such as cardiac tamponade, severe paravalvular regurgitation and cardiogenic shock, aortic valve annulus rupture, ventricular perforation, ventricular arrhythmias, coronary obstruction and prosthesis migration, and persistent impaired contractility impairment after TAVI deployment. ${ }^{5}$ The prophylactic implantation of V-A ECMO has also been applied to achieve haemodynamic stabilisation or cardiac unloading during high-risk TAVI cases. ${ }^{5}$ The actual outcome of TAVI patients undergoing V-A ECMO, however, remains poorly defined. A review of the literature of prophylactic and emergency V-A ECMO during TAVI has been therefore undertaken in order to define the indications and surgical techniques and evaluate the clinical results.

\section{Methods}


Literature search criteria. Selection criteria included studies in English describing clinical series or reports of emergency or prophylactic V-A ECMO institution during or after TAVI procedures. Electronic searches were conducted in PubMed and MEDLINE databases from November 2012 to November 2017. The reference lists of all the retrieved articles were reviewed for further identification of potentially relevant studies. No researchers or sponsors were contacted by the authors to acquire any missing information. To identify all of the relevant studies, we used "transaortic valve implantation" and "TAVI" and "extracorporeal membrane oxygenation" and "ECMO" as free text and thesaurus terms. Eligibility assessment was performed by one reviewer, the first author. The reviewer screened the retrieved records by titles and abstracts, and the full-text publications were reviewed. To avoid duplications, only the most recent study on the topic by the same authors was included in the analysis. Non-peer-reviewed studies and abstracts were excluded. Information was extracted from each article on: (1) the characteristics of the patients (candidates for TAVI requiring prophylactic or emergency V-A ECMO placement), (2) the type of intervention (TAVI procedures), and (3) the outcome measure (weaning rate, complication rate, and survival rate). Articles reporting mechanical circulatory support during TAVI other than V-A ECMO (for example, standard cardiopulmonary bypass, intraaortic balloon pump, and the Impella and TandemHeart devices) were excluded.

Continuous variables are expressed as mean, standard deviation, and range; categorical variables are expressed as absolute values and percentages.

\section{Results}

A total of 21 peer-reviewed publications were retrieved. After removal of not pertinent reports and duplications (references 15 and 19, and 14 and 20, respectively), 14 papers remained (Table 1). ${ }^{1,2,5,8-}$ ${ }^{18}$ Two articles ${ }^{5,16}$ from the same study group were considered because of prophylactic use of V-A ECMO in earlier series ${ }^{16}$ not reported in the latest one5 (thus the emergency V-A ECMO in the 
former $^{16}(\mathrm{n}=9)$ was not included in the overall population of this review). The full texts of the remaining studies were then assessed in detail.

The characteristics of the relevant studies are detailed in Table 1, including 5,115 TAVI patients of whom $102(2 \%)$ had prophylactic $(n=22)$ or emergency $(n=66) \mathrm{V}$-A ECMO during TAVI procedures. In the remaining 14 patients, ${ }^{1}$ the strategy was not detailed. Four of the 14 articles were case reports. ${ }^{10,12,18,20}$

The results of emergency and prophylactic V-A ECMO during TAVI are summarised in Table 2. Demographics were not specified for 18 patients; in the remaining 84 subjects, the average age was $79 \pm 5$ years (range $69-87$ years) and 43 were male.

TAVI technical details. Transfemoral TAVI was the most common approach $(n=40)$ followed by the transapical TAVI $(n=29)$. Transaortic TAVI was applied in two cases. In the remaining cases, data were not specified. The most common type of prostheses used for the TAVI procedure were a SAPIEN valve (Edwards Lifesciences, Irvine, CA, USA) (n=29). Other prostheses included CoreValve (Medtronic, Irvine, CA, USA), Acurate NEO and TA (Symetis SA, Ecublens, Switzerland), Direct Flow Medical (Direct Flow Medical, Inc., Santa Rosa, CA, USA), and JenaValve (JenaValve Technology GmbH, Munich, Germany). 


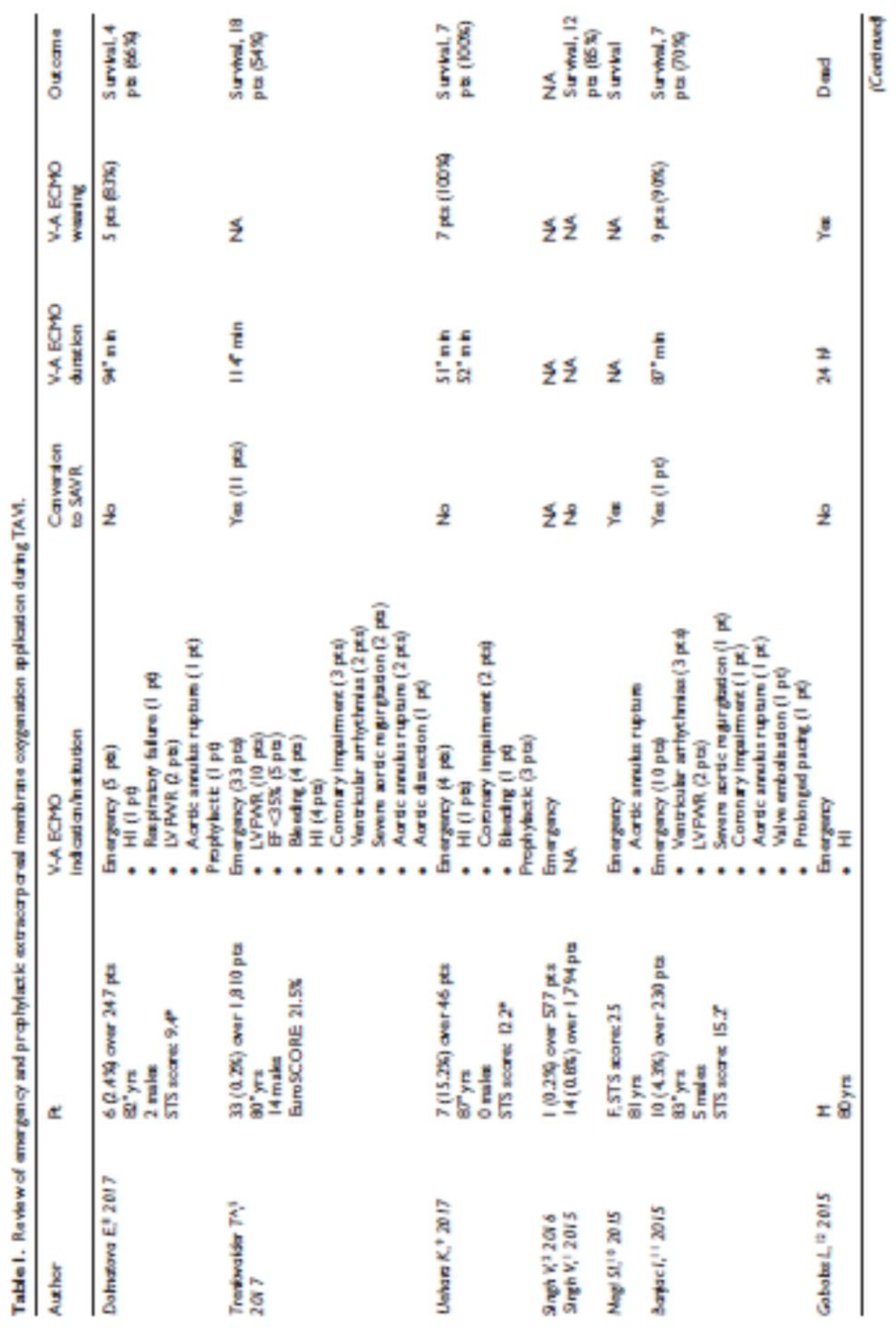




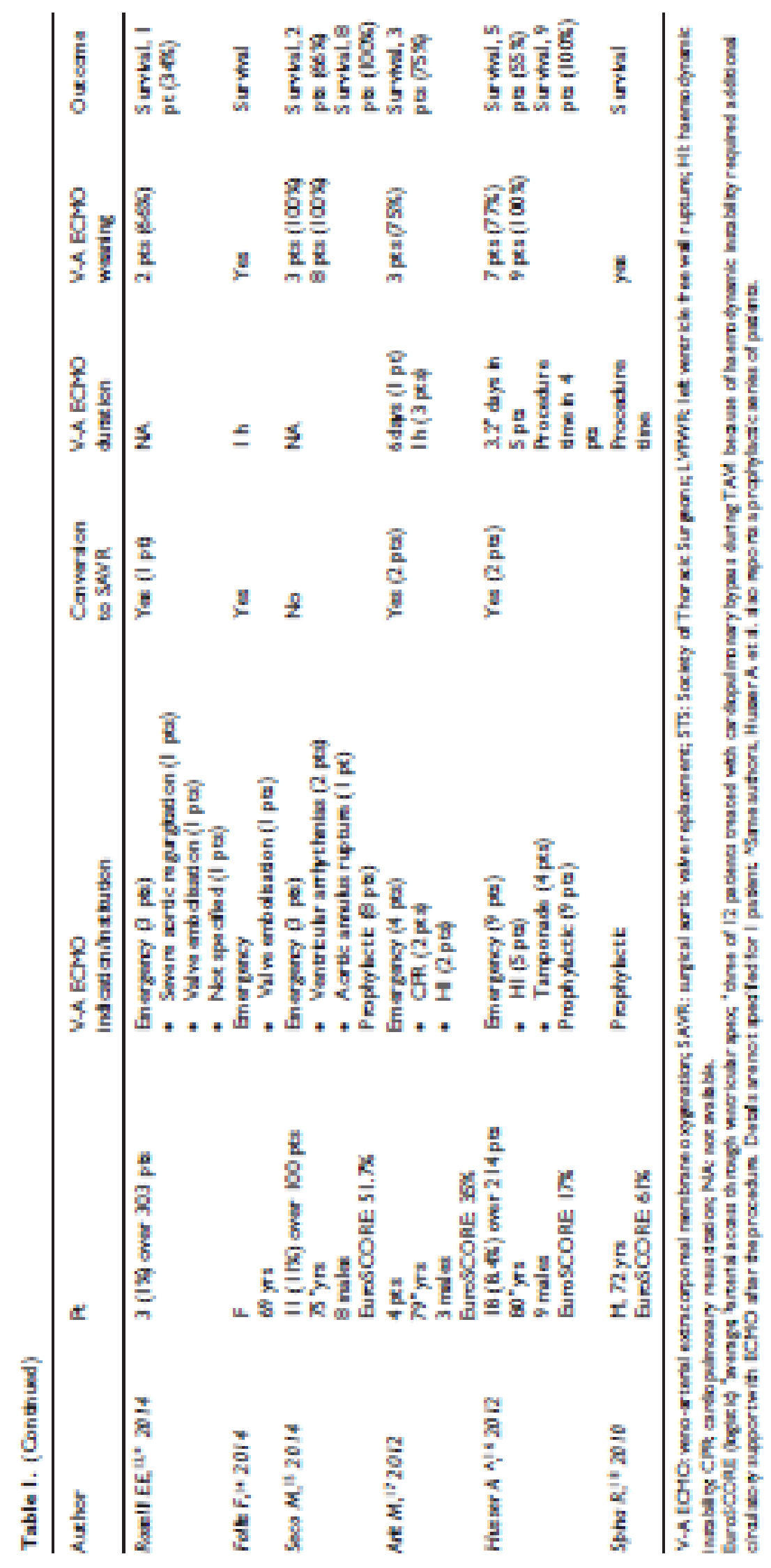

Indication for emergency V-A ECMO institution. The reason for emergency V-A ECMO institution was detailed in 64 patients: left ventricle free wall rupture $(n=14)$, haemodynamic instability $(n=12)$, 
ventricular arrhythmias $(n=7)$, aortic annulus rupture $(n=6)$, coronary obstruction $(n=6)$, low left ventricular output (ejection fraction<35\%) $(n=5)$, uncontrollable bleeding $(n=5)$, severe aortic regurgitation $(n=4)$, prosthesis embolisation $(n=3)$, aortic dissection $(n=1)$ and respiratory failure $(n=1)$.

V-A ECMO technical details. Cannulation details are available for 67 patients. Femoral vein cannulation was used in all cases. Femoral arterial and vein cannulation was the most common access for V-A ECMO implants ( $n=62)$, with a percutaneous approach employed in 54 patients. Subclavian9, $18(\mathrm{n}=2)$ and aortic11 $(\mathrm{n}=2)$ arterial cannulation was also used. In one case, ${ }^{12}$ a transapical approach followed by transaortic valvular positioning of the cannula was used for the patient arterial inflow.

The reported size of the arterial cannulas ranged from 15 Fr. to 19 Fr. and from 19 to 24 Fr for the venous cannulas. The use of distal percutaneous perfusion cannulas in order to prevent lower limb ischaemia was mentioned by two authors and was established in case of an expected prolonged need for V-A ECMO. ${ }^{5,11}$

V-A ECMO as bridge to surgical aortic valve replacement. Conversion to surgical aortic valve replacement in patients on emergency V-A ECMO during TAVI was necessary in 19 patients.

V-A ECMO-related complications. V-A ECMO-related complications during TAVI were detailed in two articles ${ }^{5,15}$ including 44 patients and these were major bleeding $(\mathrm{n}=7)$ and vascular access complications $(n=7)$.

In-hospital survival. The overall survival of patients requiring prophylactic or emergency V-A ECMO during TAVI was $73 \%$ (74 patients out of 102). In cases of emergency V-A ECMO implantation, the survival rate was $61 \%$ (40 patients out of 66 ), whereas the survival rate was $100 \%$ in the prophylactic group. 


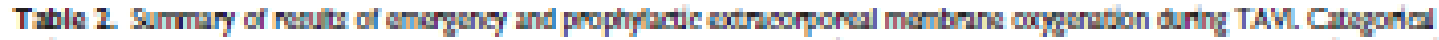

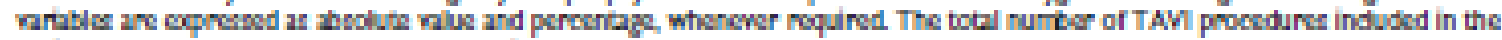

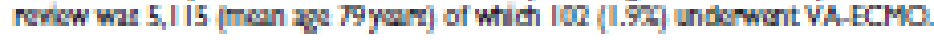

\begin{tabular}{|c|c|}
\hline Y.AECHO dutre TAVI & $n=102(x+)$ \\
\hline \multicolumn{2}{|l|}{ Stratery } \\
\hline - Emeroncy & LEGB TSW \\
\hline - Proptyadie & 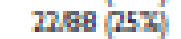 \\
\hline - Not quttird tab & 14 \\
\hline \multicolumn{2}{|l|}{ Gender } \\
\hline F Mla & $43845 \%$ \\
\hline - Futrele & $4184+5 n$ \\
\hline - Not quathd thb & 19 \\
\hline \multicolumn{2}{|l|}{ TAM Approuch } \\
\hline * Trintemen & 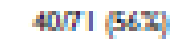 \\
\hline - Trumpial & $29714 x$ \\
\hline - Troncorte & 27109 \\
\hline - Not quthed thb & 31 \\
\hline \multicolumn{2}{|l|}{ Indlation for smergency Y.A ECMO } \\
\hline - LYPWA & 1441717 \\
\hline * Hhemodymik intality & $1244 \mid$ \\
\hline * Vortuarartipthma & $7 M 411 \mathrm{x}$ \\
\hline - Aonte anulur nupure & 6494 \\
\hline * Comar impaimentr & 6494 \\
\hline - Low latt wontrialar output & $504 \mathrm{ax}$ \\
\hline * Eluoding & $54 \mathrm{an}$ \\
\hline * Sware jorte regurjaton & 444 \\
\hline - Frother smboliation & 245 \\
\hline - Aork dimetion & 1475 \\
\hline - Ropintory tilure & $1047 x$ \\
\hline - Not quthind the & 1 \\
\hline \multicolumn{2}{|l|}{ Y.A ECMO latutlon } \\
\hline - Fumonl artry and wh & 624793 \\
\hline - Fontanou zorodi & $54 \mathrm{~g}$ \\
\hline - Not quatiad tib & 35 \\
\hline $\begin{array}{l}\text { Conwrion to Eurgheal AYR in putenta on emmegrey Y-A ECHO } \\
\text { Y.A ECMO-related complioutions }\end{array}$ & 1946 797 \\
\hline * Uhwoitollak Bosthe & 744167 \\
\hline - Vecular acoses complation & $7446 x$ \\
\hline \multicolumn{2}{|l|}{ Survimal } \\
\hline - Omen & $7410207 x$ \\
\hline - Emerong Y.EEHO & 4046417 \\
\hline - Frophytate V-A ECHO & nan $100 \mathrm{~h}$ \\
\hline
\end{tabular}

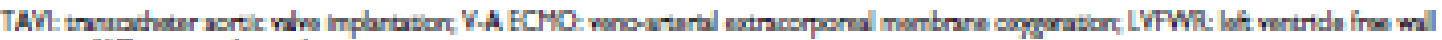

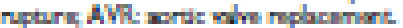

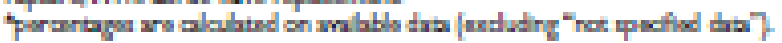

\section{Discussion}

TAVI has become a widely accepted alternative for patients at high surgical risk with severe symptomatic native aortic valve stenosis.21-26 Based on the results of the PARTNER A and B trials, as well as the SURTAVI trial, comparing TAVI to surgical aortic prosthesis implantation, ${ }^{24-26}$ transcatheter procedures continue to increase beyond the populations originally enrolled, shifting from high-risk, frail, and inoperable patients, to intermediate- and low-risk populations. Despite advancements in technology, ${ }^{5}$ TAVI remains a complex procedure that may result in serious complications, especially in centres commencing new clinical programmes. ${ }^{7,22}$ The use of MCS to 
manage such complications or to provide a haemodynamical back-up to enhance TAVI was applied in a marginal number of the treated patients, but is likely to increase if the indications for TAVI are widened. ${ }^{1,2,6}$ Singh et al. analysed 1,794 TAVI procedures from the Nationwide Inpatient Sample in the United States1; MCS devices were used in 190 patients (10.6\%), $14(7.4 \%)$ of which were ECMOs. MCS was associated with a higher mortality rate, more complications, and increased length and cost of hospitalisation. Female gender, acute myocardial infarction, concomitant percutaneous intervention, ventricular fibrillation arrest, transapical access, and cardiogenic shock were the most significant predictors of MCS use during TAVI. Subsequently, the same authors ${ }^{2}$ published a singlecentre experience including 577 TAVIs, with 54 requiring MCS (ECMO in 3 patients). The bleeding and/or vascular complication rate increased to $30 \%$, with in-hospital and 1-year mortality reaching $24 \%$ and $35 \%$, respectively; however, that was lower than reported in the PARTNER trial and the parallel registry (29\% and $49 \%$ at 1 year, respectively). ${ }^{27}$

Among the MCS devices employed during TAVI procedures, cardiopulmonary bypass has been commonly adopted in some series. ${ }^{13,28}$ From the technical standpoint, among the MCS devices, Impella needs the wire in the left ventricle with the aortic prosthesis already deployed before it can be inserted, while TandemHeart requires transeptal puncture and implantation of large cannulas, manoeuvres that are time-consuming and challenging in an emergency situation. ${ }^{29}$ On the other hand, V-A ECMO only needs insertion of femoral arterial and venous cannulas over guidewires already present in the vessels during most TAVI procedures.

V-A ECMO use grew exponentially from 2006 to $201130-35$ and has emerged as a prevalent prophylactic and emergency treatment during TAVI since 2010.18 In this review of more than 5,000 patients, the use of V-A ECMO in the emergency setting (1.3\%) was significantly lower than in previous reports..$^{1,2}$

Previous reports ${ }^{6,7}$ similar to our review identified two subsets of patients where V-A ECMO support was used and characterised by a remarkable difference in the survival rate. The first group included patients placed on V-A-ECMO "pre-emptively" before the procedure, mainly because of 
haemodynamic instability or poor cardiac function, who benefitted from the prophylactic circulatory support during the vagaries of the procedure but subsequently improved rapidly after the aortic stenosis was relieved. In previous series and in our review, they had $100 \%$ survival. On the other hand, the second patient group accounted for those who were placed on V-A ECMO on an emergency basis due to the occurrence of a severe complication and showing a much lower survival rate (approximately 60\%). By the time of ECMO institution, these patients had already suffered from structural damage (coronary occlusion, annular rupture, aortic dissection, valvular malposition, and/or left ventricular disruption after transapical approach) and the increased mortality was probably related to the impact of such conditions. ${ }^{36}$

Severe intraprocedural complications can occur in up to $7.6 \%$ of TAVI procedures. ${ }^{37}$ In many of these circumstances, V-A ECMO can bridge the patients to emergency cardiac operations. In this analysis, free wall rupture was the most common cause of emergency V-A ECMO implantation and may be related to the complicated transapical procedures, aortic annular rupture, or reoperation with cardiac injury during chest re-entry. ${ }^{5,38-40}$ In our review, conversion to surgical aortic valve replacement was necessary in $28 \%$ of patients requiring emergency V-A ECMO after TAVI, mainly due to embolisation of the valve prosthesis into the left ventricle or migration into the left ventricular outflow tract. In cases of femoral V-A ECMO because of severe aortic regurgitation after failed TAVI, careful left ventricle drainage is required. ${ }^{29}$ Despite not specified data and a lack of differentiation in the literature between TAVI- and V-A ECMO-related vascular access complications and bleeding ${ }^{41}$, in the current analysis, both complications occurred in a small portion of ECMO patients, highlighting the relative safety of this strategy even in emergency scenarios.

ECMO availability and technology is not always present in hospitals where TAVI is performed and is even less frequent in those without cardiac surgery facilities. ${ }^{37}$ In addition, the institution of V-A ECMO and its maintenance are costly and resource-intensive endeavours. We estimated (our own unpublished data) that an average short-run V-A ECMO support lasting 1 to 2 hours and in accordance with the elective cases collected in our review would cost approximately 5,000 euros. 
In light of the aforementioned considerations, we believe that this technology should be used sparingly and the indications for prophylactic and standby ECMO should be carefully evaluated ahead of time by the heart team during the preoperative discussion of the patient and carefully considered during the procedure.

The most common scenarios that have led to the prophylactic institution of ECMO were as follows: (1) heart failure requiring hospitalisation and stabilisation pre-TAVI; (2) pre-operative assessment of moderate or severe left and/or right ventricular failure; (3) haemodynamic instability during balloon aortic valvuloplasty performed to improve heart function pre-TAVI; (4) borderline haemodynamics during procedures with central venous pressure/pulmonary capillary wedge pressure $>20 \mathrm{mmHg}$, mean pulmonary artery pressure $>40 \mathrm{mmHg}$, and cardiac index $<2.0$ with no improvement in inotropes; (5) slow recovery from rapid left ventricular pacing during testing of a pacemaker; (6) high vasopressor requirements during general anaesthesia; and (7) concomitant high-risk percutaneous coronary intervention. In addition, the heart team should look for unfavourable patient-related features that may include some hallmarks of structural complications such as coronary ostia close to the annulus, small and calcified annuli, and dilated aortas, in order to have ECMO in a standby mode, especially for institutions without on-site cardiac surgery.

Needless to say, the heart team should be composed by all of the individuals involved in the care of the patient: the referring cardiologist, the interventional cardiologist, the cardiac surgeon, the cardiac anaesthesiologist, the perfusionist, and the OR and cath lab nurses ${ }^{36}$ For centres without an in-house cardiac surgery back-up unit, a remote consultation during the case decision-making and a hub-andspoke network to manage emergency transport and surgery if appropriate might represent an additional asset for patient safety.

Conversely, for standard non-high-risk patients, ECMO standby could be spared, avoiding the inevitable costs of circuit set-up and personnel availability.

Once a patient has been placed on V-A ECMO, the outcome would most probably depend on the original indication. The prophylactic cases are gradually weaned off support after valve implantation 
and relief of aortic stenosis; the emergency cases are transferred directly to the operating room for repair of structural damages.

In addition, there is currently a wide debate about the anaesthesia management during TAVI, with the two main strategies, monitored anaesthesia care and general anaesthesia. Both approaches carry comparable short term clinical outcomes, shortening of length of stay and reduction in costs. ${ }^{42}$ In high-risk TAVI the general anaesthesia is probably the preferred strategy since it may assure a more efficient cardiorespiratory support in case of ECMO institution. ${ }^{43}$

The strategy described can be summarised in an algorithm of indications and patient management (Fig. 1).

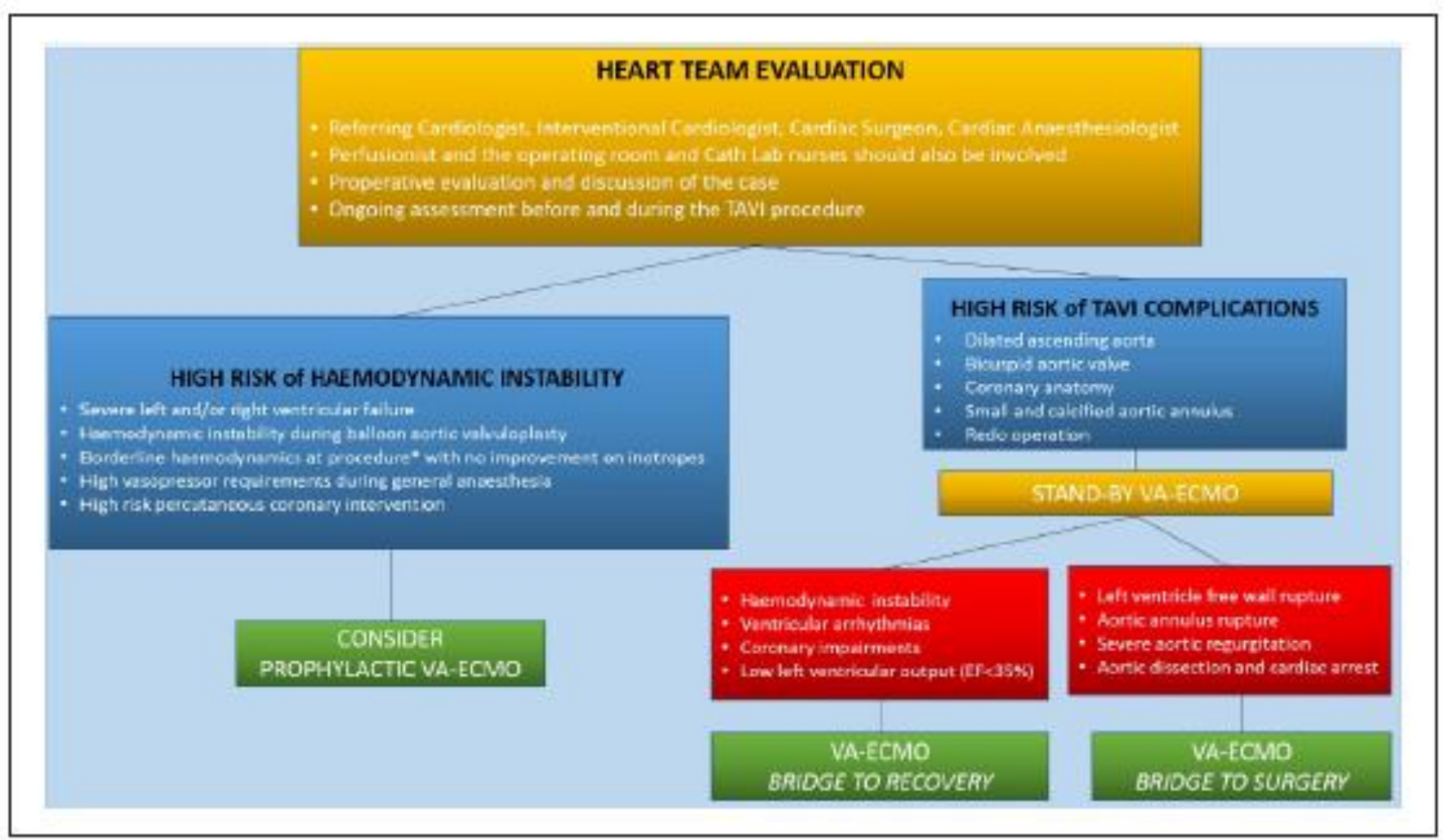

Figure 1. A proposed algorithm of prophylactic and emergency veno-arterial extracorporeal membrane oxygenation (V-A ECMO) during TAVI for institutions without cardiac surgery on site.

"Central venous pressure/pulmonary capillary wedge pressure $>20 \mathrm{mmHg}$, mean pulmonary artery pressure $>40 \mathrm{mmHg}$ and cardlac index $<20$, slow recovery from rapid left ventricular pacing during testing of a pacemaker.

Finally, the practise of institutions performing TAVI without on-site cardiac surgery is questionable, since international guidelines universally recommend cardiac surgery back-up for such procedures. Therefore, V-A ECMO support indicated on a prophylactic or standby mode, should be arranged and available on site or with another hospital ECMO team acting as back-up for rapid intervention. Initial 
TAVI series were characterised by patients with advanced age, inoperable, and severe comorbidities. Accordingly, the application of V-A ECMO has been limited because of the poor prognosis of MCS in these patients. However, increasing experiences and improved technology in temporary cardiocirculatory support have led to more frequent employment even in complicated cases. Indeed, as shown by Stretch et al., ${ }^{32}$ the utilisation of short-term MCS in patients older than 80 increased from $6.2 \%$ in $2004-2007$ to $11.9 \%$ in $2008-2011$. Furthermore, with broadening indications for TAVI for low-risk and younger patients, the lack of V-A ECMO capability or organisation at the time of the procedure20 might become ethically unjustifiable and legally indefensible.

The small number of patients, the retrospective nature of the studies included, and the varying frequency of ECMO use (from $0.2 \%$ to $15 \%$ ) represent the major limitations of this review; thus, the real benefit from V-A ECMO during TAVI should be carefully considered. Indeed, it has to be clearly stated that the subject of V-A ECMO during TAVI is still guided by expert opinion alone and will remain so until randomised trials are available to address its feasibility and safety. Additionally, reports are always slightly behind the current state.

In conclusion, among mechanical circulatory support systems, V-A ECMO is emerging as the optimal temporary cardio-circulatory support with a relative ease of institution in an emergency setting, transport simplicity, and lower cost. Indications for prophylactic or standby V-A ECMO should be taken into consideration during the heart team evaluation, and it should be promptly instituted when major complications occur. 


\section{References}

1. Singh V, Patel SV, Savani C, et al. Mechanical circulatory support devices and transcatheter aortic valve implantation (from the National Inpatient Sample). Am J Cardiol. 2015;116:1574-80.

2. Singh V, Damluji AA, Mendirichaga R, et al. Elective or Emergency Use of Mechanical Circulatory Support Devices During Transcatheter Aortic Valve Replacement. J Interv Cardiol. 2016;29:513-22.

3. Makkar RR, Jilaihawi H, Chakravarty $\mathrm{T}$, et al. Determinants and outcomes of acute transcatheter valve-in-valve therapy or embolization: a study of multiple valve implants in the U.S. PARTNER trial (Placement of AoRTic TraNscathetER Valve Trial Edwards SAPIEN Transcatheter Heart Valve). J Am Coll Cardiol. 2013;62:418-30.

4. Kodali S, Thourani VH, White J, et al. Early clinical and echocardiographic outcomes after SAPIEN 3 transcatheter aortic valve replacement in inoperable, high-risk and intermediate-risk patients with aortic stenosis. Eur Heart J. 2016;37:2252-62.

5. Trenkwalder T, Pellegrini C, Holzamer A, et al. Emergency extracorporeal membrane oxygenation in transcatheter aortic valve implantation: A two-center experience of incidence, outcome and temporal trends from 2010 to 2015. Catheter Cardiovasc Interv. 2017 Nov 11. doi: 10.1002/ccd.27385.

6. Makdisi G, Makdisi PB, Wang IW. New horizons of non-emergent use of extracorporeal membranous oxygenator support. Ann Transl Med. 2016;4:76.

7. Makdisi G, Makdisi PB, Wang IW. Use of extracorporeal membranous oxygenator in transcatheter aortic valve replacement. Ann Transl Med. 2016;4:306.

8. Dolmatova E, Moazzami K, Cocke TP, et al. Extracorporeal membrane oxygenation in transcatheter aortic valve replacement. Asian Cardiovasc Thorac Ann. 2017;25:31-4.

9. Uehara K, Minakata K, Saito N, et al. Use of extracorporeal membrane oxygenation in complicated transcatheter aortic valve replacement. Gen Thorac Cardiovasc Surg. 2017;65:329-36. 
10. Negi SI, Patel J, Patel M, Loyalka P, Kar B, Gregoric I. Successful repair of aortic annulus rupture during transcatheter aortic valve replacement using extracorporeal membrane oxygenation support. Gen Thorac Cardiovasc Surg. 2015;63:514-7.

11. Banjac I, Petrovic M, Akay MH, et al. Extracorporeal Membrane Oxygenation as a Procedural Rescue Strategy for Transcatheter Aortic Valve Replacement Cardiac Complications. ASAIO J. 2016;62:e1-4.

12. Gobolos L, Tsang GM, Curzen N, Calver AL, Ohri SK. Transapical perfusion for peri-arrest salvage during transcutaneous aortic valve implantation. Perfusion. 2015;30:650-2.

13. Roselli EE, Idrees J, Mick S, et al. Emergency use of cardiopulmonary bypass in complicated transcatheter aortic valve replacement: importance of a heart team approach. $\mathrm{J}$ Thorac Cardiovasc Surg. 2014;148:1413-6.

14. Follis F, Gandolfo C, Santise G, et al. Prosthesis embolization into the left ventricle during transcatheter aortic valve implantation. J Cardiovasc Med (Hagerstown). 2016;17 Suppl 2:e191-e2.

15. Seco M, Forrest P, Jackson SA, et al. Extracorporeal membrane oxygenation for very highrisk transcatheter aortic valve implantation. Heart Lung Circ. 2014;23:957-62.

16. Husser O, Holzamer A, Philipp A, et al. Emergency and prophylactic use of miniaturized veno-arterial extracorporeal membrane oxygenation in transcatheter aortic valve implantation. Catheter Cardiovasc Interv. 2013;82:E542-51.

17. Arlt M, Philipp A, Voelkel S, et al. Early experiences with miniaturized extracorporeal lifesupport in the catheterization laboratory. Eur J Cardiothorac Surg. 2012;42:858-63.

18. Spina R, Forrest AP, Adams MR, Wilson MK, $\mathrm{Ng} \mathrm{MK}$, Vallely MP. Veno-arterial extracorporeal membrane oxygenation for high-risk cardiac catheterisation procedures. Heart Lung Circ. 2010;19:736-41.

19. $\mathrm{Yu} \mathrm{Y,} \mathrm{Vallely} \mathrm{M}, \mathrm{Ng} \mathrm{MK}$. Valve-in-valve implantation for aortic annular rupture complicating transcatheter aortic valve replacement (TAVR). J Invasive Cardiol. 2013;25:409-10. 
20. Follis F, D'Ancona G, Santise G, Follis M. TAVI without surgical standby: is history repeating itself? A word of caution. J Thorac Cardiovasc Surg. 2014;147:541-2.

21. Gozdek M, Raffa GM, Suwalski P, et al. Comparative performance of transcatheter aortic valve-in-valve implantation versus conventional surgical redo aortic valve replacement in patients with degenerated aortic valve bioprostheses: systematic review and meta-analysis. Eur J Cardiothorac Surg. 2018;53:495-504.

22. Raffa GM, Malvindi PG, Settepani F, et al. Aortic valve replacement for paraprosthetic leak after transcatheter implantation. J Card Surg. 2012;27:47-51.

23. Gargiulo G, Sannino A, Capodanno D, et al. Transcatheter Aortic Valve Implantation Versus Surgical Aortic Valve Replacement: A Systematic Review and Meta-analysis. Ann Intern Med. 2016;165:334-44.

24. Leon MB, Smith CR, Mack M, et al. Transcatheter aortic-valve implantation for aortic stenosis in patients who cannot undergo surgery. N Engl J Med. 2010;363:1597-607.

25. Thourani VH, Kodali S, Makkar RR, et al. Transcatheter aortic valve replacement versus surgical valve replacement in intermediate-risk patients: a propensity score analysis. Lancet. 2016;387:2218-25.

26. Reardon MJ, Van Mieghem NM, Popma JJ, et al. Surgical or Transcatheter Aortic-Valve Replacement in Intermediate-Risk Patients. N Engl J Med. 2017;376:1321-31.

27. Shreenivas SS, Lilly SM, Szeto WY, et al. Cardiopulmonary bypass and intra-aortic balloon pump use is associated with higher short and long term mortality after transcatheter aortic valve replacement: a PARTNER trial substudy. Catheter Cardiovasc Interv. 2015;86:316-22.

28. Drews T, Pasic M, Buz S, et al. Elective femoro-femoral cardiopulmonary bypass during transcatheter aortic valve implantation: a useful tool. J Thorac Cardiovasc Surg. 2013;145:757-63.

29. Meani P, Gelsomino S, Natour E, et al. Modalities and Effects of Left Ventricle Unloading on Extracorporeal Life support: a Review of the Current Literature. Eur J Heart Fail. 2017;19 Suppl 2:84-91. 
30. Sauer CM, Yuh DD, Bonde P. Extracorporeal membrane oxygenation use has increased by $433 \%$ in adults in the United States from 2006 to 2011. ASAIO J. 2015;61:31-6.

31. Raffa GM, Gelsomino S, Sluijpers N, et al. In-hospital outcome of post-cardiotomy extracorporeal life support in adult patients: the 2007-2017 Maastricht experience. Crit Care Resusc 2017;19(Suppl 1):53-61.

32. Stretch R, Sauer CM, Yuh DD, Bonde P. National trends in the utilization of short-term mechanical circulatory support: incidence, outcomes, and cost analysis. J Am Coll Cardiol. 2014;64:1407-15.

33. McCarthy FH, McDermott KM, Kini V, et al. Trends in U.S. Extracorporeal Membrane Oxygenation Use and Outcomes: 2002-2012. Semin Thorac Cardiovasc Surg. 2015;27:81-8.

34. Ibanez B, James S, Agewall S, et al. 2017 ESC Guidelines for the management of acute myocardial infarction in patients presenting with ST-segment elevation: The Task Force for the management of acute myocardial infarction in patients presenting with ST-segment elevation of the European Society of Cardiology (ESC). Eur Heart J. 2018;39:119-177.

35. Barbone A, Malvindi PG, Sorabella RA, et al. 6 months of "temporary" support by Levitronix left ventricular assist device. Artif Organs. 2012;36:639-42.

36. Tam DY, Jones PM, Kiaii B, et al. Salvaging catastrophe in transcatheter aortic valve implantation: rehearsal, preassigned roles, and emergency preparedness. Can J Anaesth. 2015;62:918-26.

37. Seiffert M, Conradi L, Baldus S, et al. Severe intraprocedural complications after transcatheter aortic valve implantation: calling for a heart team approach. Eur J Cardiothorac Surg. 2013;44:47884.

38. Malvindi PG, Raffa GM, Basciu A, et al. Bicuspidy does not affect reoperation risk following aortic valve reimplantation. Interact Cardiovasc Thorac Surg. 2012;14:717-20.

39. Perrotta S, Lentini S, Rinaldi M, et al. Treatment of ascending aorta disease with Bentall-De Bono operation using a mini-invasive approach. J Cardiovasc Med (Hagerstown). 2008;9:1016-22. 
40. Malvindi PG, Cappai A, Raffa GM, et al. Analysis of postsurgical aortic false aneurysm in 27 patients. Tex Heart Inst J. 2013;40:274-80.

41. Raffa GM, Kowalewski M, Brodie D, et al. Meta-Analysis of Peripheral or Central ECMO in Postcardiotomy and Non-Postcardiotomy Shock. Ann Thorac Surg. 2018 Jun 27. doi: 10.1016/j.athoracsur.2018.05.063.

42. Lester L, Brady MB, Brown CH 4th. Sedation Versus General Anesthesia for TAVR: Where Do We Go From Here? J Cardiothorac Vasc Anesth. 2017;31:2055-2057.

43. Konigstein M, Ben-Shoshan J, Zahler D, et al. Outcome of patients undergoing TAVR with and without the attendance of an anesthesiologist. Int J Cardiol. 2017;241:124-127. 


\title{
CHAPTER 6
}

Structured Review of Post-Cardiotomy Extracorporeal Membrane Oxygenation:

\author{
Part 1 - Adults Patients
}

Roberto Lorusso*, Giuseppe Maria Raffa*, Khalid Alenizy, Niels Sluijpers, Maged Makhoul, Daniel Brodie, Mike McMullan, I-Wen Wang, Paolo Meani, Graeme MacLaren, Mariusz Kowalewski, Heidi Dalton, Ryan Barbaro, Xiaotong Hou, Nicholas Cavarocchi, Yih-Sharng Chen, Ravi Thiagarajan, Peta Alexander, Bahaaldin Alsoufi, Christian Bermudez, Ashish S Shah, Jonathan Haft, David A D’Alessandro, Udo Boeken, Glenn Whitman

*Equally contributor

J Heart Lung Transplant. 2019 Nov;38(11):1125-1143 


\section{Abstract}

Cardiogenic shock, cardiac arrest, acute respiratory failure, or a combination of such events, are all potential complications after cardiac surgery which lead to high mortality. Use of extracorporeal temporary cardiocirculatory and respiratory support for progressive clinical deterioration can facilitate bridging the patient to recovery or to more durable support. Over the last decade, extracorporeal membrane oxygenation (ECMO) has emerged as the preferred temporary artificial support system in such circumstances. Many factors have contributed to widespread ECMO use, including the relative ease of implantation, effectiveness, versatility, low cost relative to alternative devices, and potential for full, not just partial circulatory support. While there have been numerous publications detailing the short and midterm outcomes of ECMO support, specific reports about post-cardiotomy ECMO (PC-ECMO), are limited, single-center experiences. Etiology of cardiorespiratory failure leading to ECMO implantation, associated ECMO complications, and overall patient outcomes may be unique to the PC-ECMO population. Despite the rise in PC-ECMO use over the past decade, short term survival has not improved.

This report, therefore, aims to present a comprehensive overview of the literature with respect to the prevalence of ECMO use, patient characteristics, ECMO management, and in-hospital and early post-discharge patient outcomes for those treated for post-cardiotomy heart, lung, or heart-lung failure. 


\section{Introduction}

Cardiogenic shock following cardiac surgery is a life threatening condition characterized by severe myocardial contractile impairment and reduced organ perfusion. In the current era, patient mortality approaches $40 \%{ }^{1}$ Acute mechanical support therapies are increasingly utilized in patients with progressive organ dysfunction despite optimized management, including mechanical ventilation, blood products and pharmacotherapy. ${ }^{1}$ Extracorporeal membrane oxygenation (ECMO) is increasingly used as first line mechanical circulatory support in patients refractory to conventional treatment. ECMO facilitates respiratory gas exchange and provides cardiac output to end-organs, supporting organ recovery, identification of residual lesions and allowing time for "bridge-to-decision" to more durable modes of support. ${ }^{1-6}$ ECMO is immediately available, easy to apply and reliably supports heart and lung functions - these features have made it an attractive option for circulatory support in the post-cardiotomy (PC) setting. ${ }^{1-6}$ In contrast, implantable or para-corporeal ventricular assist devices (VADs) are more complex, costly and do not facilitate respiratory gas exchange. These features have hindered widespread adoption in the immediate PC setting. ${ }^{7-12}$

PC-ECMO is the most frequent indication for ECMO in the United States. ${ }^{4}$ The exponential increase in ECMO use has not been accompanied by improved early survival, with some reports of increasing mortality in the last decade. ${ }^{6,13}$ Published reports of PC-ECMO are mainly single-center experiences ${ }^{14-37}$, frequently combining adult and pediatric patients in the same series, or including PC-ECMO in reports of institutional ECMO experience. $^{38-55}$ As a result, interpretation of PC-ECMO indications, complications and outcomes can be challenging. PC-ECMO is increasing, and uniquely complicated, making an analysis of PC-ECMO prevalence, patient selection, in-hospital management, and short- and long-term outcomes relevant to the multi-disciplinary team managing these patients.

PC-ECMO is being increasingly used in both adults and pediatric populations. There are, however, distinct differences between PC-ECMO in these groups and this review will focus on adult cardiac surgery patients only. The authors aim to provide a detailed and comprehensive evaluation of the adult PC-ECMO and present outcomes for veno-arterial (VA) and veno-venous (VV) ECMO. Trends in PC-ECMO use, educational needs, and future perspectives will be addressed to highlight potential applications, required training, and implications for future research. An accompanying review will address PC-ECMO in the pediatric population. 


\section{Characteristics of PC-ECMO}

\section{Trends in use}

The incidence of ECMO implementation in patients after open-heart surgery has been reported between $0.4 \%$ to $3.7 \%$ (Table 1). PC-ECMO has increased considerably over the past 2 decades. $^{2}$ Indeed, from 2007 to 2011 , non-percutaneous ECMO cannulation increased 2-fold, while the use of percutaneous ECMO increased by more than 15 -fold. ${ }^{2}$ Maxwell and colleagues, evaluating more than 9000 ECMO patients from the Nationwide Inpatient Sample database in the United States from 1998 to 2009, identified 4493 patients with approximately 50\%, cannulated for cardiogenic shock in the post-operative period.. ${ }^{5}$ Between 2002 and 2011 in the same database, McCarthy and colleagues found that PC-ECMO was the most common indication for ECMO in each year of their study. ${ }^{3}$ Recently, data from the Extracorporeal Life Support Organization (ELSO) Registry also confirmed a substantial increase in PC-ECMO use over the last 10 years. ${ }^{6}$ ECMO use in adult populations has also substantially increased in this time, such that the proportion of adults managed on ECMO for PC indication has decremented..$^{5,3}$

\section{Patient characteristics}

The nature of the PC-ECMO indication is that patients invariably require urgent or emergent ECMO support, compared to other indications. Baseline characteristics reveal PC-ECMO patients are older, with higher incidence of renal insufficiency, prior myocardial infarction, left main coronary artery disease, and left ventricular (LV) dysfunction. They are also more likely to have a prolonged history of coronary artery disease and prior open-heart surgery. ${ }^{17}$ Age does not appear to be an absolute contraindication as elderly patients are routinely considered for PC-ECMO. Indeed, most PC-ECMO series include some patients older than 80 years of age (Table 1). ${ }^{56,57}$

As expected, PC-ECMO is most frequently associated with coronary artery bypass grafting, valve surgery, and combination of valve/coronary surgery (Table 1). In several series, diagnoses previously considered relative contraindications to ECMO, such as repair of an acute aortic dissection, have been successfully supported (Table 1). In patients post-heart transplantation, primary graft failure was associated with early post-transplant mortality. The use of ECMO post-heart transplant has been reported to be as high as $10-15 \%{ }^{58-60}$, possibly associated with the increased use of marginal donors. Notably, in a series of 124 heart transplants, Listijono and colleagues showed that ECMO was used for $17(14 \%)$ patients with $82 \%$ surviving to discharge. ${ }^{62}$ In 
patients who received a marginal donor heart, defined by a pre-transplant LV ejection fraction $<45 \%, 8$ out of 9 patients were managed with ECMO, and of these $88 \%$ survived to hospital discharge with normal graft LV function. ${ }^{62}$ Another indication for ECMO is in patients who develop right ventricular (RV) failure post-LVAD implantation. ${ }^{63,64}$ PC-ECMO may be utilized to support the RV, or to bridge to right ventricular assist device (RVAD). ${ }^{64,65}$

\section{Indications}

The most common indication for PC-ECMO implementation is intraoperative failure to wean from cardiopulmonary bypass $(\mathrm{CPB})$ due to univentricular, biventricular, or respiratory failure. PC-ECMO may also be implemented for delayed refractory cardiogenic shock, postoperative cardiac arrest in the intensive care unit (ICU), respiratory failure, or intractable post-operative ventricular arrhythmias. ${ }^{14-35}$ Although not all series delineate the specific diagnosis and indication for PC-ECMO, it is evident that the use of ECMO for PC cardiac arrest has been more frequently considered during the last 10 years. ${ }^{66}$ This is reflected in the 2017 Society of Thoracic Surgeons Expert Consensus for the Resuscitation of Patients Who Arrest After Cardiac Surgery wherein it is recommended that failure to achieve spontaneous circulation is an indication for open cardiac massage and institution of either central or peripheral ECMO at the bedside. ${ }^{67}$ Available information with regards to PC-ECMO applied for perioperative cardiac arrest and related outcomes are presented in the Supplemental Materials (Table 1 Supplemental Data).

\section{Implementation, Management, and Complications of PC-ECMO}

\section{Cannulation}

Location of ECMO cannulation is influenced by the timing and indication, urgency of deployment, cardiocirculatory vs. respiratory support required, and institutional factors including staff familiarity and availability of ECMO circuits. PC-ECMO is most often utilized for failure to wean from $\mathrm{CPB}$, so operating room cannulation occurs most frequently, followed by the ICU, and rarely on the ward (Table 2). While PC-ECMO can be initiated any time in the postoperative course, the majority of PC-ECMO placements occur within 24 to 48 hours of procedure (Table 2).

Implementation of intra-operative ECMO generally follows a period of escalating vasoactive support and may be delayed by the placement of an intra-aortic balloon pump (IABP). There is limited data to inform optimal 
timing of ECMO cannulation, either in the fail to wean from CPB population, or in those cannulated later in the ICU. Institutional protocols differ with respect to escalation of vasoactive therapies, optimal preload and acceptable duration of myocardial and systemic exposure to high dose inotropes. In the ICU, timing of PCECMO cannulation may be even more variable, as indications are not well defined, which may contribute to delay in decision making resulting in ECMO deployment. Furthermore, there is institutional variability in logistics for ECMO circuit preparation and implementation. ${ }^{30}$ ECMO deployed in the setting of cardiopulmonary resuscitation (ECPR) without return of circulation may present a more straightforward decision, but with more complicated logistics and event management. ${ }^{67}$ Although post-ECMO survival in similar for those cannulated to ECMO in the operation room and the ICU, longer ICU duration prior ECMO cannulation is associated with poorer survival. ${ }^{22}$

Despite the presence of either a median sternotomy or thoracotomy which allows central access, peripheral cannulation (Figure 1) is more common than central cannulation for PC-ECMO (Table 2). Interestingly, a peripheral approach was the only access employed in 3 series $^{21,26,29}$, whereas a central approach was exclusively used for access in only one center's experience (Table 2$) \cdot{ }^{28}$ Peripheral cannulation may be preferred in order to reduce infection risk, avoid re-sternotomy, and continue uninterrupted chest compressions during ECMO cannulation.

In studies of PC-ECMO describing peripheral cannulation, open cannula placement was chosen over a percutaneous approach in the majority of cases. ${ }^{18,26,28}$ There is evidence that this approach is associated with fewer complications than percutaneous cannulation..$^{27,29,68}$ A "pseudo-percutaneous approach" exposes the femoral vessels, as with an open approach, but tunnels the cannulas through a more inferior thigh incision before inserting them into the vessel, allowing closure of the femoral incision. ${ }^{69-71}$ This pseudo-percutaneous approach reduces the risk of bleeding and infection post-ECMO implantation and facilitates more straightforward device removal. Although an open surgical closure is still needed, the surgeon has better control of the vascular entry site and can perform embolectomy more easily in patients with distal or proximal thromboses. Nevertheless, Rastan and colleagues showed that femoral venous drainage was associated with worse prognosis suggesting that suboptimal right-sided decompression had a negative impact on ECMO flow and management. ${ }^{22}$ 
Central PC-ECMO cannulation is associated with higher rates of bleeding and acute renal failure requiring continuous VV hemofiltration when compared with peripheral cannulation, but a recent meta-analysis found no difference in overall survival when compared to peripheral cannulation. ${ }^{44}$ Technically, emergent central cannulation is associated with risk of air entrainment and embolization. The benefit of central cannulation lies in improved cardiac decompression and anterograde flow from the proximal aorta, preventing the potential 'Harlequin syndrome'. Alternative cannulation strategies, e.g. arterial inflow via the subclavian artery with either peripheral ${ }^{20,21,33,69-71}$ or central ${ }^{68}$ cannulation for venous return have also been employed (Figure 2). Central cannulation generally requires an open sternum to allow unimpeded exit of atrial and aortic cannulas, and to prevent mechanical cardiac compression which may inhibit venous return. ${ }^{22,27}$ Innovative strategies to manage the atrial and aortic cannulas may result sternal closure with tunneled cannula exit in the subxyphoid region (Figure 3). These strategies may facilitate patient extubation, mobilization, and decreased risk of infection. Such a configuration, however, may impede adequate cardiac filling during weaning because of RV compression exerted by cannula course in the mediastinum. In patients who are expected to have an expeditious recovery after short-term support, suprasternal exit sites, by mobilizing the sternocleidomastoid muscle insertions and extending the upper sternal incision with a $\mathrm{Y}$ towards the head, may be contemplated (Figure 4). This approach allows the aortic and atrial cannulas to lay vertically and exit cranially, preventing cardiac compression and tamponade during weaning trials. The use of a right anterior mini-thoracotomy for cannulation of the ascending aorta, right atrium and pulmonary vein (for venting) (Figure 5), has also been reported and may be also useful in avoiding any cardiac compression. ${ }^{71}$

\section{Left ventricular decompression}

VA-ECMO, particularly with central cannulation, usually results in effective right-sided cardiac drainage but may not be effective in decompressing the left side of the heart due to incomplete capture of systemic and bronchopulmonary venous flow. LV distension occurs due to insufficient ejection, either from impaired LV contractility or high afterload, and inevitably raises wall tension. Increased LV and resulting left atrial pressures results in increased myocardial oxygen consumption and acute pulmonary edema. Furthermore, inadequate LV ejection results in blood stasis in the left cardiac chambers and may lead to clot formation with devastating consequences. In these circumstances, the addition of left sided catheter drainage may be warranted to fully decompress the left side of the heart. ${ }^{72}$ In the presence of LV distension and left intra-ventricular or 
intra-atrial stasis, LV decompression can be accomplished by surgically placed catheter in the left atrium or ventricle, or use of a trans-aortic valve axial pump device (if no aortic mechanical prosthesis is in place, Table 2 Supplemental Data). In a recent meta-analysis of 17 observational studies including almost 4.000 VA-ECMO patients, $42 \%$ of the cohort utilized an LV unloading device - IABP in $91.7 \%$ of cases, a percutaneous device in $5.5 \%$, and trans-septal cannulation in $2.8 \%$. There was significantly lower mortality in the combined device group (53\%) compared to ECMO without LV unloading $(65 \%) .{ }^{73}$ Patients with a mechanical prosthesis are at particularly high risk of valve thrombosis and dysfunction with poor LV ejection, increasing risk of intracavitary clot formation. For these at-risk patients, a higher level of blood anticoagulation may be advisable when unloading the LV.

Pulmonary artery cannulation represents an alternative to direct LV unloading, but is not available to all patients. The use of a trans-aortic valve axial pump device has been reported to be beneficial in ECMO patients. ${ }^{74,75}$ Patel and associates showed improved in-hospital survival (43\% vs. $22 \%$ ) with the use of a transaortic device in combination with VA ECMO ${ }^{76}$ Significantly higher than anticipated risk-adjusted survival was reported in 106 patients undergoing combined VA ECMO with the use of a trans-aortic valve device (Impella ${ }^{\circledR}$, Abiomed) ${ }^{77}$ Despite these data, there are no reports of trans-aortic valve axial pump device utilization in the PC-ECMO setting. The benefits include LV unloading with theoretically improved LV recovery, while complications such as hemolysis with renal failure, and an increase in surgical complexity (Table 2 Supplemental Data) warrant further investigation to evaluate the impact of this configuration. ${ }^{72}$

Concomitant use of IABP in patients on ECMO support for cardiogenic shock remains controversial. IABP remains the first line of support used in the operating room for cardiovascular failure. ${ }^{2,8}$ Combined with VAECMO, the IABP is used to improve coronary perfusion, support LV ejection and blood flow pulsatility by decreasing afterload, decreasing LV wall tension and thereby decreases blood stasis in the left heart. ${ }^{20,21,72,78,79}$ In multiple PC-ECMO series that we have reviewed, an IABP was used concomitantly in $12 \%$ to $100 \%$ of patients (Table 2). Although this institutional variability may represent patient heterogeneity, more likely these differences in utilization reflect a lack of consensus regarding relative benefit of IABP in ECMO supported patients. ${ }^{32,33,78,79}$ Consistent with this is the fact that in some PC-ECMO series, IABP use was associated with improved survival ${ }^{17,22}$, while in others, no survival differences were found. . $^{19,23,24,26,27,40,47}$ Reassuringly, although the IABP competes for femoral arterial access, conceivably problematic in peripheral ECMO, there 
does not appear to be an increase in the risk of limb ischemia, as those authors uniformly using an IABP in patients on PC-ECMO showed very low rates of limb-related complications. ${ }^{14,21,25}$ Despite the physiologically justification and early reports of results, the clinical benefits of LV decompression remain unproven.

\section{Management of patients on PC-ECMO}

The management of patients supported by PC-ECMO differs substantially from the management of patients on ECMO support for other indications. Firstly, adult cardiac surgery patients often have significant comorbidities which may impact the outcomes of ECMO. Secondly, ECMO may be used as a bridge-torecovery and also as bridge-to-advanced mechanical circulatory support. PC- ECMO is only rarely used as a bridge-to-decision, as the typical cardiac surgery patient has been well characterized clinically during the preoperative work up, and goals of therapy are clear. Optimal ECMO flow remains unclear, as some argue that allowing LV ejection might be superior to full support, taking into account the magnitude of heart damage while assessing the need for adequate peripheral perfusion. ${ }^{80}$ As previously described, facilitating an injured LV to eject adds additional myocardial work and energy consumption to an already compromised heart. Finally, bleeding and coagulopathy are major issues in this setting.

In some patients, the need for perioperative ECMO may be predictable, based on severe clinical or cardiocirculatory-pulmonary conditions, and assessed preoperatively or intraoperatively, thereby pre-empting cardiac failure post-CPB. In these patients, prophylactic ECMO initiation may be of benefit, despite associated risk of complications, as it allows a smoother perioperative course with reduced use of vasopressors and avoidance of severe hemodynamic compromise. ${ }^{81}$

The use of vasoactive medications remains another controversial issue in patients supported with ECMO, particularly PC-ECMO. As stated above, support of cardiac contractility may improve LV unloading, prevent intracardiac hemostasis, as well as to support ECMO weaning ${ }^{80}$, at the expense of increasing myocardial work which may impede recovery. Recently, it was shown that pre-treatment with levosimendan (Symdax $\left.{ }^{\circledR}, A b b o t\right)$, a calcium-sensitizing inodilator seems to facilitate weaning from VA ECMO, reducing the use of high-dose inotropes. ${ }^{82}$ Weaning rates were $83.3 \%$ with vs. $27.3 \%$ without levosimendan infusion. ${ }^{82}$ However, these data are preliminary and require confirmation. ${ }^{82,83}$ The degree of contractility necessary to protect the unvented heart in the setting of ECMO is unclear, and thus, there is no definable goal for inotropic support. This aspect of ECMO management requires investigation. 


\section{Duration of PC-ECMO support}

Duration of ECMO support varies between reports, but patients are rarely supported longer than 15 days for PC indication (Table 2). Several investigators advocate short (48-72 hours) support times and, if insufficient recovery is observed, implementing more advanced mechanical support. ${ }^{16,21,26}$ This reflects two important aspects of PC-ECMO in adult patients: 1) if recovery occurs, it is usually observed early after surgery ${ }^{84}$, and 2) severe and irreversible complications are frequent and typically occur shortly after ECMO initiation, resulting in high mortality. ${ }^{6,22}$

Longer duration of ECMO support may be required and effective in specific patients, such as patients in need of circulatory support after heart transplantation, to allow myocardial recovery or resolution of refractory pulmonary hypertension. ${ }^{16}$ However, for those patients post-cardiac surgery, lack of early recovery should precipitate transition to more durable forms of support, e.g. LVAD or heart transplant listing. Unfortunately, these therapies are not universally available. This speaks to the need for a relationship between all cardiac programs and a sophisticated heart failure centre (with VAD and heart transplant expertise) to facilitate patient transfers when indicated.

\section{Complications associated with PC-ECMO}

Complications in ECMO patients are common and frequently determine a patient's final outcome. ${ }^{85}$ Table 3 shows the most common adverse events associated with PC-ECMO. In published reports limited information is often provided regarding the specific complications encountered, rather they are categorized in broad terms (e.g. clot, hemorrhage, stroke), hampering any in-depth analysis. However, bleeding is the most frequent complication encountered, occurring in up to $90 \%$ of patients in some series. ${ }^{14,20}$ PC-ECMO patients are at high risk for hemorrhagic events as typical postoperative surgery-related bleeding is magnified by the early need for anticoagulation required for the ECMO circuit. Furthermore, coagulopathy and bleeding encountered may be exacerbated by a recently highlighted, heparin-like effect induced by ECMO, even when direct thrombin inhibitors are employed as alternatives to heparin. Ranucci and colleagues detected a heparin-like effect in 23 of 41 patients (56\%), most likely due to a release of heparinoids from the glycocalyx or mast cells, as the result of the systemic inflammatory response to the ECMO circuit or sepsis. ${ }^{39}$ To avoid exacerbating the coagulopathy that is already present post bypass, many investigators advocate avoiding heparin infusion for 
the first 12-48 hours, provided that high flows are maintained to prevent clot formation. ${ }^{18,21}$ Heparin can be withheld for even longer periods in patients with continued postoperative bleeding. ${ }^{16,18,21,22,24,30,47}$

There are additional adverse events, similar to those seen in other ECMO settings, but exacerbated as a result of the preceding CPB run and cardiac surgery. Central nervous system complications have recently been shown to occur in $15 \%$ of adult patients supported with VA ECMO and $7 \%$ of adult patients supported with VV ECMO.$^{86,87}$ In PC-ECMO, these rates may be much higher, occurring in up to $30 \%$ of patients. ${ }^{14,16,17,21}$ The caregiver must remain cognizant of the high rate of neuro-complications. A high level of suspicion, appropriate neuro-monitoring, and prompt execution of ECMO management modifications, including a low threshold for neuroimaging, all play an important role in the care of the PC-ECMO patient ${ }^{88}$ Interestingly, it appears that elderly patients are not at higher risk for neurologic adverse events when compared with younger patients in PC-ECMO setting. ${ }^{30}$ A study of the ELSO registry showed equal rates of cerebral injury in patients older than 70 years of age who underwent ECMO for cardiogenic shock. ${ }^{85}$

In contrast to the higher rates of neurological adverse events in PC-ECMO, renal failure and limb ischemia occur at rates similar to other indications for ECMO. Several investigators have consistently reported reduced limb ischemia with routine use of ipsilateral distal perfusion. ${ }^{22}$ Delay in cannula placement, triggered by signs of ischemia, may lead to a reperfusion injury with a poor outcome ${ }^{28}$ (Table 3 Supplemental Data). Although distal femoral artery cannulation placed directly into the vessel is easy and effective, femoral artery access can also be achieved by placing a femoral artery chimney graft (a Dacron ${ }^{\circledR}$ or Hemashield ${ }^{\circledR}$ prosthetic graft) of 6-8 $\mathrm{mm}$ diameter, anastomosed end-to-side to the femoral artery, thus maintaining antegrade and retrograde arterial flow to the ipsilateral lower limb. ${ }^{21}$ In PC-ECMO, continuous near-infrared spectroscopy monitoring of the adequacy of limb perfusion is no different from the management applied in other ECMO settings with peripheral arterial access. ${ }^{71}$

Reassuringly, ECMO circuit failure occurs at a very low rate (Table 3). Although circuit failure may be underreported, the general consensus is that current ECMO technology is safe with a low incidence of catastrophic complications due to component failure or dysfunction. Most of the time, these events are foreseeable and involve the pump-head or oxygenator. Even so, further improvements in circuit components, including greater biocompatibility, are expected in the coming years.

\section{Post-cardiotomy ECMO Outcomes}




\section{Weaning from PC-ECMO and survival to hospital discharge}

As expected, successful weaning from PC-ECMO varies greatly within published series, ranging from $31 \%$ to $76 \%$, with almost half of the published experiences showing a weaning rate at or slightly above 50\% (Table 4). Survival to hospital discharge rates are far less, ranging from $16 \%$ to $52 \%$, with fewer than $30 \%$ of centers reporting survival-to-discharge above $40 \%$ (Table 4). Of note, even in the face of considerably improved technology and increased experience in managing ECMO care, survival has not improved in the last 20 years. ${ }^{24}$ In fact, based on the most recent report from the ELSO Registry, there has been a gradual decline in survival after PC-ECMO, as low as $15 \%$ survival in a recent analysis. ${ }^{6}$ This may be due, at least in part, to more widespread application of this technology to higher risk patients, but it remains a sobering observation. The presence of cardiac arrest as indication for PC-ECMO is understandably a negative predictor of weaning or survival to-discharge in all series examined (Table 1 Supplemental File); however, this must be viewed as preliminary data, as only two papers specifically address ECPR in this setting. ${ }^{32,33}$ Survival in patients with cardiac arrest ranged from $0 \%$ to $48 \%$, but factors such as quality of cardiopulmonary resuscitation (CPR), e.g. open vs closed chest, the location of arrest, e.g. in the operating room or in the ICU, and time to ECMO institution, all likely of paramount importance. An intraoperative onset arrest might have a better prognosis due to more rapid ECMO cannulation and limited hypoperfusion-based organ injury, but this hypothesis has not been explored.

Multi-organ system failure, despite recovery from myocardial failure is an important contributor to mortality, but granular data to inform this observation are lacking. ${ }^{20,21}$ In fact, the actual cause of death may be interpreted in a misleading fashion in ECMO patients, as reported by Rastan and associates who showed that in almost $30 \%$ of autopsies an unexpected cause of death was found, and in $80 \%$ of the patients, an unrecognized concomitant illness was present. ${ }^{90}$ Overall, that study showed mortality due to a cardiac etiology in $>60 \%$ of patients, multi-organ system failure in $10 \%$, and neurologic complications in $5 \% .^{90}$

The survival of patients who received PC-ECMO for post-heart transplant graft dysfunction is approximately $45 \%$, better than typically seen in PC-ECMO, as post graft dysfunction is frequently reversible. ${ }^{16,18,29,91}$ Takeda and colleagues recently compared patient outcomes after primary graft failure with PC-ECMO support versus VAD support, and showed that PC-ECMO had significantly better outcomes including bleeding after postimplant re-thoracotomy ( $30 \%$ vs. $70 \%$, p $<0.01)$, renal replacement therapy $(11 \%$ vs. $53 \%$, p $<0.01)$, duration 
of support ( $5.2 \pm 3.9$ days vs. $14 \pm 17$ days, $\mathrm{p}=0.011)$, and weaning rates $(89 \%$ vs. $59 \%, \mathrm{p}=0.03)$, with a trend towards better survival-to-hospital discharge (41\% vs. $19 \%) .{ }^{92}$ The favorable impact of ECMO on patients post-heart transplant as compared to other settings, has been highlighted by other investigators, showing significantly better in-hospital and post-discharge survivals..$^{93,94}$

\section{Outcomes in elderly patients}

The effect of patient age on survival requires highlighting in the context of PC-ECMO as many eligible patients in this setting are elderly. In an analysis of 131 adult patients $(28 \% \geq 65$ years of age) with refractory cardiopulmonary failure supported by ECMO, Narotsky and colleagues observed $48 \%$ survival at one year. ${ }^{51}$ Age over 65 years was associated with $\sim$-fold higher risk of death. ${ }^{51}$ However, when the analysis was adjusted for confounders, age was not a statistically significant predictor for in-hospital death, though a trend for increased risk remained. ${ }^{51}$ Saito and colleagues analyzed 91 patients who required emergency ECMO for a variety of reasons including PC, and found that age was not a predictor of mortality, concluding that elderly patients can benefit from ECMO, although they require more time to recover. ${ }^{41}$ However, Elshakarwy et al found a linear relationship between mortality after PC-ECMO support and age, but there was no identified age -specific cut off after which ECMO was futile. ${ }^{23}$ Consistent with this, a recent large database evaluation demonstrated that older patients who require ECMO support for cardiac or respiratory indications have a significantly worse prognosis; nevertheless, the data does not appear to support older age as an absolute contraindication to PC-ECMO ${ }^{89}$ These findings create a conundrum for surgeons and patients' relatives when considering ECMO support for an elderly patient. Survival rates in elderly patients who undergo ECMO for cardiogenic shock may range between $25 \%$ and $30 \%{ }^{30,89}$ These survival rates may be influence by selection bias, but it is encouraging that well selected elderly patients managed on ECMO may do well. Additional investigation is needed to elucidate which older patients have the best survival after PC-ECMO. At this time limited information is available on mid- and long-term patient disability after PC-ECMO, and one must conclude that further experience is needed to best determine in whom it is contraindicated.

\section{Bridging to other therapies}

Transitioning to VAD support or heart transplantation listing from PC-ECMO appears to improve in-hospital survival, as shown by Smedira and coworkers. ${ }^{17}$ They found a $75 \%$ early survival in ECMO patients bridged to a VAD after a short ECMO run. ${ }^{17}$ Despite this apparent effectiveness, less than $20 \%$ of patients supported 
with PC-ECMO transition to other support, e.g. VAD or heart transplantation (range $0 \%$ to 20\%) (Table 4), totally dependent upon the suitability of the candidate for more durable therapies and the availability of huband-spoke networks allowing patient transfers to centers capable of these advanced heart failure options.

\section{Predictors of outcomes after PC-ECMO}

Prediction of ECMO weaning and survival after PC-ECMO has been addressed by many investigators. ${ }^{14-35}$ Pre- and intra-operative factors, post-ECMO events, and the patient's physiologic response to circulatory support, all play a critical role (Table 4). Among pre-ECMO factors, ECPR was a strong negative predictor of survival in several experiences. ${ }^{14,16,30}$ In 2 series, no post-cardiotomy patient who underwent ECPR and was elderly or who had prolonged CPR prior to establishment of ECMO support survived. ${ }^{16,30}$ However, other investigators have found that neither CPR nor the time spent in resuscitation had a significant effect on survival. ${ }^{18,32,33}$ Currently, the following factors negatively influence survival after PC-ECMO support: lactate concentration immediately prior to ECMO initiation as well as its highest level 12-48 hours post-ECMO initiation $^{19,22,26,29,30,35,37,43,84}$, renal and liver failure ${ }^{95-97}$, respiratory failure ${ }^{98,99}$, and duration of ECMO support. 16,18,26,27 Regarding pre-ECMO lactates, Fux and collaborators have recently shown that a value above 10 $\mathrm{mmol} / \mathrm{L}$ at implant is associated with a $90 \%$ in-hospital mortality, with no survival when lactate levels were greater than $15 \mathrm{mmol} / \mathrm{L} .{ }^{37}$ Surprisingly, neither ECMO cannulation location (intra-operative, ICU, or ward ${ }^{27}$ nor the duration of ECMO support has been shown to impact survival. ${ }^{19,22,24,30}$ Other predictors of success in weaning or survival after PC-ECMO are reported in Table 4.

\section{Long-term outcomes after PC-ECMO}

Despite the complex perioperative course of PC-ECMO patients, long-term survival of patients who survive to discharge appears favorable, with the vast majority of patients still alive at 1-year follow-up (Table 4). Saxena and colleagues showed that even in a high-risk cohort of patients over 70 years of age survival was $69 \%$ at 3 years, and $51 \%$ at 5 years, confirming good post-discharge prognosis of PC-ECMO patients, even the elderly. ${ }^{30}$

\section{Post-Cardiotomy Veno-Venous ECMO for Respiratory Dysfunction}

Respiratory insufficiency is a common complication after cardiac surgery and an independent predictor of inhospital mortality. ${ }^{98,99}$ Despite respiratory complications in $7-30 \%$ of patients, ${ }^{98,99}$ there is a paucity of 
published reports on ECMO therapy when this complication is severe, treated either with VA- or VV-ECMO. VV-ECMO has been increasingly employed as therapy for primary, refractory respiratory failure as a result of the outcomes seen in conventional ventilatory support vs ECMO for severe adult respiratory failure in the CESAR trial. ${ }^{100}$ There are very few reports, however, specifically addressing the use of VV-ECMO in PC patients. Nakamura and colleagues explored the outcomes of VV-ECMO in 11 PC patients, ranging in age from 35 to 83 years, after various cardiac surgery procedures. ${ }^{56}$ This series showed favorable overall outcomes (7 patients were discharged, 64\%), and age was the only predictor of a poor outcome. ${ }^{56}$ The mean age of the survivors was 54 years while non-survivors were mean 80 years old. ${ }^{56}$ Interestingly, Song and co-workers recently reported their experience in 13 PC patients supported with VV-ECMO for acute respiratory distress syndrome, and age did not predict survival (overall in-hospital survival 69\%). ${ }^{57}$ In this series, 7 patients received VV-ECMO within 24 hours of surgery and the remainder (6 patients) at a median of 8.5 days from surgery. ${ }^{57}$ They showed that the Respiratory ECMO Survival Prediction score (RESP) ${ }^{101}$ proved to be a good predictor of survival (100\% in class III, $50 \%$ in class IV, and $20 \%$ in Class V).${ }^{57}$ Noteworthy, in this experience, septic shock was responsible for all deaths during ECMO support. ${ }^{57}$ Further research is needed to understand the indications for VV-PC-ECMO and to systematically minimize pre and intraoperative risk factors for PC respiratory failure requiring VV-ECMO, e.g. smoking cessation, avoiding ventilation-induced lung injury, decreasing blood exposure, and minimizing CPB times.

\section{Controversial Issues and Future Perspectives on PC-ECMO}

ECMO technology has undergone remarkable progress in the last 20 years. More advanced, user-friendly, miniaturized technology has made the wider application of this temporary support possible, in many instances for conditions once viewed as contraindications. ${ }^{12,102}$ Obvious targets to improve effectiveness include biocompatible circuitry (e.g. the pump, oxygenator, and tubing design), more reliable anticoagulation, rational vasoactive/inotropic support, a better understanding of the most effective ways to achieve temporary cardiopulmonary support, more effective monitoring, and increasing provider skill and education. Given the technical aspects of ECMO implementation, the peculiarities of ECMO management, and the expense and ethical implications of ECMO use, universal provider education is a crucial target if outcomes are to improve. ${ }^{103,104}$ Available ECMO courses, case-presentations and simulation training with a multidisciplinary 
target audience may mitigate the lack of high volume ECMO use at most centers and improve standardization and evidence-based care for all. ${ }^{104,105}$

As we witness an exponential increase in ECMO use, we must question in whom is it truly beneficial, determining in whom it provides time to recover as opposed to simply prolonging death. ${ }^{104}$ ECMO enthusiasts are particularly vulnerable to criticism. ${ }^{6}$ Because ECMO is easy to institute, it is being used in patients with increasing disease severity, more complex procedures with acute, post-bypass myocardial dysfunction, and in patients who heretofore were viewed as unsalvageable..$^{30,39,40,50,87,102}$ Reoperations, advanced age, surgical urgency, and poor cardiac reserve are being seen more commonly and contribute to cardiopulmonary insufficiency post-CPB, with subsequent pressure on the caregiving team to institute PC-ECMO. It is also possible that PC-ECMO is being over-utilized to avoid intraoperative deaths, a rarity these days, moving the inevitable from the operating room to the ICU. ${ }^{11,103}$ However, PC-ECMO is still in its infancy and suffers from a lack of sufficient evidence to inform optimal use, e.g. patient selection, ECMO configuration, prevention or management of complications, and decision making for recovery or exit strategies of more durable therapies. To this end, the authors propose concentrating resources to address the following areas to improve PC-ECMO effectiveness: 1) the underlying pathophysiology of PC cardiopulmonary failure and the impact of ECMO on those processes, 2) the development of a standardized, evidence-based, structured approach to monitoring ECMO delivery and patient outcomes, 3) the identification of both patient- and population-specific predictors of outcomes, 4) the education and training of ECMO caregivers, and, finally, 5) the ethical and economic implications of ECMO utilization. For sure, the dilemmas associated with ECMO are all part of a complex scenario, but, to a large degree, providers must accept responsibility for shepherding use, despite the lack of data to inform them. ${ }^{6,104}$ Pre-operative discussion with high-risk patients and their families about the use of PC-ECMO should be encouraged to minimize ethical issues that may arise postoperatively. The futility of ECMO in complex patients with limited or no indication for more advanced treatment should be considered in a timely manner.

\section{Limitations of the Review}

This review has some limitations as it encompasses different patient conditions and different ECMO approaches in its attempt to be comprehensive. Nevertheless, underlying cardiopulmonary insufficiency and a 
lack of response to conservative, conventional treatments after cardiac surgery are common to all the included studies. The scenarios for PC-ECMO include conditions ranging from failure to wean from CPB, to cardiogenic shock hours to days after cardiac surgery, to cardiac arrest in the ICU or on the ward. PC-ECMO in heart transplant recipients is usually included in PC-ECMO series, as we have done, but this specific patient population tends to have better outcomes as compared with non-transplant patients. ${ }^{47,53}$ In addition, the studies reviewed include a wide range of resuscitation times prior to ECMO initiation, and in some circumstances, include patients who need both cardiac and respiratory support postoperatively. This highlights the extreme variability in the postcardiotomy patients who received PC- ECMO. Finally, institutional variability in ECMO management is high, and may impact results of studies and how the associated data has been interpreted. ${ }^{47,53}$

\section{Conclusions}

PC-ECMO represents the most frequent indication for temporary mechanical circulatory support with increasing use expected in the future. Considerable variability regarding surgical access and cannula placement still exists, apparently without major differences in outcomes regardless of the technique used. Although PCECMO can be life-saving and is only employed when there are few alternatives, mortality and morbidity remain high, reflecting underlying disease severity and an imperfect solution. When PC-ECMO is determined to be the best option for patient care, its deployment should be rapid as delay, with a resultant longer duration of circulatory hypoperfusion and hypoxia, is related to increasingly poor outcomes. Survivors of PC-ECMO have favorable early outcomes. Long-term survival has not been as well-studied, although some small series show good 1- and 3-year survival. ECMO-specific educational training programs focusing on patient selection, cannulation techniques, ECMO management, and ethical considerations, along with industry-driven refinements to circuit components, will almost certainly improve the effectiveness of this powerful technology. 


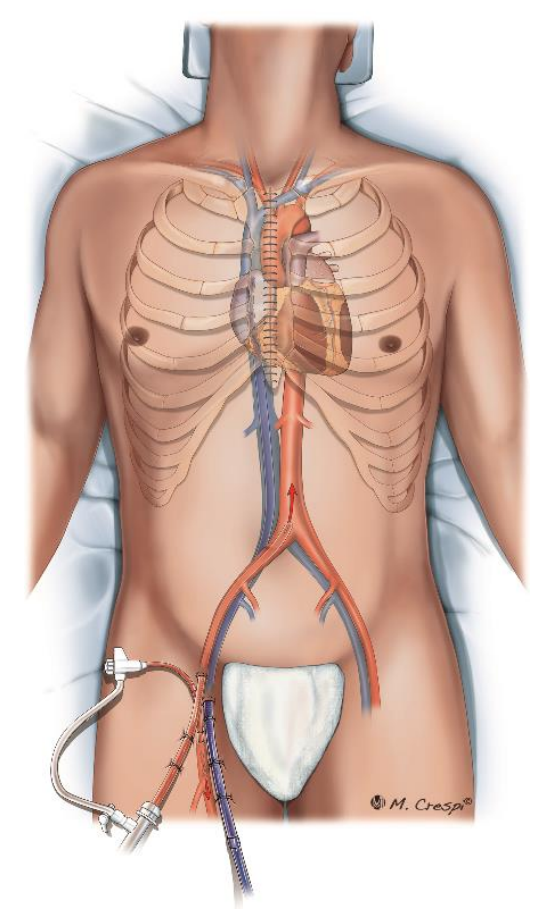

Figure 1. Post-cardiotomy ECMO approaches for cannulation: peripheral cannulation with femoral artery and vein access, associated with distal limb arterial perfusion.

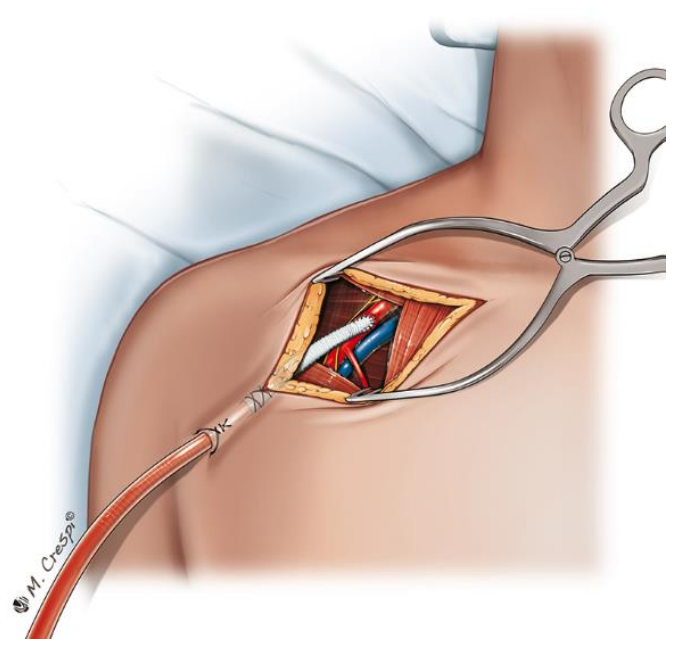

Figure 2. Post-cardiotomy ECMO approaches for cannulation: cannulation of the right axillary artery for reperfusion. Femoral vein or right atrial access can be used for venous drainage. 


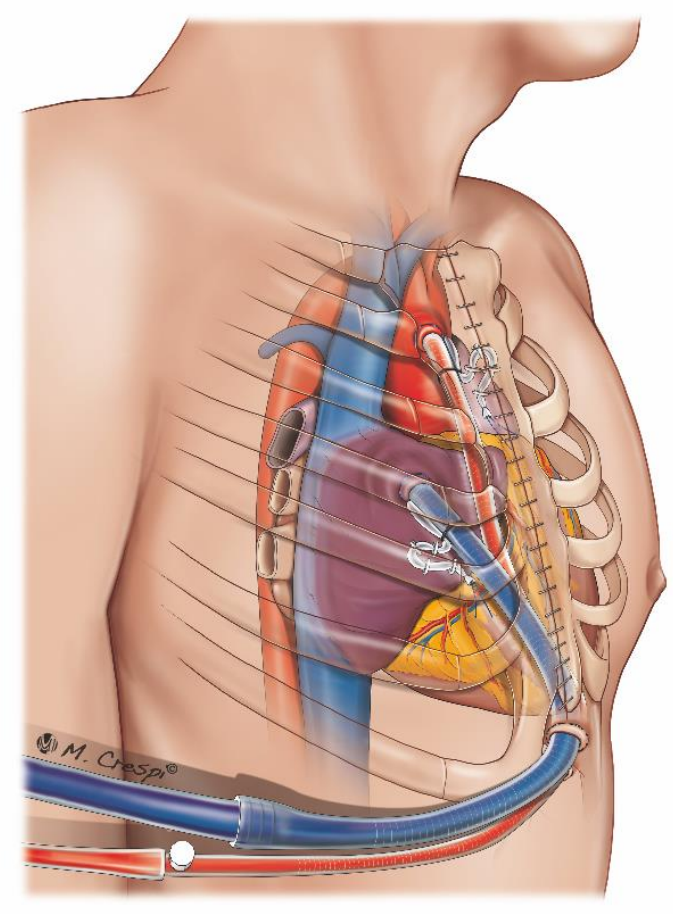

Figure 3. Post-cardiotomy ECMO approaches for cannulation: central cannulation (right atrium and ascending aorta cannulation) with a subxyphoid exit port for the cannulas.

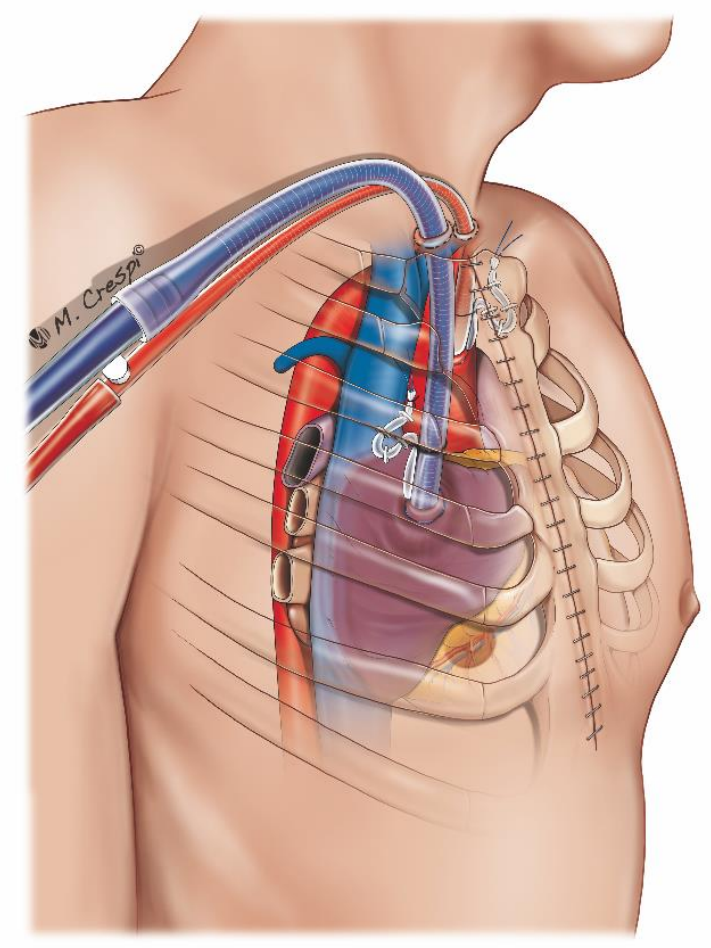

Figure 4. Post-cardiotomy ECMO approaches for cannulation: central cannulation (right atrium and ascending aorta cannulation) with jugular exit port for the cannulas. 


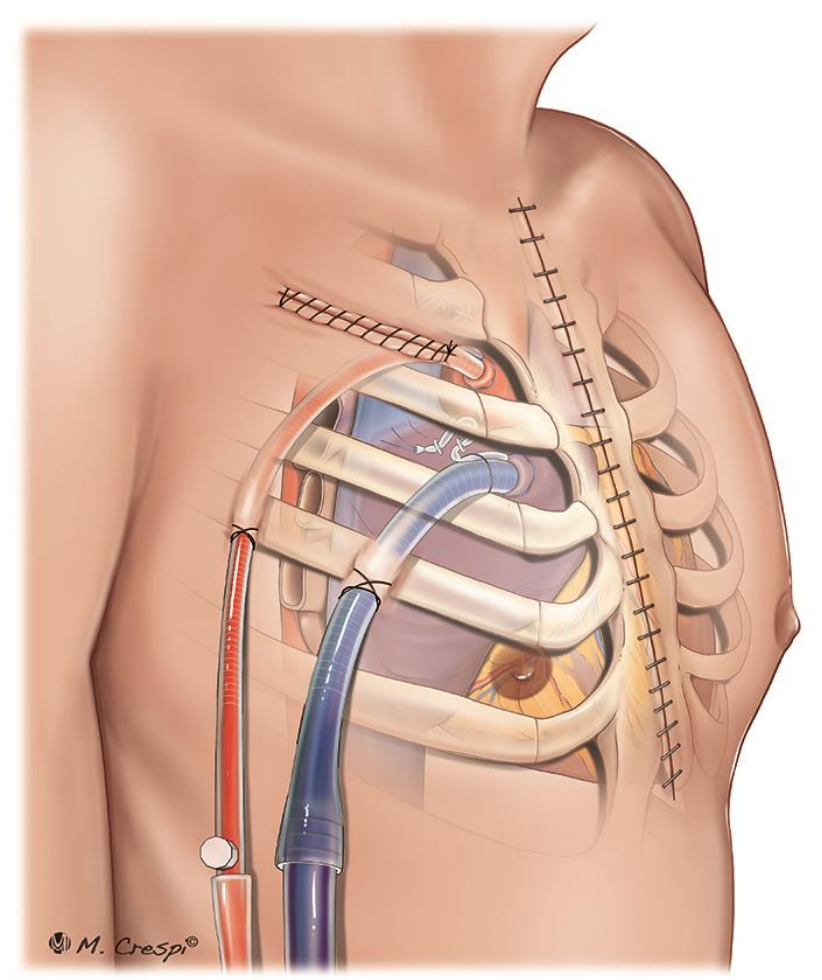

Figure 5. Post-cardiotomy ECMO approaches for cannulation: central cannulation with access through a left mini-thoracotomy, avoiding median sternotomy. 
Table 1 Baseline Chascteristics of Patients Supported by Extracorporeal Membrane Oxygenation (ECMO) Oygenation

\begin{tabular}{|c|c|c|c|c|}
\hline $\begin{array}{l}\text { Author momo - } \\
\text { Year of publication }\end{array}$ & $\begin{array}{l}\text { Patient } \\
\mathrm{N} \\
\text { (Male/female) }\end{array}$ & $\begin{array}{l}\text { Prevalence of } \\
\text { ECMO use, } \%\end{array}$ & $\begin{array}{l}\text { Patient Age Mean } \\
\text { in yearst SD } \\
\text { (Range) }\end{array}$ & $\begin{array}{l}\text { Pre-ECro } \\
\text { Candiac surgery procedures }\end{array}$ \\
\hline Magovern" 1994 & $\begin{array}{l}21 \\
(17 / 4)\end{array}$ & 0.7 & $\begin{array}{l}61.6 \pm 2.2 \\
(33-78)\end{array}$ & $\begin{array}{l}\text { CABG } 66.6 \% \\
\text { MV } 14.3 \% \\
\text { Others } 19.1 \%\end{array}$ \\
\hline Kodera ${ }^{25}-1996$ & $\begin{array}{l}17 \\
(11 / 6)\end{array}$ & na & $\begin{array}{l}41.4 \\
(22-64)\end{array}$ & $\begin{array}{l}\text { CABG } 17.6 \% \\
\text { Valve } 82.4 \%\end{array}$ \\
\hline fiset ${ }^{25}-2001$ & $\begin{array}{l}51 \\
(29 / 22)\end{array}$ & 0.9 & $\begin{array}{l}61 \pm 1.7 \\
(32-77)\end{array}$ & $\begin{array}{l}\text { CABG } 66.6 \% \\
\text { Valve } 9.8 \% \\
\text { CABG + Valve } 9.8 \% \\
\text { HTX } 13.7 \%\end{array}$ \\
\hline Smedira ${ }^{17}-2001$ & $\begin{array}{l}107 \\
(77 / 30)\end{array}$ & 0.5 & $\begin{array}{l}\text { na } \\
(18-82)\end{array}$ & $\begin{array}{l}\text { CABG } 56 \% \\
\text { Valive } 14 \% \\
\text { CABG + Valve } 18 \% \\
\text { Aorta (desc) } 4 \% \\
\text { Cong. } 7 \% \\
\text { LV resection } 1 \%\end{array}$ \\
\hline$K_{0}=2002$ & $\begin{array}{l}76 \\
(48 / 28)\end{array}$ & 2.60 & $\begin{array}{l}56.8 \pm 15.9 \\
(\mathrm{na})\end{array}$ & $\begin{array}{l}\text { CABG } 47.7 \% \\
\text { Valve } 18.4 \% \\
\text { CABG + Valve } 7.9 \% \\
\text { HTx } 15.7 \% \\
\text { Cong. } 3.9 \% \\
\text { Ao Diss. } 2.7 \%\end{array}$ \\
\hline Zhang ${ }^{20}-2006$ & $\begin{array}{l}32 \\
(18 / 14)\end{array}$ & na & $\begin{array}{l}55.4 \pm 11.9 \\
(30-75)\end{array}$ & $\begin{array}{l}\text { CABG } 15.6 \% \\
\text { Valve } 31.2 \% \\
\text { CABG + Valve } 31.2 \% \\
\text { CABG + LVAn. } 6.2 \% \\
\text { PEA } 6.2 \% \\
\text { Others } 6.2 \%\end{array}$ \\
\hline Balitiay ${ }^{20}-2007$ & $\begin{array}{l}45 \\
(35 / 10)\end{array}$ & 0.8 & $\begin{array}{l}60.1 \pm 13.6 \\
(n)\end{array}$ & $\begin{array}{l}\text { CABG } 4.4 \% \\
\text { Valve } 4.4 \% \\
\text { CABG + Valve } 17.8 \% \\
\text { CABG + VSD } 6.7 \% \\
\text { HTX } 4.4 \% \\
\text { LVAD } 11.1 \% \\
\text { Others } 11.9 \%\end{array}$ \\
\hline $\mathrm{Hed}^{21}-2010$ & $\begin{array}{l}51 \\
(36 / 15)\end{array}$ & 2.9 & $\begin{array}{l}63 \pm 15.7 \\
(n o)\end{array}$ & $\begin{array}{l}\text { CABG } 52.9 \% \\
\text { Valve } 21.6 \% \\
\text { CABG + Valve } 13.7 \% \\
\text { HTX } 7.8 \% \\
\text { Others } 3.9 \%\end{array}$ \\
\hline Restant $22-2010$ & $\begin{array}{l}517 \\
(370 / 147)\end{array}$ & 1.28 & $\begin{array}{l}63.5 \pm 11.2 \\
(18-80)\end{array}$ & $\begin{array}{l}\text { CABG } 37.4 \% \\
\text { Valve } 14.3 \% \\
\text { CABG + Valve } 16.8 \% \\
\text { Thor. Tx } 6.5 \% \\
\text { Others } 25 \%\end{array}$ \\
\hline Elsharkamy ${ }^{21}-2010$ & $\begin{array}{l}239 \\
(163 / 76)\end{array}$ & 0.58 & $\begin{array}{l}59.7 \text { (median) } \\
(\mathrm{na})\end{array}$ & $\begin{array}{l}\text { CABG } 4.3 \% \\
\text { Valve } 33.6 \%\end{array}$ \\
\hline Pokersnik -2012 & $\begin{array}{l}49 \\
(33 / 16)\end{array}$ & na & $\begin{array}{l}65 \pm 13 \\
(n a)\end{array}$ & na \\
\hline Mikus $25-2013$ & 14 & 0.25 & $\begin{array}{l}53.1 \pm 14.3 \\
(25-70)\end{array}$ & $\begin{array}{l}\text { CABG } 35.7 \% \\
\text { Valve } 42.9 \% \\
\text { Aortic Root } 21.4 \%\end{array}$ \\
\hline Slott $\operatorname{csch}^{25}-2013$ & $\begin{array}{l}n \\
(59 / 18)\end{array}$ & na & $\begin{array}{l}60 \pm 13 \\
(25-83)\end{array}$ & $\begin{array}{l}\text { CABG } 55.8 \% \\
\text { Valve } 13 \% \\
\text { CABG + Valve } 14.3 \% \\
\text { Aota } 6.5 \% \\
\text { HTx } 2.6 \% \\
\text { Others } 7.8 \% \\
\text { (RedoSungey } 19.5 \% \text { ) }\end{array}$ \\
\hline
\end{tabular}


Table 1 (Continued)

\begin{tabular}{|c|c|c|c|c|}
\hline $\begin{array}{l}\text { Author merance }- \\
\text { Year of publication }\end{array}$ & $\begin{array}{l}\text { Patient } \\
\text { N } \\
\text { (Male/temale) }\end{array}$ & $\begin{array}{l}\text { Prevalence of } \\
\text { ECMO use, } \%\end{array}$ & $\begin{array}{l}\text { Patient Age Mean } \\
\text { in yeas } \pm S D \\
\text { (Range) } \\
\end{array}$ & $\begin{array}{l}\text { Pre-ECM0 } \\
\text { Cardiacsurgery procedures }\end{array}$ \\
\hline Unas $x_{n a s}^{27}-2013$ & $\begin{array}{l}47 \\
(35 / 12)\end{array}$ & na & $\begin{array}{l}64.4 \pm 12.5 \\
(22.83)\end{array}$ & $\begin{array}{l}\text { CABG } 40.4 \% \\
\text { Valve } 17 \% \\
\text { Valve + CABG } 4.2 \% \\
\text { Valve + Aorta } 2 \% \\
\text { Aorta } 10.6 \% \\
\text { Aorta + CABG } 6.4 \% \\
\text { Aortic Root } 4.25 \% \\
\text { VSD } 10.6 \% \\
\text { PEA } 4.25 \%\end{array}$ \\
\hline Ariyaratnam ${ }^{3 n}-2016$ & $\begin{array}{l}14 \\
(8 / 6)\end{array}$ & na & $\begin{array}{l}65.6 \\
(n a)\end{array}$ & $\begin{array}{l}\text { Valve+ CABG } 42.8 \% \\
\text { Aartic Root } 14.3 \% \\
\text { VSD } 7 \% \\
\text { MVR + Maze } 7 \% \\
\text { Ao Diss. } 7 \% \\
\text { Aorta+ MA 7\% } \\
\text { VSD+CABG7\% } \\
\text { CABG + Lobectomy } 7 \%\end{array}$ \\
\hline $\mathrm{Li}^{20}-2015$ & $\begin{array}{l}123 \\
(81 / 42)\end{array}$ & 0.9 & $\begin{array}{l}51.0 \pm 12.2 \\
(7085)\end{array}$ & $\begin{array}{l}\text { CABG } 35.8 \% \\
\text { Valve } 32.5 \% \\
\text { CABG + Vatve } 9.8 \% \\
\text { CABG LVAn. } 2.4 \% \\
\text { HTx } 9 \% \\
\text { Others } 10.5 \%\end{array}$ \\
\hline Sexen $a^{20}-2015^{2}$ & $\begin{array}{l}45 \\
(31 / 14)\end{array}$ & na & $\begin{array}{l}76.8 \pm 4.6 \\
(70-85)\end{array}$ & na \\
\hline Khosandi ${ }^{31}-2016$ & $\begin{array}{l}16 \\
(12 / 4)\end{array}$ & na & $\begin{array}{l}71 \\
(3483)\end{array}$ & no \\
\hline Distelmaier ${ }^{12}-2016^{3}$ & $\begin{array}{l}385 \\
(271 / 114)\end{array}$ & 3.65 & $\begin{array}{l}65 \text { (matian) } \\
(55-72)\end{array}$ & $\begin{array}{l}\text { CABG } 12.5 \% \\
\text { Valve } 28.6 \% \\
\text { CABG + Valve } 21.8 \% \\
\text { HTx } 17.6 \% \\
\text { LVAD } 8 \% \\
\text { Aarta } 6.2 \% \\
\text { Others } 5.2 \%\end{array}$ \\
\hline Mazzeffin $-2016^{3}$ & $\begin{array}{l}23 \\
(14 / 9)\end{array}$ & 0.42 & $\begin{array}{l}57 \pm 15 \\
(3486)\end{array}$ & $\begin{array}{l}\text { CABG } 30.4 \% \\
\text { Valve } 52.2 \% \\
\text { Others } 17.4 \%\end{array}$ \\
\hline Chen $^{16}-2017$ & $\begin{array}{l}1,141 \\
(813 / 328)\end{array}$ & 1.91 & $\begin{array}{l}63.8 \pm 13.2 \\
(n a)\end{array}$ & $\begin{array}{l}\text { CABG } 63.9 \% \\
\text { Vatve } 24.1 \% \\
\text { CABG + Vative } 12 \%\end{array}$ \\
\hline Guihaire $^{23}-2017$ & $\begin{array}{l}92 \\
(53 / 39)\end{array}$ & 0.7 & $\begin{array}{l}64.5 \\
(18-83)\end{array}$ & $\begin{array}{l}\text { CABG } 8.6 \% \\
\text { Vatve } 74.1 \% \\
\text { Ao Diss. } 12.1 \% \\
\text { LVAD } 3.4 \% \\
\text { Others } 1.7 \%\end{array}$ \\
\hline$R a f a^{35}-2017$ & 86 & na & $\begin{array}{l}65 \pm 11.2 \\
(31-86)\end{array}$ & $\begin{array}{l}\text { CABG } 22 \% \\
\text { CABG + Vatve } 21 \% \\
\text { Vatve } 16 \% \\
\text { Ao Diss. } 4 \% \\
\text { Others } 24 \%\end{array}$ \\
\hline$F u x^{21}-2018$ & $\begin{array}{l}105 \\
(80 / 25)\end{array}$ & na & $\begin{array}{l}62 \text { (matian) } \\
(18-77)\end{array}$ & $\begin{array}{l}\text { CABG } 20 \% \\
\text { Single, other than -CABG } 29 \% \\
2 \text { procedures } 31 \% \\
3 \text { procedures } 20 \%\end{array}$ \\
\hline
\end{tabular}

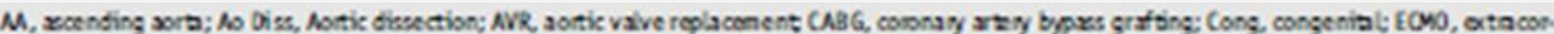

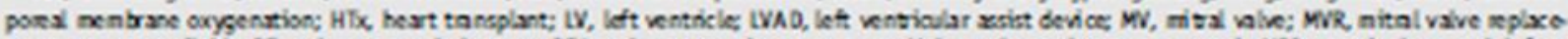

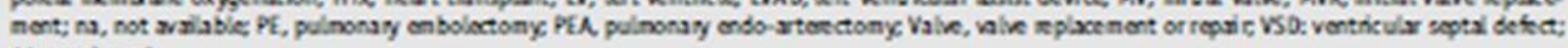
M. sortic arch

Study indudod only prtients over 70yeas of sge

Stuby induded only ortacoporeal ardiopulnonary resuscitation (ECPR) ftzr cardx surgery 


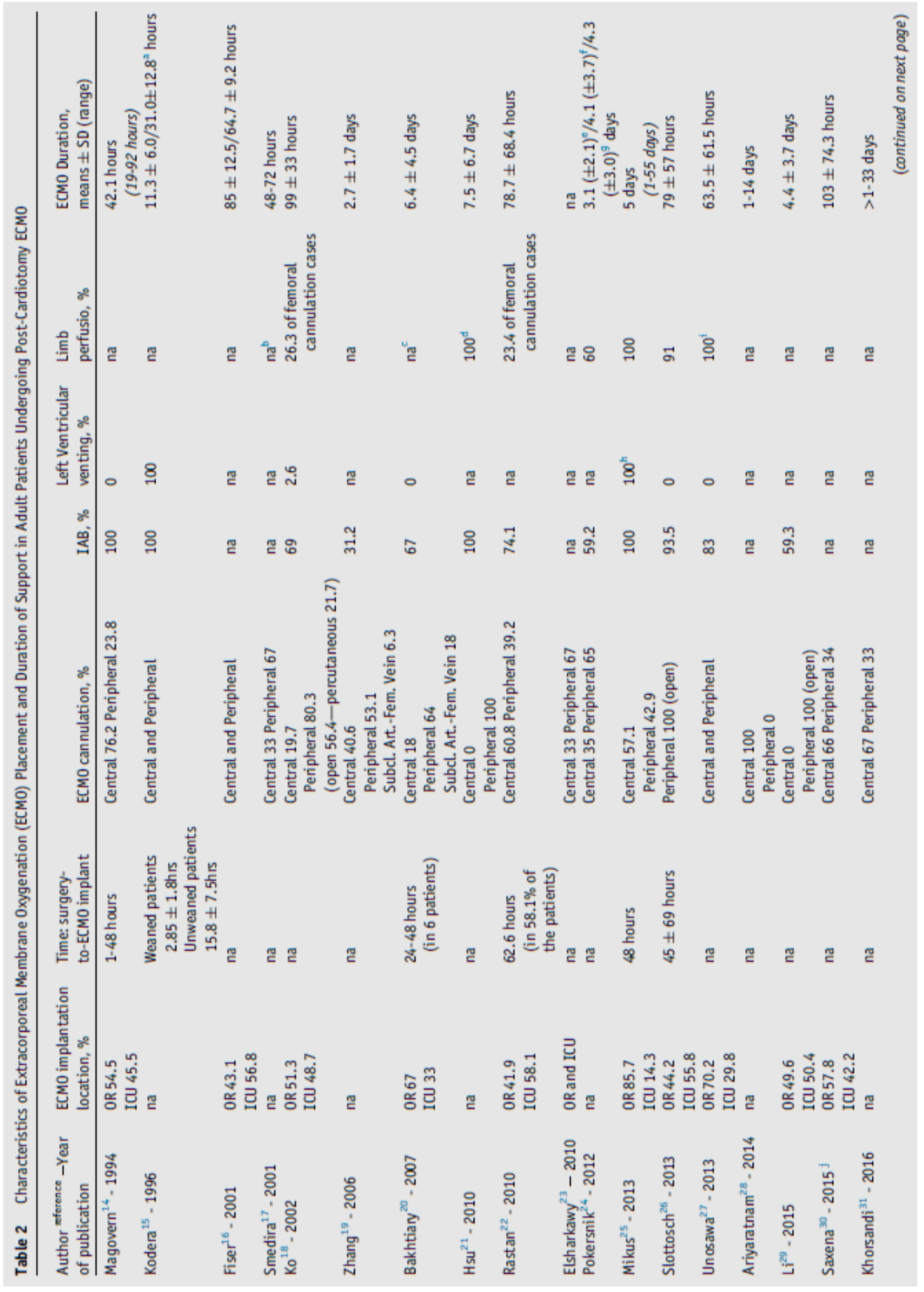




\begin{tabular}{|c|c|c|c|c|c|c|c|}
\hline $\begin{array}{l}\text { Author refe mence -Year } \\
\text { of publication }\end{array}$ & $\begin{array}{l}\text { ECMO implantation } \\
\text { location, \% }\end{array}$ & $\begin{array}{l}\text { Time: surgery- } \\
\text { to-ECMO implant }\end{array}$ & ECMO cannulation, \% & IAB, \% & $\begin{array}{l}\text { Left Ventricular } \\
\text { venting, \% }\end{array}$ & $\begin{array}{l}\text { Limb } \\
\text { perfusio, \% }\end{array}$ & $\begin{array}{l}\text { ECMO Duration, } \\
\text { means } \pm \text { SD (range) }\end{array}$ \\
\hline Distelmaier $^{32}-2016^{k}$ & $\begin{array}{l}\text { OR } 86 \\
\text { ICU } 14\end{array}$ & na & $\begin{array}{l}\text { Aorta-Femoral } 10 \\
\text { Subcl-Fem } 38 \\
\text { Peripheral } 52\end{array}$ & 12 & na & na & 96 hours \\
\hline $\begin{array}{l}\text { Mazzeffi }^{33}-2016^{k} \\
\text { Chen }^{34}-2017\end{array}$ & $\begin{array}{l}\mathrm{OR} \text { and ICU } \\
\text { na }\end{array}$ & $31(15-52) \mathrm{min}$. & $\begin{array}{l}\text { Central } 60.9 \text { Peripheral } 39.1 \\
\text { na }\end{array}$ & $\begin{array}{l}13 \\
\text { na }\end{array}$ & $\begin{array}{l}0 \\
\text { na }\end{array}$ & na & $\begin{array}{l}\text { 0-14 days } \\
\text { na }\end{array}$ \\
\hline Guihaire ${ }^{35}-2017$ & $\begin{array}{l}\text { OR } 86.9 \\
\text { ICU } 13.1\end{array}$ & 48 hours & $\begin{array}{l}\text { Central } 14.7 \\
\text { Peripheral } 85.3\end{array}$ & 27.1 & 14.1 & na & $\begin{array}{l}6 \text { days } \\
(1-28 \text { days })\end{array}$ \\
\hline Raffa $^{36}-2017$ & $\begin{array}{l}\text { OR } 55.8 \\
\text { ICU } 44.2\end{array}$ & na & $\begin{array}{l}\text { Central } 17.4 \\
\text { Peripheral } 65.1 \\
\text { Central (artery)-Peripheral } \\
\text { (vein) } 17.4\end{array}$ & 27.1 & na & na & 5 days \\
\hline $\mathrm{Fux}^{37}-2018$ & OR 49 & na & $\begin{array}{l}\text { Peripheral } 76 \\
\text { Central } 24\end{array}$ & na & na & $90 \%$ & $\begin{array}{l}7 \text { days (median) } \\
(1-55)\end{array}$ \\
\hline \multicolumn{8}{|c|}{$\begin{array}{l}\text { CPB, cardiopulmonary bypass; EOM0, extracorporeal membrane oxygenation; ECPR, extracorporeal cardiopulmonary resuscitation; IABP, intra-aortic balloon pump; ICU, intensive care unit; na, not available; OR, } \\
\text { operating room; RVF, right ventricular failure; Subd-Fem, subclavian artery }\end{array}$} \\
\hline \multicolumn{8}{|c|}{$\begin{array}{l}\text { "Groups related to patients who were weaned and discharged vers us patients weaned from ECMO but not discharged and died in hospital } \\
\text { "Performed in all patients with femoral cannulation in the most recent series, after an initial experience without it characterized by ischemia episodes of the cannulated limb } \\
\text { "Distal limb perfusion performed but no data available } \\
\text { "Distal limb perfusion achieved by cannulating the femoral artery with a } 8 \mathrm{~mm} \text {-graft perfusing antegradely and retrogradely } \\
\text { "Group of patients receiving Biomedicus (Medtronic Inc, Minneapolis, Minn) pump and Affinity oxygenator (Medtronic Inc. Minneapolis, Minn) } \\
\text { "Group of patients receiving Biomedicus (Medtronic Inc, Minneapolis, Minn) and Quadrox D (Getinge, Hirrlingen, Germany) oxygenator } \\
\text { "Group of patients receiving Rotaflow (Getinge, Hirrlingen, Germany) pump and Quad rox D (Getinge, Hirrtingen, Germany) oxygenator } \\
\text { "Venting was performed in all patients who had central cannulation and no patients with femoral cannulation } \\
\text { 'Distal limb perfusion was performed in } 100 \% \text { of cases after } 1999 \\
\text { 'Study included only patients over } 70 \text { years of age }\end{array}$} \\
\hline
\end{tabular}

\begin{tabular}{|c|c|c|c|c|c|c|c|}
\hline $\begin{array}{l}\text { Author }{ }^{\text {reterence }} \text {-Year } \\
\text { of publication }\end{array}$ & $\begin{array}{l}\text { Bleeding. } \\
\%\end{array}$ & $\begin{array}{l}\text { ECM0 system } \\
\text { failure, \% }\end{array}$ & $\begin{array}{l}\text { Liver } \\
\text { failure, \% }\end{array}$ & $\begin{array}{l}\text { Sepsis/infection/ } \\
\text { bacteremia, \% }\end{array}$ & $\begin{array}{l}\text { CNS } \\
\text { events, } \%\end{array}$ & $\begin{array}{l}\text { Kidney } \\
\text { failure, \% }\end{array}$ & $\begin{array}{l}\text { Limb ischemia } \\
\text { (Including } \\
\text { amputation } \\
\text { or fasciotomy), \% }\end{array}$ \\
\hline Magovern $^{14}-1994$ & 91 & 33 (oxygenator change) & na & 33 & 28.5 & 19 & 4.7 \\
\hline Kodera $^{15}$ - 1996 & $\mathrm{Na}$ & na & na & na & na & na & na \\
\hline Fiser $^{16}-2001$ & $\mathrm{Na}$ & na & na & 7 & 28 & 4 & na \\
\hline Smedira ${ }^{17}-2001$ & $\mathrm{Na}$ & 5 & na & 48 & 29 & 39 & 27 \\
\hline $\mathrm{Ko}^{18}-2002$ & 46 & na & na & 26 & 11.8 & na & 17.1 \\
\hline Zhang $^{19}-2006$ & $\mathrm{Na}$ & na & na & na & na & na & na \\
\hline Bakhtiary $^{20}-2007$ & 87 & 9 & na & 58 & 9 & 87 & 22 \\
\hline $\mathrm{Hsu}^{21} \cdot 2010$ & 2.9 & na & na & 34 & 29 & 75 & 5.9 \\
\hline Rastan $^{22}-2010$ & 58 & na & na & 24.8 & 17.4 & 65 & 19.9 \\
\hline Elsharkawy ${ }^{23}-2010$ & na & na & na & 36 & na & na & na \\
\hline Pokersnik $^{24}-2012$ & 71 & $\begin{array}{l}63.6^{3} \\
0^{6} \\
7.4^{e}\end{array}$ & na & 32.6 & 6.1 & 32.6 & na \\
\hline Mikus $^{25}-2013$ & 64.3 & 7.1 & na & 42.8 & 0 & 50 & 0 \\
\hline Slottosch ${ }^{26}-2013$ & 29.9 & na & 3.9 & 29 & 22.1 & 68.8 & 20.8 \\
\hline Unosawa $^{27}-2013$ & na & na & na & na & 12.7 & 17 & 10.6 \\
\hline Ariyaratnam ${ }^{28}-2014$ & na & na & na & na & na & na & na \\
\hline $\mathrm{Li}^{\mathrm{3}}-2015$ & na & na & na & 22.3 & $6.2^{4}$ & 39.2 & 29.9 \\
\hline Saxena ${ }^{\circ}-2015^{\circ}$ & na & na & na & 24.4 & 4.4 & 44.4 & 13.3 \\
\hline Khorsandi ${ }^{11}-2016$ & na & na & na & 12.5 & 18.7 & 18.7 & 12.5 \\
\hline Distelmaier ${ }^{12}-2016^{6}$ & na & na & na & na & na & na & na \\
\hline Mazzeffi ${ }^{33}-2016^{4}$ & 6.2 & na & na & 18.8 & 25 & na & na \\
\hline Chen $^{34}-2017$ & 11.3 & na & na & 13.2 & 4.3 & 32.9 & 2.3 \\
\hline Guihaire $^{35}-2017$ & 19.5 & na & na & 52.1 & 3.2 & na & 9.8 \\
\hline $\mathrm{Raffa}^{36}-2017$ & 46.4 & na & na & 21.4 & 29.7 & 29.8 & 10.7 \\
\hline $\mathrm{Fux}^{37}-2018$ & 68 & na & na & 24 & $\begin{array}{l}\text { intracranial hemorrhage } 7 \\
\text { stroke } 16 \\
\text { brain death } 8\end{array}$ & 70 & $\begin{array}{l}\text { leg fasciotomy } 7 \\
\text { leg amputation } 1\end{array}$ \\
\hline $\begin{array}{l}\text { Abbreviations: ARF, act } \\
\text { phosphokinase; CPR, ardi } \\
\text { loon pump; LV, left ventn } \\
\text { device, CNS. central nervo } \\
\text { "Group of patients rece } \\
\text { Eroup of patients rece } \\
\text { Group of patients rece } \\
\text { Enly stroke reported } \\
\text { "Study induded only pa } \\
\text { Study induded only ex }\end{array}$ & $\begin{array}{l}\text { LVAD, left ver } \\
\text { stem } \\
\text { Biomedicus } \\
\text { Biomedicus } \\
\text { Rotaflow (Get } \\
\text { ss over } 70 \text { yea } \\
\text { iporeal cardic }\end{array}$ & $\begin{array}{l}\text { ar assist device; LVEF, left ve } \\
\text { nic Inc, Minneapolis, Minn) p } \\
\text { nic Inc, Minneapolis, Minn) a } \\
\text { Hirnlingen, Germany) pump an } \\
\text { nary resuscitation (ECPR) afte }\end{array}$ & $\begin{array}{l}\text { ar jection fr. } \\
\text { Id Affinity ox } \\
\text { ad rox } D \text { (Getin } \\
\text { drox } D \text { (Geting } \\
\text { ac surgery }\end{array}$ & $\begin{array}{l}\text { 36, coronary artery byp } \\
\text { pus hemofiltration; ECM } \\
\text { : Mof, multiongan failt } \\
\text { or (Mectronic Inc. Min } \\
\text { mtingen, Germany) oxy } \\
\text { flingen, Germany) oxys }\end{array}$ & $\begin{array}{l}\text { fting; OSS, central nervous syst } \\
\text { acorporeal membrane oxygenat } \\
\text { not available; PA, pulmonary } \\
\text { lis, Minn) } \\
\text { or }\end{array}$ & $\begin{array}{l}\text { B, cart iopulm } \\
x \text {, heart trans } \\
\text { VA, veno-art }\end{array}$ & $\begin{array}{l}\text { bypass; CPK, creatine- } \\
\text { IABP, intra-aortic bal- } \\
\text { VAD, ventricular assist }\end{array}$ \\
\hline
\end{tabular}




\begin{tabular}{|c|c|c|c|c|c|c|}
\hline $\begin{array}{l}\text { Author }{ }^{\text {Atronese }} \text {-Year } \\
\text { of Publication }\end{array}$ & Weaning, \% & $\begin{array}{l}\text { In-Hospital } \\
\text { Survival, \% }\end{array}$ & $\begin{array}{l}\text { Bridge to LVAD/HTx, } \\
\text { n/ total patients (\%) } \\
\%\end{array}$ & $\begin{array}{l}\text { In-Hospital } \\
\text { Survival LVAD/HTx } \\
\text { after ECMO, n/total patients (\%) } \\
\text { (\% }\end{array}$ & $\begin{array}{l}\text { 1-Year } \\
\text { Survival, \% }\end{array}$ & Predictors of In-Hospital Mortality \\
\hline Magovern $^{14}-1994$ & 76 & 52 & None & $\cdot$ & 47.6 & $\begin{array}{l}\text { Mitral valve surgery } \\
\text { Non-convertible ventricular fibrillation }\end{array}$ \\
\hline Kodera $^{15}-1996$ & 70.6 & 47.1 & None & $\cdot$ & $\begin{array}{l}\text { na } \\
\text { (30.9 at } 5 \text { years) }\end{array}$ & EOMO duration $>60$ hours \\
\hline Fiser $^{16}-2001$ & 31 & 16 & na & na & na & $\begin{array}{l}\text { Age }>65 \text { yrs., LVEF }<30 \% \text { after } 48 \text { hours on ECMO } \\
\text { Prolonged CPR ( }>5 \text { minutes) prior to ECMO implant }\end{array}$ \\
\hline Smedira ${ }^{17}-2001$ & 39.2 & 33,3 & $\begin{array}{l}\text { HTx } 0(0 \%) \\
\text { LVAD: } 18 / 107(16.8 \%)\end{array}$ & $\begin{array}{l}\text { HTx -LVAD: } 13 / 18(72 \%) \\
\text { at 1-year follow-up }\end{array}$ & na & na \\
\hline $\mathrm{Ko}^{18}-2002$ & 60.5 & 26.3 & $\begin{array}{l}\text { HTx: } 2 / 76(2.6 \%) \\
\text { LVAD: } 2 / 76(2.6 \%)\end{array}$ & $\begin{array}{l}\text { HTx: 0/2 (0\%) } \\
\text { LVAD: } 1 / 2(50 \%)\end{array}$ & 45.4 & ARF requiring dialysis on ECMO Peak CPK (U/L) $)^{2}$ \\
\hline Zhang $^{10}-2006$ & 43.7 & 25 & na & na & na & $\begin{array}{l}\text { Blood lactate level } 48 \text { hours after ECMO initiation } \\
\text { MB-isoenzyme } 48 \text { hours after ECMO initiation } \\
\text { CK-MB relative index ( } C K-M B / \text { total CK) } 48 \text { hours after ECMO initiation }\end{array}$ \\
\hline Bakhtiary ${ }^{20}-2007$ & 55.5 & 29 & $\begin{array}{l}\text { HTx: } 2 / 45(4 \%) \\
\text { LVAD: } 5 / 45(11 \%)\end{array}$ & $\begin{array}{l}\text { HTx: } 1 / 2(50 \%) \\
\text { LVAD: } 3 / 5(60 \%) \\
\text { at 30-day follow-up }\end{array}$ & 29 & $\begin{array}{l}\text { PA hypertension } \\
\text { Diabetes } \\
\text { Lack of IABP }\end{array}$ \\
\hline $\mathrm{Hsu}^{21}-2010$ & 53 & 24.8 & $\begin{array}{l}\text { HTx: } 4 / 51(8 \%) \\
\text { LVAD:- }\end{array}$ & $\begin{array}{l}\text { HTx. } 3 / 4 \text { (75\%) } \\
\text { at } 30 \text {-day follow-up; } 1 / 4 \text { (25\%) } \\
\text { at } 1 \text {-year follow-up. } \\
\text { LVAD:- }\end{array}$ & 16.5 & $\begin{array}{l}\text { Low peri-op serum albumin level } \\
\text { Platelet count } \\
\text { Oxygen press ure of the venous tube of the EOMO Poor cardiac systolic } \\
\text { function }\end{array}$ \\
\hline Rastan $^{22}-2010$ & 63.3 & 30,4 & $\begin{array}{l}\text { HTx: 5/517(1\%) } \\
\text { LVAD: } 15 / 517(3 \%)\end{array}$ & $\begin{array}{l}\text { HTx: } 2 / 5(40 \%) \\
\text { LVAD: } 3 / 15(20 \%)\end{array}$ & na & $\begin{array}{l}\text { Age }>70 \text {, Diabetes, Obesity, Preoperative CRF, Operative lactate } \\
>4 \text { mmo /l, Logistic Euroscore }>20\end{array}$ \\
\hline Elsharkawy ${ }^{23}-2010$ & na & 16 & na & na & na & $\begin{array}{l}\text { Older age } \\
\text { Higher preoperative albumin } \\
\text { Diabetes } \\
\text { CABG surgery } \\
\text { Longer CPB time } \\
\text { Cardiogenic shock at time of VA-ECMO implant }\end{array}$ \\
\hline Pokersnik ${ }^{24}-2012$ & $\begin{array}{l}54.9\left(63.6^{b} /\right. \\
\left.45.5^{4} / 55.6^{4}\right)\end{array}$ & 36 & HTx: - LVAD: $2 / 33(6 \%)$ & HTx - LVAD: $0 / 2(0 \%)$ & na & Older age \\
\hline Mikus $^{25}-2013$ & 50 & 42.8 & None & - & na & High transfusion rate \\
\hline Slottosch ${ }^{26}-2013$ & 62 & 32.6 & $\begin{array}{l}\text { HTx: } 2 / 77(2.6 \%) \\
\text { VAD: - }\end{array}$ & $\begin{array}{l}\text { HTx } 0 / 2(0 \%) \\
\text { VAD:- }\end{array}$ & na & $\begin{array}{l}\text { Older age } \\
\text { Greater lactate after } 24 \text { hours of ECMO } \\
\text { Longer ECMO duration } \\
\text { ECMO related or gastrointestinal complications }\end{array}$ \\
\hline Unosawa $^{27}-2013$ & 61.7 & 40.3 & na & $\cdot$ & 24.5 & $\begin{array}{l}\text { Older age } \\
\text { Incomplete sternal closure } \\
\text { ECMO support }>48 \text { hours } \\
\text { Dialysis for ARF } \\
\text { MOF } \\
\text { Brain injury } \\
\text { Peak } \mathrm{Cr}(\mathrm{mg} / \mathrm{dl}) \text { during ECMO }\end{array}$ \\
\hline Ariyaratnam $^{28}-2014$ & 50 & 31.2 & na & - & na & Increase requirements for vasoconstrictors \\
\hline $\mathrm{Li}^{25}-2015$ & 56 & 34.1 & na & $\cdot$ & na & $\begin{array}{l}\text { Age } \\
\text { Gender (Female) } \\
\text { Mean lactate concentration (at } 6 \text { and } 12 \text { hours from ECMO initiation) } \\
\text { Lactate clearance (at } 6 \text { and } 12 \text { hours from ECMO initiation) }\end{array}$ \\
\hline Saxena ${ }^{30}-2015^{\circ}$ & 53.4 & 24.4 & None & - & na & $\begin{array}{l}\text { Preoperative atrial fibrillation } \\
\text { CWH } \\
\text { Lactic acidos is on ECMO } \\
\text { Persistent coagulopathy on EOMO } \\
\text { Delayed ECMO implant } \\
\text { MOF in patients } 50-69 \\
\text { High postop AST }\end{array}$ \\
\hline Khorsandi ${ }^{31}-2016$ & na & 51 & na & - & 29.8 & $\begin{array}{l}\text { Older age } \\
\text { Emergent nature of surgery } \\
\text { Pre-existing preoper ative severe LV impairment }\end{array}$ \\
\hline Distelmaier $^{32}-2016^{f}$ & na & 32 & na & - $\quad 2$ & na & na \\
\hline Mazzeffi ${ }^{33}-2016^{t}$ & na & 30.1 & HTx: - LVAD: $2 / 23(8.7 \%)$ & HTx: - LVAD: na & na & Age $>50$ years, Cause of cardiac arrest except tamponade \\
\hline $\mathrm{Chen}^{34}-2017$ & na & 38.3 & na & na & 24.1 & $\begin{array}{l}\text { ARF } \\
\text { Massive blood transfusion }\end{array}$ \\
\hline Guihaire $^{35}-2017$ & 48 & 42 & $\begin{array}{l}\text { HTx: } 2 / 92(2.17 \%) \\
\text { LVAD: } 2 / 92(2.17 \%)\end{array}$ & $\begin{array}{l}\text { HTx: } 2 / 2(100 \%) \\
\text { LVAD: } 1 / 2(50 \%)\end{array}$ & 39 & $\begin{array}{l}\text { Age } \\
\text { Valvular surgery } \\
\text { Peak lactate level at } 24 \mathrm{hrs}\end{array}$ \\
\hline Raffa $^{36}-2017$ & 49.4 & 37 & None & - & na & $\begin{array}{l}\text { Age }>65 \text { years } \\
\text { Postoperative Arrtythmias }\end{array}$ \\
\hline Fux $^{37}-2018$ & 51 & 56 & $\begin{array}{l}\text { HTx: } 1 / 105(0.9 \%) \\
\text { LVAD: } 1 / 105(0.9 \%)\end{array}$ & na & na & $\begin{array}{l}\text { Arterial lactate } \\
\text { Ischemic heart disease }\end{array}$ \\
\hline $\begin{array}{l}\text { AST: aspartate transan } \\
\text { ure; CWH: continuous ve } \\
\text { MOF: multiorgan fa ilure; }\end{array}$ & & lany artery: & $\begin{array}{l}\text { al membrane oxygenation; } \\
\text { no-artenial; VAD: ventricule }\end{array}$ & $\begin{array}{l}\text { hearttansplant; IABP: int } \\
\text { ist device }\end{array}$ & marb & $\begin{array}{l}\text { tine-phosphokinase; CPR: cardiopulmonary resuscitation; CRF: chronic renal fail- } \\
\text { icle; LVAD; left ventricular assist device; LVEF: left ventricular ejection fraction; }\end{array}$ \\
\hline $\begin{array}{l}\text { "Values in the first } 3 \mathrm{~d} \\
\text { "Group of patients rec } \\
\text { "Group of pattients rec } \\
\text { 'Group of patients rec } \\
\text { "Study included orlyp } \\
\text { 'Study included onlye }\end{array}$ & $\begin{array}{l}\text { ays of ECMO initiation } \\
\text { eiving Biomedicus ( } M \\
\text { eiving Biomedicus (M } \\
\text { eiving Rotaflow (Geti } \\
\text { attients over } 70 \text { years } \\
\text { xtracorporeal cardiop }\end{array}$ & $\begin{array}{l}\text { onic Inc, Minnea, } \\
\text { onic Inc, Minnea, } \\
\text { Hirtuingen, Germ } \\
\text { ge } \\
\text { onary pesuscitatic }\end{array}$ & $\begin{array}{l}\text { olis, Minn) pump and Affinity oxy } \\
\text { bis, Minn) and Quadrox D (Geting } \\
\text { ny) pump and Quadrox D (Geting } \\
\text { (ECPR) after cardiac surgery }\end{array}$ & $\begin{array}{l}\text { enator (Medtronic Inc. Minne apolis, Min } \\
\text { e, Hirrtingen, Germany) oxygenator } \\
\text { Hirrtingen, Germany) axygenator }\end{array}$ & & \\
\hline
\end{tabular}




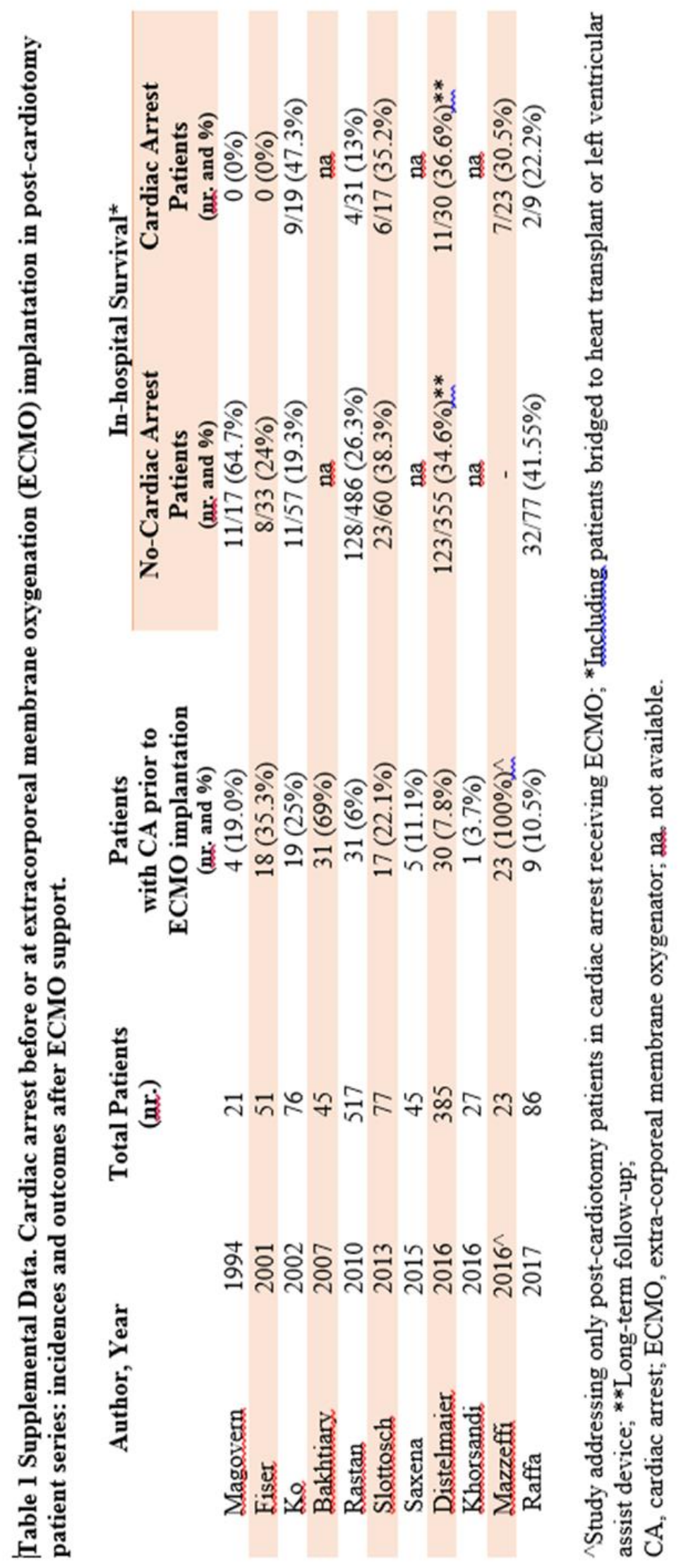




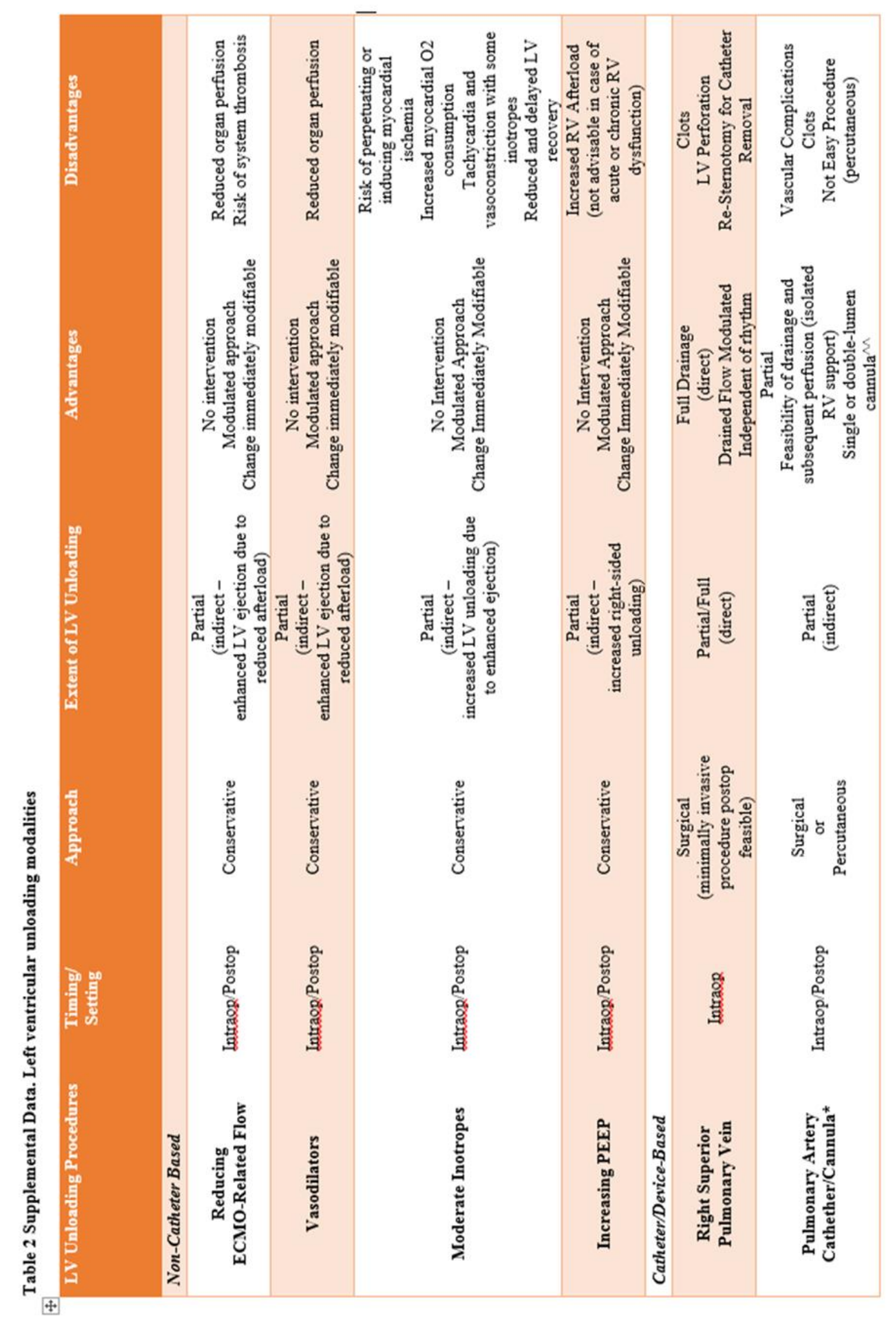




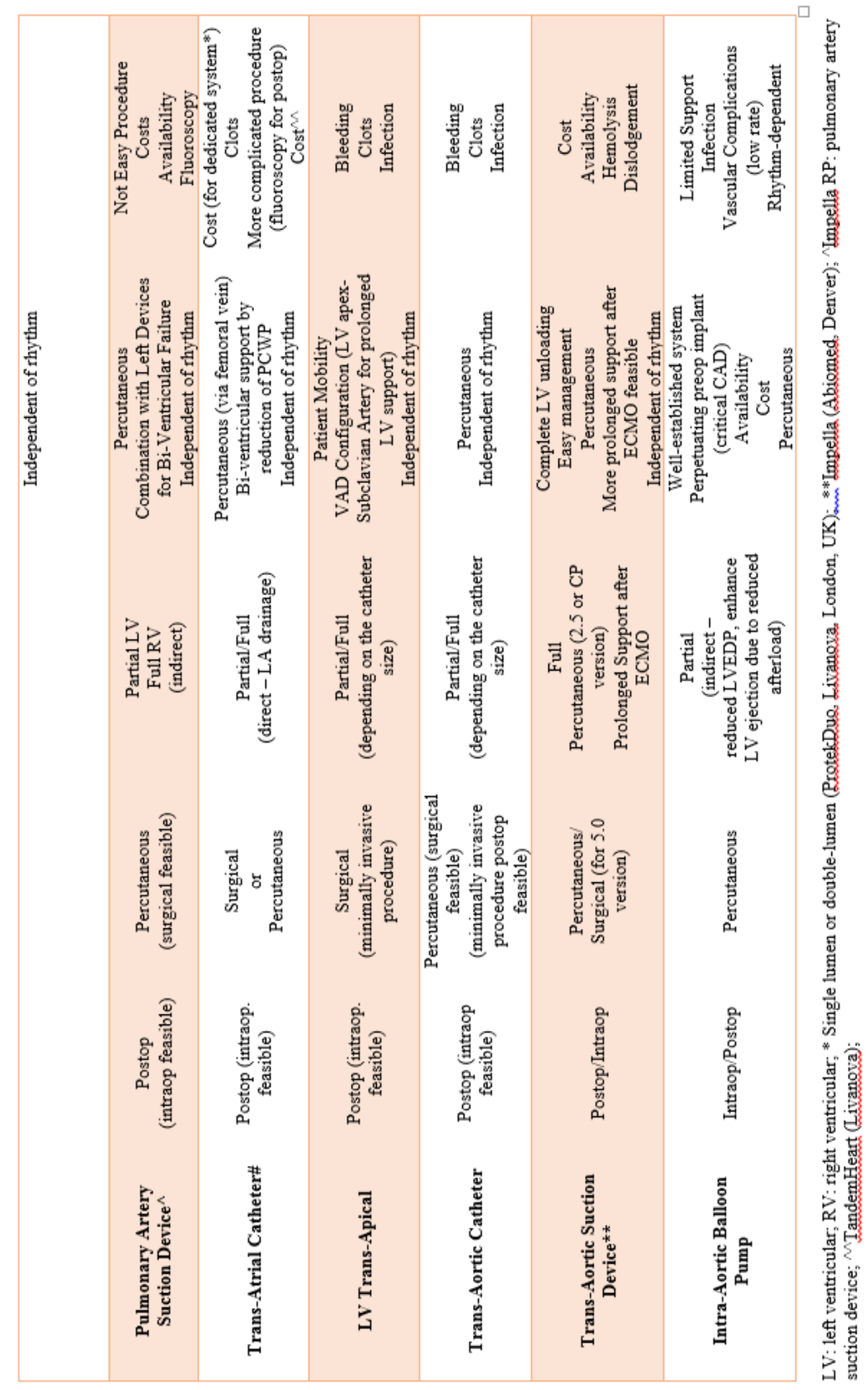




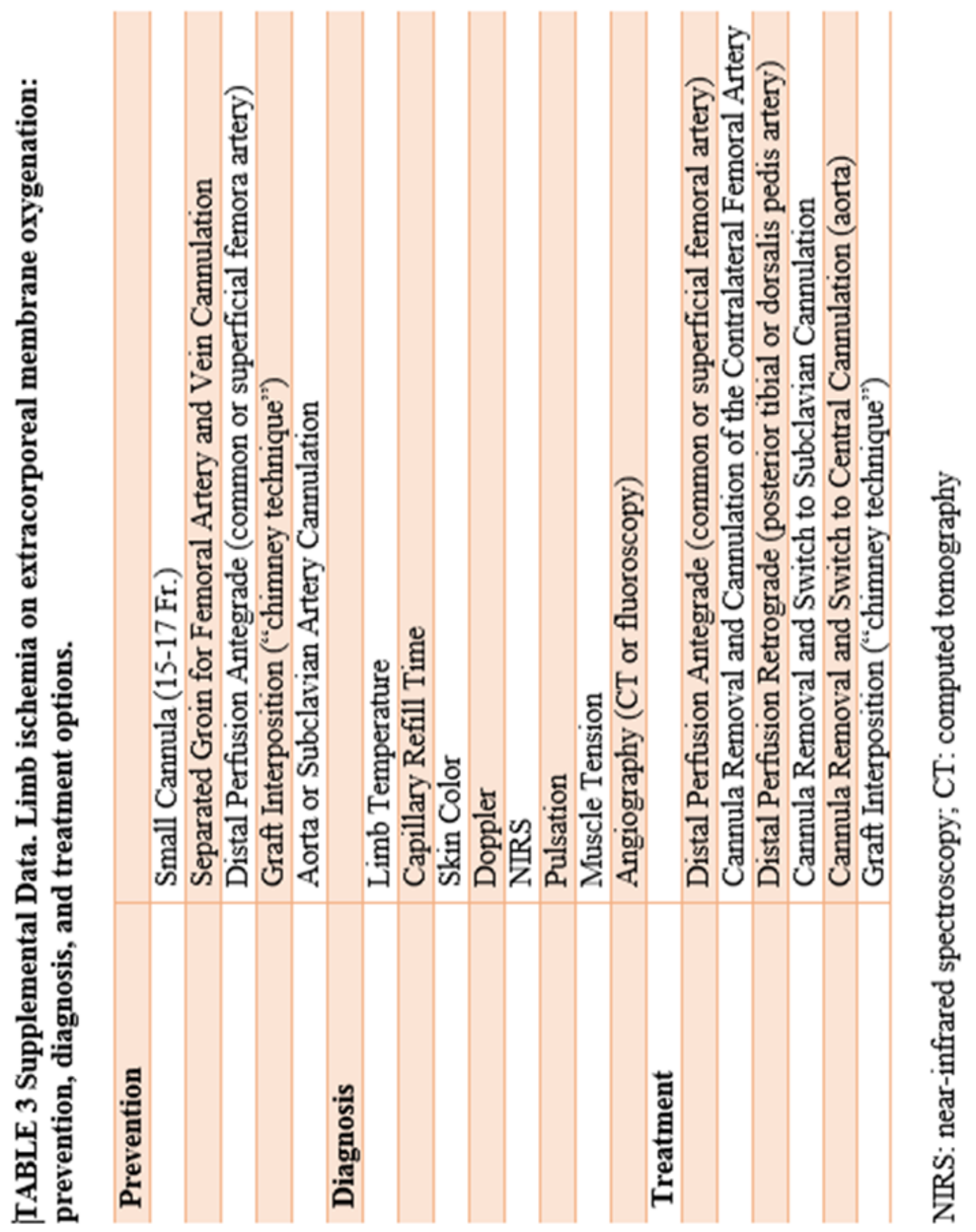




\section{References.}

1. Bellumkonda L, Gul B, Masri SC. Evolving concepts in diagnosis and management of cardiogenic shock. Am J Cardiol 2018;122:1104-10

2. Stretch R, Sauer CM, Yuh DD, Bonde P. National trends in the utilization of short-term mechanical circulatory support. J Am Coll Cardiol 2014;64:1407-15

3. McCarthy FH, McDermott KM, Kini V, et al. Trends in U.S. extracorporeal membrane oxygenation use and outcome: 2002-2012. Sem Thorac Surg 2015;27:81-88

4. Sauer CM, Yuh DD, Bonde P. Extracorporeal membrane oxygenation use increased $433 \%$ in adults in the United States from 2006 to 2011. ASAIO J 2015:61:31-36

5. Maxwell BG, Powers AJ, Sheikh AY, Lee PHU, Lobato RL, Wong JK. Resource use trends in extracorporeal membrane oxygenation in adults: an analysis of the Nationwide Inpatient Sample 1998-2009. J Thorac Cardiovasc Surg 2014;148;416-21

6. Whitman GJR. Extracorporeal membrane oxygenation for the treatment of postcardiotomy shock. J Thorac Cardiovasc Surg 2016;153:95-101

7. Goldstein DJ, Oz MC. Mechanical support for postcardiotomy cardiogenic shock. Sem Thorac Cardiovasc Surg 2000;12:220-8

8. Curtis JJ, McKenney-Knox CA, Wagner-Mann CC. Postcardiotomy centrifugal assist: a single surgeon's experience. Artif Organs 2002;26:994-7

9. Jurmann MJ, Siniawski H, Erb M, Drews T, Hetzer R. Initial experience with miniature axial flow ventricular assist devices for postcardiotomy heart failure. Ann Thorac Surg $2004 ; 77: 1642-7$

10. Hernandez AF, Grab JD, Gammie JS, et al. A decade of short-term outcomes in post-cardiac surgery ventricular assist device implantation. Circulation 2007;116:606-12

11. Akay MH, Gregoric ID, Radovancevic R, Cohn WE, Frazier OH. Timely use of a CentriMag heart assist device improves survival in postcardiotomy cardiogenic shock. J Card Surg 2011;26:548-52 
12. Sylvin EA, Stern DR, Goldstein DJ. Mechanical support for postcardiotomy cardiogenic shock: has progress been made? J Card Surg 2010;25:442-54

13. Fukuhara S, Takeda K, Garan A, et al. Contemporary mechanical circulatory support therapy for postcardiotomy shock. Gen Thorac Cardiovasc Surg 2016;64:183-91

14. Magovern GJ Jr, Magovern JA, Benckart DH, et al. Extracorporeal membrane oxygenation: preliminary results in patients with postcardiotomy cardiogenic shock. Ann Thorac Surg $1994 ; 57: 1462-71$

15. Kodera K, Kitamura M, Hachida M, Endo M, Hashimoto A, Koyanagi H. Biventricular bypass with oxygenation for postcardiotomy ventricular failure. Artif Organs 1996;20:724-7

16. Fiser SM, Tribble CG, Kaza AK, et al. When to discontinue extracorporeal membrane oxygenation for postcardiotomy support. Ann Thorac Surg 2001;71:210-4

17. Smedira NG, Blackstone EH. Postcardiotomy mechanical support: risk factors and outcomes. Ann Thorac Surg 2001;71:S60-6

18. Ko WJ, Lin CY, Chen RJ, Wang SS, Lin FY, Chen YS. Extracorporeal membrane oxygenation support for adult postcardiotomy cardiogenic shock. Ann Thorac Surg 2002;73:538-45

19. Zhang R, Kofidis T, Kamiya $\mathrm{H}$, et al. Creatine kinase isoenzyme MB relative index as predictor of mortality on extracorporeal membrane oxygenation support for postcardiotomy cardiogenic shock in adult patients. Eur J Cardio-Thorac Surg 2006;30:617-20

20. Bakhtiary F, Keller H, Dogan S, et al. Venoarterial extracorporeal membrane oxygenation for treatment of cardiogenic shock : clinical experiences in 45 adult patients. J Thorac Cardiovasc Surg $2008 ; 135: 382-8$

21. Hsu PS, Chen JL, Hong GJ, et al. Extracorporeal membrane oxygenation for refractory cardiogenic shock after cardiac surgery: predictors of early mortality and outcome from 51 patients. Eur J Cardio-Thorac Surg 2010;37:328-33 
22. Rastan AJ, Dege A, Mohr M, et al. Early and late outcome of 517 consecutive patients treated with extracorporeal membrane oxygenation for refractory postcardiotomy cardiogenic shock. J Thorac Cardiovasc Surg 2010;139:302-11

23. Elsharkawy HA, Li L, Skr Esa WA, Sessler D, Bashour CA. Outcome in patients who require venoarterial extracorporeal membrane oxygenation support after cardiac surgery. J CardioThorac Vasc Anesth 2010;24:946-51

24. Pokersnik JA, Buda T, Bashour A, Gonzalez-Stawinski GV. Have changes in ECMO technology impacted outcomes in adult patients developing postcardiotomy cardiogenic shock? J Card Surg 2012;27:246-52

25. Mikus E, Tripodi A, Calvi S, Del Giglio M, Cavallucci A, Lamarra M. CentriMag venoarterial extracorporeal membrane oxygenation supporta $s$ treatment for patients with refractory postcardiotomy cardiogenic shock. ASAIO J 2013:59:18-23

26. Slottosch I, Liakopoulos O, Kuhn E, et al. Outcomes after peripheral extracorporeal membrane oxygenation therapy for postcardiotomy cardiogenic shock: a single centre experience. J Surg Res 2013;181:47-55

27. Unosawa S, Sezai A, Hata M, et al. Long-term outcomes of patients undergoing ECMO for refractory postcardiotomy cardiogenic shock. Surg Today 2013;43:264-70

28. Ariyaratnam P, McLean LA, Cale ARJ, Loubani M. Extracorporeal membrane oxygenation for the post-cardiotomy patient. Heart Fail Rev 2014;19:717-25

29. Li CL, Wang H, Jia M, Ma N, Meng X, Hou XT. The early dynamic behaviour of lactate is linked to mortality in postcardiotomy patients with extracorporeal membrane oxygenation support: a retrospective observational study. J Thorac Cardiovasc Surg 2015;149:1445-50

30. Saxena P, Neal J, Joyce LD, et al. Extracorporeal membrane oxygenation support in postcardiotomy elderly patients: the Mayo Clinic experience. Ann Thorac Surg 2015;99:205360 
31. Khorsandi M, Shaikhrezai K, Prasad S, et al. Advanced mechanical circulatory support for post-cardiotomy cardiogenic shock: a 20 -year outcome analysis in a non-transplant unit. $J$ Cardio-Thorac Surg 2016;11:29-35

32. Dilstelmaier K, Schrutka L, Binder C, et al. Cardiac arrest does not affect survival in postoperative cardiovascular surgery patients undergoing extracorporeal membrane oxygenation. Resuscitation 2016;104:24-7

33. Mazzeffi MA, Sanchez PG, Herr D, et al. Outcomes of extracorporeal cardiopulmonary resuscitation for refractory cardiac arrest in adult cardiac surgery patients. $J$ Thorac Cardiovasc Surg 2016;152:1133-9

34. Chen SW, Tsai FC, Lin YS, et al. Long-term outcomes of extracorporeal membrane oxygenation support for postcardiotomy shock. J Thorac Cardiovasc Surg 2017;154:469-77

35. Guihaire J, Dang Van S, Rouze S, et al. Clinical outcomes in patients after extracorporeal membrane oxygenation support for post-cardiotomy cardiogenic shock: a single-centre experience. Interact Cardiovasc Thorac Surg 2017, doi: 10.1093/icvts/ivx155.

36. Raffa GM, Gelsomino S, Sluijpers N, et al. In-hospital outcome of post-cardiotomy extracorporeal life support in adult patients: the 2007-2017 Maastricht experience. Crit Care Resusc. 2017;19:53-61.

37. Fux, T, Holm M, Corbascio M, H. Lund LH, van der Linden J. Venoarterial Extracorporeal Membrane Oxygenation for Postcardiotomy Shock: Risk Factors for Mortality. J Thorac Cardiovasc Surg 2018;156:1894-1902.e3, doi.org/10.1016/j.jtcvs.2018.05.061.

38. Ranucci M, Ballotta A, Kandil H, et al. Bivalirudin-based versus heparin anticoagulation for postcardiotomy extracorporeal membrane oxygenation. Critical Care 2011;15:275-85

39. Ranucci M, Barushnikova E, Isgrò G, et al. Heparin-like effect in post-cardiotomy extracorporeal membrane oxygenation patients. Critical Care 2014;18:504-10

40. Smith C, Bellomo R, Raman JS, et al. An extracorporeal membrane oxygenation-based approach to cardiogenic shock in an older population. Ann Thorac Surg 2001;71:1421-7 
41. Saito S, Nakatani T, Kobayashi J, et al. Is extracorporeal life support contraindicated in elderly patients? Ann Thorac Surg 2007;83:140-5

42. Lan C, Tsai PR, Chen YS, Ko WJ. Prognostic factors for adult patients receiving extracorporeal membrane oxygenation as mechanical circulatory support - A 14-year experience at a medical center. Artif Organs 2010;34:59-64

43. Hei F, Lou S, Li J, et al. Five-year results of 121 consecutive patients treated with extracorporeal membrane oxygenation at Fu Wai hospital. Artif Organs 2011;35:572-8

44. Raffa GM, Kowalewski M, Brodie D, et al. Meta-Analysis of Peripheral or Central ECMO in Postcardiotomy and Non-Postcardiotomy Shock. Ann Thorac Surg. 2018, doi: 10.1016/j.athoracsur.2018.05.063.

45. LoforteA, Marinelli G, Musumeci F, et al. Extracorporeal membrane oxygenation support in refractory cardiogenic shock: treatment strategies and analysis of risk factors. Artif Organs 2014;38:129-41

46. Saed D, Stosik H, Islamovic M, et al. Femoro-femoral versus atrio-aortic ECMO: selecting the ideal cannulation technique. Artif Organ 2014;38:549-55

47. Carroll BJ, Shah RV, Murthy V, et al. Clinical features and outcomes in adults with cardiogenic shock supported with extracorporeal membrane oxygenation. Am J Cardiol 2015;116:1624-30

48. Truby L, Mundy L, Kalesan B, et al. Contemporary outcomes of venoarterial extracorporeal membrane oxygenation for refractory cardiogenic shock at a large tertiary care center. ASAIO $J$ 2015;61:403-9

49. Brunet J, Valette X, Ivascau C, et al. Extracorporeal life support for refractory cardiac arrest or shock: a 10-year study. ASAIO J 2015;61:676-81

50. Tarzia V, Bertolussi G, Bianco R, et al. Extracorporeal life support in cardiogenic shock: impacts of acute versus chronic etiology on outcome. J Thorac Cardiovasc Surg $2015 ; 150: 333-40$ 
51. Narotsky DL, Mosca MS, Mochari-Greenberger H, et al. Short-term and long-term survival of veno-arterial extracorporeal membrane oxygenation in an adult patient population: does older age matter? Perfusion 2016;31:366-75

52. Aso S, Matsui H, Fushimi K, Yasunaga H. In-hospital mortality and successful weaning from veno-arterial extracorporeal membrane oxygenation: analysis of 5,263 patients using a national inpatient database in Japan. Critical Care 2016;20-80

53. Demodion P, Fournel L, Golmard JL, Niculescu M, Pavie A, Leprince P. Predictors of 30day mortality and outcome in cases of myocardial infarction with cardiogenic shock treated by extracorporeal membrane oxygenation. Eur J Cardio-Thorac Surg 2014;45:47-54

54. Takayama H, Truby L, Koekort M, et al. Clinical outcome of mechanical circulatory support for refractory cardiogenic shock in the current era. J Heart Lung Transplant 2013;32:106-11

55. Xie A, Phan K, Tsai YC, Yan TD, Forrest P. venoarterial extracorporeal membrane oxygenation for cardiogenic shock and cardiac arrest: a metanalysis. J Cardio-Thorac Vasc Anesth 2015;29:637-45

56. Nakamura H, Yamaguchi H, Amano A, Nakao T. Veno-venous extracorporeal membrane oxygenation is effective against post-cardiotomy acute respiratory failure in adults. Gen Thorac Cardiovasc Surg 2013;61:402-8

57. Song JH, Woo WK, Song SH, et al. Outcome of veno-venous extracorporeal membrane oxygenation use in acute respiratory distress syndrome after cardiac surgery with cardiopulmonary bypass. J Thorac Dis 2016;8:1804-13

58. Chou NK, Chi NH, Wu IW, et al. Extracorporeal membrane oxygenation to rescue cardiopulmonary failure after heart transplantation: a single-centre experience. Transplant Proceed 2010;42:943-5

59. Borges Lima E, da Cunha CR, Barzilai VS, et al. Experience of ECMO in primary graft dysfunction after orthotopic heart transplantation. Arq Bras Cardiol 2015;105:285-91 
60. D’Alessandro C, Aubert S, Golmard JL, et al. ECMO temporary support for early graft failure after cardiac transplantation. Eur J Cardio-Thorac Surg 2010;37:343-9

61. Kittleson MM, Patel J, Moriguchi J, et al. Heart transplant recipients supported with ECMO: outcomes from a single-centre experience. J Heart Lung Transplant 2011;30:1250-6

62. Listijono D, Watson A, Pye R, et al. Usefulness of ECMO for early cardiac allograft dysfunction. J Heart Lung Tranplant 2011;30:783-9

63. Dandel M, Krabatsch T, Valk F. Left ventricular versus biventricular mechanical support: decision making and strategies for avoidance of right heart failure after left ventricular assist device implantation. Int J Cardiol 2015;198:241-50

64. Haneya A, Philipp A, Puehle T, et al. Temporary percutaneous right ventricular support using a centrifugal pump in patients with postoperative acute refractory right ventricular failure a er le ventricular assist device implantation. Eur J Cardiothorac Surg 2012;41:219-23

65. Argiriou M, Kolokotron SM, Sakellaridis T, et al. Right heart failure post left ventricular assist device implantation. J Thorac Dis 2014;6:552-9

66. Zhao Y, Xing J, Du Z, Liu F, Hou X. Extracorporeal cardiopulmonary resuscitation for adult patients who underwent post-cardiac surgery. Eur J Med Res 2016;20:83-9

67. Society of Thoracic Surgeons Task Force on Resuscitation After Cardiac S. The Society of Thoracic Surgeons Expert Consensus for the Resuscitation of Patients Who Arrest After Cardiac Surgery. Annals Thorac Surg 2017;103:1005-20

68. Ranney DN, Benrashid E, Meza JM, et al. Central cannulation as a viable alternative to peripheral cannulation in extracorporeal membrane oxygenation. Sem Thorac Cardiovasc Surg 2017;29:188-95

69. Navia J, Atik FA, Beyer EA, Ruda Vega P. Extracorporeal membrane oxygenation with right axillary artery perfusion. Ann Thorac Surg 2005;79:2163-5

70. Biscotti M, Bacchetta M. The "Sport Model": extracorporeal membrane oxygenation using the subclavian artery. Ann Thorac Surg 2014;98:1487-9 
71. Babu A. Techniques of venoarterial ECMO support and conversion to temporary LVAD. $O p$ Tech Thorac Cardiovasc Surg 2014;19:365-79

72. Meani P, Gelsomino S, Natour E, et al. Modalities and effects of left ventricular unloading on extracorporeal life support: a review of the literature. Eur J Heart Fal 2017;19:81-88

73. Russo JJ, Aleksova N, Pitcher I, et al. Left ventricular unloading during extracorporeal membrane oxygenation in patients with cardiogenic shock. J Am Coll Cardiol 2019;73:65462

74. Pappalardo F, Schulte C, Pieri M, et al. Concomitant implantation of Impella on top of VAECMO may improves survival of patients with cardiogenic shock. Eur J Heart Fail 2017;19:404-12

75. Tepper S, Faraz Masood M, Baltazar Garcia M, et al. Left ventricular unloading by Impella device versus surgical vent during extracorporeal life support. Ann Thorac Surg $2017 ; 104: 861-7$

76. Patel SM, Lipinski J, Al-Kindi SG, et al. Simultaneous venoarterial extracorporeal membrane oxygenation and percutaneous left ventricular decompression therapy with Impella is associated with improved outcomes in refractory cardiogenic shock. ASAIO J 2019;65:21-8

77. Schrage B, Burkhoff D, Rubsamen N, et al. Unloading of the left ventricle during venoarterial extracorporeal membrane oxygenation therapy in cardiogenic shock. J Am Coll Cardiol HF $2018 ; 6: 1035-43$

78. Cheng R, Hachamovitch R, Makkar R, et al. Lack of survival benefit found with the use of intra-aortic balloon pump in ECMO: a pooled experience of 1517 patients. J Invas Cardiol $2015 ; 27: 453-8$

79. Nuding S, Werdan K. IABP plus ECMO - Is one and one more than two? J Thorac Dis 2017;9:961-4 
80. Pappalardo F, Montisci A. Veno-arterial extracorporeal membrane oxygenation (VA-ECMO) in post-cardiotomy cardiogenic shock: how much pump flow is enough? J Thorac Dis 2016;8:1444-8

81. Haft JW. Temporary mechanical circulatory support for post-cardiotomy shock: don't come late to the party. $J$ Thorac Cardiovasc Surg 2015;149:1451-2

82. Dilstelmaier K, Roth C, Schrutka L, et al. Beneficial effects of levosimendan on survival in patients undergoing extracorporeal membrane oxygenation after cardiovascular surgery. Brit J Anesth 2016;117:52-8

83. Affronti A, di Bella I, Carino D, Ragni T. Levosimendan may improve weaning outcomes in venoarterial ECMO patients. ASAIO J. 2013;59:554-7

84. Distelmaier K, Wiedemann D, Binder C, et al. Duration of extracorporeal membrane oxygenation support and survival in cardiovascular surgery patients. $\mathrm{J}$ Thorac Cardiovasc Surg 2018;155;2471-6

85. Cheng R, Hachamovitch R, Kittleson M, et al. Complications of extracorporeal membrane oxygenation for treatment of cardiogenic shock and cardiac arrest: a meta-analysis of 1,866 adult patients. Ann Thorac Surg 2014;97:610-6

86. Lorusso R, Barili F, Di Mauro M, et al. In-hospital neurologic complications in adult patients undergoing venoarterial extracorporeal membrane oxygenation: Results from the Extracorporeal Life Support Organization Registry. Crit Care Med 2016;44:e964-e972

87. Lorusso R, Gelsomino S, Parise O, et al. Neurologic injury in adults supported with venovenous ECMO for respiratory failure: findings from the Extracorporeal Life Support Organization Registry. Crit Care Med 2017;45:1389-97

88. Wong JK, Smith TN, Pitcher HT, Hirose H, Cavarocchi NC. Cerebral and lower limb near infra-red spectroscopy in adults on extracorporeal membrane oxygenation. Artif Organs. 2012;36:659-67

89. Lorusso R, Gelsomino S, Parise O, et al. Veno-Arterial Extracorporeal Membrane 
Oxygenation for Refractory Cardiogenic Shock in Elderly Patients: Trends in Application and Outcome from the Extracorporeal Life Support Organization (ELSO) Registry. Ann Thorac Surg 2017;104:62-9

90. Rastan AJ, Lachmann N, Walther T, et al. Autopsy findings in patients on postcardiotomy ECMO. Int J Artif Organs 2006;29:1121-31

91. Marasco SF, Vale M, Pellegrino V, et al. Extracorporeal membrane oxygenation in primary graft failure after heart transplant. Ann Thorac Surg 2010;90:1541-7

92. Takeda K, Li B, Garan AR, et al. Improved outcome from ECMO versus ventricular assist device temporay support of primary graft dysfunction after heart transplant. Heart Lung 2017;36:640-6

93. Rubino A, Costanzo D, Stanszus D, et al. Central veno-arterial extracorporeal membrane oxygenation (C-VA-ECMO) after cardio-thoracic surgery: a single-center experience. J Cardio-Thoraxc Vasc Anesth 2018;32:1169-74

94. Chen K, Hou J, Tang H, Hu S. Concurrent implantation of intra-aortic balloon pump and extracorporeal membrane oxygenation improved survival of patients with postcardiotomy shock. Artif Organs 2019;43:142-9

95. Yan X, Jia S, Meng X, et al. Acute kidney injury in adult postcardiotomy patients with extracorporeal membrane oxygenation: evaluation of the RIFLE classification and the Acute Kidney Injury Network criteria. Eur J Cardio-Thorac Surg 2010;37:334-8

96. Roth C, Schrutka L, Binder C, et al. Liver function predicts survival in patients undergoing extracorporeal membrane oxygenation following cardiovascular surgery. Crit Care $2016 ; 20: 57$

97. Dilstelmaier K, Winter MP, Rutzler K, et al. Serum butyrylcholiesterase predicts survival after extracorporeal membrane oxygenation after cardiovascular surgery. Crit Care 2014;18:R24

98. Rahmanian PB, Adams DH, Castillo JG, Carpentier A, Filsoufi F. Predicting hospital mortality and analysis of long-term survival after major non-cardiac complications in cardiac 
surgery patients. Ann Thorac Surg 2010;90:1221-9

99. Stephens RS, Shah AS, Whitman GJR. Lung injury and acute respiratory distress syndrome after cardiac surgery. Ann Thorac Surg 2013;95:1122-9

100. Peek G, Mugford M, Tiruvoipati R, et al. Efficacy and economic assessment of conventional ventilator support versus extracorporeal membrane oxygenation for severe adult respiratory failure (CESAR): a multicentre randomised controlled trial. Lancet 2009;374:1351-63

101. Schmidt M, Bailey M, Sheldrake J, et al. Predicting survival after ECMO for severe acute respiratory failure. Am J Respir Crit Care Med 2014;189:1374-8

102. Lin TW, Tsai MT, Hu YN, et al. Postoperative ECMO support for acute type A aortic dissection. Ann Thorac Surg 2017;104:827-33

103. Rao V. Defying death: can new ECMO technology improve the outcome of postcardiotomy shock? J Card Surg 2012;27:253-4

104. Arora RC, Wischmeyer PE, Singal RK. Extracorporeal membrane oxygenation postcardiotomy: "With great power come great responsibility". J Thorac Cardiovasc Surg $2017 ; 153: 102-3$

105. Burkhart HM, Riley JB, Lynch JJ, et al. Simulation-based postcardiotomy extracorporeal membrane oxygenation crisis training for thoracic surgery residents. Ann Thorac Surg 2013;95:901-6 


\title{
CHAPTER 7
}

Structured Review of Post-Cardiotomy Extracorporeal Membrane Oxygenation:

\author{
Part 2 - Pediatric Patients
}

Roberto Lorusso*, Giuseppe Maria Raffa*, Mariusz Kowalewski, Khalid Alenizy, Niels Sluijpers, Maged Makhoul, Daniel Brodie, Mike McMullan, I-Wen Wang, Paolo Meani, Graeme MacLaren, Heidi Dalton, Ryan Barbaro, Xiaotong Hou, Nicholas Cavarocchi, Yih-Sharng Chen, Ravi Thiagarajan, Peta Alexander, Bahaaldin Alsoufi, Christian A Bermudez, Ashish S Shah, Jonathan Haft, Lilia Oreto, David A D'Alessandro, Udo Boeken, Glenn Whitman

*Equally contributor

J Heart Lung Transplant. 2019 Nov;38(11):1144-1161 


\begin{abstract}
Veno-arterial extracorporeal membrane oxygenation (V-A ECMO) is established therapy for shortterm circulatory support for children with life-treating cardiorespiratory dysfunction. In children with congenital heart disease (CHD), ECMO is commonly used to support patients with post-cardiotomy shock or complications including intractable arrhythmias, cardiac arrest, and acute respiratory failure. Cannulation configurations include central, when the right atrium and aorta are utilized in patients with recent sternotomy, or peripheral, when cannulation of the neck or femoral vessels are used in nonoperative patients. ECMO can be used to support any form of cardiac disease including univentricular palliated circulation. Although V-A ECMO is commonly used to support children with CHD, veno-venous ECMO (V-V ECMO) has been used in selected patients with hypoxemia or ventilatory failure in the presence of good cardiac function. ECMO use and outcomes in the CHD population are mainly informed by single-center studies and reports from collated registry data. Significant knowledge gaps remain, including optimal patient selection, timing of ECMO deployment, duration of support, anticoagulation, complications, and the impact of these factors on short- and long-term outcomes. This report, therefore, aims to present a comprehensive overview of the available literature informing patient selection, ECMO management, and in-hospital and early post-discharge outcomes in pediatric patients treated with ECMO for post-cardiotomy cardiorespiratory failure.
\end{abstract}




\section{Introduction}

Mechanical circulatory support (MCS) is well-established therapy for children with severe refractory pulmonary or cardiac failure. ${ }^{1}$ In the 1970 s, the first use of extracorporeal circulatory support in infants with congenital heart disease (CHD) was reported, followed by a longer extracorporeal membrane oxygenation (ECMO) run after surgical correction of Tetralogy of Fallot (TOF). ${ }^{2,3}$ Despite the availability of other modes of support, including ventricular assist devices, ECMO remains the most commonly used form of MCS in the pediatric population. ${ }^{4}$ In January 2019 the Extracorporeal Life Support Organization (ELSO) Registry reported 19,629 cardiac ECMO cases in neonates and children from 350 international centers between 1990 and 2019 (https://www.elso.org/Registry/Statistics/International Summary.aspx). According to the ELSO registry, hypoplastic left heart syndrome (HLHS) was the most common CHD diagnosis for neonates supported with ECMO, and cyanotic CHD with decreased pulmonary flow (e.g. TOF, double outlet right ventricle, and Ebstein's anomaly of the tricuspid valve) were the most common CHD diagnoses associated with cardiac ECMO in children.

Veno-Arterial ECMO (V-A ECMO) is utilized in children with cardiac failure after CHD surgery, in order to augment cardiac output and facilitate respiratory gas exchange. Indications for V-A ECMO in this population include failure to wean from cardiopulmonary bypass (CPB), thrombosis of systemic-to-pulmonary artery shunts in patients with palliated single ventricle circulation, intractable arrhythmias, postoperative low cardiac output syndrome, and cardiac arrest. ${ }^{1}$ Post-cardiotomy ECMO (PC-ECMO) may additionally bridge patients to myocardial recovery, or provide temporary MCS support as a bridge to cardiac transplantation or durable MCS. ${ }^{8,9}, 10$ V-A ECMO has also been described as bridge to CHD surgery in the setting of profound cyanosis, cardiogenic shock or pre-operative cardiopulmonary arrest (CPA). ${ }^{5,6,7}$

The use of ECMO to support children following CHD surgery has increased steadily during the past 3 decades. ${ }^{11-15}$ This increased use reflects growing experience with repair or palliation of complex forms of CHD, readily available ECMO equipment, point of care ECMO deployment, and the accumulated experience of ECMO management. Furthermore, advances in ECMO pump and oxygenator design, reduction of bloodprosthetic surface interaction with coated ECMO circuit tubing, and improved anticoagulation protocols have resulted in increased ECMO use. ${ }^{16}$ Despite increasing experience and improved ECMO technology, mortality 
in pediatric patients requiring ECMO support following CHD surgery is high and has remained unchanged over the last several decades. ${ }^{12}$

We aimed to summarize the current literature regarding PC-ECMO in pediatric patients with CHD. We provide a detailed and comprehensive summary of patient characteristics, ECMO management and complications, and short- and long-term outcomes of these patients. Future perspectives including novel indications, targets for clinical education, ethical considerations and optimal resource use will be highlighted.

\section{Characteristics of PC-ECMO}

\section{Trends in ECMO use}

Utilization of PC-ECMO is variable among institutions performing surgery for CHD. Differences in ECMO utilization may reflect variation in technical performance, chosen operative interventions as well as ECMO availability, local indications for use, and the cost of ECMO. Prior to 1990, several authors reported that $1.5-13 \%$ of children who underwent cardiac surgery for CHD were supported with ECMO (Table 1). ${ }^{13 \text {, }}$ ${ }^{17-19}$ Using data from the Pediatric Health Information System, which contains administrative data from 42 children's hospitals in the United States, Bratton and colleagues reported that from 2003-2014, $0.5 \%$ to $6 \%$ of children who underwent cardiac surgery for CHD were supported with ECMO.${ }^{20}$ A recent analysis using the Society of Thoracic Surgeons (STS) Congenital Heart Surgery Database identified 2287 children (2.4\%) supported postoperatively with MCS from the 96,596 operations performed for CHD from 2000-2010. ${ }^{21}$ Most were supported with ECMO (>95\%). The report showed ECMO was most commonly used in children undergoing the Norwood single ventricle palliation operation for HLHS (17\%) or complex biventricular repairs $(14 \%)$. The findings illustrated the wide variability in ECMO utilization across the CHD centers reporting to the STS database.

\section{Patient characteristics}

ECMO has been successfully deployed to support children of all ages, from newborn to adult-sized patients with CHD requiring cardiac surgery. ${ }^{16,18,22-24}$ Similarly, PC-ECMO support has been utilized in children of all sizes, although the small vessels in premature and low birthweight infants can make the placement of appropriately sized ECMO cannula challenging. PC-ECMO has been used to rescue children 
after surgery for all forms of $\mathrm{CHD}$, although it is more frequently used to support children undergoing more complex procedures (Table 1). ${ }^{23,25-27}$

\section{Indications for ECMO}

The indications for and rates of ECMO implantation in pediatric patients vary among different studies; however, common indications include failure to wean from CPB, cardiac arrest, low cardiac output syndrome or respiratory failure (Table 2). Klein and colleagues reported pathophysiology resulting in ECMO support included biventricular failure (36\%), right ventricular failure (14\%), left ventricular failure (33\%), and pulmonary hypertensive crisis $(17 \%) \cdot{ }^{17}$ Some populations are particularly high risk, for example, in a study of single ventricle patients palliated with systemic-to-pulmonary artery shunts, nearly half of 27 patients required ECMO implantation. ${ }^{28}$ And other studies appear to represent different population, for example, in two studies of PC-ECMO in children, low cardiac output as an indication for ECMO was present in $17 \%$ of 93 patients, and $92 \%$ of 73 patients. ${ }^{29,}{ }^{23}$ Cardiac or cardiopulmonary arrest occurred in $6 \%$, or $28 \%$ of the respective populations. Pulmonary arterial hypertension, arrythmia and failure to wean from CPB are also represented in different proportions (Table 2).

\section{Cannulation for ECMO}

ECMO cannulation strategy is determined by underlying the anatomy and physiology of CHD. An analysis of all pediatric patients (0-18 years old) reported to the ELSO Registry demonstrated carotid cannulation in $64 \%$ of patients, aortic cannulation in $32 \%$, and femoral cannulation in only $4 \% .{ }^{30}$ In many centers, central cannulation of the right atrium for venous drainage and aorta for arterial return is commonly used in the presence of a recent sternotomy (Figures 1-3). In many circumstances, peripheral vessel cannulation may be the preferred approach to reduce the risk of major bleeding and infections ${ }^{1}$. Vascular access for peripheral V-A ECMO cannulation can be achieved through the neck vessels (internal jugular vein and carotid artery) or through the femoral vessels (femoral vein and artery) in children weighing $>15 \mathrm{~kg}$. Children with single ventricle circulation palliated with cavopulmonary connections (bidirectional Glenn and Fontan circulations) frequently need multisite cannulation for venous drainage. In rare instance when patients have adequate cardiac function and only require lung support, veno-venous ECMO (V-V ECMO) can be used, and the cannulas for drainage and return are both placed in the venous circulation. 


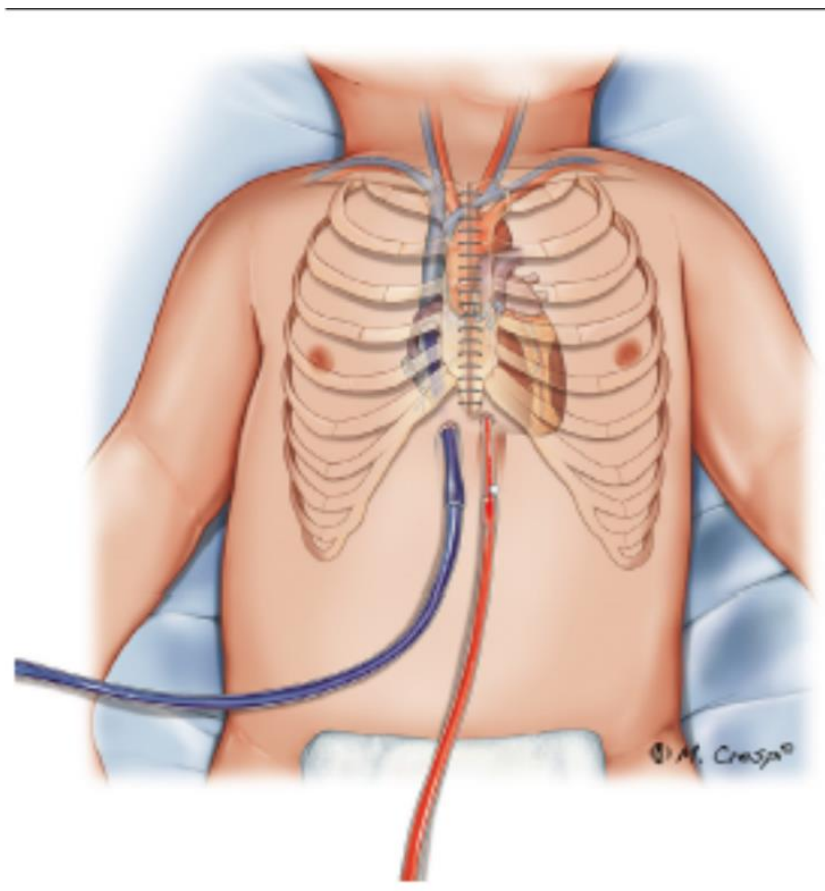

Figure 1 Post-cardiotomy extracorporeal membrane oxygenation approaches forcannulation: central cannulation (right atrium and ascending aorta cannulation) with subxyphoid exit port for cannulas, and the stemum closed.

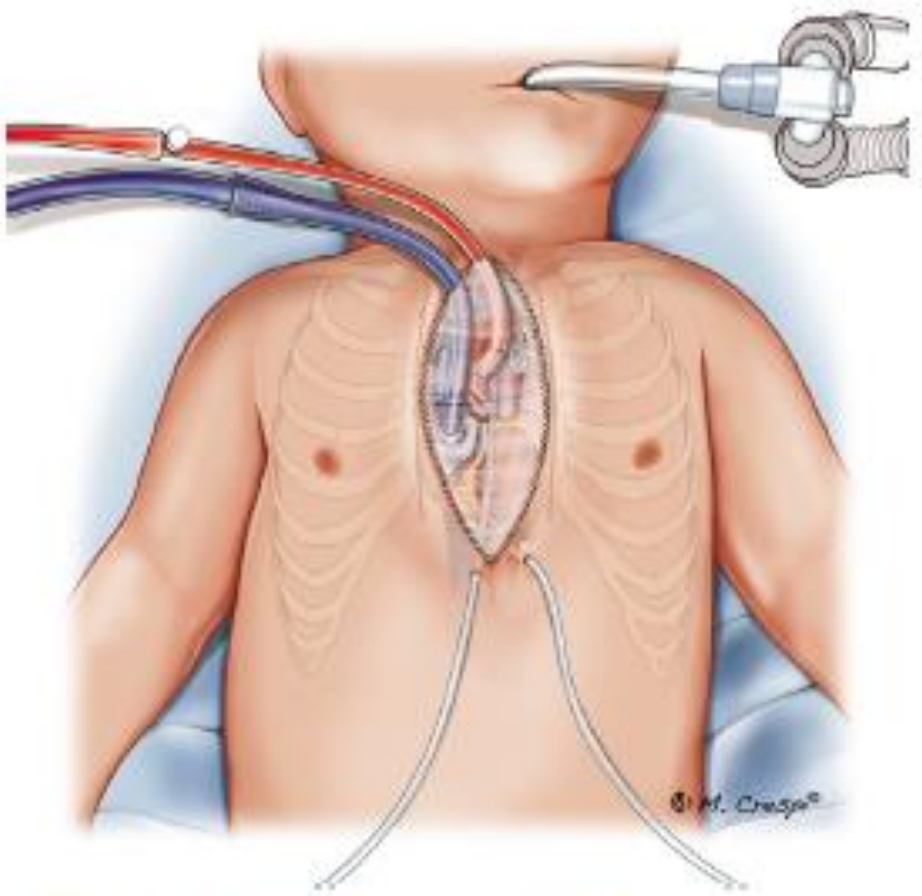

Figure 3 Post-cardiotomy extracorporeal membrane oxygenation approaches for cannulation: central cannulation with the stemum opened. 


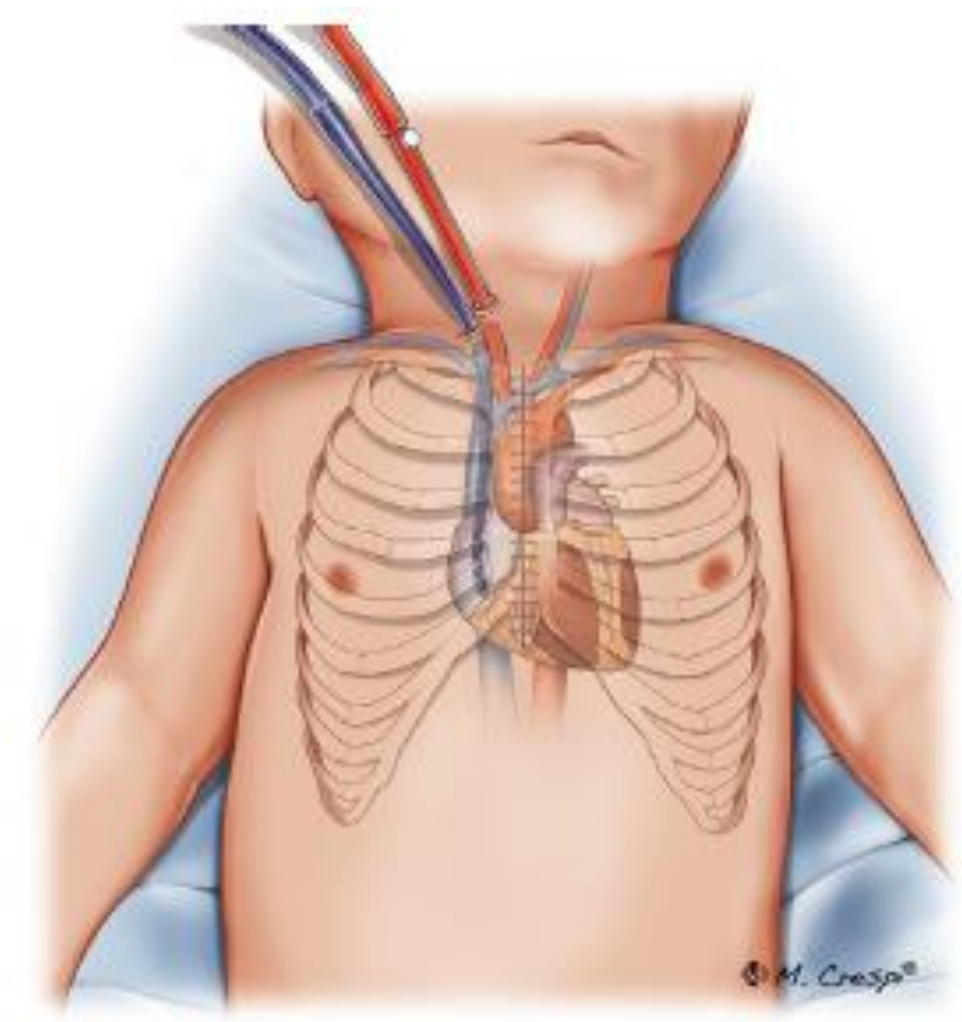

Figure 2 Post-cardiotomy extracorporeal membrane oxy genation approaches forcannulation: central cannulation (right atrium and ascending aorta cannulation) with a jugular exit port for cannulas, and the stemum closed.

Children with complex congenital heart defects are at risk for occlusion of peripheral vessels used for ECMO cannulation, because the vessels may have been accessed previously for cardiac catheterization. Thus, knowledge of vessel patency is important in children with complex CHD and previous history of multiple cardiac catheterization procedures. Chan and colleagues, in a report of 492 children with CHD supported with ECMO following cardiac arrest, showed that the use of the right carotid artery for V-A ECMO was associated with improved survival to hospital discharge as compared with transthoracic cannulation. The authors speculate that the reason for improved survival may be related to fewer cardiopulmonary resuscitation (CPR) interruptions during neck cannulation. ${ }^{31}$ Finally, the American Heart Association recently published a statement on cardiopulmonary resuscitation in children with CHD, which includes a table of suggested cannulation sites for ECMO based on the underlying circulation. ${ }^{32}$ 


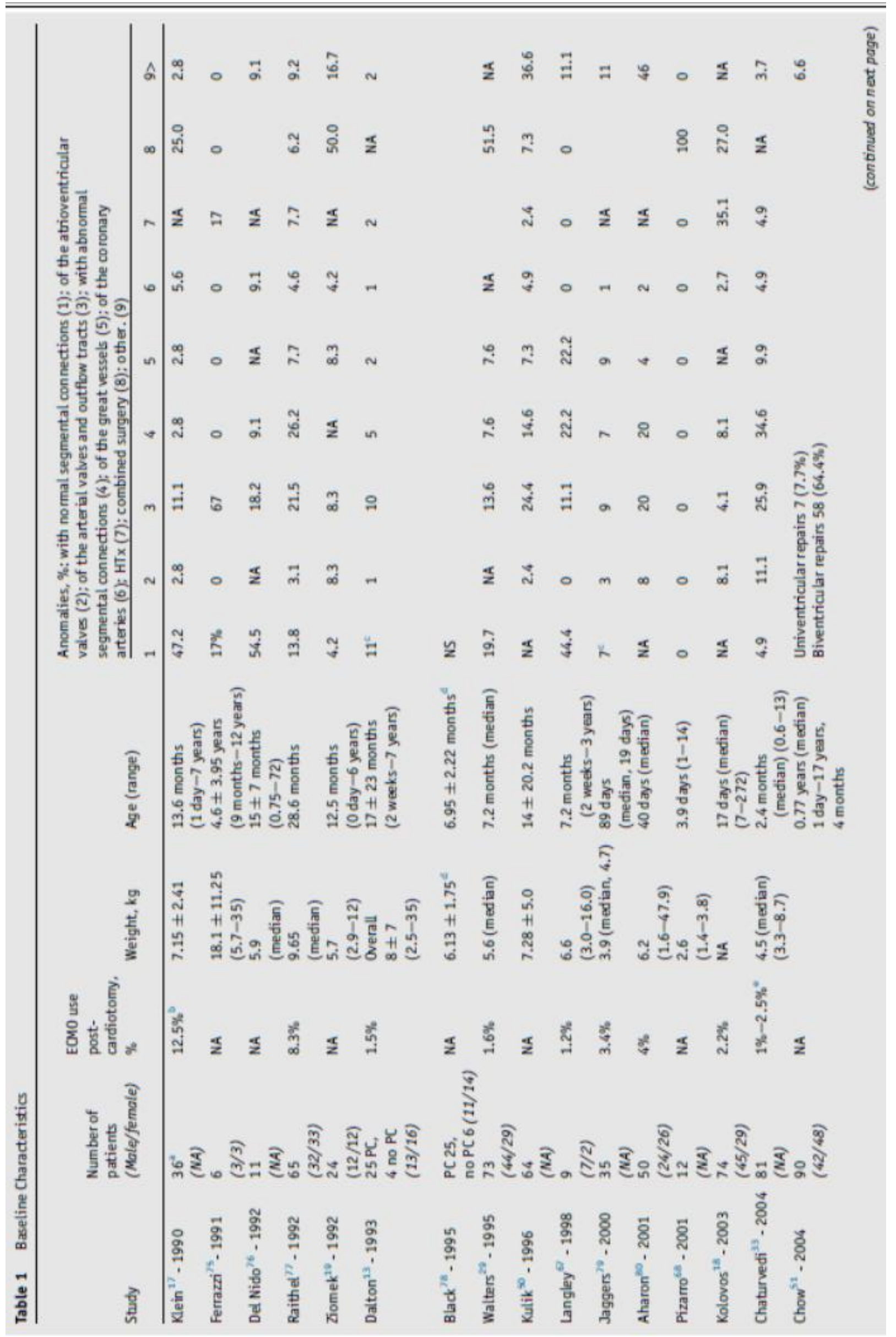




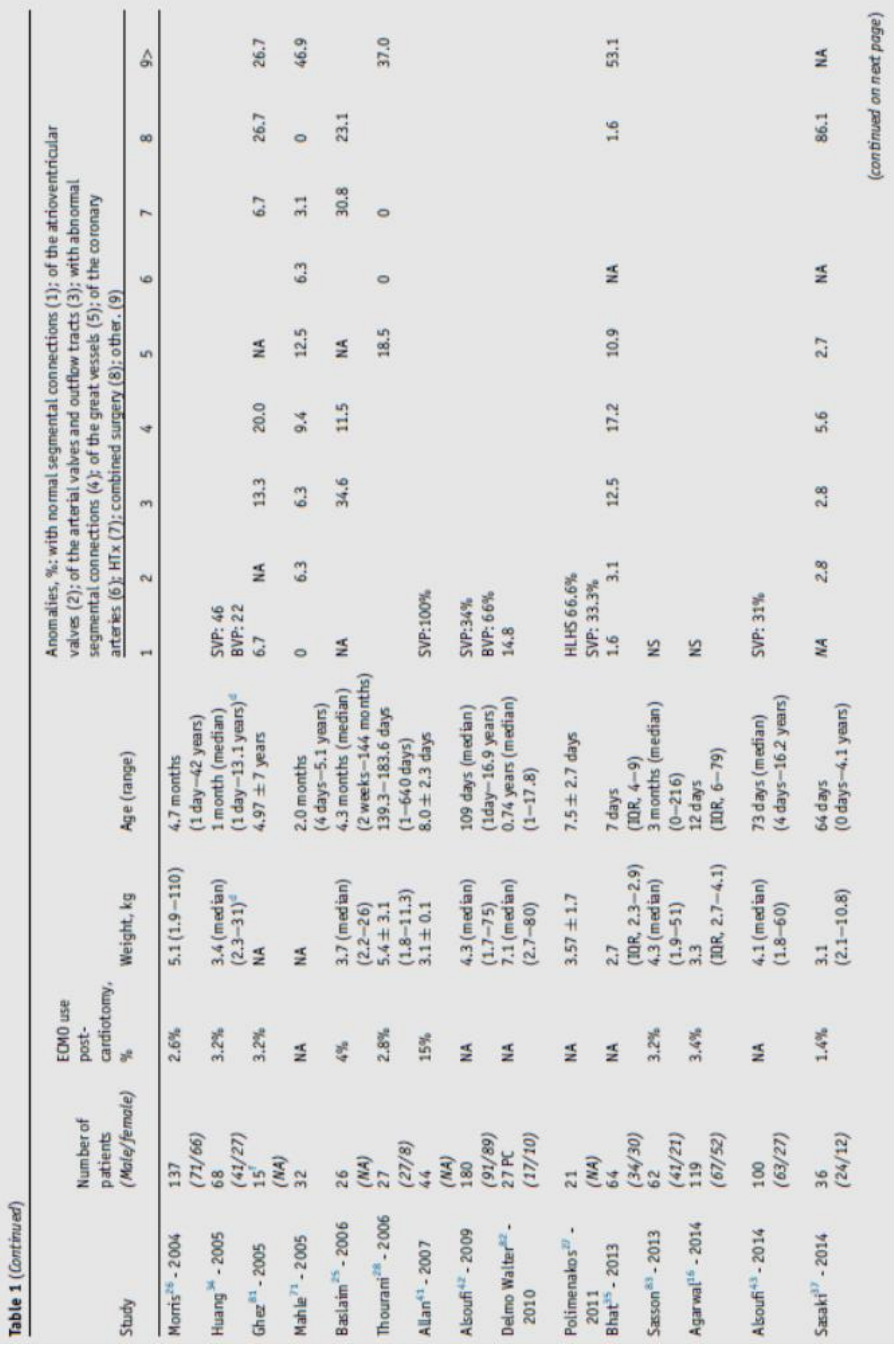




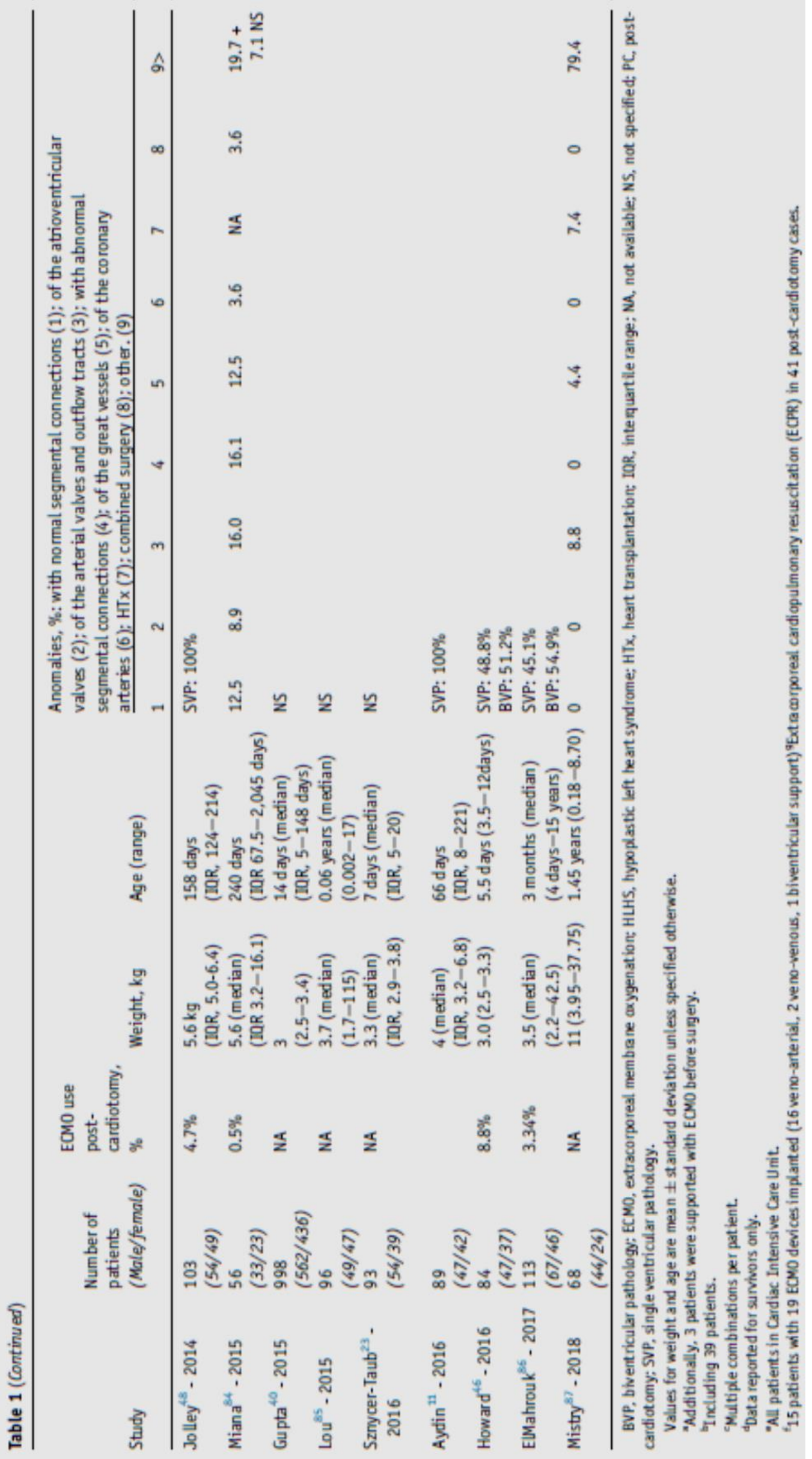


Table 2 EOMO Implant Indication, Implant Location, and Implant Access

\begin{tabular}{|c|c|c|c|c|}
\hline Study & $\begin{array}{l}\text { Indications for ECMO } \\
\text { implant, } \mathrm{n}(\%)\end{array}$ & $\begin{array}{l}\text { EOMO implant } \\
\text { location, } \mathrm{n}(\%)\end{array}$ & $\begin{array}{l}\text { ECMO implant } \\
\text { access, } \mathrm{n}(\%)\end{array}$ & $\begin{array}{l}\text { EOM0 venting, } \\
\mathrm{n}(\%)\end{array}$ \\
\hline Klein $^{17}-1990$ & $\begin{array}{l}\text { BVF } 13(36 \%) \\
\text { RVF } 5(14 \%) \\
\text { LVF } 12(33 \%) \\
\text { PVRC } 6(17 \%)\end{array}$ & $\begin{array}{l}\text { OR } 9(25 \%) \\
\text { ICU } 27(75 \%)\end{array}$ & $\begin{array}{l}\text { Neck } 30(83 \%) \\
\text { Chest } 6(17 \%)\end{array}$ & NA \\
\hline Ferrazzi ${ }^{75}-1991$ & $\begin{array}{l}\text { LVF } 1(16.6 \%) \\
\text { RVF } 4(66.6 \%) \\
\text { BVF } 1(16.6)\end{array}$ & $\begin{array}{l}\text { OR } 2(33 \%) \\
\text { ICU } 4(66 \%)\end{array}$ & Chest $6(100 \%)$ & NA \\
\hline Del Nido ${ }^{76}-1992$ & $\begin{array}{l}\text { Cardiac arrest } 9(82 \%) \\
\text { Not specified } 2(18 \%)\end{array}$ & NA & $\begin{array}{l}\text { Neck } 1(9 \%) \\
\text { Chest } 10(91 \%)\end{array}$ & $3^{2}(27 \%)$ \\
\hline Raithel $^{n}-1992$ & $\begin{array}{l}\text { Failure to wean from CPB } 20(31 \%) \\
\text { Cardiac failure } 45(69 \%)\end{array}$ & $\begin{array}{l}\text { OR } 22(34 \%) \\
\text { ICU } 43(66 \%)\end{array}$ & $\begin{array}{l}\text { Chest } 59(91 \%) \\
\text { Femoral } 6(9 \%)\end{array}$ & $\begin{array}{l}\text { LA vent } 11(17 \%) \\
\text { LV vent } 4(6 \%)\end{array}$ \\
\hline Ziomek ${ }^{10}-1992$ & $\begin{array}{l}\text { Ventricular failure } 17(71 \%) \\
\text { Pulmonary hypertension } 6(25 \%) \\
\text { Hypoxemia } 1(4 \%)\end{array}$ & $\begin{array}{l}\text { OR } 17(71 \%) \\
\text { ICU } 7(29 \%)\end{array}$ & $\begin{array}{l}\text { Neck } 9(38 \%) \\
\text { Chest } 15(62 \%)\end{array}$ & $\begin{array}{l}\text { Pulmonary artery } \\
\text { vent } 1(4 \%)\end{array}$ \\
\hline Dalton ${ }^{13}-1993$ & $\begin{array}{l}\text { BVF }+ \text { arrest } 4(15 \%) \\
\text { BVF } 8(30 \%) \\
\text { RVF } 3(11 \%) \\
\text { LVF } 2(7 \%) \\
\text { Cardiac arrest } 9(33 \%) \\
\text { Arrhythmia } 1(4 \%)\end{array}$ & NA & $\begin{array}{l}\text { Neck } 5(19 \%) \\
\text { Chest } 22(81 \%)\end{array}$ & LA vent $2^{b}(7 \%)$ \\
\hline Black $^{78}-1995$ & $\begin{array}{l}\text { Myocardial failure } 21(84 \%) \\
\text { Respiratory failure } 3(12 \%) \\
\text { Cardiac arrest } 1(4 \%)\end{array}$ & NA & $\begin{array}{l}\text { Chest } 28(90 \%) \\
\text { Femoral } 3(10 \%)^{c}\end{array}$ & NA \\
\hline Walters $^{20}-1995$ & $\begin{array}{l}\text { Arrhythmia } 1 \text { ( } 1.5 \%) \\
\text { Cardiac arrest } 4(6.1 \%) \\
\text { Low cardiac output } 61 \text { ( } 92.4 \%) \\
\text { No spontaneous electrical activity } \\
1 \text { (1.5\%) } \\
\text { Pulmonary artery hypertension } \\
14(21.2 \%)\end{array}$ & NA & $\begin{array}{l}\text { Neck } 48(73 \%) \\
\text { Chest } 18(27 \%)\end{array}$ & NA \\
\hline Kulik $^{50}-1996$ & $\begin{array}{l}\text { Ventricular dysfunction } 26(41 \%) \\
\text { Pulmonary failure } 13(20 \%) \\
\text { Pulmonary hypertension } 7(11 \%) \\
\text { Combination } 10(16 \%) \\
\text { Cause of hemodynamic instability } \\
\text { unknown } 8 \text { (13\%) }\end{array}$ & NA & $\begin{array}{l}\text { Neck } 37(58 \%) \\
\text { Chest } 20(31 \%) \\
\text { Femoral } 2(3 \%) \\
\text { Multiple } 5(8 \%)\end{array}$ & LA vent (19\%) \\
\hline Langley ${ }^{67}-1998$ & $\begin{array}{l}\operatorname{LVF}(33 \%) \\
\operatorname{RVF}(11 \%) \\
\text { BVF }(44 \%) \\
\text { Cardiac arrest }(11 \%)\end{array}$ & $\begin{array}{l}\text { OR7 }(78 \%) \\
\text { ICU } 2(22 \%)\end{array}$ & Chest $9(100 \%)$ & $0 \%$ \\
\hline Jaggers $^{\prime \prime}-2000$ & $\begin{array}{l}\text { Low cardiac output } 17 \text { ( } 49 \%) \\
\text { Failure to wean from CPB } 10(29 \%) \\
\text { Cardiac arrest } 5(14 \%) \\
\text { Arrhythmia } 3(9 \%) \\
\text { Pulmonary Hypertension } 2(6 \%) \\
\text { Hypoxia } 1 \text { ( } 3 \%) \\
\text { Biventricular dysfunction } 1(3 \%)\end{array}$ & $\begin{array}{l}\text { OR } 15(43 \%) \\
\text { ICU } 20(57 \%)\end{array}$ & NA & LA vent 1 (3\%) \\
\hline Aharon $^{80}-2001$ & $\begin{array}{l}\text { Failure to wean from CPB } 22(44 \%) \\
\text { Low cardiac output } 11(22 \%) \\
\text { Pulmonary hypertension } 7(14 \%) \\
\text { Cardiac arrest } 10(20 \%)\end{array}$ & $\begin{array}{l}\text { OR } 23(46 \%) \\
\text { ICU } 27(54 \%)\end{array}$ & $\begin{array}{l}\text { Neck } 1(2 \%) \\
\text { Chest } 49(98 \%)\end{array}$ & NA \\
\hline Pizarro $^{68}-2001$ & $\begin{array}{l}\text { Low cardiac output } 6(50 \%) \\
\text { Cardiac arrest } 2(16.6 \%) \\
\text { Respiratory failure } 2(16.6 \%) \\
\text { Unbalanced pulmo nary/systemic } \\
\text { circulation } 1(8.3 \%) \\
\text { Supraventriculartachycardia } 1(8.3 \%)\end{array}$ & $\begin{array}{l}\text { OR } 9(75 \%) \\
\text { ICU } 3(25 \%)\end{array}$ & Chest $12(100 \%)$ & NA \\
\hline
\end{tabular}




\begin{tabular}{|c|c|c|c|c|}
\hline Kolovos ${ }^{28}-2003$ & $\begin{array}{l}\text { Ventricular failure } 51(69 \%) \\
\text { Respiratory failure } 9(12 \%)\end{array}$ & NA & $\begin{array}{l}\text { Neck } 26(35 \%) \\
\text { Chest } 47(64 \%)\end{array}$ & $\begin{array}{l}\text { LA vent } 12(16 \%) \\
\text { Atrial septostomy }\end{array}$ \\
\hline & $\begin{array}{l}\text { Pulmonary hypertension } 4 \text { ( } 5 \%) \\
\text { Multip le indications (not specified) } \\
6(8 \%) \\
\text { Shunt occlusion } 4 \text { (5\%) }\end{array}$ & & Groin $1(1 \%)$ & $1(1 \%)$ \\
\hline Chaturvedi ${ }^{13}$ - 2004 & $\begin{array}{l}\text { Failure to wean from CPB } \\
\text { Low cardiac output } \\
\text { No data available }\end{array}$ & $\begin{array}{l}\text { OR } 47(58 \%) \\
\text { IOJ } 34(42 \%)\end{array}$ & $\begin{array}{l}\text { Neck } 5(6 \%) \\
\text { Chest } 76(94 \%)\end{array}$ & NA \\
\hline Chow $^{51}-2004$ & $\begin{array}{l}\text { Myocarditis } 10(11 \%) \\
\text { Cardiomyopathy } 9(10 \%) \\
\text { Cong enital Heart Disease } 71(79 \%)\end{array}$ & NA & NA & NA \\
\hline Morris $^{26}-2004$ & $\begin{array}{l}\text { Cardiac arrest } \\
\text { Failure to wean from CPB } \\
\text { Low cardiac output }\end{array}$ & & & \\
\hline Huang $^{34}-2005$ & $\begin{array}{l}\text { Failure to wean from CPB } 46(67 \%) \\
\text { Low cardiac output } 11(16 \%) \\
\text { Cardiac arrest } 11(16 \%)\end{array}$ & $\begin{array}{l}\text { OR } 46(67 \%) \\
\text { IDU } 22(33 \%)\end{array}$ & $\begin{array}{l}\text { Chest } 66(97 \%) \\
\text { Femoral } 2(3 \%)\end{array}$ & LA vent $12(18 \%)$ \\
\hline Ghez -2005 & $\begin{array}{l}\text { Hemodynamic failure } 12(63 \%) \\
\text { Respiratory failure } 2(11 \%) \\
\text { Mixed failure } 5(8 \%)\end{array}$ & $\begin{array}{l}\text { OR } 4(27 \%) \\
\text { IOJ } 11(73 \%)\end{array}$ & $\begin{array}{l}\text { Neck } 4(21 \%) \\
\text { Chest } 11(58 \%) \\
\text { Femoral } 4^{4}(21 \%)\end{array}$ & NA \\
\hline Mahle $^{71}-2005$ & $\begin{array}{l}\text { Cardiopulmonary arrest } 18(56 \%) \\
\text { Failure to wean from CPB } 11(34 \%) \\
\text { Postoperative LCOS } 2(6 \%) \\
\text { Pulmonary hypertension } 1 \text { (3\%) }\end{array}$ & NA & NA & NA \\
\hline Baslaim ${ }^{25}-2006$ & $\begin{array}{l}\text { Ventricular failure } 17(65 \%) \\
\text { Respiratory failure } 6(23 \%) \\
\text { Pulmonary hypertension } 1(4 \%) \\
\text { Allergic reaction to blood products } \\
1 \text { ( } 4 \%) \\
\text { Postoperative distal pulmonary artery } \\
\text { stenting } 1 \text { ( } 4 \%)\end{array}$ & $\begin{array}{l}\text { OR (not specified) } \\
\text { IOU (not specified) }\end{array}$ & Chest $26(100 \%)$ & NA \\
\hline Thourani ${ }^{26}-2006$ & $\begin{array}{l}\text { Cardiomyopathy-myocarditis } 8 \text { (30\%) } \\
\text { Systemic-to-pulmonary artery shunt } \\
\text { dependent single ventricle } 12(44 \%) \\
\text { Postcardiotomy for biventricular repair } \\
6(22 \%) \\
\text { Arrhythmias } 1(4 \%)\end{array}$ & NA & NA & NA \\
\hline Allan ${ }^{41}-2008$ & $\begin{array}{l}\text { Myocardial failure } 22(50 \%) \\
\text { Cardiac arrest } 4(9.1 \%) \\
\text { Tamponade } 2(4.5 \%) \\
\text { Pulmonary hypertension } 1(2.3 \%) \\
\text { Respiratory failure } 3(6.8 \%) \\
\text { Shunt thrombosis/stenosis } 12(27.2 \%)\end{array}$ & NA & NS & NA \\
\hline Alsoufin $^{-2}-2009$ & $\begin{array}{l}\text { Failure to wean from CPB } 83(46 \%) \\
\text { Low cardiac output } 97(54 \%) \\
\text { Cardiac arrest } 48(27 \%)\end{array}$ & $\begin{array}{l}\text { OR } 83(46 \%) \\
\text { IQ } 92(51 \%) \\
\text { Catherization } \\
\text { laboratory } 5(3 \%)\end{array}$ & $\begin{array}{l}\text { Neck } 12(6 \%) \\
\text { Chest } 168(94 \%)\end{array}$ & NA \\
\hline $\begin{array}{l}\text { Delmo Walter }{ }^{22}-2010 \\
\text { Polimenakos }\end{array}$ & $\begin{array}{l}\text { Cardiac Arrest } 27(100 \%) \\
\text { Cardiac arrest } 17(81 \%) \\
\text { Respiratory failure fo llowed by cardiac } \\
\text { arrest } 4 \text { (19\%) }\end{array}$ & $\begin{array}{l}\text { NA } \\
\text { NA }\end{array}$ & $\begin{array}{l}\text { NA } \\
\text { NA }\end{array}$ & $\begin{array}{l}\text { LV vent } 5(19 \%) \\
\text { NA }\end{array}$ \\
\hline Bhat ${ }^{35}-2013$ & $\begin{array}{l}\text { Failure to wean from CPB } 39(61 \%) \\
\text { Low cardiac output } 9(14 \%) \\
\text { Cardiac arrest } 16(25 \%)\end{array}$ & $\begin{array}{l}\text { OR } 39(61 \%) \\
\text { IQU } 25(39 \%)\end{array}$ & Chest 59 (92\%) & NA \\
\hline Sasson $^{28}-2013$ & $\begin{array}{l}\text { Failure to wean from CPB } 53 \text { ( } 83 \%) \\
\text { Cardiac arrest } 9(17 \%)\end{array}$ & $\begin{array}{l}\text { OR } 53(83 \%) \\
\text { IDU } 9(17 \%)\end{array}$ & $\begin{array}{l}\text { Neck } 2(3 \%) \\
\text { Chest } 60(97 \%)\end{array}$ & NA \\
\hline
\end{tabular}




\begin{tabular}{|c|c|c|c|c|}
\hline Agarwal $^{16}-2014$ & $\begin{array}{l}\text { Failure to wean from CPB } 50(42 \%) \\
\text { Low cardiac output } 25(21 \%) \\
\text { Cardiac arrest } 34 \text { ( } 29 \%) \\
\text { Other (including arrhythmia, } \\
\text { respiratory failure and pulmonary } \\
\text { hypertension) } 10(8 \%)\end{array}$ & $\begin{array}{l}\text { OR } 58(49 \%) \\
\text { ICU } 61(51 \%)\end{array}$ & NA & NA \\
\hline Alsoufi ${ }^{43}-2014$ & $\begin{array}{l}\text { Failure to wean from CPB } 34 \text { (34\%) } \\
\text { Low cardiac output } 29(29 \%) \\
\text { Cardiac arrest } 37(37 \%)\end{array}$ & $\begin{array}{l}\text { OR } 34(34 \%) \\
\text { ICU } 66(66 \%)\end{array}$ & NA & NA \\
\hline Sasaki ${ }^{37}-2014$ & $\begin{array}{l}\text { Failure to wean from CPB } 14(39 \%) \\
\text { Low cardiac output } 15(42 \%) \\
\text { Other (not specified) } 7(19 \%)\end{array}$ & $\begin{array}{l}\text { OR } 18(50 \%) \\
\text { ICU } 18(50 \%)\end{array}$ & Chest $36(100 \%)$ & NA \\
\hline Jolley ${ }^{48}-2014$ & $\begin{array}{l}\text { cardiac } 90(87 \%) \\
\text { ECPR } 9,(9 \%) \\
\text { pulmonary } 4(4 \%)\end{array}$ & NA & $\begin{array}{l}\text { Chest } 89(86 \%) \\
\text { Neck } 14(103 \%)\end{array}$ & NA \\
\hline $\operatorname{Miana}^{84}-2015$ & Failure to wean from CPB $56(100 \%)$ & OR $56(100 \%)$ & Chest $56(100 \%)$ & $\begin{array}{l}\text { Inter atrial vent } \\
\text { LA vent } \\
\text { (not specified) }\end{array}$ \\
\hline $\mathrm{Gupta}^{22}-2015$ & NA & NA & NA & NA \\
\hline Louss -2015 & $\begin{array}{l}\text { Failure to wean from CPB } 24(25 \%) \\
\text { Low cardiac output } 26(27 \%) \\
\text { Cardiac arrest } 46(48 \%)\end{array}$ & NA & $\begin{array}{l}\text { Chest } 90(94 \%) \\
\text { Peripheral } \\
\text { (not specified) } \\
3(3 \%) \\
\text { Both } 3(3 \%)\end{array}$ & NA \\
\hline Szmycer-Taub ${ }^{23}$ - 2016 & $\begin{array}{l}\text { Low cardiac output } 16 \text { (17\%) } \\
\text { Failure to wean from CPB } 44(47 \%) \\
\text { Pulmonary hypertension } 2(2 \%) \\
\text { Combined cardiac and respiratory } \\
\text { failure } 26 \text { ( } 28 \%) \\
\text { Respiratory failure } 2(2 \%) \\
\text { Shunt occlusion } 3(3 \%)\end{array}$ & $\begin{array}{l}\text { OR } 42(45 \%) \\
\text { ICU } 51(55 \%)\end{array}$ & $\begin{array}{l}\text { Neck } 13(14 \%) \\
\text { Chest } 80(86 \%)\end{array}$ & NA \\
\hline Aydin ${ }^{11}-2016$ & $\begin{array}{l}\text { Respiratory failure } 59(63 \%) \\
\text { Cardiac failure } 30(32 \%)\end{array}$ & NA & $\begin{array}{l}\text { Neck } 57(64 \%) \\
\text { Chest } 24(27 \%) \\
\text { Femoral } 10(11 \%)\end{array}$ & NA \\
\hline Howard ${ }^{46}-2016$ & $\begin{array}{l}\text { Cardiac arrest } 39(46 \%) \\
\text { Failure to wean from CPB } 21(25 \%) \\
\text { LCOS } 18(21 \%) \\
\text { Hypoxemia } 6(7 \%)\end{array}$ & NA & Chest $82(98 \%)$ & NA \\
\hline ElMahrouk $^{36}-2017$ & $\begin{array}{l}\text { Ventricular dysfunction } 59(52 \%) \\
\text { Pulmonary Failure } 34(30 \%) \\
\text { Cardiac arrest } 10(9 \%) \\
\text { Pulmonary hypertension } 7(6 \%) \\
\text { Other } 3(3 \%)^{*}\end{array}$ & $\begin{array}{l}\text { OR } 88(78 \%) \\
\text { ICU } 25(22 \%)\end{array}$ & NA & NA \\
\hline Mistry $^{87}-2018$ & $\begin{array}{l}\text { Cardiomyopathy } 28(41 \%) \\
\text { Cardiorespiratory failure } 28(41 \%) \\
\text { Congenital heart disease } 9(13 \%) \\
\text { Posttransplant rejection } 5(7.4 \%)\end{array}$ & NA & NA & $16(23.5 \%)$ \\
\hline \multicolumn{5}{|c|}{ 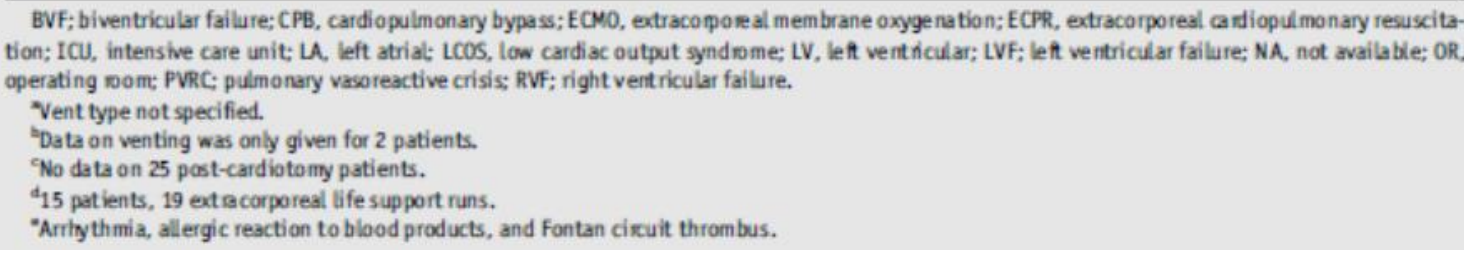 } \\
\hline
\end{tabular}

\section{PC-ECMO Outcomes}

\section{Survival and duration of ECMO support}

After PC-ECMO, survival-to-hospital-discharge ranges from 40-60\% in most studies of pediatric patients. (Table 3). Many factors influence the duration of PC-ECMO support in children, including the underlying cardiac lesion, presence of residual lesion, the family's wishes, cardiac surgical recovery time, and the applicability of bridging to transplant if recovery does not occur. Thus, the number of hours of PC-ECMO support varies greatly among different studies, ranging from 17 to more than 200 hours (Table 3$).{ }^{17,}{ }^{19}$ Survival 
to ECMO decannulation and hospital discharge also varies in the reported literature, with between 49 to $58 \%$ making it home alive. ${ }^{17,19,33}$ These mainly single center studies report higher survival than typically found in ELSO registry reports of the pediatric cardiac population. ${ }^{12}$ Longer-term survival, for example at 1 year post PC-ECMO has been reported as high as $41 \%$ (Table 3$).{ }^{33}$

Survival to hospital discharge may also vary by age and weight, with higher risk of death in neonates. 16, 18, 22-24 Infants weighing <3 kg have been reported to have high risk of death after ECMO support. ${ }^{35-37}$ In a study of 4,471pediatric patients supported with ECMO for cardiac indications reported to the ELSO registry, there were no survivors among 9 patients weighing $<1.5 \mathrm{~kg}$, and survival was $25 \%$ among those who weighed $1.5-2 \mathrm{~kg} .{ }^{36}$ In premature infants, immaturity of the choroid plexus may result in the higher incidence of intracranial hemorrhage. Birthweight $<3 \mathrm{~kg}$ has been associated with increased risk of neurological complications in PC-ECMO for CHD. ${ }^{38}$ Indeed, both prematurity and lower birthweight were associated with increased mortality and a higher incidence of neurological complications in a study of 641 neonates supported with ECMO following cardiac arrest by McMullan and colleagues. ${ }^{39}$ Bhat and colleagues also examined PCECMO used in infants weighing $3 \mathrm{~kg}$ or less; $52 \%$ of the patients were decannulated from ECMO and $28.1 \%$ survived until discharge. ${ }^{35}$ This study reported one of the longest durations of ECMO support with median 164 hours (interquartile range 95-231). (Table 3). Thus, the ability to provide adequate ECMO support is affected by size, and neurological complications may limit survival in premature neonates $(<34$ weeks gestation or birthweight $<2 \mathrm{~kg}$ ).

Survival to hospital discharge also varies widely based on the complexity of the underlying cardiac surgical procedure..$^{25,27,33,40}$ Allan and colleagues compared the indications for initiation of ECMO in infants with shunted single-ventricle physiology to the survival; $81 \%$ of patients cannulated for hypoxemia, but only $29 \%$ of those cannulated for hypotension survived to hospital discharge. ${ }^{41}$ Patients cannulated for shunt obstruction had the highest survival (83\%). In an STS Congenital Heart Surgery Database study of 2287 children supported postoperatively with MCS by Mascio and colleagues ${ }^{21}$, in-hospital mortality was highest among in patients supported after repair of truncus arteriosus, the Norwood single ventricle palliation operation for HLHS, or the Ross-Konno operation for repair of left ventricular outflow tract obstruction. In these circumstances, poor outcomes may be due to the accidental damage of the coronaries during surgery, serious aortic regurgitation after an incomplete repair, or an inherently poor systemic ventricle due to congenital aortic 
stenosis. Patients undergoing repair of an anomalous coronary artery from the left pulmonary artery had the best survival. ${ }^{21}$

Table 3 Duration of EOM0 Support, Weaning Rate, In-Hospital Survival, 1-Year Survival, and Predictors of In-Hospital Mortality

\begin{tabular}{|c|c|c|c|c|c|}
\hline Study & Duration of EEMO, in hours or days & $\begin{array}{l}\text { Weaning } \\
\mathrm{n}(\%)\end{array}$ & $\begin{array}{l}\text { In-hospital } \\
\text { survival } \\
\mathrm{n}(\%)\end{array}$ & $\begin{array}{l}\text { 1-year } \\
\text { survival } \\
\mathrm{n}(\%)\end{array}$ & Predictors of in -hospital mortality \\
\hline Klein $^{17}-1990$ & $\begin{array}{l}\text { Total: } 105 \pm 46 \text { hours } \\
\text { Survivors: } 110 \pm 27 \text { hours (mean } \pm \text { SD) }\end{array}$ & $22(61 \%)$ & $21(58 \%)$ & NA & NA \\
\hline Ferrazzi's - 1991 & $126 \pm 47$ hours (range, $67-173$ hours) & $3(50 \%)$ & $2(33 \%)$ & NA & NA \\
\hline Del Nido N-1992 & $112 \pm 18$ hours & $\begin{array}{l}7(66 \%) \\
1 \text { HTx included }\end{array}$ & $7(64 \%)$ & $6(55 \%)$ & NA \\
\hline Raithel" - 1992 & $\begin{array}{l}\text { Survivors: } 87.9 \text { hours, } 45-197.5 \text { hours } \\
\text { (mean, range) }\end{array}$ & $44(68 \%)$ & $23(35 \%)$ & NA & Duration of ECM0 support, renal failure/dialysis, sepsis \\
\hline Ziomek ${ }^{10}-1992$ & 96 hours, $17-198$ hours (mean, range) & $16(75 \%)$ & $13(54 \%)$ & NA & Sepsis \\
\hline Dalton ${ }^{13}-1993$ & $113 \pm 62$ hours (mean $\pm S D$ ) & $14(67 \%)$ & $9(43 \%)$ & $7(33 \%)$ & Longer CPB time and shorter time on ECMO \\
\hline Black's -1995 & 5.7 days (mean) & $10(40 \%)$ & $10(40 \%)$ & $10(40 \%)$ & 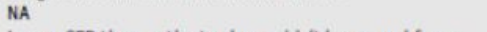 \\
\hline Walters $^{20}-1995$ & $115 \pm 6$ hours (mean $\pm S D)$ & $44(67 \%)$ & $38(58 \%)$ & NA & $\begin{array}{l}\text { Longer CPB time, patients who couldn't be weaned from } \\
\text { CPB, elevated BUN } 48 \mathrm{~h} \text { after ECMO cannulation, elevated } \\
\text { creatinine } 48 \mathrm{~h} \text { after EOMO cannulation, need of RBCs on } \\
\text { ECMO, need of plasma on ECM0, creatinine } 48 \mathrm{~h} \text { after } \\
\text { ECMO decannulation, and average right atrial pressure } 8 \mathrm{~h} \\
\text { after EOMO decannulation }\end{array}$ \\
\hline Kulik ${ }^{50}-1996$ & NA & $31(48 \%)$ & $21(33 \%)$ & NA & No significant variables \\
\hline Langley ${ }^{67}-1998$ & 121 hours $(15-648)$ & $4(44.4 \%)$ & $3(33 \%)$ & $2(22 \%)$ & Duration of ECMO \\
\hline Jaggers" -2000 & 5.6 days (median 5 days, range $0.7-16$ days) & NA & $21(61 \%)$ & $17(49 \%)$ & $\begin{array}{l}\text { Longer time on ECMO, development of renal failure, shunt } \\
\text { left open on ECMO }\end{array}$ \\
\hline Aharon ${ }^{00}-2001$ & Survivors: 89 hours (mean) (range 20-192 hours) & $30(60 \%)$ & $25(50 \%)$ & $23(45 \%)$ & $\begin{array}{l}\text { Renal failure requiring hemodialysis, EOM0 duration } \\
>72 \text { hours, pro longed (PR times ( }>45 \text { minutes) }\end{array}$ \\
\hline Pizarto $^{61}-2001$ & 67 hours (medan 48 hours) range (24-192 hours) & $12(100 \%)$ & $6(50 \%)$ & $6(50 \%)$ & ECMO initiated outside OR \\
\hline Kolovos ${ }^{18}-2003$ & 127 hours (median) (IOR $73-209$ hours) & $50(68 \%)$ & $37(50 \%)$ & NA & $\begin{array}{l}\text { CPR during ECM0 cannulation, renal failure/dialysis, } \\
\text { single ventricle physiology, lactate within } 48 \text { hours of } \\
\text { ECMO initiation }\end{array}$ \\
\hline Chaturvedi $^{13}-2004$ & 144 hours (median) (IOR 70-226 hours) & $47(58 \%)$ & $40(49 \%)$ & $33(41 \%)$ & $\begin{array}{l}\text { Circuit problems, renal failure/dialysis, residual cardiac } \\
\text { lesions, ECM0 duration, blood product transfusion, and } \\
\text { cross clamp time }\end{array}$ \\
\hline Chow $w^{51}-2004$ & 90 hours (median) 6-394 hours & NA & $41(46 \%)$ & $21(38 \%)^{*}$ & Previous CPR \\
\hline Morris $^{26}-2004$ & Survivors: 94.5 (median) (range 7 hours -15 days) & NA & $36(40 \%)$ & NA & Age $<1$ month, male sex \\
\hline Huang $^{*}-2005$ & $\begin{array}{l}\text { Survivors: } 75.3 \text { hours (median) (range 23-234 } \\
\text { hours) }\end{array}$ & $45(66.2 \%)$ & $22(32.4 \%)$ & $\begin{array}{l}22(32.4 \%) \\
\text { (2-year survival) }\end{array}$ & $\begin{array}{l}\text { Univentricular physiology, acute renal failure, duration of } \\
\text { ECMO, lowest lactate }\end{array}$ \\
\hline Ghez $2^{\mathrm{n}}-2005$ & $145 \pm 72$ hours $($ mean $\pm S D)$ & $13(87 \%)$ & $12(80 \%)$ & NA & NA \\
\hline Mahle $e^{12}-2005$ & $5.1 \pm 4.1$ days & Na & $16(50 \%)$ & $15(47 \%)$ & NA \\
\hline Baslaim $^{\mathrm{s}}-2006$ & Survivors: 74.5 hours (mean) (range $12-189$ hours) & Na & $12(46 \%)$ & NA & Stroke, DIC, renal failure \\
\hline Thouraniz ${ }^{23}-2006$ & $\begin{array}{l}151.5 \pm 201.4 \text { hours } \\
\text { (18-986 hours) }\end{array}$ & $20(74,1 \%)$ & $16(59.3 \%)$ & $13(48.1 \%)$ & NA \\
\hline Allan ${ }^{41}-2007$ & $\begin{array}{l}\text { Survivors } 56 \pm 15 \text { hours } \\
\text { Non-survivors } 160 \pm 23\end{array}$ & $30(68.2 \%)$ & $21(48 \%)$ & $12(27.2 \%)$ & 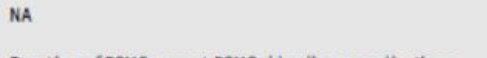 \\
\hline Alsoufit ${ }^{42}-2009$ & Survivors: 3 days (median) (range 2.8-4.2 days) & $109(61 \%)$ & $68(38 \%)$ & NA & $\begin{array}{l}\text { Duration of ECMO, repeat ECMO, bleeding complications, } \\
\text { neurological complications, renal dysfunction }\end{array}$ \\
\hline Delmo Walter ${ }^{21}-2010$ & $4.97 \pm 0.68$ days $($ mean $\pm S D)$ & $18(66.7 \%)$ & $9(33.3 \%)$ & NA & Duration of CPR, High doses of inotropes \\
\hline Polimenakos ${ }^{27}-2011$ & 7 days (median) (IOR 4-21 days) & $15(72 \%)$ & $13(62 \%)$ & $10(47 \%)$ & $\begin{array}{l}\text { High serum peak lactate (first } 24 \text { hours), longer ECMO } \\
\text { duration }\end{array}$ \\
\hline Bhat ${ }^{36}-2013$ & $\begin{array}{l}\text { Total: } 164 \text { hours (median) (10R } 95-231 \text { ) } \\
\text { Survivors: } 134 \text { hours (IQR } 95 \text {-160 hours) }\end{array}$ & $33(52 \%)$ & $18(28.1 \%)$ & NA & $\begin{array}{l}\text { Renal replacement ther apy on ECMO, ECM0 duration } \\
>231 \text { hours }\end{array}$ \\
\hline Sasson $^{23} \cdot 2013$ & $\begin{array}{l}\text { Total: } 4 \text { days (median) (range } 1-13 \text { days) } \\
\text { Survivors: } 3 \text { days (range } 1-13 \text { days) }\end{array}$ & $29(46.8 \%)$ & $24(38.7 \%)$ & NA & Total anomalous pulmonary venous return \\
\hline Aganwal ${ }^{16}-2014$ & 4 days (median) (10R 2-7 days) & $75(63 \%)$ & $49(41 \%)$ & NA & NA \\
\hline Alsoufit ${ }^{3}-2014$ & $\begin{array}{l}\text { Total: } 4 \text { days (median) (IQR } 3-6 \text { days) } \\
\text { Survivors: } 3 \text { days (IQR 2-5 days) }\end{array}$ & $62(62 \%)$ & $37(37 \%)$ & NA & $\begin{array}{l}\text { Renal failure requiring dialysis, maximum creatinine, } \\
\text { bleeding requining re-exploration, ECM0 duration, hours } \\
\text { to lactate normalization, immediate post-EOMO lactate, } \\
\text { peak post-EOMO lactate, maximum bilirubin, sepsis }\end{array}$ \\
\hline Sasaki $^{37}-2014$ & $4.9 \pm 4.2$ days $($ mean \pm SD) & $21(58 \%)$ & $17(47 \%)$ & NA & $\begin{array}{l}\text { Univentricular anatomy, younger age, longer ECMO } \\
\text { duration, higher lactate, pulmonary hemorrhage }\end{array}$ \\
\hline Jolley ${ }^{45}-2014$ & $\begin{array}{l}\text { Survivors: } 88 \text { hours ( } 48-132) \\
\text { Non-survivors: } 136 \text { hours }(73-267)\end{array}$ & $68(66 \%)$ & $42(41 \%)$ & NA & $\begin{array}{l}\text { Inotrope requirement, longer duration of ECMO support, com- } \\
\text { bined cardopulmonary indication for ECMO, and renal failure }\end{array}$ \\
\hline Miana $^{24}-2015$ & $182.2 \pm 117$ hours $($ mean $\pm S D)$ & $26(46.4 \%)$ & $11(19.6 \%)$ & NA & NA \\
\hline Gupta $^{22}-2015$ & 4 days (median) (IOR 1-7 days) & NA & $518(51.9 \%)$ & NA & Longer EOM0 duration \\
\hline $\operatorname{Lou}^{15}-2015$ & $\begin{array}{l}\text { Group } 1 \text { (ECM0 without therapeutic hypothermia): } \\
83 \text { hours (med ian) (range 26-332 hours) } \\
\text { Group } 2 \text { (ECM0 with therapeutic hypothermia): } \\
106 \text { hours (median) (range 24-367 hours) }\end{array}$ & $77(80.2 \%)$ & $55(57.3 \%)$ & NA & NA \\
\hline Sznycer-Taub ${ }^{23}-2016$ & $\begin{array}{l}\text { Total: } 5 \text { days (median) (IQR } 3-7 \text { days) } \\
\text { 30-day survivors: } \\
4 \text { days (IOR } 3-6 \text { days) }\end{array}$ & NA & $46(49 \%)$ & NA & $\begin{array}{l}\text { Longer EOM0 duration, high mean } \mathrm{PaO}_{2} \text { (>193 mm Hg) } \\
\text { (first } 48 \text { hours) }\end{array}$ \\
\hline Aydin $^{11}-2016$ & $\begin{array}{l}\text { Sunvivors: } 88.5 \text { hours }(57-116) \\
\text { Non-survivors: } 183 \text { hours }(71-288)\end{array}$ & NA & $46(48 \%)$ & NA & $\begin{array}{l}\text { The duration of intubation, partial pressure carbon } \\
\text { dioxide, mean airway pressure, and renal injury }\end{array}$ \\
\hline Howard ${ }^{4}-2016$ & $7.5(3-11)$ days & NA & $42(50 \%)$ & $34(40 \%)$ & $\begin{array}{l}\text { Prematurity, } \mathrm{pH}<7.17 \text { pre-EOM0, inotropic support, ECM0 } \\
\text { duration }>168 \text { hours }\end{array}$ \\
\hline EMahrouk ${ }^{56}-2017$ & 4 days (median) (range $1-14$ days) & $53(47 \%)$ & $42(37 \%)$ & NA & Longer EOM 0 duration, renal failure, stroke \\
\hline Mistry ${ }^{27}-2018$ & 126.97 hours $(78.89-216.64)$ & NA & $48(70.6 \%)$ & $48(69.2 \%)^{3}$ & $\begin{array}{l}\text { Low body weight, serum lactate and creatinine, prior } \\
\text { cardiac surgery, inotroper use. }\end{array}$ \\
\hline
\end{tabular}

\section{Predictors of mortality}


Many studies have identified similar predictors of in-hospital mortality, summarized by Walters and colleagues to include longer CPB time, the inability to separate from CPB, elevated blood nitrogen urea 48 hours after ECMO cannulation, elevated creatinine 48 hours after ECMO cannulation, the need for red blood cells or plasma, and elevated right atrial pressure 8 hours after ECMO decannulation (Table 3). ${ }^{29}$ Kolovos and colleagues $^{18}$ found that CPR during ECMO cannulation, renal failure/dialysis, single ventricle palliated circulation, and a lactate trend within 48 hours of ECMO initiation to be associated with in-hospital mortality. Alsoufi and colleagues ${ }^{42,43}$ found that duration of ECMO, repeat ECMO, neurological complications, renal dysfunction, and mechanical complications were associated with in-hospital mortality. In Bhat's study of PCECMO neonates, renal replacement therapy on ECMO and duration of ECMO support $>231$ hours were predictors of poor prognosis. ${ }^{35}$ Renal failure is commonly associated with poorer prognosis in pediatric patients receiving PC-ECMO, which is in line with studies conducted in adults. ${ }^{44}$ Of note, continuous renal replacement therapy (CRRT) before ECMO is associated with worse outcomes than either no CRRT or CRRT started after ECMO. The former is indicative of underlying renal disease, while the latter may reflect an attempt to prevent fluid overload. ${ }^{45}$ Additionally, worse outcomes may be expected in patients with palliated single ventricle circulation with Glenn or Fontan operations, due to the ineffectiveness of conventional CPR and the high risk of brain injury unless the bidirectional cavopulmonary connection is cannulated along with the inferior vena cava (ie. bicaval or central cannulation may be required). ${ }^{36,46}$

\section{ECMO for extracorporeal cardiopulmonary resuscitation}

Extracorporeal cardiopulmonary resuscitation (ECPR), or ECMO to support CPA, is an important and increasing use of ECMO in children who undergo cardiac surgery. Nonetheless, early institution of ECMO support prior to CPA in children with deteriorating hemodynamics in the postoperative period is preferable. In a study of 81 children supported with ECMO following cardiac surgery for $\mathrm{CHD}$, Chaturvedi and colleagues reported that patients who underwent ECMO deployment in the operating room had improved survival to hospital discharge as compared with patients with ECMO was deployed in the intensive care unit (64\% vs. $29 \%$ ) suggesting that early institution of ECMO prevented exposure to a prolonged period of low cardiac output. 33

The ELSO International Summary, January 2019 shows similar survival to discharge for neonates supported with ECMO for ECPR as compared with ECMO support for other cardiac indications (41\% vs. 
$42 \%$ ), but lower survival to discharge for pediatric ECPR (42\%) as compared to ECMO for other cardiac indications (52\%) once the neonatal period is complete. Other studies have reported similar survival for ECPR and non-ECPR ECMO. ${ }^{47-49}$ Notably, the risk of neurological injury may be higher in children supported with ECPR ${ }^{48,}{ }^{49}$ Survival-to-discharge for both ECPR and non-ECPR ECMO patients is heavily influenced by the ability to reverse the postoperative cardiorespiratory failure that necessitated ECMO support.

\section{Complications of Post-Cardiotomy ECMO in Pediatric Patients}

Important complications of PC-ECMO in children and neonates include bleeding, mechanical complications, liver failure, sepsis, central nervous system events, and renal failure which are reported at different rates in various studies (Table 4). ${ }^{17,50,33}$ Mechanical complications of ECMO are also common. ${ }^{33}$

Neurological sequalae occur frequently in children supported with ECMO. In a study of 90 patients by Chow and colleagues, ${ }^{51}$ only 15 children survived without neurological sequelae. There were short-term neurological events (22\%) and long-term neurological sequelae (12\%), accounting for $39 \%$ of survivors. In a study of 1,898 neonates with CHD reported to the ELSO Registry, $14 \%$ suffered a neurological injury. ${ }^{38}$ Risk factors for neurological injury included birth weight $<3 \mathrm{~kg}$, $\mathrm{pH}<7.15$ pre-ECMO, and the need for CPR prior to ECMO. Importantly, the patients who suffered neurological injury had higher in-hospital mortality (73\%) as compared with those without neurological complications (53\%). Khan and colleagues ${ }^{52}$ reported that $17.5 \%$ of neonates supported on ECMO had intraventricular hemorrhage detected by cranial ultrasound (CUS). The investigators performed routine daily CUS on all neonatal patients and found that almost all intracranial hemorrhages occurred in the first 5 days after surgery (including pre-ECMO), and any hemorrhage after that time was associated with clinical symptoms. In infants with an open fontanelle, CUS is a safe bedside screening tool, which can be performed regularly when increased vigilance for neurological complications after ECMO cannulation is warranted.

Although femoral cannulation is less common than carotid cannulation in pediatric patients, when the femoral approach is used, limb ischemia may be a serious complication. ${ }^{53}$ Methods to prevent limb ischemia in this setting include the use of contralateral femoral vessels for arterial and venous cannulation, the use of the smallest arterial cannula for the desired flow rate, incorporation of a chimney graft, and the use of a distal reperfusion cannula in an antegrade manner in the femoral artery or in a retrograde manner in the dorsalis 
pedis artery. In a single-center study of 29 children with femoral cannulation for V-A ECMO, Schad and colleagues $^{54}$ found that $29 \%$ of those without routine distal perfusion catheter placement suffered ischemic complications, compared with only $12 \%$ when distal perfusion catheters were routinely placed. In addition, non-invasive limb perfusion monitoring with near infrared spectroscopy has translated to better outcomes. ${ }^{55}$

Table 4 EQMO Complications

\begin{tabular}{|c|c|c|c|c|c|c|}
\hline Study & $\begin{array}{l}\text { Bleeding, } \\
\mathrm{n}(\%)\end{array}$ & $\begin{array}{l}\text { ECMO system } \\
\text { failure, } \mathrm{n}(\%)\end{array}$ & $\begin{array}{l}\text { Liver } \\
\text { failure, n (\%) }\end{array}$ & $\begin{array}{l}\text { Sepsis/infection/ } \\
\text { bacteremia, n (\%) }\end{array}$ & $\begin{array}{l}\text { CNS events, } \\
\mathrm{n}(\%)\end{array}$ & $\begin{array}{l}\text { Kidney failure, } \\
\text { n }(\%)\end{array}$ \\
\hline Klein $^{17}$ - 1990 & $17(47 \%)$ & NA & NA & NA & NA & NA \\
\hline Ferrazzi $i^{75}-1991$ & $6(100 \%)$ & NA & NA & $3(50 \%)$ & $1(17 \%)$ & NA \\
\hline Del Nido ${ }^{76}-1992$ & $1(9 \%)$ & NA & NA & $1(9 \%)$ & $3(27 \%)$ & NA \\
\hline Raithel $^{n}-1992$ & $44(68 \%)$ & $7(11 \%)$ & NA & $20(31 \%)$ & $18(28 \%)$ & $7(11 \%)$ \\
\hline Ziomek $^{10}-1992$ & NA & $3(13 \%)$ & NA & $8(33 \%)$ & $1(4 \%)$ & $4(17 \%)$ \\
\hline Dalton ${ }^{23}-1993$ & $6(22 \%)$ & NA & NA & $3(11 \%)$ & $6(22 \%)$ & NA \\
\hline Walters $^{20}-1995$ & $5(13 \%)$ & NA & $2(5 \%)$ & $4(10 \%)$ & $9(24 \%)$ & $3(8 \%)$ \\
\hline Kulik ${ }^{50}-1996$ & $28(44 \%)$ & NA & $18(28 \%)$ & $12(19 \%)$ & $29(45 \%)$ & $28(44 \%)$ \\
\hline Langley ${ }^{6}-1998$ & $4(44 \%)$ & NA & NA & $5(56 \%)$ & $1(11 \%)$ & $7(78 \%)$ \\
\hline Jaggers ${ }^{70}-2000$ & $15(35 \%)$ & NA & NA & $9(26 \%)$ & $18(51 \%)$ & $9(26 \%)$ \\
\hline Aharon ${ }^{80}-2001$ & NA & NA & NA & $7(14 \%)$ & NA & $4(8 \%)$ \\
\hline Pizarro $^{68}-2001$ & $2(17 \%)$ & $1(8 \%)$ & NA & $5(42 \%)$ & $3(25 \%)$ & $5(42 \%)$ \\
\hline Kolovos ${ }^{18}-2003$ & NA & NA & NA & $11(15 \%)$ & $16(22 \%)$ & $26(35 \%)$ \\
\hline Chaturvedi $i^{33}-2004$ & $51(63 \%)$ & $22(27 \%)$ & NA & $22(27 \%)$ & NA & $22(27 \%)$ \\
\hline Chow $w^{51}-2004$ & NA & NA & NA & NA & $20(22 \%)$ & NA \\
\hline Morris $^{26}-2004$ & $31(35 \%)$ & NA & NA & NA & $10(19 \%)^{2}$ & NA \\
\hline Huang $^{34}-2005$ & $34(50 \%)$ & NA & NA & NA & NA & $47(69 \%)$ \\
\hline Ghez $z^{\text {mi }}-2005$ & NA & NA & NA & $2(13 \%)$ & $0(0 \%)$ & $6(40 \%)$ \\
\hline Mahle $e^{71}-2005$ & NA & $1(3.1 \%)$ & NA & $1(3.1 \%)$ & $7(21.9 \%)$ & NA \\
\hline Baslaim $25-2006$ & $17(65 \%)$ & NA & NA & $4(15 \%)$ & $5(19 \%)$ & $8(31 \%)$ \\
\hline Allan ${ }^{41}-2008$ & NA & NA & NA & NA & $10(22.7 \%)$ & NA \\
\hline Alsoufi ${ }^{42}-2009$ & $100(56 \%)$ & $68(38 \%)$ & NA & NA & $32(18 \%)$ & $18(10 \%)$ \\
\hline Polimenakos $^{27}$ - 2011 & NA & NA & NA & $4(19 \%)$ & $9(43 \%)$ & $7(33 \%)$ \\
\hline Bhat $^{15}-2013$ & NA & NA & NA & NA & NA & $36(56 \%)$ \\
\hline Ag anwal ${ }^{16}-2014$ & NA & NA & NA & NA & NA & $41(34 \%)$ \\
\hline Alsoufi ${ }^{43} 2014$ & $49(49 \%)$ & $9(9 \%)$ & NA & $21(21 \%)$ & $17(17 \%)$ & $55(56 \%)$ \\
\hline Sasaki ${ }^{37}-2014$ & $17(47 \%)$ & $7(19 \%)$ & NA & $10(28 \%)$ & $10(28 \%)$ & $19(53 \%)$ \\
\hline Jolley ${ }^{48}-2014$ & $48(46 \%)$ & $46(44 \%)$ & $7(6 \%)$ & $12(11 \%)$ & $24(23 \%)$ & $34(33 \%)$ \\
\hline Miana $a^{34}-2015$ & $19(34 \%)$ & NA & NA & NA & $6(11 \%)$ & $5(9 \%)$ \\
\hline Lou $^{25}-2015$ & $29(30 \%)$ & $11(11 \%)$ & NA & $40(42 \%)$ & $11(11 \%)$ & $51(53 \%)$ \\
\hline Sznycer-Taub $^{23}-2016$ & NA & NA & NA & NA & $39(42 \%)$ & $35(38 \%)$ \\
\hline Aydin ${ }^{11}-2016$ & $27(30 \%)$ & $41(46 \%)$ & NA & $9(10 \%)$ & $15(16 \%)$ & $37(41 \%)$ \\
\hline Howard $d^{46}-2016$ & $32(43 \%)$ & $41(49 \%)$ & $16(19 \%)$ & NA & $21(25 \%)$ & $16(19 \%)$ \\
\hline ElMahrouk $^{86}-2017$ & $78(69 \%)$ & NA & NA & $35(31 \%)$ & $18(16 \%)$ & $41(36 \%)$ \\
\hline
\end{tabular}

\section{Post-Cardiotomy Veno-Venous ECMO for Respiratory Dysfunction in Pediatric Patients}

Despite advances in CPB techniques and in preventive measures aimed at decreasing respiratory complications after cardiac surgery, postoperative acute respiratory distress syndrome (ARDS) occurs in $1-20 \%$ of patients, depending on inclusion criteria. ${ }^{56-59}$ The use of V-A ECMO for refractory cardiovascular dysfunction after pediatric cardiac surgery has been described, but there is paucity of data on the use of 
postcardiotomy V-V ECMO. Respiratory distress and hypoxia are reported as indications for ECMO support in 2-30\% of pediatric patients. ${ }^{11,13-15} \mathrm{~V}-\mathrm{V}$ ECMO is an uncommon mode of support for patients with underlying cardiac disease, however in selected patients, it may be the mode of choice to facilitate oxygenation and decrease pulmonary vascular resistance. ${ }^{13}$

\section{Controversial Issues and Future Perspectives}

\section{Cardiac catherization during PC-ECMO}

Diagnostic or therapeutic cardiac catheterization can be safely performed on patients receiving ECMO support. ${ }^{60}$ Early detection and correction of residual cardiac lesions is associated with improved survival. ${ }^{16,61}$ Catheter-based diagnostic procedures should be considered when non-invasive diagnostic studies fail to identify a reason for failure to wean from ECMO and also, to evaluate decompression of the left side of the heart. ${ }^{62}$ Callahan and colleagues reported the results of cardiac catheterization on 36 pediatric patients supported by ECMO. ${ }^{61}$ They found that the catheterization investigation excluded a residual lesion in $18 \%$ of the patients, confirmed a residual lesion in $15 \%$, and identified unexpected residual cardiac lesions in $52 \%$. Interventions to manage the residual lesion were performed in $50 \%$ of cases, including stenting, device closure, or thrombolysis. After the cardiac catheterization procedure, $86 \%$ of patients were weaned from ECMO and $72 \%$ survived to discharge. Of note, catheter-based diagnostic procedures performed during the first or second day of ECMO support (day 0 or 1) significantly reduced the duration of ECMO without impacting survival. Recently, Another single center report of cardiac catheterization on 51 children on ECMO support demonstrated a low rate of serious complications (5.6\%), and subsequent decannulation/weaning and survival rates were $71 \%$ and $54 \%$, respectively. ${ }^{63}$ These studies demonstrate the benefit of cardiac catheterization in evaluating PC-ECMO-supported patient, despite the complexity of interpreting hemodynamic measurements with ECMO cannulae in situ, with or without cessation of ECMO flow for the procedure. Transport of the ECMO patient for cardiac catheterization was reported as uncomplicated, and overall, the complication rate was low. ${ }^{61,63}$

\section{Decompression of the left side of the heart}

Assessment and management of left heart decompression is a common indication for cardiac catheterization. ${ }^{61,63}$ Left heart hypertension of patients managed on ECMO can be addressed by atrial 
septostomy, use of an axial trans-aortic valve pump (Impella), direct left ventricular venting via an open approach, or with left atrial cannulation, either directly or via catheter crossing the atrial septum. Eastaugh and colleagues reported percutaneous left heart decompression in 44 of 419 patients managed on V-A ECMO, via atrial septostomy, stenting of the atrial septum or left atrial venting across the atrial septum. ${ }^{64}$ All techniques were equally successful at reducing left atrial pressure and decreasing pulmonary edema. Another single center study reported left heart decompression in 49 children managed on central V-A ECMO with left atrial venting, atrial septostomy, and left ventricle cannulation. ${ }^{65}$ Elective left heart decompression was associated with reduced duration of ECMO support, but was not associated with improved survival. Recently, an Impella device was used to decompress the left ventricle of 4 children on V-A ECMO. ${ }^{66}$ The device reduced left atrial pressure and increased tissue perfusion as observed by near infrared spectroscopy. Institutional preferences for assessment, timing, and mechanism of left heart decompression vary.

\section{Single ventricle physiology}

Despite advances in care, mortality after ECMO support in patients with single ventricle palliated circulation remains greater than $50 \% .^{18,67,28}$ The higher mortality in this subset of patients with cardiac palliation has been hypothesized as an imbalance between systemic and pulmonary blood flow and associated suboptimal coronary perfusion and increased probability of ventricular distension. ${ }^{28,68}$ Indications for ECMO in pediatric patients with single ventricle palliated circulation are comparable to those in patients with biventricular circulation, but additional complications are associated with systemic-to-pulmonary artery shunts. ${ }^{62}$ When the pulmonary circulation is supplied by a systemic-to-pulmonary artery shunt and ECMO support is instituted, the shunt is most often left open. Adequate support of this circulation may require an augmented circuit blood flow of $150-200 \mathrm{ml} / \mathrm{Kg} / \mathrm{min}$ to allow for diastolic run-off to the pulmonary circulation. In patients with single ventricle palliated circulation, cannulation strategy is an important issue. ${ }^{32,41,48}$ Inhospital mortality rates with the use of ECMO after the Norwood single ventricle palliation procedure exceed 50\%. ${ }^{69}, 70$ ECMO support of patients with Glenn and Fontan circulation is associated with additional complexity due to the surgical anatomy and resulting physiology. Multiple drainage cannulae may be required to optimize support. ${ }^{32}$ In all stages of single-ventricle palliation, long-duration ECMO, inotropic support, and renal failure are associated with higher mortality. ${ }^{48,49}$ The adequacy of the cannulation strategy should be 
questioned if the ECMO-supported patient with a single ventricle palliated circulation has evidence of ongoing poor end-organ oxygen delivery.

\section{ECMO costs}

Against the background of high mortality prior to hospital discharge, the cost of ECMO support requires some consideration. Mahle and colleagues focused on hospital costs in 32 pediatric patients with CHD who received salvage ECMO (18 for cardiopulmonary arrest and 14 PC shock). ${ }^{71}$ Survival to hospital discharge was $50 \%$ and 1-year survival was $47 \%$. The quality-of-life of the survivors was determined with the Health Utilities Index Mark II, and median cost for hospital stay after institution of ECMO was $\$ 156,324$ per patient. The calculated cost-utility for salvage ECMO in this population was $\$ 24,386$ per quality-adjusted life-year saved, which would be considered within the range of accepted cost-efficacy $(<\$ 50,000$ per quality-adjusted life-year saved). The authors note, however, that although the hospital costs for salvage cardiac ECMO are similar to those for neonatal ECMO for noncardiac indications, the calculated cost-utility is slightly less favorable. Salvage cardiac ECMO may be somewhat less cost-effective than noncardiac ECMO since the survival to hospital discharge is lower for cardiac patients. In addition, life expectancy for children with complex CHD, such as those with single ventricle palliated circulation, is lower than for children with respiratory distress in the neonatal period. Other investigators also noted a greater cost was associated with smaller hospitals and hospital location. ${ }^{72}$

\section{Bridging to heart transplantation}

ECMO support for children with circulatory failure awaiting heart transplantation has been analyzed using combined data from the ELSO Registry and the United States Organ Procurement Transplant Network (OPTN). ${ }^{10}$ The authors demonstrated that ECMO was associated with high mortality while on the waiting list, and one-third of the patients who received a heart transplant died before hospital discharge. Overall, survival to hospital discharge of this population was $47 \%$. In this context, other forms of temporary MCS are often considered for children awaiting heart transplantation. ${ }^{73}$ While bridging to transplantation represents a different clinical challenge than PC-ECMO, these studies may lead to innovative techniques for post-cardiotomy circulatory support that will advance the field.

\section{Limitations of the Review}


Pediatric PC-ECMO, particularly in the setting of CHD, is a technically challenging but potentially life-saving mode of support. There is limited evidence to inform clinical practice. We acknowledge several limitations to the current systematic review that are inherent to retrospective, observational studies. Indeed, most of the studies assembled here are single-center case series reports, precluding statistical analyses and lacking the power to detect some clinically significant differences in outcome. Secondly, the analyses were seldom adjusted for underlying confounders, such as duration of ECMO, cannulation strategy (peripheral vs central), cannula types and ECMO weaning protocols. Finally, although studies with large data sets coming from clinical registries (STS, ELSO, OPTN and PHIS) are discussed in the text, they were not included in the tables. ${ }^{20,21,36,38,74}$

\section{Conclusion}

V-A ECMO is the optimal support technique in children with CHD and post-cardiotomy shock. ECMO facilitates augmented cardiac output and respiratory gas exchange to improve the metabolic status of both preoperative and post-cardiotomy patients. Although PC-ECMO can improve survival of this vulnerable population, mortality and morbidity remain high. Complications related to bleeding, thrombosis, and infections increase mortality and are major areas for improvement. Neurological injury and neurodevelopmental impairment are common in pediatric patients post PC-ECMO and reflect the severity of critical illness, complexity of cardiac surgery and complications of ECMO support. Bridging to cardiac transplantation can be successful if PC-ECMO does not lead to cardiac recovery, but the availability of organs and waiting list duration are ultimately factors in survival. The paucity of standardized care processes, informed patient selection, optimal timing of ECMO deployment, anticoagulation strategies or weaning protocols and the lack of long-term follow up of survivors after PC-ECMO demonstrated on systematic review informs the urgent need for further studies to inform best management of this high-risk population.

\section{References}


1. Cooper DS, Jacobs JP, Moore L, Stock A, Gaynor JW, Chancy T, Parpard M, Griffin DA, Owens T, Checchia PA, Thiagarajan RR, Spray TL and Ravishankar C. Cardiac extracorporeal life support: state of the art in 2007. Cardiology in the young. 2007;17 Suppl 2:104-15.

2. Baffes TG, Fridman JL, Bicoff JP and Whitehill JL. Extracorporeal circulation for support of palliative cardiac surgery in infants. The Annals of thoracic surgery. 1970;10:354-63.

3. Soeter JR, Mamiya RT, Sprague AY and McNamara JJ. Prolonged extracorporeal oxygenation for cardiorespiratory failure after tetralogy correction. The Journal of thoracic and cardiovascular surgery. 1973;66:214-8.

4. Duncan BW. Pediatric mechanical circulatory support. ASAIO journal (American Society for Artificial Internal Organs : 1992). 2005;51:ix-xiv.

5. Chang AC, Wernovsky G, Kulik TJ, Jonas RA and Wessel DL. Management of the neonate with transposition of the great arteries and persistent pulmonary hypertension. The American journal of cardiology. 1991;68:1253-5.

6. Di Russo GB, Clark BJ, Bridges ND, Godinez RI, Paridon SM, Spray TL and Gaynor JW. Prolonged extracorporeal membrane oxygenation as a bridge to cardiac transplantation. The Annals of thoracic surgery. 2000;69:925-7.

7. Bautista-Hernandez V, Thiagarajan RR, Fynn-Thompson F, Rajagopal SK, Nento DE, Yarlagadda V, Teele SA, Allan CK, Emani SM, Laussen PC, Pigula FA and Bacha EA. Preoperative extracorporeal membrane oxygenation as a bridge to cardiac surgery in children with congenital heart disease. The Annals of thoracic surgery. 2009;88:1306-11.

8. Rossano JW, Cherikh WS, Chambers DC, Goldfarb S, Khush K, Kucheryavaya AY, Levvey BJ, Lund LH, Meiser B, Yusen RD and Stehlik J. The Registry of the International Society for Heart and Lung Transplantation: Twentieth Pediatric Heart Transplantation Report-2017; Focus Theme: 
Allograft ischemic time. The Journal of heart and lung transplantation : the official publication of the International Society for Heart Transplantation. 2017;36:1060-1069.

9. Lorts A, Eghtesady P, Mehegan M, Adachi I, Villa C, Davies R, Gossett JG, Kanter K, Alejos J, Koehl D, Cantor RS and Morales DLS. Outcomes of children supported with devices labeled as "temporary" or short term: A report from the Pediatric Interagency Registry for Mechanical Circulatory Support. The Journal of heart and lung transplantation : the official publication of the International Society for Heart Transplantation. 2018;37:54-60.

10. Almond CS, Singh TP, Gauvreau K, Piercey GE, Fynn-Thompson F, Rycus PT, Bartlett RH and Thiagarajan RR. Extracorporeal membrane oxygenation for bridge to heart transplantation among children in the United States: analysis of data from the Organ Procurement and Transplant Network and Extracorporeal Life Support Organization Registry. Circulation. 2011;123:2975-84.

11. Aydin SI, Duffy M, Rodriguez D, Rycus PT, Friedman P, Thiagarajan RR and Weinstein S. Venovenous extracorporeal membrane oxygenation for patients with single-ventricle anatomy: A registry report. The Journal of thoracic and cardiovascular surgery. 2016;151:1730-6.

12. Barbaro RP, Paden ML, Guner YS, Raman L, Ryerson LM, Alexander P, Nasr VG, Bembea MM, Rycus PT, Thiagarajan RR and centers Em. Pediatric Extracorporeal Life Support Organization Registry International Report 2016. ASAIO journal (American Society for Artificial Internal Organs : 1992). 2017;63:456-463.

13. Dalton HJ, Siewers RD, Fuhrman BP, Del Nido P, Thompson AE, Shaver MG and Dowhy M. Extracorporeal membrane oxygenation for cardiac rescue in children with severe myocardial dysfunction. Critical care medicine. 1993;21:1020-8.

14. Dhillon R, Pearson GA, Firmin RK, Chan KC and Leanage R. Extracorporeal membrane oxygenation and the treatment of critical pulmonary hypertension in congenital heart disease. 
European journal of cardio-thoracic surgery : official journal of the European Association for Cardiothoracic Surgery. 1995;9:553-6.

15. Kim K, Mazor RL, Rycus PT and Brogan TV. Use of venovenous extracorporeal life support in pediatric patients for cardiac indications: a review of the Extracorporeal Life Support Organization registry. Pediatric critical care medicine : a journal of the Society of Critical Care Medicine and the World Federation of Pediatric Intensive and Critical Care Societies. 2012;13:285-9.

16. Agarwal HS, Hardison DC, Saville BR, Donahue BS, Lamb FS, Bichell DP and Harris ZL. Residual lesions in postoperative pediatric cardiac surgery patients receiving extracorporeal membrane oxygenation support. The Journal of thoracic and cardiovascular surgery. 2014;147:43441.

17. Klein MD, Shaheen KW, Whittlesey GC, Pinsky WW and Arciniegas E. Extracorporeal membrane oxygenation for the circulatory support of children after repair of congenital heart disease. The Journal of thoracic and cardiovascular surgery. 1990;100:498-505.

18. Kolovos NS, Bratton SL, Moler FW, Bove EL, Ohye RG, Bartlett RH and Kulik TJ. Outcome of pediatric patients treated with extracorporeal life support after cardiac surgery. The Annals of thoracic surgery. 2003;76:1435-41; discussion 1441-2.

19. Ziomek S, Harrell JE, Jr., Fasules JW, Faulkner SC, Chipman CW, Moss M, Frazier E and Van Devanter SH. Extracorporeal membrane oxygenation for cardiac failure after congenital heart operation. The Annals of thoracic surgery. 1992;54:861-7; discussion 867-8.

20. Bratton SL, Chan T, Barrett CS, Wilkes J, Ibsen LM and Thiagarajan RR. Metrics to Assess Extracorporeal Membrane Oxygenation Utilization in Pediatric Cardiac Surgery Programs. Pediatric critical care medicine : a journal of the Society of Critical Care Medicine and the World Federation of Pediatric Intensive and Critical Care Societies. 2017;18:779-786. 
21. Mascio CE, Austin EH, Jacobs JP, Jacobs ML, Wallace AS, He X and Pasquali SK. Perioperative mechanical circulatory support in children: An analysis of the Society of Thoracic Surgeons Congenital Heart Surgery Database. The Journal of thoracic and cardiovascular surgery. 2014;147:658-665.

22. Gupta P, DasGupta R, Best D, Chu CB, Elsalloukh H, Gossett JM, Imamura M and Butt W. Delayed extracorporeal membrane oxygenation in children after cardiac surgery: two-institution experience. Cardiology in the young. 2015;25:248-54.

23. Sznycer-Taub NR, Lowery R, Yu S, Owens ST, Hirsch-Romano JC and Owens GE. Hyperoxia Is Associated With Poor Outcomes in Pediatric Cardiac Patients Supported on Venoarterial Extracorporeal Membrane Oxygenation. Pediatric critical care medicine : a journal of the Society of Critical Care Medicine and the World Federation of Pediatric Intensive and Critical Care Societies. 2016;17:350-8.

24. Polimenakos AC, Rizzo V, El-Zein CF and Ilbawi MN. Post-cardiotomy Rescue Extracorporeal Cardiopulmonary Resuscitation in Neonates with Single Ventricle After Intractable Cardiac Arrest: Attrition After Hospital Discharge and Predictors of Outcome. Pediatric cardiology. 2017;38:314-323.

25. Baslaim G, Bashore J, Al-Malki F and Jamjoom A. Can the outcome of pediatric extracorporeal membrane oxygenation after cardiac surgery be predicted? Annals of thoracic and cardiovascular surgery : official journal of the Association of Thoracic and Cardiovascular Surgeons of Asia. 2006;12:21-7.

26. Morris MC, Ittenbach RF, Godinez RI, Portnoy JD, Tabbutt S, Hanna BD, Hoffman TM, Gaynor JW, Connelly JT, Helfaer MA, Spray TL and Wernovsky G. Risk factors for mortality in 137 pediatric cardiac intensive care unit patients managed with extracorporeal membrane oxygenation. Critical care medicine. 2004;32:1061-9. 
27. Polimenakos AC, Wojtyla P, Smith PJ, Rizzo V, Nater M, El Zein CF and Ilbawi MN. Postcardiotomy extracorporeal cardiopulmonary resuscitation in neonates with complex single ventricle: analysis of outcomes. European journal of cardio-thoracic surgery : official journal of the European Association for Cardio-thoracic Surgery. 2011;40:1396-405; discussion 1405.

28. Thourani VH, Kirshbom PM, Kanter KR, Simsic J, Kogon BE, Wagoner S, Dykes F, Fortenberry $\mathbf{J}$ and Forbess JM. Venoarterial extracorporeal membrane oxygenation (VA-ECMO) in pediatric cardiac support. The Annals of thoracic surgery. 2006;82:138-44; discussion 144-5.

29. Walters HL, 3rd, Hakimi M, Rice MD, Lyons JM, Whittlesey GC and Klein MD. Pediatric cardiac surgical ECMO: multivariate analysis of risk factors for hospital death. The Annals of thoracic surgery. 1995;60:329-36; discussion 336-7.

30. Johnson K, Jarboe MD, Mychaliska GB, Barbaro RP, Rycus P, Hirschl RB, Gadepalli SK and Group ELE-ENOW. Is there a best approach for extracorporeal life support cannulation: a review of the extracorporeal life support organization. J Pediatr Surg. 2018;53:1301-1304.

31. Chan T, Thiagarajan RR, Frank D and Bratton SL. Survival after extracorporeal cardiopulmonary resuscitation in infants and children with heart disease. The Journal of thoracic and cardiovascular surgery. 2008;136:984-92.

32. Marino BS, Tabbutt S, MacLaren G, Hazinski MF, Adatia I, Atkins DL, Checchia PA, DeCaen A, Fink EL, Hoffman GM, Jefferies JL, Kleinman M, Krawczeski CD, Licht DJ, Macrae D, Ravishankar C, Samson RA, Thiagarajan RR, Toms R, Tweddell J, Laussen PC, American Heart Association Congenital Cardiac Defects Committee of the Council on Cardiovascular Disease in the Y, Council on Clinical C, Council on C, Stroke N, Council on Cardiovascular S, Anesthesia and Emergency Cardiovascular Care C. Cardiopulmonary Resuscitation in Infants and Children With Cardiac Disease: A Scientific Statement From the American Heart Association. Circulation. 2018;137:e691-e782. 
33. Chaturvedi RR, Macrae D, Brown KL, Schindler M, Smith EC, Davis KB, Cohen G, Tsang V, Elliott M, de Leval M, Gallivan S and Goldman AP. Cardiac ECMO for biventricular hearts after paediatric open heart surgery. Heart (British Cardiac Society). 2004;90:545-51.

34. Huang SC, Wu ET, Chen YS, Chang CI, Chiu IS, Chi NH, Wu MH, Wang SS, Lin FY and Ko WJ. Experience with extracorporeal life support in pediatric patients after cardiac surgery. ASAIO journal (American Society for Artificial Internal Organs : 1992). 2005;51:517-21.

35. Bhat P, Hirsch JC, Gelehrter S, Cooley E, Donohue J, King K and Gajarski RJ. Outcomes of infants weighing three kilograms or less requiring extracorporeal membrane oxygenation after cardiac surgery. The Annals of thoracic surgery. 2013;95:656-61.

36. Ford MA, Gauvreau K, McMullan DM, Almodovar MC, Cooper DS, Rycus PT and Thiagarajan R. Factors Associated With Mortality in Neonates Requiring Extracorporeal Membrane Oxygenation for Cardiac Indications: Analysis of the Extracorporeal Life Support Organization Registry Data. Pediatric critical care medicine : a journal of the Society of Critical Care Medicine and the World Federation of Pediatric Intensive and Critical Care Societies. 2016;17:860-70.

37. Sasaki T, Asou T, Takeda Y, Onakatomi Y, Tominaga T and Yamamoto Y. Extracorporeal life support after cardiac surgery in children: outcomes from a single institution. Artificial organs. 2014;38:34-40.

38. Polito A, Barrett CS, Rycus PT, Favia I, Cogo PE and Thiagarajan RR. Neurologic injury in neonates with congenital heart disease during extracorporeal membrane oxygenation: an analysis of extracorporeal life support organization registry data. ASAIO journal (American Society for Artificial Internal Organs : 1992). 2015;61:43-8.

39. McMullan DM, Thiagarajan RR, Smith KM, Rycus PT and Brogan TV. Extracorporeal cardiopulmonary resuscitation outcomes in term and premature neonates*. Pediatric critical care 
medicine : a journal of the Society of Critical Care Medicine and the World Federation of Pediatric Intensive and Critical Care Societies. 2014;15:e9-e16.

40. Gupta P, Robertson MJ, Beam B, Gossett JM, Schmitz ML, Carroll CL, Edwards JD, Fortenberry JD and Butt W. Relationship of ECMO duration with outcomes after pediatric cardiac surgery: a multi-institutional analysis. Minerva anestesiologica. 2015;81:619-27.

41. Allan CK, Thiagarajan RR, del Nido PJ, Roth SJ, Almodovar MC and Laussen PC. Indication for initiation of mechanical circulatory support impacts survival of infants with shunted singleventricle circulation supported with extracorporeal membrane oxygenation. The Journal of thoracic and cardiovascular surgery. 2007;133:660-7.

42. Alsoufi B, Al-Radi OO, Gruenwald C, Lean L, Williams WG, McCrindle BW, Caldarone CA and Van Arsdell GS. Extra-corporeal life support following cardiac surgery in children: analysis of risk factors and survival in a single institution. European journal of cardio-thoracic surgery : official journal of the European Association for Cardio-thoracic Surgery. 2009;35:1004-11; discussion 1011.

43. Alsoufi B, Awan A, Manlhiot C, Al-Halees Z, Al-Ahmadi M, McCrindle BW and Alwadai A. Does single ventricle physiology affect survival of children requiring extracorporeal membrane oxygenation support following cardiac surgery? World journal for pediatric \& congenital heart surgery. 2014;5:7-15.

44. Yan X, Jia S, Meng X, Dong P, Jia M, Wan J and Hou X. Acute kidney injury in adult postcardiotomy patients with extracorporeal membrane oxygenation: evaluation of the RIFLE classification and the Acute Kidney Injury Network criteria. European journal of cardio-thoracic surgery : official journal of the European Association for Cardio-thoracic Surgery. 2010;37:334-8.

45. Haneya A, Diez C, Philipp A, Bein T, Mueller T, Schmid C and Lubnow M. Impact of Acute Kidney Injury on Outcome in Patients With Severe Acute Respiratory Failure Receiving Extracorporeal Membrane Oxygenation. Critical care medicine. 2015;43:1898-906. 
46. Howard TS, Kalish BT, Wigmore D, Nathan M, Kulik TJ, Kaza AK, Williams K and Thiagarajan RR. Association of Extracorporeal Membrane Oxygenation Support Adequacy and Residual Lesions With Outcomes in Neonates Supported After Cardiac Surgery. Pediatric critical care medicine : a journal of the Society of Critical Care Medicine and the World Federation of Pediatric Intensive and Critical Care Societies. 2016;17:1045-1054.

47. Chrysostomou C, Morell VO, Kuch BA, O'Malley E, Munoz R and Wearden PD. Short- and intermediate-term survival after extracorporeal membrane oxygenation in children with cardiac disease. The Journal of thoracic and cardiovascular surgery. 2013;146:317-25.

48. Jolley M, Thiagarajan RR, Barrett CS, Salvin JW, Cooper DS, Rycus PT and Teele SA. Extracorporeal membrane oxygenation in patients undergoing superior cavopulmonary anastomosis. The Journal of thoracic and cardiovascular surgery. 2014;148:1512-8.

49. Rood KL, Teele SA, Barrett CS, Salvin JW, Rycus PT, Fynn-Thompson F, Laussen PC and Thiagarajan RR. Extracorporeal membrane oxygenation support after the Fontan operation. The Journal of thoracic and cardiovascular surgery. 2011;142:504-10.

50. Kulik TJ, Moler FW, Palmisano JM, Custer JR, Mosca RS, Bove EL and Bartlett RH. Outcome-associated factors in pediatric patients treated with extracorporeal membrane oxygenator after cardiac surgery. Circulation. 1996;94:Ii63-8.

51. Chow G, Koirala B, Armstrong D, McCrindle B, Bohn D, Edgell D, Coles J and de Veber G. Predictors of mortality and neurological morbidity in children undergoing extracorporeal life support for cardiac disease. European journal of cardio-thoracic surgery : official journal of the European Association for Cardio-thoracic Surgery. 2004;26:38-43.

52. Khan AM, Shabarek FM, Zwischenberger JB, Warner BW, Cheu HW, Jaksic T, Goretsky MJ, Meyer TA, Doski J and Lally KP. Utility of daily head ultrasonography for infants on extracorporeal membrane oxygenation. J Pediatr Surg. 1998;33:1229-32. 
53. Fraser CD, 3rd, Kovler ML, Guzman W, Jr., Rhee DS, Lum YW, Alaish SM and Garcia AV. Pediatric Femoral Arterial Cannulations in Extracorporeal Membrane Oxygenation: A Review and Strategies for Optimization. ASAIO journal (American Society for Artificial Internal Organs : 1992). 2018.

54. Schad CA, Fallon BP, Monteagudo J, Okochi S, Cheung EW, Morrissey NJ, KadenheChiweshe AV, Aspelund G, Stylianos S and Middlesworth W. Routine Use of Distal Arterial Perfusion in Pediatric Femoral Venoarterial Extracorporeal Membrane Oxygenation. Artificial organs. 2017;41:11-16.

55. Kim DJ, Cho YJ, Park SH, Lim C, Park KH, Jheon S and Kim JS. Near-Infrared Spectroscopy Monitoring for Early Detection of Limb Ischemia in Patients on Veno-Arterial Extracorporeal Membrane Oxygenation. ASAIO journal (American Society for Artificial Internal Organs : 1992). 2017;63:613-617.

56. Stephens RS, Shah AS and Whitman GJ. Lung injury and acute respiratory distress syndrome after cardiac surgery. The Annals of thoracic surgery. 2013;95:1122-9.

57. Rahmanian PB, Adams DH, Castillo JG, Carpentier A and Filsoufi F. Predicting hospital mortality and analysis of long-term survival after major noncardiac complications in cardiac surgery patients. The Annals of thoracic surgery. 2010;90:1221-9.

58. Rong LQ, Di Franco A and Gaudino M. Acute respiratory distress syndrome after cardiac surgery. J Thorac Dis. 2016;8:E1177-E1186.

59. Peek GJ, Mugford M, Tiruvoipati R, Wilson A, Allen E, Thalanany MM, Hibbert CL, Truesdale A, Clemens F, Cooper N, Firmin RK and Elbourne D. Efficacy and economic assessment of conventional ventilatory support versus extracorporeal membrane oxygenation for severe adult respiratory failure (CESAR): a multicentre randomised controlled trial. Lancet (London, England). 2009;374:1351-63. 
60. Booth KL, Roth SJ, Perry SB, del Nido PJ, Wessel DL and Laussen PC. Cardiac catheterization of patients supported by extracorporeal membrane oxygenation. Journal of the American College of Cardiology. 2002;40:1681-6.

61. Callahan R, Trucco SM, Wearden PD, Beerman LB, Arora G and Kreutzer J. Outcomes of pediatric patients undergoing cardiac catheterization while on extracorporeal membrane oxygenation. Pediatric cardiology. 2015;36:625-32.

62. Di Nardo M, MacLaren G, Marano M, Cecchetti C, Bernaschi P and Amodeo A. ECLS in Pediatric Cardiac Patients. Frontiers in pediatrics. 2016;4:109.

63. Boscamp NS, Turner ME, Crystal M, Anderson B, Vincent JA and Torres AJ. Cardiac Catheterization in Pediatric Patients Supported by Extracorporeal Membrane Oxygenation: A 15Year Experience. Pediatric cardiology. 2017;38:332-337.

64. Eastaugh LJ, Thiagarajan RR, Darst JR, McElhinney DB, Lock JE and Marshall AC. Percutaneous left atrial decompression in patients supported with extracorporeal membrane oxygenation for cardiac disease. Pediatric critical care medicine : a journal of the Society of Critical Care Medicine and the World Federation of Pediatric Intensive and Critical Care Societies. 2015;16:59-65.

65. Hacking DF, Best D, d'Udekem Y, Brizard CP, Konstantinov IE, Millar J and Butt W. Elective decompression of the left ventricle in pediatric patients may reduce the duration of venoarterial extracorporeal membrane oxygenation. Artificial organs. 2015;39:319-26.

66. Parekh D, Jeewa A, Tume SC, Dreyer WJ, Pignatelli R, Horne D, Justino H and Qureshi AM. Percutaneous Mechanical Circulatory Support Using Impella Devices for Decompensated Cardiogenic Shock: A Pediatric Heart Center Experience. ASAIO journal (American Society for Artificial Internal Organs : 1992). 2018;64:98-104. 
67. Langley SM, Sheppard SV, Tsang VT, Monro JL and Lamb RK. When is extracorporeal life support worthwhile following repair of congenital heart disease in children? European journal of cardio-thoracic surgery : official journal of the European Association for Cardio-thoracic Surgery. 1998;13:520-5.

68. Pizarro C, Davis DA, Healy RM, Kerins PJ and Norwood WI. Is there a role for extracorporeal life support after stage I Norwood? European journal of cardio-thoracic surgery : official journal of the European Association for Cardio-thoracic Surgery. 2001;19:294-301.

69. Friedland-Little JM, Aiyagari R, Yu S, Donohue JE and Hirsch-Romano JC. Survival through staged palliation: fate of infants supported by extracorporeal membrane oxygenation after the Norwood operation. The Annals of thoracic surgery. 2014;97:659-65.

70. Tabbutt S, Ghanayem N, Ravishankar C, Sleeper LA, Cooper DS, Frank DU, Lu M, Pizarro C, Frommelt P, Goldberg CS, Graham EM, Krawczeski CD, Lai WW, Lewis A, Kirsh JA, Mahony L, Ohye RG, Simsic J, Lodge AJ, Spurrier E, Stylianou M, Laussen P and Pediatric Heart Network I. Risk factors for hospital morbidity and mortality after the Norwood procedure: A report from the Pediatric Heart Network Single Ventricle Reconstruction trial. The Journal of thoracic and cardiovascular surgery. 2012;144:882-95.

71. Mahle WT, Forbess JM, Kirshbom PM, Cuadrado AR, Simsic JM and Kanter KR. Cost-utility analysis of salvage cardiac extracorporeal membrane oxygenation in children. The Journal of thoracic and cardiovascular surgery. 2005;129:1084-90.

72. Faraoni D, Nasr VG, DiNardo JA and Thiagarajan RR. Hospital Costs for Neonates and Children Supported with Extracorporeal Membrane Oxygenation. The Journal of pediatrics. 2016;169:69-75 e1.

73. Yarlagadda VV, Maeda K, Zhang Y, Chen S, Dykes JC, Gowen MA, Shuttleworth P, Murray JM, Shin AY, Reinhartz O, Rosenthal DN, McElhinney DB and Almond CS. Temporary Circulatory 
Support in U.S. Children Awaiting Heart Transplantation. Journal of the American College of Cardiology. 2017;70:2250-2260.

74. Barrett CS, Chan TT, Wilkes J, Bratton SL and Thiagarajan RR. Association of Pediatric Cardiac Surgical Volume and Mortality After Cardiac ECMO. ASAIO journal (American Society for Artificial Internal Organs : 1992). 2017;63:802-809.

75. Ferrazzi P, Glauber M, Di Domenico A, Fiocchi R, Mamprin F, Gamba A, Crupi G, Cossolini M and Parenzan L. Assisted circulation for myocardial recovery after repair of congenital heart disease. European journal of cardio-thoracic surgery : official journal of the European Association for Cardio-thoracic Surgery. 1991;5:419-23; discussion 424.

76. del Nido PJ, Dalton HJ, Thompson AE and Siewers RD. Extracorporeal membrane oxygenator rescue in children during cardiac arrest after cardiac surgery. Circulation. 1992;86:Ii3004.

77. Raithel SC, Pennington DG, Boegner E, Fiore A and Weber TR. Extracorporeal membrane oxygenation in children after cardiac surgery. Circulation. 1992;86:Ii305-10.

78. Black MD, Coles JG, Williams WG, Rebeyka IM, Trusler GA, Bohn D, Gruenwald C and Freedom RM. Determinants of success in pediatric cardiac patients undergoing extracorporeal membrane oxygenation. The Annals of thoracic surgery. 1995;60:133-8.

79. Jaggers JJ, Forbess JM, Shah AS, Meliones JN, Kirshbom PM, Miller CE and Ungerleider RM. Extracorporeal membrane oxygenation for infant postcardiotomy support: significance of shunt management. The Annals of thoracic surgery. 2000;69:1476-83.

80. Aharon AS, Drinkwater DC, Jr., Churchwell KB, Quisling SV, Reddy VS, Taylor M, Hix S, Christian KG, Pietsch JB, Deshpande JK, Kambam J, Graham TP and Chang PA. Extracorporeal membrane oxygenation in children after repair of congenital cardiac lesions. The Annals of thoracic surgery. 2001;72:2095-101; discussion 2101-2. 
81. Ghez O, Feier H, Ughetto F, Fraisse A, Kreitmann B and Metras D. Postoperative extracorporeal life support in pediatric cardiac surgery: recent results. ASAIO journal (American Society for Artificial Internal Organs : 1992). 2005;51:513-6.

82. Delmo Walter EM, Alexi-Meskishvili V, Huebler M, Loforte A, Stiller B, Weng Y, Berger F and Hetzer R. Extracorporeal membrane oxygenation for intraoperative cardiac support in children with congenital heart disease. Interactive cardiovascular and thoracic surgery. 2010;10:753-8.

83. Sasson L, Cohen I, Tamir A, Sternfeld AR, Berlowitz Y, Lenczner O and Houri S. Extracorporeal membrane oxygenation in pediatric patients: our experience in the last ten years. The Israel Medical Association journal : IMAJ. 2013;15:13-6.

84. Miana LA, Caneo LF, Tanamati C, Penha JG, Guimaraes VA, Miura N, Galas FR and Jatene MB. Post-cardiotomy ECMO in pediatric and congenital heart surgery: impact of team training and equipment in the results. Revista brasileira de cirurgia cardiovascular : orgao oficial da Sociedade Brasileira de Cirurgia Cardiovascular. 2015;30:409-16.

85. Lou S, MacLaren G, Clark J, Paul E, Best D, Delzoppo C, d'Udekem Y and Butt W. Safety of therapeutic hypothermia in children on veno-arterial extracorporeal membrane oxygenation after cardiac surgery. Cardiology in the young. 2015;25:1367-73.

86. ElMahrouk AF, Ismail MF, Hamouda T, Shaikh R, Mahmoud A, Shihata MS, Alradi O and Jamjoom A. Extracorporeal Membrane Oxygenation in Postcardiotomy Pediatric Patients-15 Years of Experience Outside Europe and North America. The Thoracic and cardiovascular surgeon. 2017.

87. Mistry MS, Trucco SM, Maul T, Sharma MS, Wang L and West S. Predictors of Poor Outcomes in Pediatric Venoarterial Extracorporeal Membrane Oxygenation. World journal for pediatric \& congenital heart surgery. 2018:2150135118762391. 


\section{CHAPTER 8}

\section{Pulmonary Artery Cannulation to Enhance Extracorporeal Membrane Oxygenation}

Management in Acute Cardiac Failure

Lorusso R*, Raffa GM*, Heuts S, Lo Coco V, Meani P, Gilbers M, Natour E, Bidar E, Delnoij T, Maessen J, Loforte A

*Equally contributor

Interact Cardiovasc Thorac Surg. 2019 Oct 26. 


\begin{abstract}
Objective. Pulmonary artery (PA) cannulation during peripheral veno-arterial extracorporeal membrane oxygenation (V-A ECMO) has been shown to be effective either for indirect left ventricular (LV) unloading or to allow right ventricular (RV) bypass with associated gas-exchange support in case of acute RV with respiratory failure. This case-series reports the results of such peculiar ECMO configurations with PA cannulation in different clinical conditions.
\end{abstract}

Methods. All consecutive patients receiving PA cannulation (direct or percutaneous) from January 2015 to September 2018 in 3 Institutions were retrospectively reviewed. Isolated LV unloading or RV support, as well as dynamic support including initial drainage followed by perfusion through the PA cannula, were used as part of the ECMO configuration according to the type of patient and hemodynamic/functional needs.

Results. Fifteen patients ( 8 males, age range $45-73$ years, EuroSCORE log range $14.45 \%-91.60 \%$ ) affected by acute LV, RV, or bi-ventricular failure due to various etiologies, were supported by this ECMO mode. Percutaneous PA cannulation was performed in 10 patients, and direct in 5 cases. Dynamic ECMO management (initially draining and then perfusing through the PA cannula) was carried out in 6 patients. Mean ECMO duration was 9.1 days (range 6-17 days), one patient had pericardial fluid appearance during PA cannula implant (no lesion found at open-chest), and temporary circulatory support weaning was achieved in 14 patients (one transplanted). Three patients (20\%) died in-hospital, and 12 patients were successfully discharged without major complications.

Conclusion. Effective indirect LV unloading in peripheral V-A ECMO as well as isolated RV support can be achieved by PA cannulation. Such an ECMO configuration may allow to counteract common V-A ECMO shortcomings or allow dynamic/adjustable ECMO management according to peculiar ventricular dysfunction and hemodynamic needs. Percutaneous PA cannulation was shown to be safe and feasible without major complications. Additional investigation is needed to confirm the safety and efficacy of such an ECMO configuration and management in a larger patient population. 


\section{Introduction}

Veno-arterial extracorporeal membrane oxygenation (V-A ECMO) is an effective tool for cardio-circulatory assistance during acute and refractory cardiogenic shock [1]. This temporary circulatory support is used to counteract left ventricular (LV), right ventricular (RV), or bi-ventricular (BiV) dysfunction. However, despite well-established advantages related to restored adequate peripheral blood flow and oxygenation, $\mathrm{V}-\mathrm{A}$ ECMO may induce maladaptive hemodynamic changes. Indeed, due to ECMO-related retrograde flow towards the aortic valve, LV afterload is increased and this may induce de novo or further LV deterioration, with the worst scenario characterized by protracted aortic valve closure and LV blood stasis [2]. Several modalities have been proposed and used to address such complications with a wide range of direct or indirect LV unloading procedures, including enhanced right-sided drainage by means of an additional cannula positioned in the PA [2].

An additional potential drawback in peripheral $\mathrm{V}-\mathrm{A}$ ECMO is represented by a low oxygen saturation/provision in the upper part of the body due to de-oxygenated blood coming from the lung circulation to the LV and, hence, to the supra-aortic vessels, condition known as "Harlequin Syndrome" or "North/South Syndrome" [3].

Another important aspect of V-A ECMO relates to the use of such a temporary circulatory support in the presence of RV dysfunction. The prevalence of acute RV failure among acute heart failure-related hospital admissions ranges between $3 \%$ and $9 \%$ with an in-hospital mortality ranging from 5\% to $17 \%$ [4]. In cardiac surgery patients, regardless whether occurring pre-, intra-, or perioperatively, severe RV impairment has a prevalence below $10 \%$, but may be higher in patients undergoing heart transplant and left ventricular assist device therapy [5]. Complicated intensive care unit courses are commonly observed, and in-hospital mortality remains high $[5,6]$. Conventional peripheral V-A ECMO is a well-established support in the presence of isolated or associated RV failure, but negative impact on lung perfusion and LV performance may also occur with such an ECMO configuration [1-3].

Severe $\mathrm{BiV}$ failure in cardiac surgery patients represents an even more challenging condition. This deterioration is most probably due to overload conditions (long-lasting valve dysfunction), pre- or intraoperative myocardial ischemia (acute coronary stenosis or occlusion, inappropriate myocardial protection) or to severe inflammatory/immune response (acute myocarditis) $[7,8]$. The use of temporary circulatory support 
in this circumstance is usually challenging due to the different ventricular hemodynamics/behavior and recovery time during the illness course [9-17].

Based on the above-mentioned limitations and shortcomings with standard V-A ECMO configuration, it is clear that peripheral V-A ECMO may provide satisfactory peripheral blood flow and oxygenation, but induce maladaptive and untoward effects, either to the LV unloading or upper-body perfusion. It may also represent a too aggressive approach in case of isolated RV dysfunction. Furthermore, the application of static V-A ECMO configuration may not be ideal to address hemodynamic changes occurring during the illness course, or face isolated and individual ventricular response to temporary support. The possibility to design and apply modified and adjustable ECMO configurations which might address or avoid such complications, or favor a more adequate and hemodynamic-oriented support mode, would represent a more effective way to establish temporary support.

The use of pulmonary artery (PA) catheter or cannula has been previously reported to address LV unloading or RV support $[2,13]$. Nonetheless, this technique has been poorly investigated, besides limited case reports, as well as usually described as a fixed perfusion-related modality. We recently designed a modification of conventional ECMO modes with PA cannula [18], and applied this in patients with post-cardiotomy and nonpost-cardiotomy cardiogenic shock. The adopted strategy was meant to provide indirect LV unloading in case of V-A ECMO, but also to achieve an isolated RV or dynamic BiV hemodynamic support, taking advantage of the presence of a PA cannula in order to adjust the ECMO configuration and action in accordance to the ongoing cardiac function and recovery.

\section{Methods}

\section{Patients}

All the consecutive patients supported with V-A ECMO and adjunctive PA cannulation (direct or percutaneous) at Maastricht University Medical Center, Civic Hospital Brescia, and Sant'Orsola-Malpighi University Hospital Bologna, from January 2015 to September 2018 were retrospectively reviewed. The outcome of a modified ECMO configuration with PA cannulation for indirect LV unloading and its management in the presence of $\mathrm{RV}$ and $\mathrm{BiV}$ dysfunction were analyzed.

Definition of refractory LV, RV, or BiV dysfunction followed established criteria [19] in particular, in relation 
to acute and refractory RV failure, the presence of a dilated chamber (Figure 1), pre-operative severely increased right atrial pressure $(>16 \mathrm{~mm} \mathrm{Hg})$, dilated inferior vena cava $(>20 \mathrm{~mm})$ without inspiratory variation on transesophageal echocardiography, tricuspid annular plane systolic excursion $<12 \mathrm{~mm}$, with or without need of (intravenous) diuretic or inotropic support, were usual features. Signs of RV stasis (liver and kidney dysfunction confirmed by blood samples with increased related parameters) could also be present. Patients with ECMO implanted prior to surgery were excluded from this analysis.

The local ethical committee approved the current study design and waived the need for informed consent due to the observational character of the study (METC 2018-0797, November $7^{\text {th }} 2018$ ).

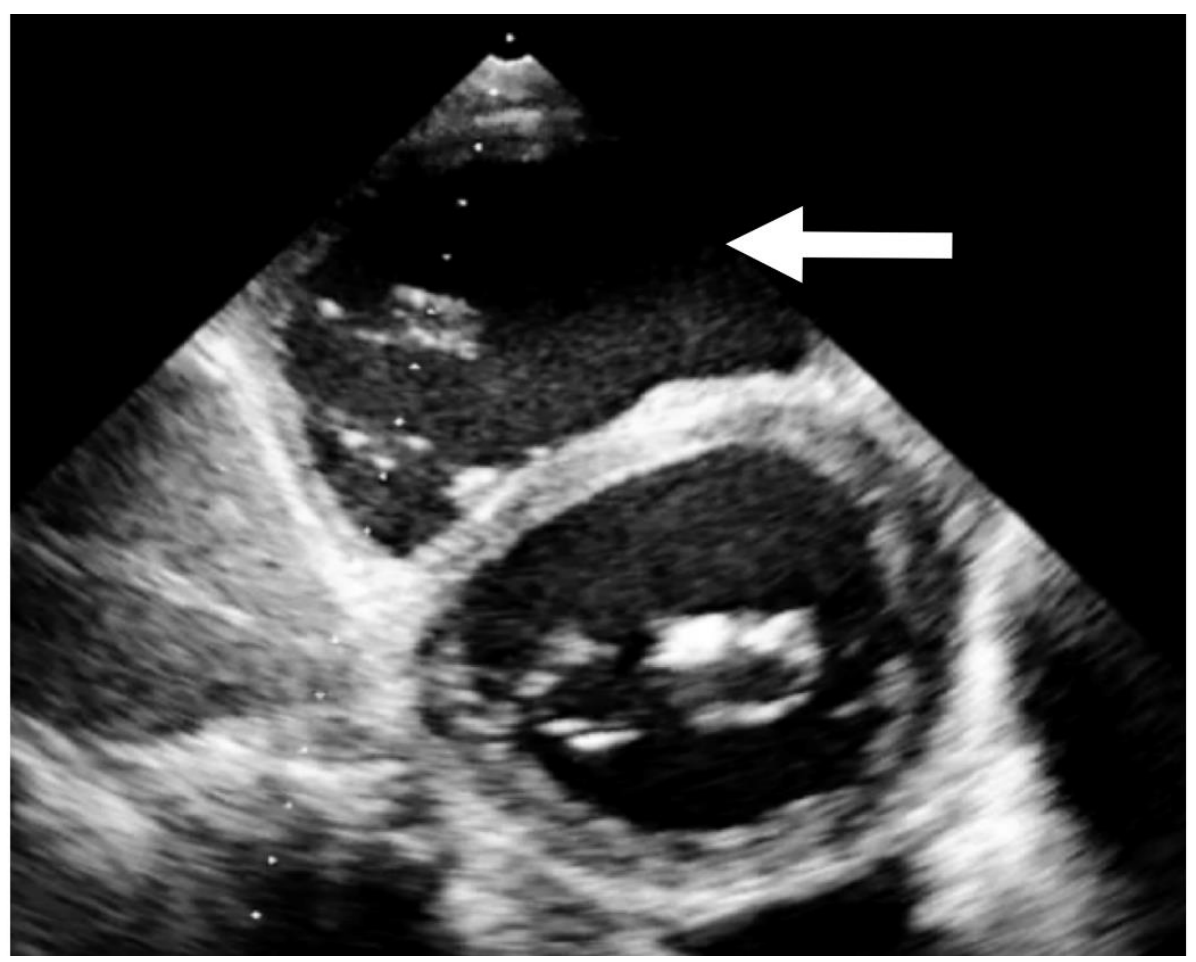

Figure 1. Preoperative transthoracic echo in a patient showing the marked bi-ventricular dilatation associated with severe dysfunction, particularly of the right side (white arrow).

\section{ECMO Configuration and Management}

Briefly, in case of V-A ECMO mode, the modified configuration included semi-percutaneous cannulation (cannula introduced in the vessels below the skin incision with Seldinger technique) of the femoral vein in the right atrium and femoral artery, with adjunctive PA cannulation. Configuration and different access possibilities are schematically presented in the Figure 2. The PA cannula is used, if needed, as an additional 
draining cannula to enhance LV unloading. The LV unloading was assessed by transesophageal echocardiogram, in relation to LV distension/dimension, as well as by looking at the signs of blood stasis and left cardiac valve openings (aortic and mitral). The aortic pressure curve pulsatility (presence and index) was used to alert attending personnel about the urgent need for echo assessment, besides routine and periodical echocardiographic monitoring. Indications for LV venting have been previously presented [2, 17].

The same PA cannula might provide blood flow into the PA in case of RV bypass mode or hybrid V-A ECMO [18]. In one case, the right atrium was cannulated through the femoral vein as inlet arm and the PA cannula as outlet arm, enabling isolated RV support. Cannulation of PA was carried out either directly (intra-operatively) (Figure 3A) or percutaneously (Figure 3B) according to the timing and clinical situations warranting the temporary circulatory support.

V-A ECMO weaning protocol was performed according to the standard ELSO guidelines (https://www.elso.org/Resources/Guidelines.aspx). Right ventricular free wall myocardial recovery and a tricuspid annular plane systolic excursion greater than $12 \mathrm{~mm}$ at echo and an arterial and venous saturation greater than $90 \%$ and $65 \%$ respectively, were used as indicators for a reasonable weaning off of PA cannulation.

\section{CONFIGURATION}

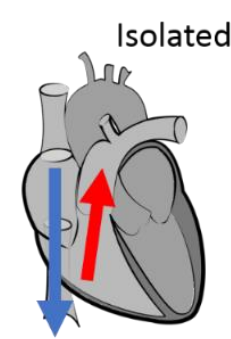

Oxy-RVAD

(with oxygenator)

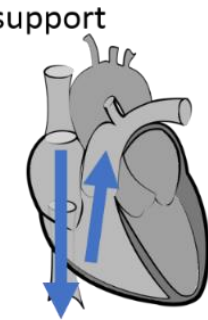

Mini-RVAD

(without oxygenator)

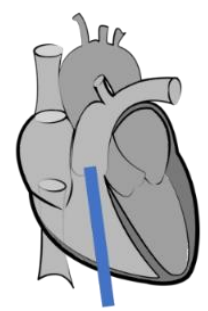

Direct

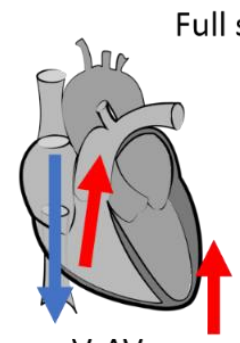

V-AV

ACCESS

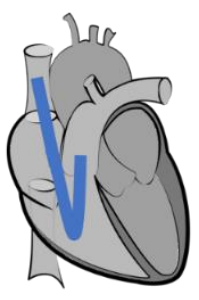

Percutaneous

Figure 2. Schematic overview of configuration and access possibilities with adjunctive pulmonary artery cannulation. 
Oxy-RVAD: oxygenated right ventricular assist device, mini-RVAD: minimally invasive right ventricular assist device, $V$-AV: veno-arterial venous, $V V$-A: venovenous-arterial.

\section{Pulmonary artery cannulation technique}

For direct PA cannulation, carried out in operating theater during full or partial sternotomy operations, two 4/0 polypropylene purse-string sutures reinforced with Teflon pledgets felts are placed on the anterior wall of the PA, $2 \mathrm{~cm}$ downstream the pulmonary valve and root. A 19 or 21 French (according to the patients' body size) cannula with a multi-hole tip (Byomedicus ${ }^{\circledR}$, Medtronic, MN) is then introduced in the PA and tunneled at the subxyphoid space, and connected to the ECMO circuit. As an alternative method for direct cannulation, a vascular prosthesis is anastomosed to the main PA using a two 5/0 polypropylene running suture and tunneled at the subxyphoid space, allowing postoperative cannula withdrawal and prosthesis ligation without reopening the sternum [20]. The cannula is connected to the outflow or inflow of the ECMO circuits according to the required ECMO configuration and modality, including a veno-arterial venous mode, with the PA cannula as an additional central perfusion port and connected to the outflow (arterial) circuit, or a venovenous-arterial mode, with the PA cannula as an adjunctive central draining cannula and connected to the inflow (venous) circuit.

When PA cannulation is warranted post-operatively or not in surgical candidates, a percutaneous cannulation is advocate under fluoroscopy and echocardiographic guidance [18]. A right internal jugular venous access is established using direct ultrasound visualization. A soft guidewire is placed in the PA with fluoroscopic confirmation or using a Swan-Ganz catheter. Then, along the guidewire, a pig-tail catheter is positioned in the same location of the guidewire tip, and, in case of doubtful position, transesophageal echocardiography is used or a small dye injection is delivered to confirm the relationship between the pulmonary valve and the guidewire tip. After withdrawal of the soft guidewire, a superstiff guidewire (Amplatzer, Boston Scientific, Malborough, MA) is advanced within the pig-tail catheter which is finally removed. Through a stepwise dilatation of the skin access, a 19- or 21-French (according to the patient's body size) multi-hole tip cannula (Biomedicus ${ }^{\circledR}$, Medtronic, Minneapolis, MN) as part of a double-cannulation configuration (a venous cannula positioned in the right atrium from the right or left femoral vein), or (29 French ProtekDuo® LivaNova, London, UK) a double-lumen cannula, in the single-cannula configuration, is finally positioned along the superstiff guidewire 
in the main pulmonary trunk, after which fluoroscopic and transesophageal assessment can confirm the adequate position (Figure 3B).
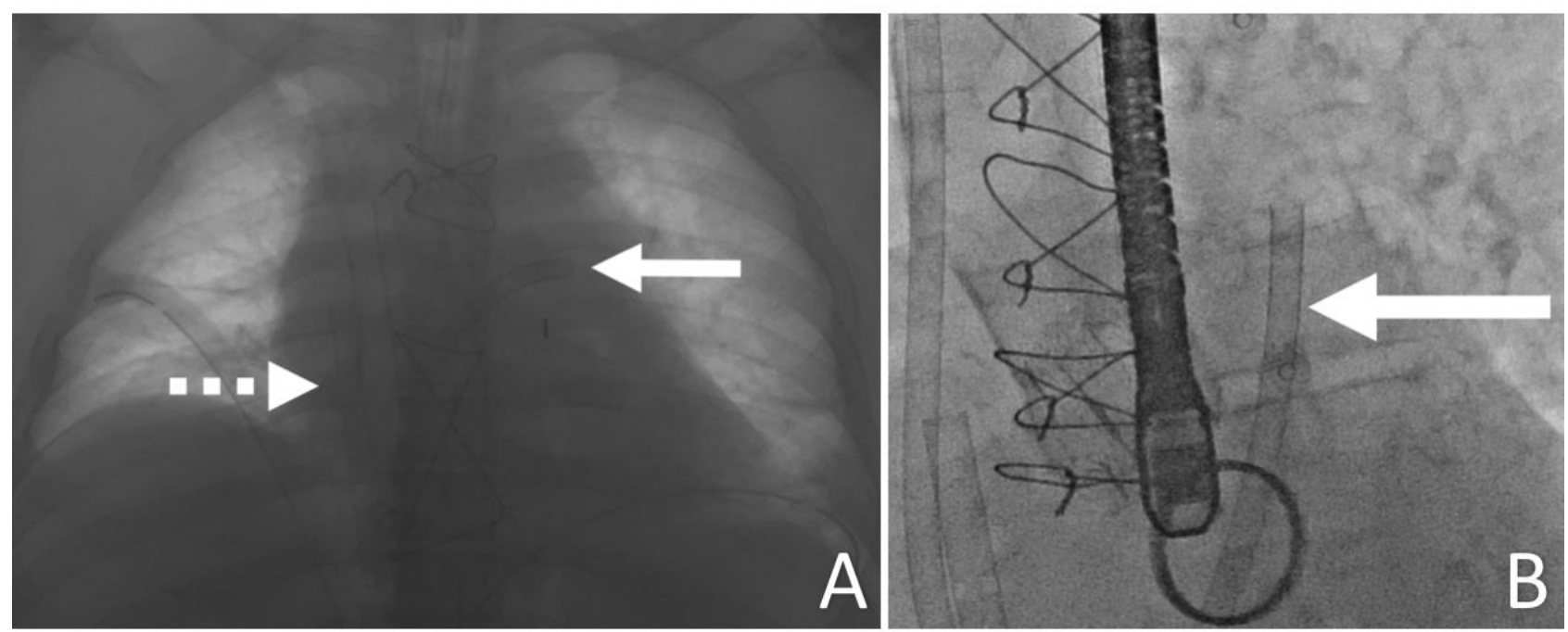

Figure 3. A. X-ray showing a cannula positioned in the main pulmonary artery with direct cannulation (uninterrupted arrow) and a cannula in the right atrium (dotted arrow), B. Fluoroscopy picture showing the percutaneous cannula positioned in the main pulmonary artery from the right internal jugular vein

\section{Results}

Fifteen patients were submitted to PA cannulation during ECMO support for LV, RV or BiV failure. Demographics, operative characteristics, ECMO indication and configuration, and additional assist devices are presented in Table 1. Age ranged from 45 to 73 years, 8 patients (53\%) were male. The etiology of cardiogenic shock included post-cardiotomy ( 9 patients), acute myocardial infarction ( 3 patients), acute myocarditis ( 2 patients) and decompensated dilated cardiomyopathy (1 patient). All patients had severe RV dysfunction (tricuspid annular plane systolic excursion ranging from 4 to $9 \mathrm{~mm}$ prior to surgery) and dilatation (Figure 1). All subjects had associated LV dysfunction (LV ejection fraction ranged from $14 \%$ to $35 \%$, mean $20 \%$ ). Twelve patients had concomitant intra-aortic balloon pump ( 2 already present at the time of surgery). Ten patients received percutaneous cannulation of the PA, whereas 5 subjects had direct cannulation of the main 
trunk of the PA intraoperatively. The duration of ECMO ranged from 5 to 17 days, and length of intensive care unit-stay from 15 to 40 days.

No major complications were observed in relation to PA cannulation, either direct or percutaneous, achieving an optimal cannula position and flow characteristics in all cases (Table 2). Pericardial effusion during percutaneous cannulation of the PA, requiring an open-chest procedure due to the suspicion of RV or right atrial perforation, but no injury was found, with final direct PA cannulation. In one patient, with isolated RV failure, the PA cannula was used as a reperfusion cannula from the beginning of temporary support.

Major in-hospital complications are listed in Table 2. In-hospital mortality rate was $20 \%$, including one death on-ECMO due to massive cerebral hemorrhage, one for septic shock following a successful weaning, and one due to sepsis secondary to leg ischemia. Eventually, 12 patients were discharged, and are alive as well as in good clinical conditions at follow-up (range 6 months to 30 months).

Table 1. Patient charachteristics. 


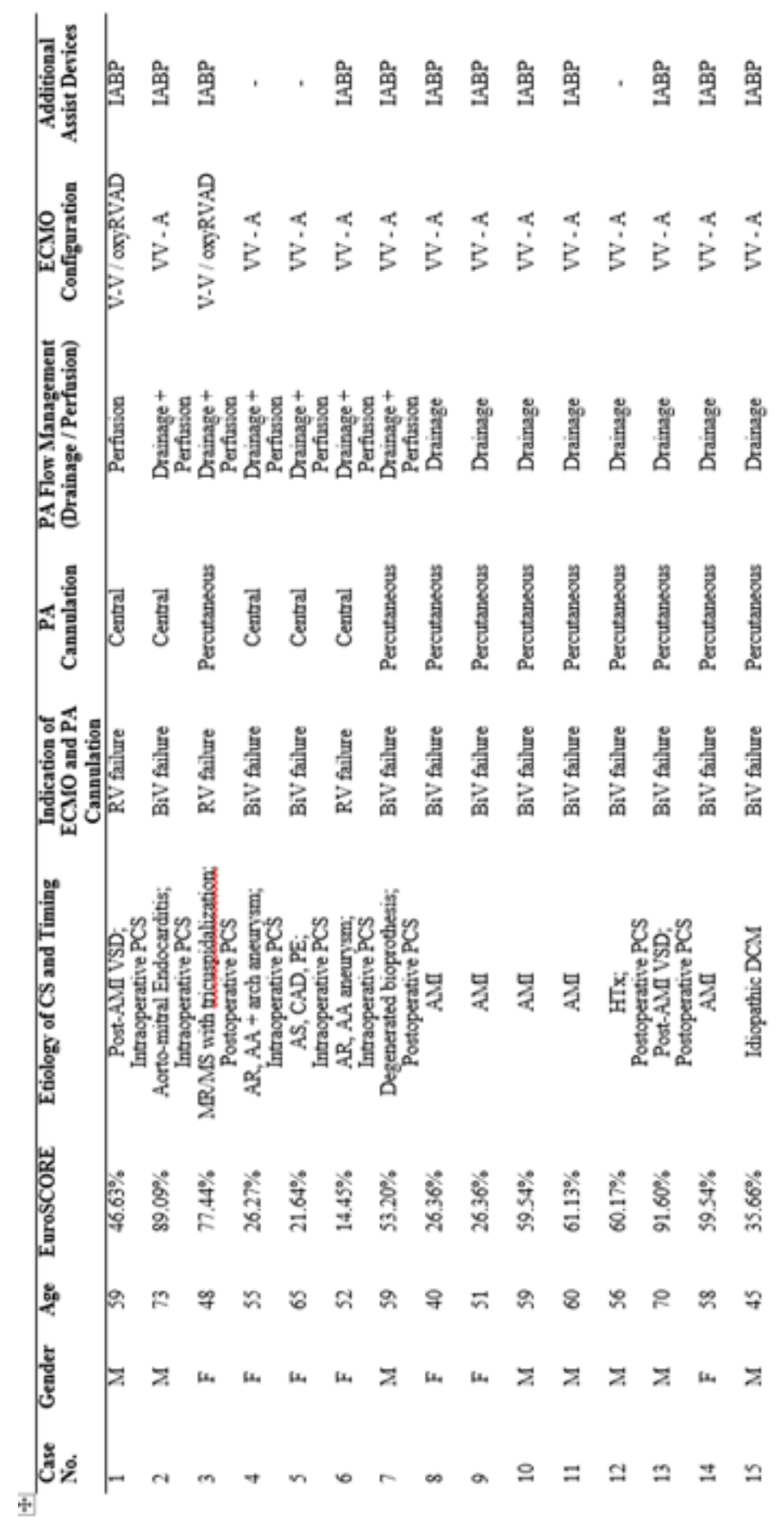

EuroSCORE: European System for Cardiac Operative Risk Evaluation, ECMO: Extracorporeal Membrane Oxygenation, PA: Pulmonary Artery, M: Male, F: Female, AMI: Acute Myocardial Infarction, VSD: ventricular septal defect; PCS: post-cardiotomy shock; RV: Right Ventricle, V-V: Veno-Venous, oxyRVAD: oxygenated Right Ventricular Assist Device, IABP: Intra-Aortic Balloon Pump, MR: Mitral Regurgitation, MS: Mitral Stenosis, AR: Aortic Regurgitation, AA: Ascending Aorta, BiV: Biventricular, VV-A: Veno-Venous Arterial, AS: Aortic Stenosis, CAD: Coronary Artery Disease, PE: Pulmonary Embolism, AM: Acute Myocarditis, HTx: Heart transplantation, DCM: Dilative Cardiomyopathy 
Table 2: PA-related flow and outcomes

\begin{tabular}{|c|c|c|c|c|c|c|c|}
\hline Case no. & $\begin{array}{l}\text { PA cannula } \\
\text { flow ( }(1 / \mathrm{min})\end{array}$ & $\begin{array}{l}\text { ECMO duration } \\
\text { (days) }\end{array}$ & $\begin{array}{l}\text { Length of } \\
\text { ICU stay (days) }\end{array}$ & Complications & ECMO weaning & Cardiac recovery & Outcome \\
\hline 1 & NA & 5 & 20 & & Yes & Full & Discharged \\
\hline 2 & 2.1 & 9 & 28 & & Yes & Full & Discharged \\
\hline 3 & 1.7 & 6 & 15 & & Yes & Full & Discharged \\
\hline 4 & 1.6 & 13 & 37 & Renal failure, iCVA, sepsis & Yes & Full & Discharged \\
\hline 5 & 1.8 & 8 & 40 & DSWI & Yes & Partial & Discharged \\
\hline 6 & 1.7 & 17 & 17 & Cerebral haemorrhage & Yes & Partial & Died \\
\hline 7 & 1.1 & 11 & 19 & Leg amputation & Yes & Partial & Died \\
\hline 8 & 1.6 & 8 & 20 & Cerebral haemorrhage & Yes & Full & Discharged \\
\hline 9 & 1.6 & 7 & 21 & Leg ischaemia, renal failure & Yes & Full & Discharged \\
\hline 10 & 1.8 & 10 & 25 & Cerebral haemorrhage, renal failure & Yes & Full & Discharged \\
\hline 11 & 1.7 & 9 & 36 & Renal failure & No & Transplant & Discharged \\
\hline 12 & 1.8 & 6 & 28 & Renal failure, pneumonia & Yes & Full & Discharged \\
\hline 13 & 1.7 & 10 & 26 & Pericardial effusion & Yes & Full & Discharged \\
\hline 14 & 1.6 & 10 & 19 & Leg ischaemia, iCVA & Yes & Full & Died \\
\hline 15 & 1.7 & 8 & 40 & Renal failure, pneumonia & Yes & Full & Discharged \\
\hline
\end{tabular}

Flow data are provided as peak values achieved; perfusion flows were at the time controlled by a Hoffman clamp to avoid hyperperfusion during venoarterial-venous ECMO.

DSWI: deep sternal wound infection; ECMO: extracorporeal membrane oxygenation; ICU: intensive care unit; iCVA: ischaemic cerebrovascular accident; NA: not applicable; PA: pulmonary artery.

\section{Discussion}

The presence of severe $\mathrm{RV}$ or $\mathrm{BiV}$ dysfunction, regardless of the timing of onset and duration, is a well-known negative determinant of unfavorable in-hospital outcome in cardiac surgery patients [4,7-9]. In case of refractory and persistent $\mathrm{RV}$ or $\mathrm{BiV}$ failure, the use of mechanical circulatory support devices may represent a favorable option. This method facilitates hemodynamic stabilization, limits dosages of inotropic or vasopressors, and allows sternal closure. The use of temporary circulatory support systems is usually applied in these circumstances, as more complex and long-lasting mechanical devices are more expensive, more cumbersome to implant, and mostly available in transplant-centres. V-A ECMO is currently the most widely adopted system used for temporary assistance in post-cardiotomy and post-acute myocardial infarction adult patients, although other types of devices have been used successfully $[6,7,15,16]$. The conventional ECMO configuration is $\mathrm{V}-\mathrm{A}$, including right atrial drainage, via direct cannulation or through the femoral vein, and reinfusion of oxygenated blood into a peripheral arterial vessel (femoral or axillary artery, peripheral access) or directly into the ascending aorta (central approach). The conventional V-A ECMO mode has obvious hemodynamic advantages (RV unloading, peripheral oxygenation, temperature control), but carries several disadvantages. For example, patients may present with an increased LV afterload leading to insufficient LV unloading and upper-body hypoxemia.

Our series presents the adjunct of a cannula into the PA, acting alternatively as additional drainage or perfusion cannula. As demonstrated previously, the addition of a PA cannula as a drainage arm of the ECMO circuit is 
effective in increasing the venous return and acts as an indirect LV unloading configuration [2]. Indications and potential advantages of PA cannulation are reported in Table 3. As a perfusion cannula, the PA approach has been used in several ECMO or RV support configurations $[15,16]$.

\section{Similar techniques or cannulas}

Pulmonary artery cannulation is not new in ECMO management. Kolobow et al. already described this technique for veno-venous ECMO almost 3 decades ago [21]. Avalli and coworkers used percutaneous PA cannulation for increasing LV unloading [22]. Recently, Napp and colleagues reported a first-in-man case of a fully percutaneous cardiac assistance using the right atrium/PA approach for RV bypass and a transaortic device for LV support [23]. The use of PA cannulation to achieve a more effective LV venting is also not new in routine cardiac surgery or other ECMO modes [24-25]. Such a venting method may not be as effective as direct LV unloading, but provides sufficient adjunctive drainage to right atrial cannula avoiding the need for left cardiac chamber-related access or procedures (right superior pulmonary vein cannulation, septostomy or intra-septal or cardiac apex cannulation). Indeed, left-sided venting approach is not void of severe or even fatal complications [26, 27].

In this study, we presented the results of the proposed adoption of a PA-related cannulation with different approaches, including a percutaneous implant [23]. Percutaneous approach may take from 20 to 30 minutes, but with a Swan-Ganz catheter already in place, implant may take even less [18]. Although percutaneous cannulation is usually performed pre- or postoperatively, this method may be applied in the operating room, if a hybrid theatre is available [18]. Such a cannulation strategy might be part of the surgical preparation and used during cardiopulmonary bypass in high-risk surgical case for perioperative RV dysfunction, and then switched to the ECMO circuit once the operation is concluded. This ECMO approach, in combination with femoral vein cannulation, facilitates closing of the sternum and may subsequently avoid the need of chest reopening at the time of explantation of the cannula, potentially reducing open-chest related complications [28]. However, other minimally invasive approach to interrupt the PA perfusion have been recently proposed, like the use of PA cannulation through a prosthesis [29]. We used direct cannulation of the PA in cardiac surgery patients in order to reduce the operating time and risk of bleeding or injury of the PA, but we acknowledge the advantage of the prosthesis-based technique to allow a closed-chest decannulation [28]. 
Finally, based on the intrinsic structural differences between the LV and RV, recovery of the left chamber usually occurs more quickly than the right chamber. Therefore, the presence of PA cannulation may be used first as a dual-section drainage (right atrium and PA, therefore venovenous-arterial mode). Then, once the LV has shown some sign of recovery, or if an upper-body deoxygenation is present (Harlequin Syndrome), a venoarterialvenous configuration may be established without adding other cannulas, but only changing flow direction in the PA cannula, as presented in our case series. As shown, adverse events might be low. Although not observed in our study cohort, PA cannulation could present some peculiar complications, like RV or PA injury or perforation with subsequent pericardial effusion and tamponade, as well as it may induce ventricular arrhythmias during guidewire or cannula implantation. Although in our experience we observed pericardial effusion only in one patient, without any observation of RV or PA perforation after chest opening, these events should be taken into consideration.

Renal failure is associated with poorer prognosis in patients receiving ECMO [30], and this adverse event was the most common complication observed in our patient cohort in accordance to the ELSO International Registry data). In our experience, since the percutaneous PA cannulation is mainly performed under fluoroscopy and transesophageal echo, no correlation can be found between acute renal failure and contrast dye administration.

This study has the obvious limitations of a retrospective observational study. Although beyond the scope of this study, the lack of control group and hemodynamic data to compare and to assess the efficacy of PA cannulation in LV unloading during V-A ECMO support with other conventional decompression techniques, represent major limitations. Finally, the use of PA cannulation in ECMO and RV support management warrants further investigation to fully elucidate implant-related complications and efficacy, either in terms of drainage or perfusion mode.

Although our cohort consists of 15 patients, it is the largest multicenter case series to report on such an ECMO configuration until now. Combining PA cannulation with usual inferior or superior vena cava/right atrial cannulation provides possibility for both LV unloading as well as RV assist bypassing the right ventricle. The initial drainage and then perfusion of the PA in the same patient represents a novel technique. Further investigation and research are still required to conclusively confirm the benefits of adjunctive PA cannulation 
in V-A ECMO patients. However, safety and efficacy of PA cannulation was demonstrated in a very high-risk patient, affected by severe RV or BiV dysfunction.

In conclusion, adjunctive PA cannulation, whenever preoperative or intraoperative $\mathrm{BiV}$ or $\mathrm{RV}$ dysfunction occurs, may allow a better patient management, allowing additional RV drainage and LV unloading, or perform RV support, according to the patient hemodynamic needs.

Table 3: Indications and advantages of pulmonary artery cannulation for VA ECMO or RV support

Drainage

Indirect LV unloading (poor LV function with LV or LA stasis/acute pulmonary oedema/LV distension)

Pulmonary haemorrhage

Biventricular failure

Acute pulmonary hypertension

Perfusion

Harlequ in (or North/South) syndrome in VA ECMO

Isolated RV failure

Acute pulmonary embolism

Biventricular failure

Pulmonary contusion

W ECMO with RV failure

W ECMO with high recirculation

ECMO: extracorporeal membrane oxygenation; LA: left atrial; LV: left ventricular; RV: right ventricular; VA: venoarterial; WV: venovenous.

\section{References}


[1] Bellumkonda L, Gul B, Masri SC. Evolving concepts in diagnosis and management of cardiogenic shock. Am J Cardiol 2018;122:1104-10

[2] Meani P, Gelsomino S, Natour E, Johnson DM, Rocca HB, Pappalardo F, et al. Modalities and effects of left ventricular unloading on extracorporeal life support: a review of the literature. Eur J Heart Fal 2017;19:81-88

[3] Lo Coco V, Lorusso R, Raffa GM, Malvindi PG, Pilato M, Martucci G, et al. Clinical complications during veno-arterial extracorporeal membrane oxigenation in post-cardiotomy and non post-cardiotomy shock: still the achille's heel. J Thorac Dis. 2018;10:6993-7004

[4] Harjola VP, Mebazaa A, Celutkiene J, Bettex D, Bueno H, Chioncel O et al. Contemporary management of acute right ventricular failure: a statement from the Heart Failure Association and the Working Group on Pulmonary Circulation and Right Ventricular Function of the European Society of Cardiology. Eur J Heart Fail 2016;18:226-41

[5] Kaul TK, Fields BL. Postoperative acute refractory right ventricular failure: incidence, pathogenesis, management and prognosis. Cardiovasc Surg 2000;8:1-9

[6] Itagaki S, Hosseinian L, Varghese R. Right ventricular failure after cardiac surgery: management strategies. Semin Thorac Cardiovasc Surg 2012;24:188-94

[7] Lomivorotov VV, Efremov SM, Kirov MY, Fominskiy EV, Karaskov AM. Low-CardiacOutput Syndrome After Cardiac Surgery. J Cardiothorac Vasc Anesth 2017;31:291-308

[8] Cholley B, Caruba T, Grosjean S, Amour J, Ouattara A, Villacorta J et al. Effect of Levosimendan on Low Cardiac Output Syndrome in Patients With Low Ejection Fraction Undergoing Coronary Artery Bypass Grafting With Cardiopulmonary Bypass: The LICORN Randomized Clinical Trial. JAMA 2017;318:548-56 
[9] Kapur NK, Esposito ML, Bader Y, Morine KJ, Kiernan MS, Pham DT et al. Mechanical Circulatory Support Devices for Acute Right Ventricular Failure. Circulation 2017;136:314-26

[10] Haneya A, Philipp A, Puehler T, Rupprecht L, Kobuch R, Hilker M et al. Temporary percutaneous right ventricular support using a centrifugal pump in patients with postoperative acute refractory right ventricular failure after left ventricular assist device implantation. Eur J Cardiothorac Surg 2012;41:219-23

[11] Liu KS, Tsai FC, Huang YK, Wu MY, Chang YS, Chu JJ et al. Extracorporeal life support: a simple and effective weapon for postcardiotomy right ventricular failure. Artif Organs 2009;33:5048

[12] Lang SA, O'Neill B, Waterworth P, Bilal H. Can the temporary use of right ventricular assist devices bridge patients with acute right ventricular failure after cardiac surgery to recovery? Interact Cardiovasc Thorac Surg 2014;18:499-510

[13] Giesler GM, Gomez JS, Letsou G, Vooletich M, Smalling RW. Initial report of percutaneous right ventricular assist for right ventricular shock secondary to right ventricular infarction. Catheter Cardiovasc Interv 2006;68:263-6

[14] Kretzschmar D, Lauten A, Ferrari MW. In vitro evaluation of a novel pulsatile right heart assist device - the PERKAT system. Int J Artif Organs 2015;38:537-41

[15] Kapur NK, Paruchuri V, Korabathina R, Al-Mohammdi R, Mudd JO, Prutkin J et al. Effects of a percutaneous mechanical circulatory support device for medically refractory right ventricular failure. J Heart Lung Transplant 2011;30:1360-7

[16] Takayama H, Naka Y, Kodali SK, Vincent JA, Addonizio LJ, Jorde UP et al. A novel approach to percutaneous right-ventricular mechanical support. Eur J Cardiothorac Surg 2012;41:423-6 
[17] Meani P, Delnoij T, Raffa GM, Morici N, Viola G, Sacco A, et al. Protracted aortic valve closure during peripheral veno-arterial extracorporeal life support: is intra-aortic balloon pump an effective solution? Perfusion. 2019;34:35-41.

[18] Loforte A, Baiocchi M, Gliozzi G, Coppola G, Di Bartolomeo R, Lorusso R. Percutaneous pulmonary artery venting via jugular vein while on peripheral extracorporeal membrane oxygenation running: a less invasive approach to provide full biventricular unloading. Ann Cardiothorac Surg. 2019;8:163-166

[19] Ponikowski P, Voors AA, Anker SD, Bueno H, Cleland JGF, Coats AJS, et al. 2016 ESC Guidelines for the diagnosis and treatment of acute and chronic heart failure: The Task Force for the diagnosis and treatment of acute and chronic heart failure of the European Society of Cardiology (ESC)Developed with the special contribution of the Heart Failure Association (HFA) of the ESC. Eur Heart J. 2016;37:2129-2200

[20] Lorusso R, Bidar E, Natour E, Heuts S. Minimally invasive management of central ECMO after ascending aortic surgery. J Card Surg 2019;34:131-33

[21] Kolobow T, Rossi F, Borelli M, Foti G. Long-term closed chest partial and total cardiopulmonary bypass by peripheral cannulation for severe right and/or left ventricular failure, including ventricular fibrillation. The use of a percutaneous spring in the pulmonary artery position to decompress the left heart. ASAIO Trans 1988;34:485-9

[22] Avalli L, Maggioni E, Sangalli F, Favini G, Formica F, Fumagalli R. Percutaneous left-heart decompression during extracorporeal membrane oxygenation: an alternative to surgical and transeptal venting in adult patients. ASAIO J 2011;57:38-40

[23] Napp LC, Vogel-Claussen J, Schafer A, Haverich A, Bauersachs J, Kuhn C et al. First-in-Man Fully Percutaneous Complete Bypass of Heart and Lung. JACC Cardiovasc Interv 2017;10:e231-e33 
[24] Mitchell IM, Prabhakar G, Maughan J, Taylor DN. Pulmonary artery versus left ventricular venting: a radioisotope study of left ventricular function. Ann Thorac Surg 1989;48:699-703

[25] Kimura M, Kinoshita O, Fujimoto Y, Murakami A, Shindo T, Kashiwa K et al. Central extracorporeal membrane oxygenation requiring pulmonary arterial venting after near-drowning. Am J Emerg Med 2014;32:197 e1-2

[26] Hong JH, Choi JW, Moon JH, Lim SH. Pseudoaneurysm of left ventricular apex caused by a left ventricular venting catheter through the right superior pulmonary vein. J Thorac Cardiovasc Surg 2007;134:229-30

[27] Kao CL, Chang JP. Left ventricular pseudoaneurysm secondary to left ventricular apical venting. Tex Heart Inst J 2003;30:162-3

[28] Raffa GM, Kowalewski M, Brodie D, Ogino M, Whitman G, Meani P et al. Meta-Analysis of Peripheral or Central Extracorporeal Membrane Oxygenation in Postcardiotomy and NonPostcardiotomy Shock. Ann Thorac Surg. 2019;107:311-321

[29] Saeed D, Maxhera B, Kamiya H, Lichtenberg A, Albert A. Alternative right ventricular assist device implantation technique for patients with perioperative right ventricular failure. $\mathrm{J}$ Thorac Cardiovasc Surg 2015;149:927-32

[30] Yan X, Jia S, Meng X, Dong P, Jia M, Wan J at al. Acute kidney injury in adult postcardiotomy patients with extracorporeal membrane oxygenation: evaluation of the RIFLE classification and the Acute Kidney Injury Network criteria. Eur J Cardiothorac Surg. 2010;37:334-8 
CHAPTER 9

\section{GENERAL DISCUSSION}


ECMO-related technology has witnessed a remarkable progress in the last 20 years. More advanced, user friendly, miniaturized technology has made a wider application of such a temporary support possible, particularly in several instances once viewed as absolute contraindications. ${ }^{1,2}$ In the face of its recent exponential increase, we must question in which patients is it truly beneficial, giving patients time to recover rather than simply prolonging the time of death. ECMO enthusiasts are particularly vulnerable to criticism as overall survival for PC-ECMO is not improving. ${ }^{3}$ Its growth, as has been alluded to, is related to easier ECMO institution, more candidates due to increased severity of patient disease, more complex procedures with acute, post bypass myocardial dysfunction, and the surprising salvage of some patients who otherwise would never have survived. ${ }^{4-12}$ Reoperations, advanced age, surgical urgency, increasingly poor cardiac reserve are increasingly present in cardiac surgery candidates, all contributing to higher risk for cardiopulmonary insufficiency after CPB weaning, with subsequent pressure on the caregiving team to institute PC-ECMO to avoid ominous intra-operative outcome. As a consequence, intraoperative death is nowadays a rare event. These circumstances, however, increase the likelihood that ECMO is being over-utilized, in an effort to avoid intraoperative mortality, therefore, moving the inevitable event from the operating room to the ICU. ${ }^{1,10}$ ECMO over-utilization needs to be recognized and managed, to maximize survival, minimizing its futile use. Obvious targets to accomplish this include, improvements in technological aspects of the circuitry, e.g the pump, oxygenator, and tubing design, more reliable anticoagulation, rational vasoactive/inotropic support, a better understanding of the most effective ways to achieve temporary cardiopulmonary support, more effective monitoring, and improving care-giver education and training.

If PC-ECMO is currently considered a must for any cardiac surgery center in order to provide a liferescue tool in case of need, it is also obvious that inexperienced surgeon or surgical- and ICU teams may face intra- or perioperative conditions which may require such a temporary support. However, even where expertise and advanced ECMO programs exist, PC-ECMO application may still face questionable and controversial issues, like proper patient selection, optimal ECMO configuration, 
adequate prevention or management of complications, and appropriate decision making for recovery or switch to more advanced therapies. How could these approaches be achieved allowing the attending personnel to appropriately manage PC-ECMO cases? How to avoid futile or useless and high resource-consumption procedure to be implemented, either in inexperienced or experienced centers?

The optimal understanding of ECMO application, particularly in the PC setting, remains of paramount importance to have a comprehensive overview of the actual impact and advantages/disadvantages of such a temporary support.

The assessment of single-centre experiences in overall PC scenarios, or specific niches, like HTx or interventional procedures, may provide additional and relevant information about the potentials and, most likely, the future direction of ECMO use and further development.

Thanks to several individual personal experiences, as presented in the patients series on ECMO in PC after HTx or general cardiac surgery, or the appraisal of the further new options/potentials of the new ECMO configuration, as demonstrated in our experience with the cannulation of the PA, underline once again the real contribution ECMO may provide in these complex situations. Indeed, by thorough investigation regarding the use of PC-ECMO in adult and pediatric patients, we could highlight the dark and shining aspects of ECMO along the time and, particularly, how variable are the individual experiences in such a setting.

The application of ECMO as a rescue therapy/support, in the presence of perioperative shock like in HTx or general cardiac surgery, has shown the potential benefits, but also the high rate of complications, somehow apparently strictly related to the extent of maladaptive body reaction, secondary to the interaction between the body and a prolonged extracorporeal circulation, but also to intrinsic potential shortcoming related to the invasiveness of the technique.

Our data could once again demonstrate how some complications (like PC bleeding) may affect PCECMO, but they may be related to the type of ECMO configuration and approach. 
Despite the well-known or still undetermined shortcomings related to the ECMO, or in particular to PC-ECMO, it is increasingly clear that such a temporary support allowed the rescue and temporary support to bridge the patient to organ recovery or to more advanced care, in situation in which any further or different option would have been inapplicable with inevitable patient death.

The use of ECMO as a transient circulatory or respiratory, or concomitant assistance of both impaired systems, has been shown to represent an invaluable opportunity, although characterized by several drawbacks.

The acquisition of new concepts and modalities of support, as we were able to demonstrate through the application of cannulas in the pulmonary artery via direct or percutaneous approach, have open the way to enhanced modalities and possibilities of further and different type of cardio-circulatory support. The availability of this system may enhance the approaches for interventional cardiology (like protected coronary angioplasty or transcatheter valve implantation), but also represent a currently necessary tool to address refractory compromise of the cardio-circulatory function.

The advancement of four fields could improve the effectiveness of PC-ECMO: 1) underlying pathophysiology of cardiopulmonary failure , 2) the pathophysiologic consequences of ECMO on the subject, 3) a standardized, evidence based, structured approach to monitoring, 4) developing better biomarkers as predictors, and management guide, 5) ethical and economics implications of ECMO utilization, and 6) provider training and education. As medicine approaches these 6 aspects of ECMO, assuredly, the current picture of "ECMO" will metamorphose in the years to come.

Finally, ethics as well as resource allocation are critical factors to be considered when employing ECMO as therapy. ${ }^{3}$ Perhaps as a result of our lack of a sophisticated understanding of its true indications, appropriate patient targets, its management, and its exit strategies, the significant associated costs and ethical dilemmas seem ever present and at times, unjustifiable. For sure, the dilemmas associated with ECMO are all part of a complex scenario, but, to a large degree, providers must accept responsibility for shepherding its use, despite the lack of data to inform them. ${ }^{1,3}$ The exponential growth in ECMO use will soon be linked to ethical dilemma related to resource allocation 
and expenditures. The question already being asked is whether to provide unconditional access to ECMO, and particularly to PC-ECMO. Should ECMO program be restricted to centralized hub centers, or should hospital case-volumes be subjected to limitations? Limiting access to PC-ECMO to only in few expert centers is already untenable, as the need is high, and its initiation is not complicated. It has been said recently that great power calls for great responsibility ${ }^{3}$ : PC- ECMO is a perfect target for this statement. Therefore, additional efforts aimed at the above 6 target areas is mandatory, such that, hopefully, in the near future, the rationale for its use will be understood and justifiable in all cases. 
1. Sylvin EA, Stern DR, Goldstein DJ. Mechanical support for postcardiotomy cardiogenic shock: has progress been made? J Card Surg 2010;25:442-54

2. Stretch R, Sauer CM, Yuh DD, Bonde P. National trends in the utilization of short-term mechanical circulatory support. J Am Coll Cardiol 2014;64:1407-15

3. Whitman GJR. Extracorporeal membrane oxygenation for the treatment of postcardiotomy shock. J Thorac Cardiovasc Surg 2016;153:95-101

4. Saxena P, Neal J, Joyce LD, et al. Extracorporeal membrane oxygenation support in postcardiotomy elderly patients: the Mayo Clinic experience. Ann Thorac Surg 2015;99:2053-60

5. Ranucci M, Barushnikova E, Isgrò G, et al. Heparin-like effect in post-cardiotomy extracorporeal membrane oxygenation patients. Critical Care 2014;18:504-10

6. Smith C, Bellomo R, Raman JS, et al. An extracorporeal membrane oxygenation-based approach to cardiogenic shock in an older population. Ann Thorac Surg 2001;71:1421-7

7. Tarzia V, Bertolussi G, Bianco R, et al. Extracorporeal life support in cardiogenic shock: impacts of acute versus chronic etiology on outcome. J Thorac Cardiovasc Surg 2015;150:333-40

8. Wong JK, Smith TN, Pitcher HT, Hirose H, Cavarocchi NC. Cerebral and lower limb near infra-red spectroscopy in adults on extracorporeal membrane oxygenation. Artif Organs. 2012;36:659-67

9. Sauer CM, Yuh DD, Bonde P. Extracorporeal membrane oxygenation use increased $433 \%$ in adults in the United States from 2006 to 2011. ASAIO J 2015:61:31-36

10. McCarthy FH, McDermott KM, Kini V, et al. Trends in U.S. extracorporeal membrane oxygenation use and outcome: 2002-2012. Sem Thorac Surg 2015;27:81-88

11. Curtis JJ, McKenney-Knox CA, Wagner-Mann CC. Postcardiotomy centrifugal assist: a single surgeon's experience. Artif Organs 2002;26:994-7

12. Jurmann MJ, Siniawski H, Erb M, Drews T, Hetzer R. Initial experience with miniature axial flow ventricular assist devices for postcardiotomy heart failure. Ann Thorac Surg 2004;77:1642-7 
VALORIZATION 
This thesis highlights the incidence, results, complication and innovative management of patients submitted to ECMO because of PC cardiogenic shock. This thesis provides several insights about the efficacy of ECMO in several settings of cardiac surgery or related interventions. Indeed, the favorable results obtained in complex settings, like HTx and general cardiac surgery confirm the efficacy of such a temporary support in case of refractory and life-threatening PC conditions, like graft rejection or cardiogenic shock due to variable reasons following cardiac surgery procedures. We could also demonstrate the impact of modified and more careful management (particularly in terms of anticoagulation and perioperative bleeding control) on the in-hospital results, with a positive trend reflecting on satisfactory ultimate outcome of treated patients.

The importance of the availability of the ECMO support, also as a back-up, has been clearly shown by our literature search in the setting of transcatheter valve implantation, particularly in the rescue and support of subjects experiencing severe complications, but also the impact of such a support in a prophylactic way, that is protecting hemodynamically the patients during high-risk procedure. The temporary support with ECMO enabled the performance of difficult and life-threatening procedure while guaranteeing cardio-circulatory and respiratory support during procedures which would have witness almost certainly complication or highly complex cardiocirculatory dysfunction and failure during or just after such procedures.

Further investigations about ECMO access, either in PC or non-PC settings, have showed that the access to implement such a support may also influence the patient outcome, particularly with regards to the complication types and rate with the peripheral approach demonstrated as less related to bleeding complications, although, the overall survival was not affected comparing the central or peripheral access.

The information available for PC-ECMO has been always rather poor and limited to single-centre experiences. A thorough review, with the provision on detailed information about prevalence, in relation to the overall cardiac surgery procedural number, conditions, patient profiles, support 
modalities, complications and outcome at short or long-term, have been also realized for adult and pediatric populations, and certainly precious for investigators and people involved in such settings. Finally, the presentation of an innovative management in the ECMO scenario, particularly helpful in patients experiencing either cardiac arrest or RV dysfunction before or after surgery, has been presented by means of a preliminary series of patients. This experience, as a multicenter investigation, showed the promising advantages of cannulating the pulmonary artery, also percutaneously. Such a technique will certainly be increasingly adopted in the ECMO setting, and particularly in PC subjects, allowing the management of complex situation that in the past were characterized by aggressive and often unsuccessful approaches. This new procedure and ECMO management will expand the options for complex patients, allowing the attending personnel to include minimally invasive and versatile configuration for PC-ECMO temporary support, thereby representing a major improvement in these patients. 
SUMMARY 
Chapter 2. In this chapter we present a single-centre experience of PC-ECMO after heart transplantation. The focus on survival after pre- and post-heart transplant mechanical circulatory support was described. Excellent results have been reported even in centers with low to moderate heart transplant volumes. In our experience, wise application of new mechanical circulatory support technologies, both pre- and post-transplant, can improve the results of heart transplantation.

Chapter 3. This study is a single-centre retrospective analysis of V-A ECMO support for 86 patients suffering PC cardiogenic shock after cardiac operations, excluding heart transplantation. Postoperative bleeding (overall rate $42 \%$ ) showed a trend towards a reduced rate over more recent years. The survival to discharge was $37 \%$, underlining the complexity of patients and the ECMO management.

Chapter 4. The performance of central versus peripheral cannulation in PC shock is a matter of debate. This meta-analysis, including 1.691 patients (980 patients underwent peripheral, and 711 central), is the only evidence-based analysis on this topic. The study showed comparable in-hospital survival rate between central and peripheral cannulation however, the risk of bleeding, continuous V-V hemofiltration and blood product transfusion, was significantly lower with the peripheral cannulation strategy.

Chapter 5. V-A ECMO support should be available at any centre performing TAVI and provides effective mechanical circulatory support if hemodynamic instability or cardio-circulatory injury/complication occurs. This review includes 102 patients (2\%) that required ECMO among 5.115 TAVI procedures (22 prophylactically, 66 as an emergency and 14 without a reported indication). The overall in-hospital survival was $73 \%$ (61\% in the emergency vs $100 \%$ in the prophylactic group) showing a good results of this treatment during complicated TAVI.

Chapter 6 and Chapter 7. State-of-art reviews on PC V-A ECMO after adult and pediatric cardiac operations. Indications, management of ECMO, and outcome are extensively reported providing a 
comprehensive information about all aspects for better understanding and further research on this setting.

Chapter 8. This chapter report an analysis of 15 patients affected by RV or BiV failure and supported with ECMO using an innovative management. Through the pulmonary artery cannulation, LV unloading in case of signs of blood stasis and pulmonary edema, or also providing isolated RV support as patients' outflow cannula, have been shown feasible and effective by means of the drainage or perfusion through such a cannula. Percutaneous implant has been also shown, making such a configuration attractive also for patients in whom a more aggressive approach might induce lifethreatening complications. Finally, the presence of a cannula in the pulmonary artery allows the performance of versatile ECMO management (so-called "Dynamic ECMO") changing the flow direction, drainage or perfusion, according to the patient needs and hemodynamic conditions. 
SAMENVATTING 
Hoofdstuk 2. In dit hoofdstuk presenteren we een single-center ervaring van PC-ECMO na harttransplantatie. De focus op overleving na pre- en post-hart transplantatie mechanische circulatieondersteuning werd beschreven. Uitstekende resultaten zijn gemeld, zelfs in centra met lage tot matige harttransplantatievolumes. Onze ervaring is dat verstandige toepassing van nieuwe mechanische ondersteuning van de bloedsomloop, zowel voor als na de transplantatie, de resultaten van harttransplantatie kan verbeteren.

Hoofdstuk 3. Deze studie is een single-center retrospectieve analyse van V-A ECMO-ondersteuning voor 86 patiënten die lijden aan PC cardiogene shock na hartoperaties, exclusief harttransplantatie. Postoperatieve bloedingen (totaalpercentage 42\%) vertoonden een trend in de richting van een verlaagd percentage over recentere jaren. De overleving na ontslag was 37\%, wat de complexiteit van patiënten en het ECMO-management onderstreept.

Hoofdstuk 4. De prestaties van centrale versus perifere canulatie bij pc-shock is een kwestie van debat. Deze meta-analyse, inclusief 1.691 patiënten (980 patiënten ondergingen perifere en 711 centrale), is de enige evidence-based analyse over dit onderwerp. De studie toonde een vergelijkbaar overlevingspercentage in het ziekenhuis aan tussen centrale en perifere canulatie, maar het risico op bloedingen, continue $\mathrm{V}-\mathrm{V}$ hemofiltratie en bloedproducttransfusie was significant lager met de strategie voor perifere canulatie.

Hoofdstuk 5. V-A ECMO-ondersteuning moet beschikbaar zijn in elk centrum dat TAVI uitvoert en biedt effectieve mechanische bloedsomloopondersteuning als hemodynamische instabiliteit of cardiovasculaire schade / complicatie optreedt. Deze beoordeling omvat 102 patiënten (2\%) die ECMO nodig hadden onder 5.115 TAVI-procedures (22 profylactisch, 66 als noodgeval en 14 zonder een gerapporteerde indicatie). De totale overleving in het ziekenhuis was $73 \%$ (61\% in de noodgeval 
versus $100 \%$ in de profylactische groep), wat een goede resultaten van deze behandeling tijdens gecompliceerde TAVI laat zien.

Hoofdstuk 6 en Hoofdstuk 7. Geavanceerde beoordelingen van PC V-A ECMO na cardiale operaties bij volwassenen en kinderen. Indicaties, beheer van ECMO en resultaten worden uitgebreid gerapporteerd met uitgebreide informatie over alle aspecten voor een beter begrip en verder onderzoek naar deze instelling.

Hoofdstuk 8. Dit hoofdstuk rapporteert een analyse van 15 patiënten die getroffen zijn door RV of BiV falen en ondersteund worden met ECMO met behulp van een innovatief management. Door middel van de canule van de longslagader is het lossen van LV in het geval van tekenen van bloedstasis en longoedeem, of ook het bieden van geïsoleerde RV-ondersteuning als de uitstroomcanule van de patiënt, haalbaar en effectief gebleken door middel van drainage of perfusie door een dergelijke canule. Percutaan implantaat is ook aangetoond, waardoor een dergelijke configuratie ook aantrekkelijk is voor patiënten bij wie een agressievere aanpak levensbedreigende complicaties kan veroorzaken. Ten slotte maakt de aanwezigheid van een canule in de longslagader het mogelijk om veelzijdig ECMO-beheer uit te voeren (zogenaamde "Dynamic ECMO") het veranderen van de stroomrichting, drainage of perfusie, afhankelijk van de behoeften van de patiënt en hemodynamische omstandigheden). 


\section{ACKHNOWLEDGEMENTS}

University of Maastricht Medical Center, leading institution in medicine, research and innovation.

Prof. Dr. R. Lorusso, promotor. Dear Professor Lorusso, I am very thankful for all the support received since our first meeting in 2016. Thank you for the patience and the right advice, it has been a privilege preparing my doctoral thesis under your supervision. Hope this could be the starting point of a successful collaboration.

Prof. Dr. J.G. Maessen, co-promotor. Dear Professor Maessen, I want to thank you for the attention you reserved to this project.

Prof. Dr. Bart Meyns and Prof. Dr. Udo Boeken, Members of the Doctoral Thesis Committee, thank you for your time in reviewing my thesis and for your valuable and constructive suggestions.

Dr. M. Pilato, maestro. Dear Dr. Pilato first I learned from you is that nothing is done beyond what is necessary for the patient's sake, and second the patient comes first of all especially before and after his operation. Dear Dr. Pilato, your model and the quality of your daily commitment is an essential and constant aspect of my professional activity: dedication, perseverance and sacrifice.

Dr. F. Clemenza and Dr. A. Arcadipane, co-authors. Dear Dr Clemenza and Dr Arcadipane, thank you for your continuous, continuous support and benevolence.

Dr. A. Luca, director of ISMETT. Dear Dr. Luca, thank you for all the continuous advices you gave me in order to improve myself. 
Prof. Dr. Bruno Gridelli, executive vice president, UPMC International; managing director, UPMC Italy; professor of surgery, UPMC; Prof. Dr. Piergiulio Conaldi, scientific director of ISMETT and Dr. Ugo Palazzo. Thank you for your time and consideration.

Prof. Dr. AF. Cereceda, professor, Karolinska University Hospital. Dear Professor Cereceda, I will always hold a very good memory of my fellowship at KI. I was able to deepen my knowledge in aortic surgery and research projects. ERC, as you did, will be my next.

Prof. R. Gaeta, Prof. Dr. C. Pellegrini, Dr. G. Zattera, Prof. Dr. Mario Viganò, cardiac surgeons. Dear Professor Gaeta, Pellegrini, Viganò and Dr. Zattera, I am extremely grateful for your teaching during my residency in cardiac surgery at University of Pavia. Dear Carlo, my big thank you today for the support during the difficult days of my job.

Dr. G. Tarelli, chief at Istituto Clinico Humanitas. Thank you for believing in me in a bad time of my professional lifetime.

Dr. P. Malvindi and Dr. M. Kowalewski, cardiac surgeons, co-authors, friends and paraninfo. Dear Pietro e Mariusz, I could not done it without you. I'm lucky to have met you during my career. Friendship and strategy will accompany us all the way.

Antonella and Francesca, wife and daughter, it is not easy be a wife and a daughter of a cardiac surgeon. The time we spend together will be never enough. Thank you for accepting my failings. Francesca, you slept since the first night and allowed to complete the thesis!

Mamma and Papà, I'd like to thank you for getting me where I am today. 
Elisabetta and Martino, nieces. I love you.

Annamaria, sister. I have been always proud of you.

Liedietta, Emanuela, Luigino, Nunzio, Antonella, Maria, Flavio, Gianluca, Sandra, Giovanni, Giuseppe, cousins. Thank you to be my family.

Zia Lidia, Zia Giovanna, Zia Anna and Zio Sergio, Giovanna, Pippi, Clelia and Mario. Thank you for your love.

Luca, Alberto, Salvatore, Gianluca, more than friends. Thank you.

Maria and Elena, friends. We stood.

Nonno Nunzio and Nonnaetta, Nonno Pinuccio and Nonna Anna, Zio Pippo and Zio Felice, Zio Gino and Zia Marialuisa and Franchino, thank you to drive me from the sky.

Giovanni, Giuseppe, Marta, Cristina, doctors at ISMETT. Friends, stay together.

Staff of Department for the Treatment and Study of Cardiothoracic Diseases and Cardiothoracic Transplantation and Department of Anesthesia and Intensive Care at ISMETT, dear Colleagues, Consultant, Fellow, thank you for your continuous help and your understanding. 


\section{ABOUT THE AUTHOR}

The author of this thesis, Giuseppe Maria Raffa, was born on May 6th in 1978 in Messina - Sicily, Italy. He graduated high school at Liceo Scientifico "Archimede" in Messina in 1997. During the same year he was admitted at the Medical school at the University of Messina. As visiting fellow, he has been involved in several exchange student programs during the university school (Neurosurgery Unit at University of Donetsk in Ukraine, General Surgery Unit at the American University of Beirut in Lebanon, ERASMUS exchange program at the Imperial College of London in England, and Pediatric Surgery Unit at University of Wroclaw in Poland) and in July 2003 he graduated Medical Doctor with a thesis on surgical treatment of congestive heart failure. In May 2003 he started his Internship as Medical Student at the Unit of Cardiac Surgery - University of Pavia and, after the completion of medical license examination, he was admitted to the Cardiac Surgery residency program (prof. Mario Viganò). Under the supervision of prof. R. Gaeta and prof. C. Pellegrini, he was involved in several research experiences and continued his surgical training in Pavia until August 2007. In September 2007 he moved to Stockholm for a Cardiothoracic Fellowship at Department of Cardiothoracic Surgery, Karolinska University Hospital of Stockholm under the supervision of prof. Anders-Franco Cereceda. During this year he had the opportunity to join a wide spectrum of aortic operations and to be involved in pre-clinical and clinical research projects on the "Relationship between endothelially derived vasoactive agents (endothelin-1 and endothelial nitric oxide) and diseases of the aortic valve and ascending aorta" and the "ASAP-project (Advanced Study of Aortic Pathology)". Alongside the clinical activity, he undertook study of end stage heart disease and completed his specialist degree thesis on domino heart transplantation. He graduated Specialist Cardiac Surgeon at University of Pavia in October 2008. Later on the same year he moved to Sudan as voluntary cardiac surgeon at The Salam Centre for Cardiac Surgery, Emergency NGO, in Khartoum and in 2009 he started his first job as specialist surgeon at Istituto Clinico Humanitas Milano, under the guide of dr. Tarelli, and with the collaboration of dr. P.G. Malvindi e dr. M. 
Kowalewski, he deepened his interest in left ventricle assist device, aortic surgery and minimally invasive procedures. In January 2013 he moved to Palermo where he currently lives and works as Attending in Cardiothoracic Surgery at The Institute of the Mediterranean for Transplantation and High Specialty Therapies (ISMETT) in collaboration with University of Pittsburgh Medical Centre and under the supervision of dr. Michele Pilato. Since September 1, 2016 Giuseppe Raffa is a Clinical Assistant Professor of Cardiothoracic Surgery, University of Pittsburgh. He is married to Antonella Iovino and has a daughter, Francesca (12 months). 


\section{LIST OF PUBBLICATIONS}

Pulmonary artery cannulation to enhance extracorporeal membrane oxygenation management in acute cardiac failure. Lorusso R, Raffa GM, Heuts S, Lo Coco V, Meani P, Natour E, Bidar E, Delnoij T, Loforte A. Interact Cardiovasc Thorac Surg. 2019 Oct 26. pii: ivz245. doi: 10.1093/icvts/ivz245.

Devil is in the detail-how to critically analyze studies designed to assess effectiveness of topical antibiotics in preventing sternal wound infections? Pasierski M, Zieliński K, Raffa GM, Lazar H, Lorusso R, Suwalski P, Kowalewski M. J Thorac Dis. 2019 Sep;11(Suppl 15):S1861-S1864. doi: 10.21037/jtd.2019.09.01.

Structured review of post-cardiotomy extracorporeal membrane oxygenation: part 1-Adult patients. Lorusso R, Raffa GM, Alenizy K, Sluijpers N, Makhoul M, Brodie D, McMullan M, Wang IW, Meani P, MacLaren G, Kowalewski M, Dalton H, Barbaro R, Hou X, Cavarocchi N, Chen YS, Thiagarajan R, Alexander P, Alsoufi B, Bermudez CA, Shah AS, Haft J, D'Alessandro DA, Boeken U, Whitman GJR. J Heart Lung Transplant. 2019 Nov;38(11):1125-1143. doi: 10.1016/j.healun.2019.08.014

Structured review of post-cardiotomy extracorporeal membrane oxygenation: Part 2-pediatric patients. Lorusso R, Raffa GM, Kowalewski M, Alenizy K, Sluijpers N, Makhoul M, Brodie D, McMullan M, Wang IW, Meani P, MacLaren G, Dalton H, Barbaro R, Hou X, Cavarocchi N, Chen YS, Thiagarajan R, Alexander P, Alsoufi B, Bermudez CA, Shah AS, Haft J, Oreto L, D'Alessandro DA, Boeken U, Whitman G. J Heart Lung Transplant. 2019 Nov;38(11):1144-1161. doi: 10.1016/j.healun.2019.07.004.

Limb ischemia in peripheral veno-arterial extracorporeal membrane oxygenation: a narrative review of incidence, prevention, monitoring, and treatment.Bonicolini E, Martucci G, Simons J, Raffa GM, Spina C, Coco VL, Arcadipane A, Pilato M, Lorusso R. Crit Care. 2019 Jul 30;23(1):266. doi: 10.1186/s13054-019-2541-3. Review.

Patterns of ascending aortic dilatation and predictors of surgical replacement of the aorta: A comparison of bicuspid and tricuspid aortic valve patients over eight years of follow-up. Agnese V, Pasta S, Michelena HI, Minà C, Romano GM, Carerj S, Zito C, Maalouf JF, Foley TA, Raffa G, Clemenza F, Pilato M, Bellavia D. J Mol Cell Cardiol. 2019 Jul 23;135:31-39. doi: 10.1016/j.yjmcc.2019.07.010.

Computational modeling of bicuspid aortopathy: Towards personalized risk strategies. Cosentino F, Scardulla F, D'Acquisto L, Agnese V, Gentile G, Raffa G, Bellavia D, Pilato M, Pasta S. J Mol Cell Cardiol. 2019 Apr 29. pii: S0022-2828(18)31034-4. doi: 10.1016/j.yjmcc.2019.04.026.

On the role of material properties in ascending thoracic aortic aneurysms. Cosentino F, Agnese V, Raffa GM, Gentile G, Bellavia D, Zingales M, Pilato M, Pasta S. Comput Biol Med. 2019 Apr 24;109:70-78. doi: 10.1016/j.compbiomed.2019.04.022

Identification of circumferential regional heterogeneity of ascending thoracic aneurysmal aorta by biaxial mechanical testing. Di Giuseppe M, Alotta G, Agnese V, Bellavia D, Raffa GM, Vetri V, Zingales M, Pasta S, Pilato M. J Mol Cell Cardiol. 2019 May;130:205-215. doi: 10.1016/j.yjmcc.2019.04.010. 
Sutured and sutureless repair of postinfarction left ventricular free-wall rupture: a systematic review. Matteucci M, Fina D, Jiritano F, Blankesteijn WM, Raffa GM, Kowaleski M, Beghi C, Lorusso R. Eur J Cardiothorac Surg. 2019 Apr 1. pii: ezz101. doi: 10.1093/ejcts/ezz101

Treatment strategies for post-infarction left ventricular free-wall rupture. Matteucci M, Fina D, Jiritano F, Meani P, Blankesteijn WM, Raffa GM, Kowaleski M, Heuts S, Beghi C, Maessen J, Lorusso R. Eur Heart J Acute Cardiovasc Care. 2019 Apr 1:2048872619840876. doi: $10.1177 / 2048872619840876$.

Long-term survival and major outcomes in post-cardiotomy extracorporeal membrane oxygenation for adult patients in cardiogenic shock. Meani P, Matteucci M, Jiritano F, Fina D, Panzeri F, Raffa GM, Kowalewski M, Morici N, Viola G, Sacco A, Oliva F, Alyousif A, Heuts S, Gilbers M, Schreurs R, Maessen J, Lorusso R. Ann Cardiothorac Surg. 2019 Jan;8(1):116-122. doi: 10.21037/acs.2018.12.04.

Hypertrophic Obstructive Cardiomyopathy and Subvalvular Mitral Apparatus Remodeling. Raffa GM, Pilato M. Ann Thorac Surg. 2019 Mar 2. pii:S0003-4975(19)30253-X. doi:10.1016/j.athoracsur.2019.01.056.

Transcathether aortic valve implantation with the new repositionable self-expandable Medtronic Evolut R vs. CoreValve system: evidence on the benefit of a meta-analytical approach. Kowalewski M, Gozdek M, Raffa GM, Słomka A, Zieliński K, Kubica J, Anisimowicz L, Kowalewski J, Landes U, Kornowski R, Lorusso R, Suwalski P. J Cardiovasc Med (Hagerstown). 2019 Apr;20(4):226-236.

[Surgical management of hypertrophic obstructive cardiomyopathy: the role of mitral valve]. G Ital Cardiol (Rome). Raffa GM, Romano G, Falletta C, Morsolini M, Turrisi M, Gentile G, Nugara C, Scarlata M, Clemenza F, Pilato M. 2019 Feb;20(2):109-116. doi: 10.1714/3093.30858.

Clinical complications during veno-arterial extracorporeal membrane oxigenation in post-cardiotomy and non post-cardiotomy shock: still the achille's heel. Lo Coco V, Lorusso R, Raffa GM, Malvindi PG, Pilato M, Martucci G, Arcadipane A, Zieliński K, Suwalski P, Kowalewski M. J Thorac Dis. 2018 Dec;10(12):6993-7004. doi: 10.21037/jtd.2018.11.103.

Neurological complications after cardiac surgery: a retrospective case-control study of risk factors and outcome. Raffa GM, Agnello F, Occhipinti G, Miraglia R, Lo Re V, Marrone G, Tuzzolino F, Arcadipane A, Pilato M, Luca A. J Cardiothorac Surg. 2019 Jan 25;14(1):23. doi: 10.1186/s13019019-0844-8.

The AVIATOR Registry: The right way to change perspective. Di Mauro M, Raffa GM, Foschi M, Calafiore AM. J Thorac Cardiovasc Surg. 2018 Dec 12. pii:S0022-5223(18)33269-0. doi: 10.1016/j.jtcvs.2018.11.116.

In-hospital outcomes after emergency or prophylactic veno-arterial extracorporeal membrane oxygenation during transcatheter aortic valve implantation: a comprehensive review of the literature. Raffa GM, Kowalewski M, Meani P, Follis F, Martucci G, Arcadipane A, Pilato M, Maessen J, Lorusso R; ECMO in TAVI Investigators Group (ETIG). Perfusion. 2019 Jan 11:267659118816555. doi:10.1177/0267659118816555.

Spontaneous coronary artery dissection: the great pretender. La Franca E, Falletta C, Raffa GM, Gentile G, Di Gesaro G, Baravoglia CMH, Gandolfo C, Frenda A, Caramanno G, Clemenza F, Pilato M. J Thorac Dis. 2018 Nov;10(Suppl 33):S4179-S4182. doi: 10.21037/jtd.2018.10.123. 
On the prospect of serum exosomal miRNA profiling and protein biomarkers for the diagnosis of ascending aortic dilatation in patients with bicuspid and tricuspid aortic valve. Gallo A, Agnese V, Coronnello C, Raffa GM, Bellavia D, Conaldi PG, Pilato M, Pasta S. Int J Cardiol. 2018 Oct 5. pii: S0167-5273(18)34637-0. doi: 10.1016/j.ijcard.2018.10.005.

Vancomycin paste in sternal wound infection prophylaxis-a genuine debate or futile attempts to justify flawed study? Kowalewski M, Raffa GM, Lorusso R, Anisimiowicz L, Lazar HL, Suwalski P. J Thorac Cardiovasc Surg. 2018 Sep;156(3):1128-1130. doi: 10.1016/j.jtcvs.2018.04.072.

Protracted aortic valve closure during peripheral veno-arterial extracorporeal life support: is intraaortic balloon pump an effective solution? Meani P, Delnoij T, Raffa GM, Morici N, Viola G, Sacco A, Oliva F, Heuts S, Sels JW, Driessen R, Roekaerts P, Gilbers M, Bidar E, Schreurs R, Natour E, Veenstra L, Kats S, Maessen J, Lorusso R. Perfusion. 2018 Jul 1:267659118787426. doi:10.1177/0267659118787426.

Meta-Analysis of Peripheral or Central ECMO in Postcardiotomy and Non-Postcardiotomy Shock. Raffa GM, Kowalewski M, Brodie D, Ogino M, Whitman G, Meani P, Pilato M, Arcadipane A, Delnoj T, Natour E, Gelsomino S, Maessen J, Lorusso R. Ann Thorac Surg. 2018 Jun 27. pii: S00034975(18)30882-8. doi: 10.1016/j.athoracsur.2018.05.063. Review.

Prognostic Value of the 6-Min Walk Test After Open-Heart Valve Surgery: EXPERIENCE OF A CARDIOVASCULAR REHABILITATION PROGRAM. Vitale G, Sarullo S, Vassallo L, Di Franco A, Mandalà G, Marazia S, Raffa GM, Giallauria F, Sarullo FM. J Cardiopulm Rehabil Prev. 2018 Sep;38(5):304-308. doi:10.1097/HCR.0000000000000340.

Pathoanatomic Findings and Treatment During Hypertrophic Obstructive Cardiomyopathy Surgery: The Role of Mitral Valve. Raffa GM, Romano G, Turrisi M, Morsolini M, Gentile G, Sciacca S, Armaro A, Stringi V, Mattiucci G, Magro S, Cosentino F, Clemenza F, Pilato M. Heart Lung Circ. 2018 Feb 14. pii: S1443-9506(18)30068-4. doi: 10.1016/j.hlc.2018.02.006

MiR34 inhibition induces human heart progenitor proliferation. Iannolo G, Sciuto MR, Raffa GM, Pilato M, Conaldi PG. Cell Death Dis. 2018 Mar 6;9(3):368. doi: 10.1038/s41419-018-0400-9.

Peripheral Artery Disease and Continuous Flow Left Ventricle Assist Device: An Engaging Complement Analysis May Help to Guide Treatment. Falletta C, Pasta S, Raffa GM, Crinò F, Sciacca S, Clemenza F. Artif Organs. 2018 Feb 13. doi: 10.1111/aor.13105

In-hospital outcome of post-cardiotomy extracorporeal life support in adult patients: the 2007-2017 Maastricht experience. Raffa GM, Gelsomino S, Sluijpers N, Meani P, Alenizy K, Natour E, Bidar E, Johnson DM, Makhoul M, Heuts S, Lozekoot P, Kats S, Schreurs R, Delnoij T, Montalti A, Sels JW, Poll MV, Roekaerts P, Maessen J, Lorusso R. Crit Care Resusc. 2017 Oct;19(Suppl 1):53-61.

Acute Obstructive Thrombosis of Sapien 3 Valve After Valve-in-Valve Transcatheter Aortic Valve Replacement for Degenerated Mosaic 21 Valve. Gandolfo C, Turrisi M, Follis F, Clemenza F, Falletta C, Gentile G, Liotta R, Raffa GM, Pilato M. JACC Cardiovasc Interv. 2017 Oct 12. pii: S19368798(17)31879-4.

Heart valve scaffold fabrication: Bioinspired control of macro-scale morphology, mechanics and micro-structure. D'Amore A, Luketich SK, Raffa GM, Olia S, Menallo G, Mazzola A, D'Accardi F, 
Grunberg T, Gu X, Pilato M, Kameneva MV, Badhwar V, Wagner WR. Biomaterials. 2017 Oct 6;150:25-37. doi: 10.1016/j.biomaterials.2017.10.011.

Comparative performance of transcatheter aortic valve-in-valve implantation versus conventional surgical redo aortic valve replacement in patients with degenerated aortic valve bioprostheses: systematic review and meta-analysis. Gozdek M, Raffa GM, Suwalski P, Kolodziejczak M, Anisimowicz L, Kubica J, Navarese EP, Kowalewski M; SIRIO-TAVI group. Eur J Cardiothorac Surg. 2017 Sep 8. doi: 10.1093/ejcts/ezx347

In Vivo Strain Analysis of Dilated Ascending Thoracic Aorta by ECG-Gated CT Angiographic Imaging. Pasta S, Agnese V, Di Giuseppe M, Gentile G, Raffa GM, Bellavia D, Pilato M. Ann Biomed Eng. 2017 Sep 7. doi: 10.1007/s10439-017-1915-4.

Training in Coronary Artery Bypass Surgery: Tips and Tricks of the Trade. Raffa GM, Malvindi PG, Kowalewski M, Sansone F, Menicanti L. Semin ThoracCardiovasc Surg. 2017 Summer;29(2):137142.

Particle image velocimetry study of the celiac trunk hemodynamic induced by continuous-flow left ventricular assist device. Scardulla F, Bellavia D, D'Acquisto L, Raffa GM, Pasta S. Med Eng Phys. 2017 Jul 12.

Meta-analysis to assess the effectiveness of topically used vancomycin in reducing sternal wound infections after cardiac surgery. Kowalewski M, Raffa GM, Szwed KA, Anisimowicz L. J Thorac Cardiovasc Surg. 2017 Jun 9

In Silico Shear and Intramural Stresses are Linked to Aortic Valve Morphology in Dilated Ascending Aorta. Pasta S, Gentile G, Raffa GM, Bellavia D, Chiarello G, Liotta R, Luca A, Scardulla C, Pilato M. Eur J Vasc Endovasc Surg. 2017 Jun 24.

Impact of Sternotomy and Pericardial Opening in Patients With Ventricular Septal Defects: Assess Before Sawing! Sanfilippo F, Di Gesaro G, Serretta R, Raffa G, Clemenza F. Anesth Analg. 2017 Jun 14.

Should subcutaneous implantable cardioverter-defibrillators be implanted in patients who are candidates for continuous flow left ventricular assist device? Raffa GM, Morsolini M, Gentile G, Coppola G, Sciacca S. Eur J Intern Med. 2017 May 12.

Critical care for migrants: Discussion on "Migrants crossing the Mediterranean Sea: an opportunity or a duty?" Roeggla G, Martucci G, Panarello G, Raffa GM. Intensive Care Med. 2017 Jul;43(7):1069-1070.

Modalities and Effects of Left Ventricle Unloading on Extracorporeal Life support: a Review of the Current Literature. Meani P, Gelsomino S, Natour E, Johnson DM, Rocca HB, Pappalardo F, Bidar E, Makhoul M, Raffa G, Heuts S, Lozekoot P, Kats S, Sluijpers N, Schreurs R, Delnoij T, Montalti A, Sels JW, van de Poll M, Roekaerts P, Poels T, Korver E, Babar Z, Maessen J, Lorusso R. Eur J Heart Fail. 2017 May;19 Suppl 2:84-91.

Clinical relevance of the International Society for Heart and Lung Transplantation consensus classification of primary graft dysfunction after heart transplantation: Epidemiology, risk factors, and outcomes. Sabatino Mario, Vitale Giuseppe, Manfredini Valentina, Masetti Marco, Borgese Laura, Raffa G, Loforte Antonio, Suarez Sofia Martin, Falletta Calogero, Marinelli Giuseppe, Clemenza 
Aortic surgery in Marfan patients with severe pectus excavatum. Raffa GM, Kowalewski M, Malvindi PG, Bertani A, Romano G, Sciacca S, Turrisi M, Armaro A, Stringi V, Montalbano G, Mattiucci G, Follis F, Pilato M. J Cardiovasc Med (Hagerstown). 2017 May;18(5):305-310.

Preserving the left subclavian artery patency in challenging proximal neck during thoracic endovascular aortic repair. Settepani F, Raffa GM, Malvindi PG, Tarelli G, Brambilla G, Pedicini V. J Cardiovasc Med (Hagerstown). 2017 May;18(5):374-377.

Migrants crossing the Mediterranean Sea: an opportunity or a duty? Raffa GM, Martucci G, Panarello G. Intensive Care Med. 2017 Mar 2. doi:10.1007/s00134-017-4726-Z.

Three-dimensional parametric modeling of bicuspid aortopathy and comparison with computational flow predictions. Pasta S, Gentile G, Raffa GM, Scardulla F, Bellavia D, Luca A, Pilato M, Scardulla C. Artif Organs. 2017 Feb 10. doi:10.1111/aor.12866.

Antihypertensive Treatments In Patients Affected By Aortic Valve Stenosis. Poggio P, Folesani G, Raffa GM, Songia P, Valenti V, Myasoedova V, Parolari A. Curr Pharm Des. 2016 Nov 23

Clinical Safety and Effectiveness of Endoaortic as Compared to Transthoracic Clamp for Small Thoracotomy Mitral Valve Surgery: Meta-Analysis of Observational Studies. Kowalewski M, Malvindi PG, Suwalski P, Raffa GM, Pawliszak W, Perlinski D, Kowalkowska ME, Kowalewski J, Carrel T, Anisimowicz L. Ann Thorac Surg. 2016 Oct 17. pii: S0003-4975(16)31140-7. doi: 10.1016/j.athoracsur.2016.08.072. Review.

Robotic mitral repair with sliding leaflet valvuloplasty and remodelling partial annuloplasty. Murashita T, Raffa GM, Wei LM, Badhwar V. Multimed Man Cardiothorac Surg. 2016 Sep 28;2016.

Computational fluid dynamics of the ascending aorta before the onset of type A aortic dissection. Malvindi PG, Pasta S, Raffa GM, Livesey S. Eur J Cardiothorac Surg. 2016 Sep 15.

From Small Coronary Artery Aneurysm to Giant Left Ventricle Aneurysm. Sciacca S., Raffa GM, Gentile G., Pilato M. MEDICAL PRINCIPLES AND PRACTICE, vol. 26, p. 87-89, ISSN: 10117571

Meta-analysis of uninterrupted as compared to interrupted oral anticoagulation with or without bridging in patients undergoing coronary angiography with or without percutaneous coronary intervention. Kowalewski M, Suwalski P, Raffa GM, Słomka A, Kowalkowska ME, Szwed KA, Borkowska A, Kowalewski J, Malvindi PG, Undas A, Windyga J, Pawliszak W, Anisimowicz L, Carrel T, Paparella D, Lip GY. Int J Cardiol. 2016 Aug 4;223:186-194.

Risk of stroke with "no-touch" - As compared to conventional off-pump coronary artery bypass grafting. An updated meta-analysis of observational studies. Kowalewski M, Suwalski P, Pawliszak W, Benetti F, Raffa GM, Malvindi PG, Carrel T, Paparella D, Anisimowicz L. Int J Cardiol. 2016 Aug 3;222:769-771.

Heart transplant program at IRCCS-ISMETT: impact of mechanical circulatory support on pre- and post -transplant survival. Raffa GM, Di Gesaro G, Sciacca S, Tuzzolino F, Turrisi M, Falletta C, Minà C, Romano G, Vitale G, Panarello G, Clemenza F, Pilato M. Int J Cardiol. 2016 Sep 15;219:358-61. 
Aortic surgery in Marfan patient with severe pectus excavatum. Raffa GM, Kowalewski M, Malvindi PG, Bertani A, Romano G, Sciacca S, Turrisi M, Armaro A, Stringi V, Montalbano G, Mattiucci G, Follis F, Pilato M. J Cardiovasc Med (Hagerstown). 2016 Jun 30.

PIWI-interacting RNA (piRNA) signatures in human cardiac progenitor cells. Vella S, Gallo A, Lo Nigro A, Galvagno D, Raffa GM, Pilato M, Conaldi PG. Int J Biochem Cell Biol. 2016 Jul;76:1-11.

International Participation in The STS Database Improves Outcomes: Initial Italian Experience. Raffa GM, Luca A, Badhwar V, Pilato M. Ann Thorac Surg. 2015 May;101(5):2028-9.

Cerebrovascular Events After No-Touch Off-Pump Coronary Artery Bypass Grafting, Conventional Side-Clamp Off-Pump Coronary Artery Bypass, and Proximal Anastomotic Devices: A MetaAnalysis. Pawliszak W, Kowalewski M, Raffa GM, Malvindi PG, Kowalkowska ME, Szwed KA, Borkowska A, Kowalewski J, Anisimowicz L. J Am Heart Assoc. 2016 Feb 18;5(2).

Can multiple previous treatment-requiring rejections affect biventricular myocardial function in heart transplant recipients? A two-dimensional speckle-tracking study. Romano G, Raffa GM, Licata P, Tuzzolino F, Baravoglia CH, Sciacca S, Scardulla C, Pilato M, Lancellotti P, Clemenza F, Bellavia D. Int J Cardiol. 2016 Feb 3;209:54-56.

Patients with bicuspid aortic valve are likely to receive an aortic valve prosthesis during prophylactic resection of their ascending aortic aneurysm. Raffa GM, Wu B, Pasta S, Morsolini M, Bellavia D, Romano G, Falletta C, Pietrosi A, Scardulla C, Pilato M. Int J Cardiol. 2016 Mar 1;206:97-100.

Early Distal Remodeling after Elephant Trunk Repair of Thoraco-Abdominal Aortic Aneurysms. Raffa GM, Pasta S, Gentile G, Scardulla F, Wu B, D’Ancona G, Follis F, Pilato M. J Biomech. 2015 Dec 31

Should pectus excavatum alter aortic root surgery in Marfan patients? A CT-scan guided surgical strategy through left anterior thoracotomy. Raffa GM, Gentile G, De Monte L, Pilato M. Seminars in Thoracic and Cardiovascular Surgery. Semin Thorac Cardiovasc Surg. 2015 Autumn;27(3):328-30.

Your Results, Explained: Clarity Provided by Row Percentages vs Column Percentages. Althouse A. Raffa GM, Kormos R. Ann Thorac Surg. 2016 Jan;101(1):15-7.

Constitutive modeling of ascending thoracic aortic aneurysms using microstructural parameters. Pasta S, Phillippi JA, Tsamis A, D'Amore A, Raffa GM, Pilato M, Scardulla C, Watkins SC, Wagner WR, Gleason TG, Vorp DA. Med Eng Phys. 2016 Feb;38(2):121-30.

Safety and efficacy of miniaturized extracorporeal circulation when compared with off-pump and conventional coronary artery bypass grafting: evidence synthesis from a comprehensive Bayesianframework network meta-analysis of 134 randomized controlled trials involving 22778 patients. Kowalewski M, Pawliszak W, Raffa GM, Malvindi PG, Kowalkowska ME, Zaborowska K, Kowalewski J, Tarelli G, Taggart DP, Anisimowicz L. Eur J Cardiothorac Surg. 2015 Nov 3

Stanford Type-A Acute Retrograde Aortic Dissections: from Surgical to Endovascular Strategy? Follis F, Raffa GM, D'Ancona G, Amaducci A, Follis F. Artif Organs. 2015 Oct 30.

An In Vitro Phantom Study on the Role of the Bird-Beak Configuration in Endograft Infolding in the Aortic Arch. Pasta S, Scardulla F, Rinaudo A, Raffa GM, D'Ancona G, Pilato M, Scardulla C. J Endovasc Ther. 2016 Feb;23(1):172-81. 
Off-Pump CABG Improves Short-Term Outcomes in High Risk Patients As Compared to On-Pump CABG: Meta-analysis. Kowalewski M, Pawliszak W, Malvindi PG; Bokszanski MP; Perlinski D; Raffa GM; Kowalkowska ME; Zaborowska K; Navarese EP; Kolodziejczak M; Kowalewski J; Tarelli G; Taggart DP; Anisimowicz L. J Thorac Cardiovasc Surg. 2016 Jan;151(1):60-77.e58.

Biomechanical implications of excessive endograft protrusion into the aortic arch after thoracic endovascular repair. Rinaudo A, Raffa GM, Scardulla F, Pilato M, Scardulla C, Pasta S. Comput Biol Med. 2015 Nov 1;66:235-41

How do I get my paper published? Insight from an EACTS luncheon. Raffa GM, Beyersdorf F. J Cardiovasc Med (Hagerstown). 2015 Aug 7.

Recurrent ventricular tachycardia in a patient with continuous flow left ventricle assist device: Successful management with radiofrequency ablation and medical treatment. Romano G, Raffa GM, Ruggieri A, Sgarito G, Falletta C, Sciacca S, Pilato M, Clemenza F. Int J Cardiol. 2015 Jul 1;190:198200.

Should Device Replacement Be the First Choice Strategy in Continuous-Flow Left Ventricle Assist Device Thrombosis? Analysis of 9 Events and Results after Endoventricular Thrombolysis. Raffa GM, D'Ancona G, Romano G, Falletta C, Sciacca S, Todaro C, Tuzzolino F, Pietrosi A, Amaducci A, Clemenza F, Scardulla C, Pilato M. Int J Cardiol. 2015 Jan 15;178:159-61.

Commentary: the bird-beak stent-graft configuration: the end of aortic arch endograft collapse? Pasta S, Raffa GM, D'Ancona G, Pilato M. J Endovasc Ther. 2014 Dec;21(6):803-4.

Thalassemia and Heart Surgery: A Case of Aortic Valve Repair after Endocarditis. Raffa GM, Mularoni A, Di Gesaro G, Vizzini G, Cipolla T, Pilato M. Ann Thorac Surgery. Ann Thorac Surg. 2015 Jan;99(1):e1-2.

Systemic or Endoventricular Thrombolysis to Treat HeartWare Left Ventricle Assist Device Thrombosis: A Clinical Dilemma. Raffa GM, D'Ancona G, Sciacca S, Pietrosi A, Hernandez Baravoglia CM, Turrisi M, Romano G, Armaro A, Stringi V, Clemenza F, Pilato M. Artif Organs. Artif Organs. 2015 Jun;39(6):526-9.

Chronic Stanford type A aortic dissection. Raffa GM, Pilato M, Armaro A, Ruperto C, Gentile G, Follis F. J Cardiovasc Med (Hagerstown). 2014 Aug 1.

Cusp repair during aortic valve-sparing operation: technical aspects and impact on results. Settepani F, Cappai A, Raffa GM, Basciu A, Barbone A, Berwick D, Citterio E, Ornaghi D, Tarelli G, Malvindi PG. J Cardiovasc Med (Hagerstown). 2015 Apr;16(4):310-7.2014 Jun 27.

Surgical treatment of systemic embolization by cardiac metastasis of lung cancer. Santise G, Raffa GM, Pilato M Asian Cardiovasc Thorac Ann. 2014 Nov;22(9):1103-5.

Preserving the left subclavian artery patency in challenging proximal neck during thoracic endovascular aortic repair. Settepani F, Raffa GM, Malvindi PG, Tarelli G, Brambilla G, Pedicini V. J Cardiovasc Med (Hagerstown). 2014 May 16. 
Vascular access complications in patients with continuous-flow left ventricle assist device undergoing percutaneous invasive procedures: A word of caution. Raffa GM, D'Ancona G, Sciacca S, Santise G, Pilato G, Turrisi M, Clemenza F, Pilato M. Int J Cardiol. 2014 Jul 1;174(3):768-9.

David operation: single center 10-year experience. Malvindi PG, Cappai A, Basciu A, Raffa GM, Barbone A, Citterio E, Ornaghi D, Tarelli G, Settepani F. J Cardiovasc Surg (Torino). 2015 Aug;56(4):639-45.

Analysis of postsurgical aortic false aneurysm in 27 patients. Malvindi PG, Cappai A, Raffa GM, Barbone A, Basciu A, Citterio E, Ornaghi D, Tarelli G, Settepani F. Tex Heart Inst J. 2013;40(3):27480 .

Minimally invasive direct coronary artery bypass in the era of percutaneous coronary intervention. Raffa GM, Malvindi PG, Ornaghi D, Citterio E, Cappai A, Basciu A, Barbone A, Fossati F, Tarelli G, Settepani F. J Cardiovasc Med (Hagerstown). 2015 Feb;16(2):118-24.

Hamartoma of mature cardiac myocytes: a cardiac tumour with preserved contractility. Raffa GM, Tarelli G, Balzarini L, Torta D, Monti L. Eur Heart J ardiovasc Imaging. 2013 Dec;14(12):1216.

Conversion to sternotomy during sternal-sparing coronary artery surgery. Raffa GM, Settepani F. J Card Surg. 2013 Jul;28(4):386-7.

Fish oil and postoperative atrial fibrillation: the Omega-3 Fatty Acids for Prevention of Post-operative Atrial Fibrillation (OPERA) randomized trial. Mozaffarian D, Marchioli R, Macchia A, Silletta MG, Ferrazzi P, Gardner TJ, Latini R, Libby P, Lombardi F, O'Gara PT, Page RL, Tavazzi L, Tognoni G; OPERA Investigators. JAMA. 2012 Nov 21;308(19):2001-11.

Sutureless repair for postinfarction cardiac rupture: A simple approach with a tissue-adhering patch. Raffa GM, Tarelli G, Patrini D, Settepani F. J Thorac Cardiovasc Surg. 2013 Feb;145(2):598-9.

Hamartoma of mature cardiac myocytes in adults and young: case report and literature review. Raffa GM, Malvindi PG, Settepani F, Melotti F, Monti F, Spaggiari P, Basciu A,Cappai A, Citterio E, Tarelli G. Int J Cardiol. 2013 Feb 20;163(2):e28-30.

Mitral and aortic valve prosthetic endocarditis following percutaneous closure of mitral paravalvular leak. Malvindi PG, Raffa GM, Cappai A, Barbone A, Basciu A, Settepani F, Citterio E, Ornaghi D, Tarelli G, Vitali E. Ann Thorac Surg. 2013 Feb;95(2):e45-6.

Left ventricular cleft. Cappai A, Malvindi PG, Raffa GM, Basciu A, Monti L. Eur Heart J Cardiovasc Imaging. 2013 Jan;14(1):14.

Left ventricular pseudoaneurysm following aortic valve prosthesis endocarditis. Malvindi PG, Ornaghi D, Tarelli G, Raffa GM. J Cardiovasc Med (Hagerstown). 2012 Jul;13(7):457-9.

Postsurgical aortic false aneurysm: pathogenesis, clinical presentation and surgical strategy. Raffa GM, Malvindi PG, Ornaghi D, Basciu A, Barbone A, Tarelli G, Settepani F. J Cardiovasc Med (Hagerstown). 2013 Aug;14(8):593-6.

6 Months of "Temporary" Support by Levitronix Left Ventricular Assist Device. Barbone A, Malvindi PG, Sorabella RA, Cortis G, Tosi PF, Basciu A, Ferrara P, Raffa G, Citterio E, Settepani F, Ornaghi D, Tarelli G, Vitali E. Artif Organs. 2012 Jul;36(7):639-42. 
Bicuspidy does not affect reoperation risk following aortic valve reimplantation. Malvindi PG, Raffa GM, Basciu A, Citterio E, Cappai A, Ornaghi D, Tarelli G, Settepani F. Interact Cardiovasc Thorac Surg. 2012 Jun;14(6):717-20.

Aortic valve replacement for paraprosthetic leak after transcatheter implantation. Raffa GM, Malvindi PG, Settepani F, Ornaghi D, Basciu A, Cappai A, Tarelli G. J Card Surg. 2012 Jan;27(1):47-51. Review article.

Long-term patency of saphenous vein patch plasty for left main coronary artery ostial disease. Raffa GM, Gaeta R, Carlo P, Marcello S, Viganò M. J Card Surg. 2011 Nov;26(6):629.

Giant left atrium syndrome. Raffa GM, Cappai A, Tarelli G. J Cardiovasc Med (Hagerstown). 2011 Oct;12(10):745-6.

Incidence of neoplastic disease following lung transplantation: a 17-year single centre experience. Pellegrini C, Nicolardi S, Savasta M, Totaro P, Meloni F, Ippoliti G, Oggionni T, Raffa G, D'Armini AM, Viganò M. Transplant Proc. 2011 May;43(4):1156-8.

Endothelin-1 and Brain Natriuretic Peptide plasma levels decrease after aortic surgery. Raffa GM, Jackson V, Liska J, Eriksson MJ, Caidahl K, Eriksson P, Cereceda AF. J Heart Valve Dis. 2010 Nov;19(6):724-30.

Domino heart transplantation: long-term outcome of recipients and their living donors: single center experience. Raffa GM, Pellegrini C, Viganó M. Transplant Proc. 2010;42(9):3688-93.

Aortic valve replacement by ministernotomy in redo patients with previous left internal mammary artery patent grafts. Gaeta R, Lentini S, Raffa G, Pellegrini C, Zattera G, Viganò M. Ann Thorac Cardiovasc Surg. 2010;16(3):181-6.

Treatment of ascending aorta disease with Bentall-De Bono operation using a mini-invasive approach. Perrotta S, Lentini S, Rinaldi M, D'armini AM, Tancredi F, Raffa G, Gaeta R, Viganó M. J Cardiovasc Med (Hagerstown). 2008;9(10):1016-22.

Minimally invasive video-assisted surgery for iatrogenic aortic root to right atrium fistula after incomplete percutaneous occlusion of patent foramen ovale: case report and review of the literature. Raffa GM, C. Pellegrini, S. Lentini, S. Perrotta, F. Tancredi, R. Gaeta, M. Viganò. J Cardiac Surgery. 2008;23:63-78.

Minimally invasive aortic valve replacement complicated by disseminate zygomycosis Raffa G, R. Gaeta, B. Castiglioni, B. Nocita, A. Viglio, M. Viganò. Bollettino della Società Medico Chirurgica di Pavia. 2005;118(2):183-90 . 
